\title{
Running axial mass of the nucleon as a phenomenological tool for calculating quasielastic neutrino-nucleus cross sections
}

\author{
Igor D. Kakorin ${ }^{1, \mathrm{a}}$, Konstantin S. Kuzmin ${ }^{1,2, \mathrm{~b}}$, Vadim A. Naumov ${ }^{1, \mathrm{c}}{ }_{(\mathbb{C}}$ \\ ${ }^{1}$ Bogoliubov Laboratory of Theoretical Physics, Joint Institute for Nuclear Research, 141980 Dubna, Russia \\ ${ }^{2}$ Institute for Theoretical and Experimental Physics, 117218 Moscow, Russia
}

Received: 22 June 2021 / Accepted: 13 December 2021 / Published online: 28 December 2021

(C) The Author(s) 2021

\begin{abstract}
We suggest an empirical rule-of-thumb for calculating the cross sections of charged-current quasielastic (CCQE) and CCQE-like interactions of neutrinos and antineutrinos with nuclei. The approach is based on the standard relativistic Fermi-gas model and on the notion of neutrino energy dependent axial-vector mass of the nucleon, governed by a couple of adjustable parameters, one of which is the conventional charged-current axial-vector mass. The inelastic background contributions and final-state interactions are therewith simulated using GENIE 3 neutrino event generator. An extensive comparison of our calculations with earlier and current accelerator CCQE and CCQE-like data for different nuclear targets shows good or at least qualitative overall agreement over a wide energy range. We also discuss some problematical issues common to several competing contemporary models of the CCQE (anti)neutrino-nucleus scattering and to the current neutrino interaction generators.
\end{abstract}

\section{Introduction}

An accurate calculation of the charged-current quasielastic (CCQE) neutrino-nucleus scattering cross sections remains an important issue to ensure the reliability and confidence level of extraction of neutrino oscillation parameters from atmospheric and accelerator neutrino experiments $[1,2]$. This problem is closely related to a large experimental uncertainty in the determination of the weak axial-vector and, to a lesser degree, pseudoscalar form factors of the nucleon and usually reduces to the experimental uncertainty in the nucleon axial mass, $M_{A}$, which governs the $Q^{2}$ evolution of the axial-

\footnotetext{
a e-mail: Kakorin@jinr.ru

be-mail: KKuzmin@theor.jinr.ru

c e-mail: VNaumov@ theor.jinr.ru (corresponding author)
}

vector form factor in the conventional dipole parametrization,

$F_{A}\left(Q^{2}\right)=F_{A}(0)\left(1+\frac{Q^{2}}{M_{A}^{2}}\right)^{-2}$,

where $Q^{2}$ is the modulus of the squared four-momentum transfer carried by the $W$-boson.

Efforts were made in recent years to extract the value of the parameter $M_{A}$ from $v_{\mu} \mathrm{D}, \bar{v}_{\mu} \mathrm{H}$, and $\pi^{ \pm}$electroproduction experiments [3-5], and from all available at that time data on $v / \bar{v}$ scattering processes off light, intermediate and heavy nuclei [6-8]. In the latter studies, the nuclear effects were accounted for by using the closure over the dinucleon states and one-pion exchange currents [9-11] for deuterium targets and by applying the standard Smith-Moniz relativistic Fermi-gas (SM RFG) model [12] (with the parameters extracted from electron-nucleus scattering measurements) for all other (heavier) nuclear targets. The most accurate models for the nucleon electromagnetic form factors were used in these calculations. It has been inferred from these studies that most of the then-existing CCQE and pion electroproduction data could be satisfactorily described with $M_{A}=M_{A}^{\mathrm{RFG}} \simeq 1 \mathrm{GeV}$ to within a few percent accuracy. This conclusion has been made before the modern high statistics measurements of the CCQE and CCQE-like scattering cross sections on carbon reach targets, performed in the FNAL experiments MiniBooNE [13,14], SciBooNE [15,16], MINERvA [17-19], and MINOS [20], and also in the $\mathrm{T} 2 \mathrm{~K}$ experiments with two near detectors - ND280 (off-axis) [21-25] and INGRID (on-axis) [26]. According to the RFG based calculations, the CCQE doubledifferential cross sections measured in MiniBooNE $[13,14]$ are well described with $M_{A}^{\mathrm{RFG}}=1.36 \pm 0.06 \mathrm{GeV}\left(v_{\mu}\right)$ and $1.31 \pm 0.03 \mathrm{GeV}\left(\bar{v}_{\mu}\right)$ (cf. also Refs. [27-32]). These values are in reasonable agreement with other recent low-energy data but are incompatible with the formal world-average value of $M_{A}^{\mathrm{RFG}}=1.026 \pm 0.021 \mathrm{GeV}$ [33] as well as with 


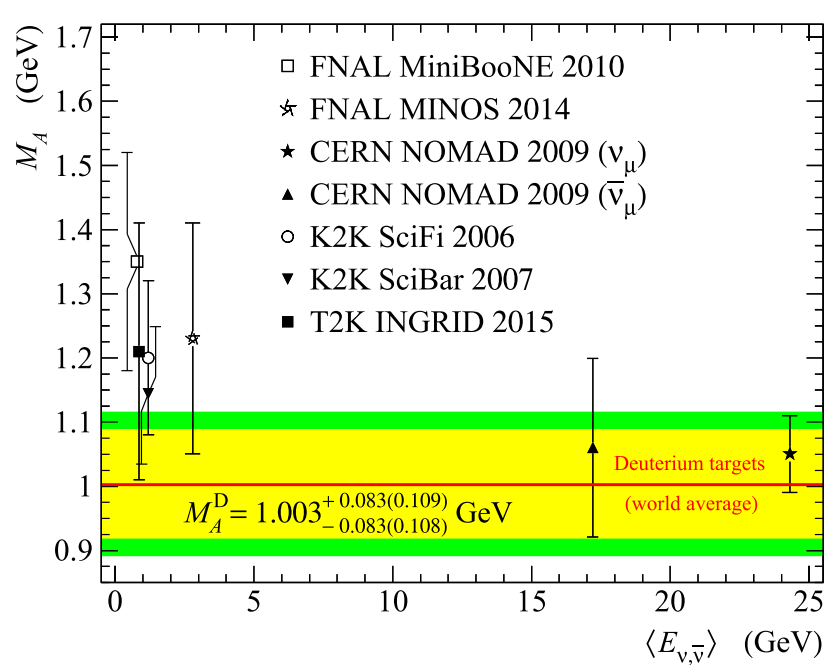

Fig. 1 The nucleon axial mass values vs. mean $v_{\mu} / \bar{v}_{\mu}$ energy, obtained in the experiments MiniBooNE [13], MINOS [20], NOMAD [34], SciFi [35], SciBar [36], and INGRID [26]. The straight line and surrounding shaded double band represent the $M_{A}$ value and its $1 \sigma$ and $2 \sigma$ uncertainties, as extracted from available deuterium data

the values of $1.07 \pm 0.11 \mathrm{GeV}\left(v_{\mu}\right)$ and $1.08 \pm 0.19 \mathrm{GeV}$ $\left(\bar{v}_{\mu}\right)$, extracted from the total CCQE cross sections measured at higher energies in the NOMAD experiment [34].

Figure 1 shows the values of the nucleon axial mass obtained in the experiments $[13,20,26,34-36]$ with composite (mainly carbon-rich) nuclear targets; the data are plotted as a function of the mean energy of the $v_{\mu} / \bar{v}_{\mu}$ beams. Also shown is the result of our statistical analysis to the "golden" (consistent, non-overlapping) $v_{\mu} \mathrm{D}$ and $\bar{v}_{\mu} \mathrm{H}$ data (see details below). It is amply clear that the values of $M_{A}^{\mathrm{RFG}}$ extracted using one or another version of the global RFG model from the low-energy data on heavy nuclear targets are in conflict with the deuterium data and also with the higher-energy data from NOMAD [34] and preceding experiments. Moreover, essentially all new low-energy data provide a hint that $M_{A}^{\mathrm{RFG}}$ increases with decreasing the mean $v_{\mu} / \bar{v}_{\mu}$ energy.

Modern explanations of the recent experiments include the effects beyond the scope of the naive RFG and impulse approximation. Among these are various extensions of the standard (global) RFG model, such as local Fermi gas (LFG) model [37], local density approximation (LDA) [38], and spectral function (SF) approach [39-48]; relativistic mean field and relativistic Green's function models [49,50]; charged meson-exchange currents (MEC), intermediate $\Delta$ isobar or multi-nucleon excitations [51,52], short-range and long-range correlations (SRC and LRC) within random phase approximation (RPA) [53-55]; quantum-kinetic transport equations (implemented in the GIBUU code) [56, 57]; parametrization of the observed enhancement in the transverse electron quasielastic response function (presumably because of MEC) [45,58-60]; a variety of so-called superscaling models, e.g., SuSA [61,62], SuSAv2 [63,64], SuSAv2-MEC [65-67], and SuSAM* [68]. The most comprehensive microscopic and phenomenological models usually increase the CCQE cross sections at low energies, thus providing better data explanation without increasing $M_{A}^{\mathrm{RFG}}$ (see Refs. [69-74] for reviews and further references).

The main purposes of this study are to clarify the experiential state-of-the-art with the nucleon axial mass and provide a simple phenomenological method for an accurate description of the CCQE $v$ and $\bar{v}$ interactions with nuclei at energies of interest for neutrino oscillation experiments, within the frameworks of conventional RFG model, but at the expense of having two adjustable parameters (instead of the only one, $M_{A}$ ) in the nuclear axial-vector structure function. The suggested recipe should never be considered as an alternative or competitor to the detailed microscopic models. Rather, it can serve as a complementary empirical tool which can easily be implemented in any Monte Carlo neutrino event generator ${ }^{1}$ and used in the analyses of the experiments with accelerator and atmospheric neutrino and antineutrino fluxes.

\section{Running axial mass}

The idea of the prescribed method is to calculate the cross sections for the CCQE $v / \bar{v}$ interactions with nuclei other than hydrogen and deuterium by using the neutrino energy dependent running axial-vector mass, $M_{A}^{\text {run }}$, in the charged weak hadronic current, instead of the conventional constant axialvector (dipole) mass $M_{A}$; below the latter will be referred to as the current axial mass.

Some motivation is required for the suggested "trick" in which the axial form factor $F_{A}$ - the function of $Q^{2}$ only - is replaced by a function dependent on $Q^{2}$ and neutrino energy $E_{v}$ (through the function $M_{A}^{\text {run }}\left(E_{v}\right)$ ). According to the above-listed microscopic models, the multi-nucleon excitation mechanisms (such as the RPA long-range correlations or two particle - two hole $(2 p 2 h)$ channels contributions caused by meson exchange currents) lead to an enhancement of the neutrino-nucleus flux-folded cross sections at low neutrino energies (see, e.g., Ref. [54]). The experimental hints shown in Fig. 1 suggest that this enhancement can be phenomenologically reproduced only by adjusting the nucleon axial mass parameter, $M_{A}$, and remaining within the framework of the simple RFG approach that is without accounting for the nontrivial nuclear effects. The most straightforward way to do this would be to construct a suitable function $M_{A}^{\text {run }}\left(Q^{2}\right)$ and fine-tune it on the appropriate datasets.

A more simple empirical solution is to use the well-known (close to linear) correlation between the mean $Q^{2}$ value,

\footnotetext{
1 The model is implemented into the GENIE neutrino generator (version 2.11.0 and higher) as an option.
} 
$\left\langle Q^{2}\right\rangle$, and neutrino energy, $E_{v}$ (see, e.g., Refs. [75-80]), which allows a two-step approximation in the calculating the flux-folded cross sections: considering that $M_{A}^{\text {run }}\left(Q^{2}\right)$ is a relatively weakly dependent function of $Q^{2}$, one can approximately replace variable $Q^{2}$ in this function with its mean value, $\left\langle Q^{2}\right\rangle$, and then use the mentioned correlation between $\left\langle Q^{2}\right\rangle$ and $E_{v}$,

$M_{A}^{\text {run }}\left(Q^{2}\right) \longmapsto M_{A}^{\text {run }}\left(\left\langle Q^{2}\right\rangle\right) \longmapsto M_{A}^{\text {run }}\left(E_{\nu}\right)$,

thus arriving at the notion of running axial mass of the nucleon, $M_{A}^{\mathrm{run}}\left(E_{\nu}\right)$. The energy dependence of $M_{A}^{\mathrm{run}}$ can be then retrieved from available CCQE data. The outlined customization of the hadronic current must be treated as a purely empirical prescription to account for the experimental evidence of nuclear effects beyond the RFG model.

The function $M_{A}^{\text {run }}$ must be parametrized in such a way that it asymptotically approaches the current (constant) $M_{A}$ at high energies and describes the lower-energy data and in general, it will be different for different modifications of the RFG models and other inputs. In the present analysis, we adopt the very simple parametrization,

$M_{A}^{\mathrm{run}}=M_{0}\left(1+\frac{E_{0}}{E_{v}}\right)$,

in which $E_{v}$ is the lab-frame neutrino energy which can be treated as Lorentz invariant $\left(E_{v}=\left(s-M^{2}\right) / 2 M\right.$, where $M$ is the mass of the target nucleus) and the constant parameters of $M_{0}$ and $E_{0}$ are obtained from the global fit to available accelerator data on the CCQE $v_{\mu}$ and $\bar{v}_{\mu}$ interactions with nuclei. Hence the modified hadronic current formally retains its Lorentz-transformation property albeit loses the fundamental meaning. It turns out that the parametrization (1) is universal in the sense that it works rather well for all mediumto-heavy nuclear targets and at all available $v / \bar{v}$ energies.

In our fit to $M_{A}^{\text {run }}$, the $v_{\mu} \mathrm{D}$ and $\bar{v}_{\mu} \mathrm{H}$ cross sections are exploited for adjusting the parameter $M_{0}$ only, inasmuch as the function $M_{A}^{\text {run }}\left(E_{v}\right)$ can be applied to the $v / \bar{v}$ CCQE scattering from the heavier nuclear targets. Since $M_{A}^{\text {run }} \rightarrow M_{0}$ at high energies, $E_{v} \gg E_{0}$, where the RFG model works rather well, the constant $M_{0}$ can be treated as the current axial-vector mass $M_{A}$. On the other hand, the value of $M_{A}$ can be independently extracted from a fit to the $v_{\mu} \mathrm{D}$ data for which the nontrivial (beyond RFG) nuclear effects are relatively small and better understood. Such approach will provide the predictive power of the formalism over a rather wide kinematic region.

\subsection{Parameters of the RFG model}

The nuclear Fermi momenta, $p_{F}$, and binding energies (usually identified with the separation energies), $E_{b}$, are rather uncertain and values of these parameters used in the literature vary greatly (see Refs. $[84,85]$ for a more sophisticated treatment of these matters). Both parameters are usually subject of adjustment in each specific experiment. For a certain unification, in the ensuing calculations we will use the following interpolation formulas:

$$
\begin{aligned}
& p_{F}=p_{F}^{0}\left[1-\frac{4.2}{A}+\left(\frac{6.0}{A}\right)^{2}-\left(\frac{5.3}{A}\right)^{3}\right], \\
& E_{b}=E_{b}^{0}\left[1-\frac{2.26}{\xi}+\left(\frac{1.73}{\xi}\right)^{2}-\left(\frac{1.21}{\xi}\right)^{3}\right],
\end{aligned}
$$

where $p_{F}^{0}=270 \mathrm{MeV}, E_{b}^{0}=50.4 \mathrm{MeV}, \xi=Z / A^{1 / 3}, Z$ is the atomic number, and $A$ is the mass number. These interpolations are obtained from the available data on electronnucleus scattering $[82,83]$ and are sufficiently accurate for all nuclei with $A \geq 6$, see Fig. 2. The interpolation (2) for the Fermi momenta is numerically close to that suggested in Ref. [86] and to the default GENIE inputs. Note that the previously published data [81] shown in Fig. 2 were partially updated in Ref. [82]. The default GENIE values of the binding energies for $\xi \sim 5-6$ are based on the obsolete data of Ref. [81] leading to a certain "dip" in the function $E_{b}(\xi)$, which however does not affect the following analysis.

The proton and neutron Fermi momenta are calculated in the conventional way $[81,86]$ as, respectively,

$p_{F}^{p}=\left(\frac{2 Z}{A}\right)^{1 / 3} p_{F}$ and $p_{F}^{n}=\left(\frac{2 N}{A}\right)^{1 / 3} p_{F}$,

where $N=A-Z$. These relations are based on the simplest assumption that the density of nuclear matter is approximately constant irrespective of the proton-to-neutron ratio $Z / N$.

\subsection{CCQE-like background due to pion production}

For calculations of the inelastic contributions to the CCQElike background with the $\mathrm{SMRFG}+M_{A}^{\text {run }}$ model, we made several modification from the standard GENIE 3 configuration [87]. Specifically, for simulation of the single-pion neutrinoproduction (an essential contribution to the FSI correction for the CCQE-like cross sections, see Sect. 4.1) we use the modified extended Rein-Sehgal model (ExRS or KLN) [88] supplemented by the pion-pole contribution to the hadronic axial current derived in Ref. [89]. This model is referred to as Berger-Sehgal (BS) or KLN-BS model in GENIE 3. Pauli-blocking effect is taken into account for the nuclear targets. Next, we refused the renormalization of the Breit-Wigner factors suggested in the original Rein-Sehgal model [90] and used in GENIE 3 in a slightly modified form. Among several physical and technical reasons of that refusal, we only mention here that the normalization integral for the $S$-wave resonances diverges, leading to an unreasonable ambiguity due to an unphysical cutoff in invariant hadronic 


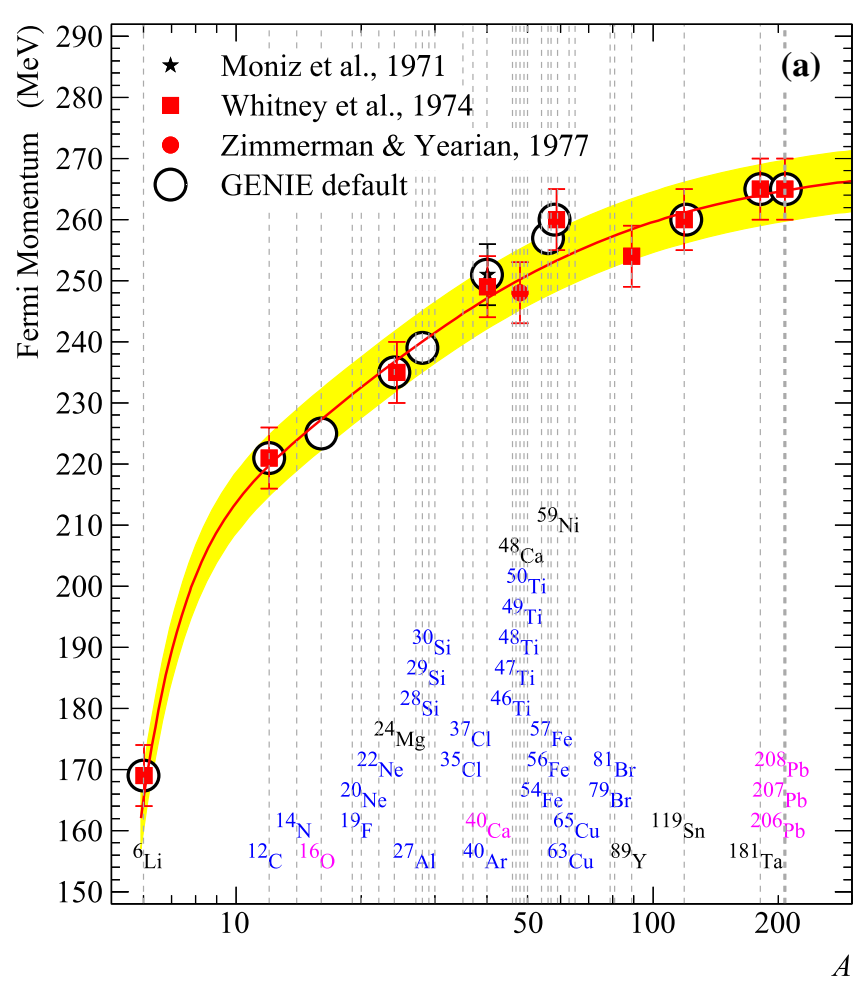

Fig. 2 The parametrizations of the Fermi momentum vs. mass number (a) and separation energy vs. parameter $\xi=Z / A^{1 / 3}$ (b) according to Eqs. (2). The data points are taken from Refs. [81-83]. The filled bands indicate the $1 \sigma$ uncertainties of the interpolations. The dashed vertical lines represent the values of $A$ and $\xi$ for the isotopes studied

mass [91]. The KLN-BS model properly takes into account the interference between the amplitudes of the resonances which have the same spin and orbital angular momentum. However, the current GENIE release of the KLN-BS model neglects this effect and operates with an incoherent sum over 17 resonance families. The interference essentially affects both on the absolute values and on the shapes of the pion production cross sections, but integrally causes less than $\sim 2 \%$ effect in the CCQE-like cross section calculations. This is within the level of expected accuracy of the GENIE3 simulation procedure (which employs somewhat rough simplifications) and is well within the systematic errors of the experimental data under subsequent consideration. All the mentioned features and flaws are subject of further improvements of the GENIE package.

Instead of the GENIE default "resonance" axial-vector mass value $M_{A}^{\mathrm{RES}}=1.12 \mathrm{GeV}[7,87]$, in the present analysis we use the updated value of $1.18 \mathrm{GeV}$, obtained from the new global fit to all available $v_{\mu} \mathrm{D}$ single-pion production data [91], for which the nonresonant background (NRB) is small compared to the resonance contribution. Note that treatment of NRB in GENIE is also different from that in the KLN-BS model. All other KLN-BS model inputs (resonance masses,

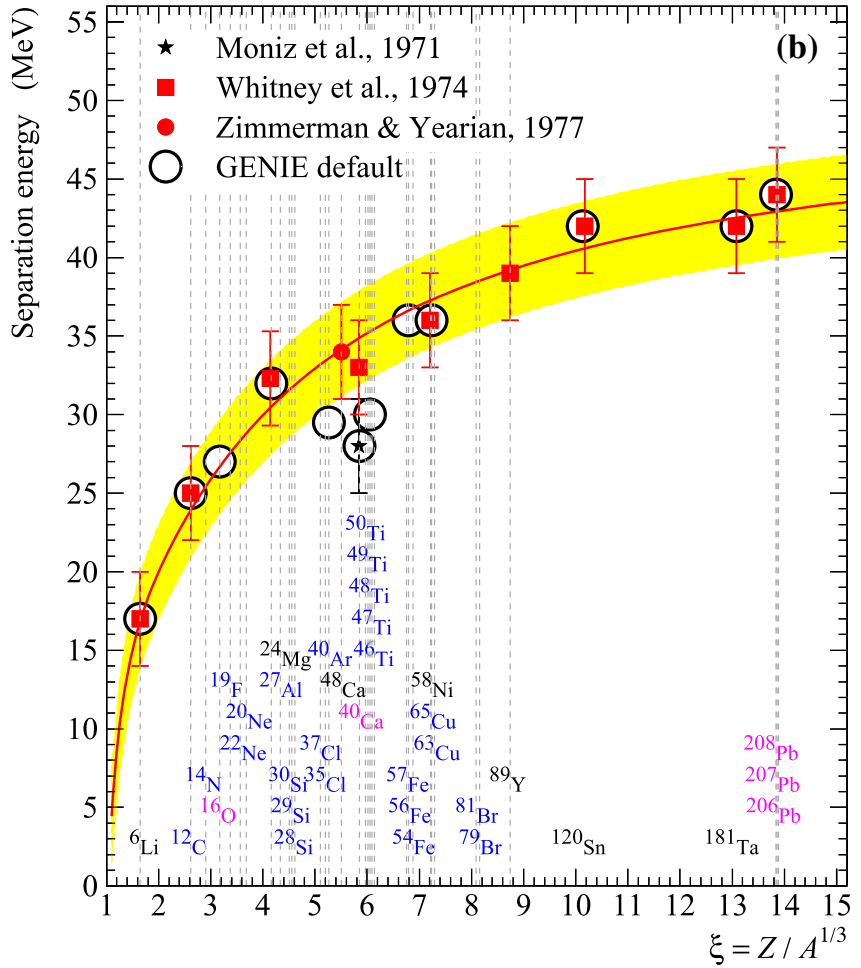

in the experiments [81-83] and for those noticeably contained in the detector materials employed in the neutrino experiments whose data are involved into the present analysis. Current GENIE 3 default inputs are also indicated

widths, decay mode fractions) are updated according to the most recent data, as suggested in Ref. [92].

The parametrization of the vector CCQE form factors from the GENIE default "BBA(05)" model are replaced to a more accurate "BBBA $25(07)$ " one $[4,5]$. In all our calculations performed with the models/tunes incorporated in GENIE, we thoroughly accounted for all essential experimental features (cuts, elemental compositions of the detectors, etc.). The results of all other (non-GENIE) models under consideration are reproduced exactly as provided by their authors.

\section{Statistical analysis}

The measurements of the CCQE $v_{\mu} / \bar{v}_{\mu}$ cross sections were carried out from the mid-60s to present day, in the experiments at ANL [93-99], BNL [79,100-105], FNAL [13-19, 106-118] LANL (LSND) [119], CERN [34,76,120-135], IHEP [80,136-142], K2K [35], and T2K [21-24,26,143]. The detector targets employed in these experiments were hydrogen [79], deuterium [94-101,104-106,134], water $[35,143,144]$ mineral oil $[13,14,17,18]$, aluminium [136139], argon [135], steel [93], iron [110], freon [76,80, 
120-124, 127-130,140-142], propane-freon mixtures [130133], neon-hydrogen mixture [107-109], complex carbonbearing media (hydrocarbon, propane, polystyrene, etc.) [13$18,34,102,103,111-119,125,126]$, and complex carbonaceous targets of the T2K near detectors [21-24,26]. Additional information can be found in review articles and data compilations [145-155] for the earlier and [73,74] for the contemporary experiments.

For our statistical analysis, we employ the CCQE data on the total, flux-averaged single-, and double-differential cross sections, as well as the flux-weighted $Q^{2}$ distributions, $\left\langle d N / d Q^{2}\right\rangle$, or $Q^{2}$ distributions specified by the mean $\nu_{\mu} / \bar{\nu}_{\mu}$ energy. The full data set is formed by the most statistically reliable and self-contained measurements which were not reexamined (as a result of enlarged statistics, revised data processing, and so on) in the later reports of the same experimental groups. We avoid using the data from the experiments with poorly known (anti)neutrino energy spectra and/or with non-active detector targets (see Ref. [8] for the details of the selection criteria). Namely, we use the results of the following experiments operating with different nuclear targets:

- hydrogen: BNL 1980 [79] $\left(d N_{\bar{\nu}} / d Q^{2}, 5\right)$;

- deuterium: ANL 1977 [97] $\left(\sigma_{v}, 8\right)$, ANL 1982 [99] $\left(\left\langle d N_{v} / d Q^{2}\right\rangle, 39\right)$, BNL $1990[104,105]\left(\left\langle d N_{v} / d Q^{2}\right\rangle\right.$, 37), FNAL 1983 [106] ( $\left.\left\langle d N_{v} / d Q^{2}\right\rangle, 20\right)$, and Big European Bubble Chamber (BEBC) at CERN 1990 [134] $\left(\left\langle d \sigma_{v} / d Q^{2}\right\rangle, 8\right)$;

- $\mathrm{Ne}-\mathrm{H}_{2}$ mixture: FNAL 1984 [107] $\left(d N_{\bar{v}} / d Q^{2}, 14\right)$;

- aluminium: IHEP-ITEP $1985[138,139]\left(\sigma_{\nu}, \sigma_{\bar{v}}\right.$, and $\left\langle d \sigma_{v+\bar{v}} / d Q^{2}\right\rangle, 8$ in each dataset);

- carbon-rich media: CERN NOMAD 2009 [34] $\left(\sigma_{\nu}, \sigma_{\bar{\nu}}\right.$, 10 and 6, respectively), FNAL MiniBooNE 2010 [13] $\left(\left\langle d^{2} \sigma_{\nu} / d E_{\mu} d \cos \theta_{\mu}\right\rangle, 137\right)$, and MiniBooNE 2013 [14] $\left(\left\langle d^{2} \sigma_{\bar{v}} / d E_{\mu} d \cos \theta_{\mu}\right\rangle, 78\right)$, T2K INGRID 2015 [26] $\left(\sigma_{v}\right.$, $2)$, and T2K ND280 2014 [23] $\left(\sigma_{\nu}, 5\right)$.

- liquid-argon time projection chamber (LAr-TPC) 2007 [135] $\left(\sigma_{v}, 1\right)$;

- freon $\left(\mathrm{CF}_{3} \mathrm{Br}\right)$, propane $\left(\mathrm{C}_{3} \mathrm{H}_{8}\right)$, and freon-propane compounds: bubble chamber Gargamelle (GGM) at CERN 1979 [132,133] $\left(\left\langle d N_{\bar{v}} / d Q^{2}\right\rangle, 13\right)$; IHEP babble chamber SKAT 1990 [142] $\left(\left\langle d \sigma_{\nu} / d Q^{2}\right\rangle,\left\langle d \sigma_{\bar{v}} / d Q^{2}\right\rangle, 8\right.$ and 7, respectively).

In the brackets we show the data types and numbers of the experimental bins involved into the analysis. Hence the full data set for the our analysis consists of 422 data points with 290,124 , and 8 ones for, respectively, $v_{\mu}(68.7 \%$ of the full data set), $\bar{v}_{\mu}(29.3 \%)$, and cumulative $v_{\mu}+\bar{v}_{\mu}(1.9 \%)$ cross sections and distributions. The data are presented as 215, 31, 128 , and 48 experimental data points for, respectively, the flux-folded double-differential CCQE cross sections measured by MiniBooNE (51\% of the full data set), differen- tial in $Q^{2}$ cross sections (7.4\%), unnormalized $Q^{2}$ distributions $(30.3 \%)$, and flux-unfolded total CCQE cross sections $(11.4 \%)$. The full data set covers a wide energy range - from the CCQE reaction threshold to about $100 \mathrm{GeV}$. The data subset used for extracting the current axial mass $M_{A}\left(\equiv M_{A}^{\mathrm{D}}\right)$ contains 117 data points (that constitutes $27.7 \%$ of the full data set) and is composed of the results of the experiments ANL 1977 [97], 1982 [99], BNL 1980 [79], 1990 [104, 105], FNAL 1983 [106], and CERN BEBC 1990 [134].

In the present analysis, we do not utilize the most recent data from T2K [24,25, 143, 144, 156-160], and MINERvA [17-19,111-116,161], as well as the CCQE-like doubledifferential cross sections and both CCQE and CCQE-like single-differential and total cross sections from MiniBooNE $[13,14]$. Instead, a limited but representative part of these data is used for an attentive and thorough verification of the $\mathrm{SMRFG}+M_{A}^{\text {run }}$ model and for a comparative analysis of several competing models (see Sect. 5). We plan to study the remaining and new data in a future dedicated work.

We use the ordinary least-square statistical model:

$\chi^{2}=\sum_{i}\left\{\sum_{j \in G_{i}} \frac{\left[N_{i} T_{i j}(\lambda)-E_{i j}\right]^{2}}{\sigma_{i j}^{2}}+\frac{\left(N_{i}-1\right)^{2}}{\delta_{i}^{2}}\right\}$.

Here, the index $i$ labels the experiments or data groups $G_{i}$, index $j \in G_{i}$ enumerates the bin-averaged experimental data $E_{i j}$ from the group $G_{i}, \sigma_{i j}$ is the error of $E_{i j}$, without normalization uncertainties (due to the $v / \bar{v}$ flux indetermination and other sources). The normalization factors, $N_{i}$ (individual for each data group $G_{i}$ ), are treated as fitting parameters and are included into the ordinary penalty term, $\left(N_{i}-1\right)^{2} / \delta_{i}^{2}$, where $\delta_{i}$ is the relative normalization error. The value $T_{i j}(\lambda)$ represents the associated (also binaveraged) model prediction, which is a function of a set of fitting parameters $\lambda=\left(\lambda_{1}, \lambda_{2}, \ldots\right)$; in our particular case, $\lambda=\left(M_{0}\right)$ for hydrogen and deuterium targets and $\lambda=\left(M_{0}, E_{0}\right)$ for all other nuclear targets.

The minimization procedure is significantly simplified by substituting into Eq. (3) $N_{i}=\mathscr{N}_{i}(\boldsymbol{\lambda})$, where the numbers

$\mathscr{N}_{i}(\lambda)=\frac{1+\delta_{i}^{2} \sum_{j \in G_{i}} \sigma_{i j}^{-2} T_{i j}(\lambda) E_{i j}}{1+\delta_{i}^{2} \sum_{j \in G_{i}} \sigma_{i j}^{-2} T_{i j}^{2}(\lambda)}$

are the solutions of the minimization equations $\partial \chi^{2} / \partial N_{i}=0$ (see Appendix A for a slightly more complicated case). The $\chi^{2}$ value for the final fit to all data includes the penalty term $\left[\left(M_{0}-M_{A}^{\mathrm{D}}\right) / \Delta M_{A}^{\mathrm{D}}\right]^{2}$,

which provides a "soft anchoring" of the parameter $M_{0}$ to the current axial mass $M_{A}^{\mathrm{D}} \pm \Delta M_{A}^{\mathrm{D}}$ obtained from the fitting of the robust deuterium data only.

For extracting the value of $M_{A}$ from the CCQE $v_{\mu} \mathrm{D}$ data, the authors of the experiments (see, e.g., Refs. [101,104, 


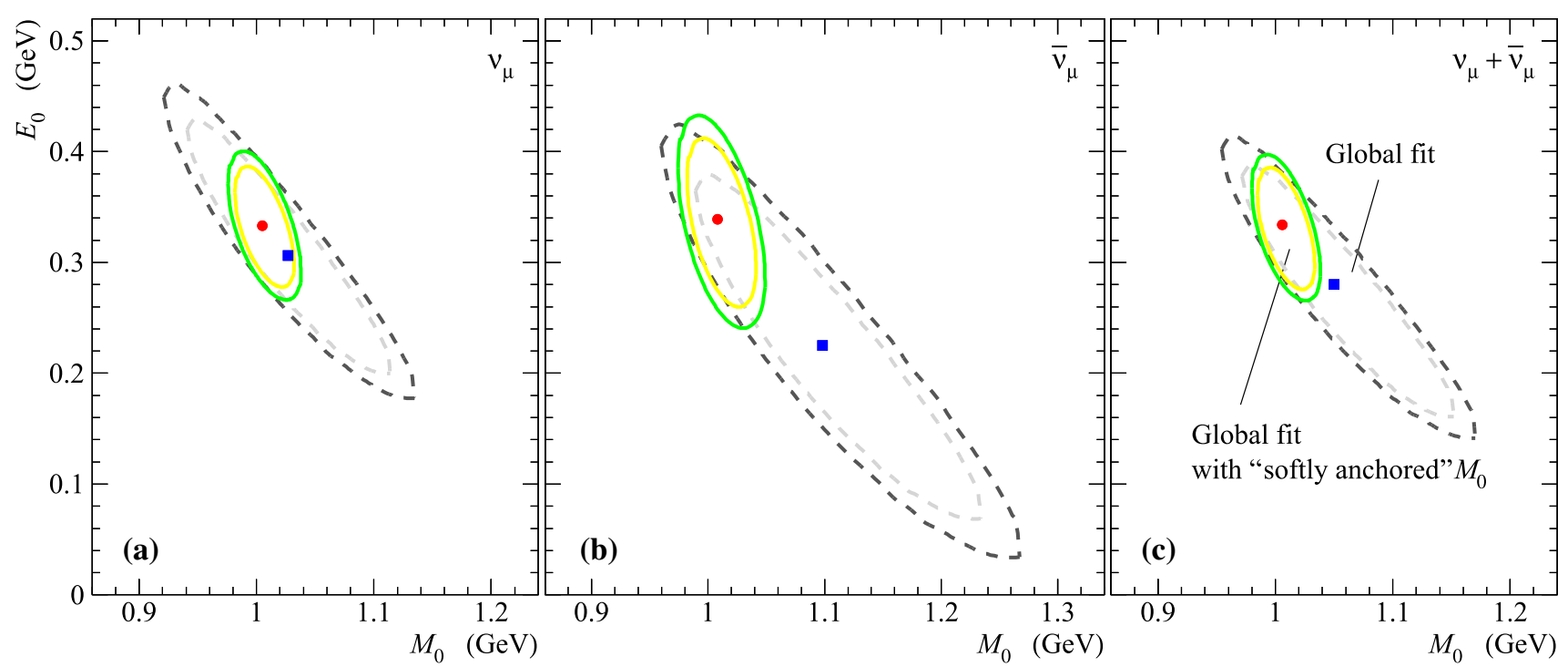

Fig. 3 Marginalized confidence contours in the $\left(M_{0}, E_{0}\right)$ plane obtained in the global fits for $v_{\mu}(\mathbf{a}), \bar{v}_{\mu}(\mathbf{b})$, and $v_{\mu}+\bar{v}_{\mu}$ (c) CCQE datasets. The smaller solid contours are obtained from the fits in which

106]) usually take into account the Pauli exclusion principle. In our analysis we try to use whenever possible the raw, uncorrected $v_{\mu} \mathrm{D}$ data (mainly $Q^{2}$ distributions). To account for the nuclear effects besides the trivial effects of Fermi motion and deuteron binding we adopt the closure approximation over the dinucleon states following Ref. [11], where the MEC contributions were estimated using the single-pion exchange diagrams in the static limit. In our calculations, the Reid hard-core potential and Hulthen wave function for the deuteron were adopted, as providing the best description of the $v_{\mu} \mathrm{D}$ data.

All the fits are done with the CERN function minimization and error analysis package MINUIT [162,163]. The errors of the output parameters quoted below correspond to one and two standard deviation. As follows from the analysis, the deviation of the normalization factors $\mathscr{N}_{i}$ from unity for each data group $G_{i}$ does not exceed the doubled normalization uncertainty.

As a result of the analysis of the deuterium and hydrogen data, the best-fit value of $M_{A}^{\mathrm{D}}$ is found to be

$M_{A}^{\mathrm{D}}=1.003_{-0.083(0.108)}^{+0.083(0.109)} \mathrm{GeV}$

with the corresponding $\chi^{2} /$ ndf value of $127.5 /(117-7) \approx$ 1.16. Values in brackets in Eq. (5) and below are the twostandard-deviation $(95 \% \mathrm{CL})$ errors. The best-fit values of the running axial mass parameters are found as follows:

$$
\begin{aligned}
M_{0} & =1.052_{-0.094(0.113)}^{+0.095(0.114)} \mathrm{GeV}, \\
E_{0} & =278_{-111(131)}^{+130(158)} \mathrm{MeV},
\end{aligned}
$$

the parameter $M_{0}$ has been softly anchored to the best-fit value extracted from the fit to deuterium data. The inner and outer contours for both fits (6) and (7) indicate, respectively, the $68 \%$ and 95\% CL areas

with $\chi^{2} /$ ndf $=272.7 /(422-19) \approx 0.68$. The data for all nuclear targets were involved into this analysis.

As the next step, by taking into account that the SM RFG calculations well describe the high-energy data on all nuclear targets with the unique value of $M_{A}\left(\approx M_{A}^{\mathrm{D}} \approx 1 \mathrm{GeV}\right)$ [8], we add to the sum (3) the penalty term

$\left(M_{0}-1.003\right)^{2} / 0.083^{2}$

to constrain the bias of $M_{0}$ from $M_{A}^{\mathrm{D}}$. The final global fit performed with this constraint yields

$$
\begin{aligned}
M_{0} & =1.008 \pm 0.025(0.029) \mathrm{GeV}, \\
E_{0} & =331_{-54(64)}^{+57(69)} \mathrm{MeV}
\end{aligned}
$$

with $\chi^{2} /$ ndf $=277.6 /(422-19) \approx 0.69$.

Figure 3 shows the $1 \sigma$ and $2 \sigma$ confidence contours in the two-parameter plane, resulting from the three consecutive fits. It is in particular seen that the values of the parameters $M_{0}$ and $E_{0}$ obtained in the separate analyses used the $v_{\mu}, \bar{v}_{\mu}$, and $v_{\mu}+\bar{v}_{\mu}$ datasets are in agreement with each other within the $68 \%$ confidence contours; the agreement is worse for the best-fit values in the antineutrino case. It should be mentioned that the obtained values of $M_{A}^{\mathrm{D}}, M_{0}$, and $E_{0}$ are strictly speaking valid only within the set of the inputs adopted in our analyses, such as the parameters of the RFG model (see Eq. (2)) and parametrization of the vector form factors of the nucleon $\left(\mathrm{BBBA}_{25}(07)\right.$ model [4]). However, as our study shows, these values are quite stable relative to small variations of the input parameters.

Figure 4 illustrates the impact of the anchoring of $M_{0}$ on the $\chi^{2}$ values: the global minima of the $\chi^{2}$ for the stan- 

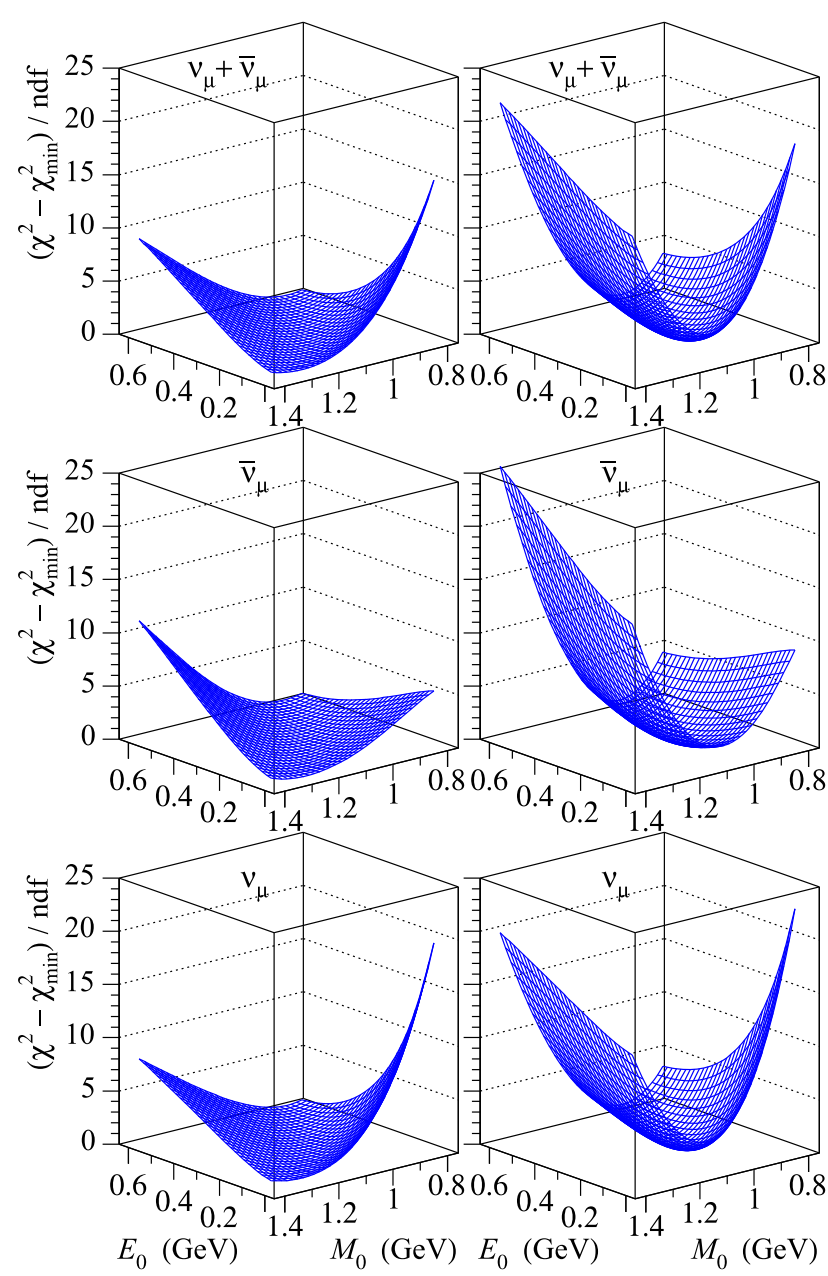

Fig. 4 Ratios $\left(\chi^{2}-\chi_{\min }^{2}\right) /$ ndf as functions of the free fitting parameters $E_{0}$ and $M_{0}$. The surfaces in left and right triplets of panels correspond to the global fits performed without and with "softly anchored" $M_{0}$, respectively

dard fits without the anchoring (shown in three left panels) is not very distinct, while after the soft anchoring of $M_{0}$ to the deuterium value $M_{A}^{\mathrm{D}}=1.003 \mathrm{GeV}$ the minima became distinctly visible.

A concentrated summary of comparison with the earlier data is presented in Figs. 5 and 6. Figure 5 displays the ratios of the total CCQE cross sections measured in different experiments to the respective predicted cross sections renormalized to the data subsets. The following experiments are presented: ANL 1969 [93], 1972 [94], 1973 [95], 1975 [96] (see also [146]), and 1977 [97,98]; BNL 1980 [79], FNAL 1983 [106] and 1984 [107], NuTeV 2004 [110]; SciBooNE 2009 [15,16], MiniBooNE 2010 [13], 2013 [14], and 2018 [165], LAr-TPC 2007 [135]; CERN HLBC 1966 [122], HLBC 1967 [123], GGM 1973 [76], 1977 [129], 1978 [164], and 1979 [131,132], BEBC 1990 [134], NOMAD 2008 [34]; IHEPITEP 1981 [136], 1982 [137], and 1985 [138,139]; IHEP SKAT 1981 [80], 1988 [141], and 1990 [142]; T2K INGRID
2015 [26]. The corresponding normalization factors, $\mathscr{N}$, are listed in Appendix Table 13 which is selfexplanatory. Notation used in panels (a), (b) and (c), (d) are the same as, respectively, in Figs. 28 and 29. Here and in all figures below, the data points marked with filled symbols indicate the data included in the global fit. The vertical error bars and heights of the shaded rectangles in panels (a) and (b) represent the total errors which include the experimental normalization uncertainties. Narrow double bands represent the effect of $1 \sigma$ and $2 \sigma$ uncertainties in determination of the parameters $M_{0}$ and (for nuclei heavier than deuterium) $E_{0}$; the uncertainties in the normalization factors are not shown since they are in general different for the different data subsets shown in the same panel. It is seen that the ratios for deuterium and hydrogen are slowly sensitive to the uncertainties of $M_{0}$ at $E_{v} \lesssim 200 \mathrm{GeV}$ and the ratios for the heavier nuclei are insensitive to the correlated uncertainties of $M_{0}$ and $E_{0}$ at $E_{v} \lesssim E_{0} \sim 0.3 \mathrm{GeV}$. The maximum sensitivity occurs at about $1 \mathrm{GeV}$ and does not exceeds $5 \%$. The normalization factors for the total cross sections measured in the experiments BNL 1980 [79], FNAL 1983 [106], MiniBooNE 2010 and 2013 [13,14], CERN GGM 1979 [132], CERN BEBC 1990 [134], and IHEP SKAT 1990 [142] were obtained from the differential distributions included into the global fit since the total CCQE cross sections of the listed experiments were not included into the global fit. The respective absolute data and model predictions are shown in Figs. 28, 29, and 30 without renormalization.

Similarly, Fig. 6 displays the ratios of the flux-weighted differential cross section $\left\langle d \sigma / d Q^{2}\right\rangle$ measured in different experiments to the corresponding predicted cross sections renormalized to the respective data subsets. The outer and inner vertical error bars represent the total errors of the experimental data and the total errors excluding the normalization uncertainties, respectively. In contrast to Fig. 5, the shaded bands represent the effect of the uncertainties in the normalization factors and do not include the uncertainties of the parameters $M_{0}$ and $E_{0}$. The boundaries of the bands are calculated as $\pm \Delta N_{\nu, \bar{\nu}} / N_{\nu, \bar{\nu}}$, where $\Delta N_{\nu, \bar{v}}$ and $N_{\nu, \bar{v}}$ are, respectively, the statistical uncertainty and best-fit value of the normalization factor. For the semi-sum of the $v_{\mu}$ and $\bar{v}_{\mu}$ cross sections measured in the IHEP-ITEP experiment $[138,139]$ (see panel (p)), the boundaries are calculated as

$\pm \frac{\Delta N_{v}\left\langle d \sigma_{v} / d Q^{2}\right\rangle+\Delta N_{\bar{v}}\left\langle d \sigma_{\bar{v}} / d Q^{2}\right\rangle}{N_{v}\left\langle d \sigma_{v} / d Q^{2}\right\rangle+N_{\bar{v}}\left\langle d \sigma_{\bar{v}} / d Q^{2}\right\rangle}$.

Comparisons between the corresponding absolute data and the $\mathrm{SMRFG}+M_{A}^{\text {run }}$ model predictions are shown (without renormalization) in several figures in Sect. 5.1 (for the MiniBooNE CCQE data) and in Appendix B (for the earlier data). The ratios shown in Fig. 6 are obtained by dividing the experimental data in each bin by the predicted cross section integrated over the bin and multiplied by the normalization fac- 


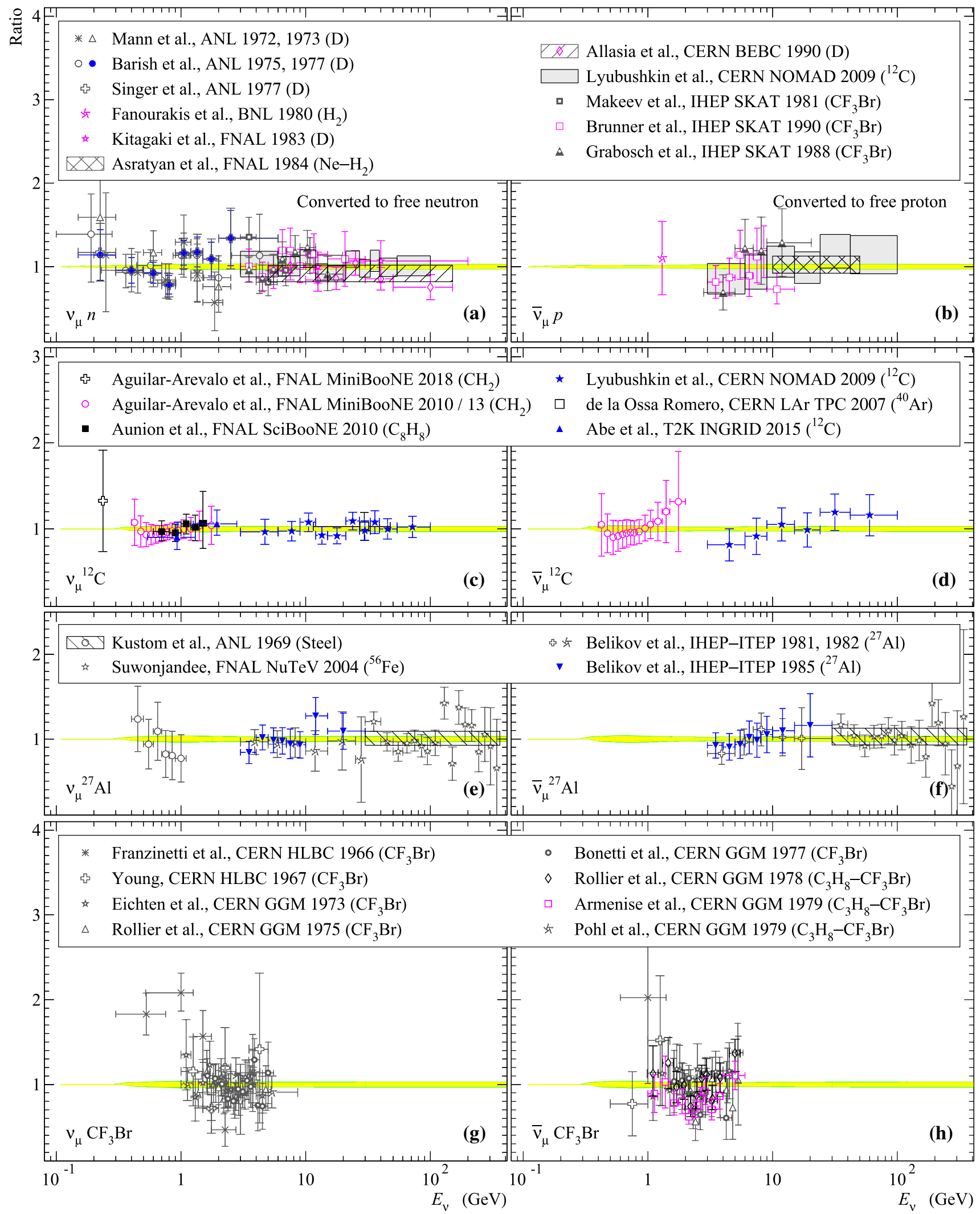

Fig. 5 Ratios of the total cross sections measured in different experiments to the corresponding predicted cross sections multiplied by the normalization factors $\mathscr{N}$ listed in Appendix Table 13. See text for references 


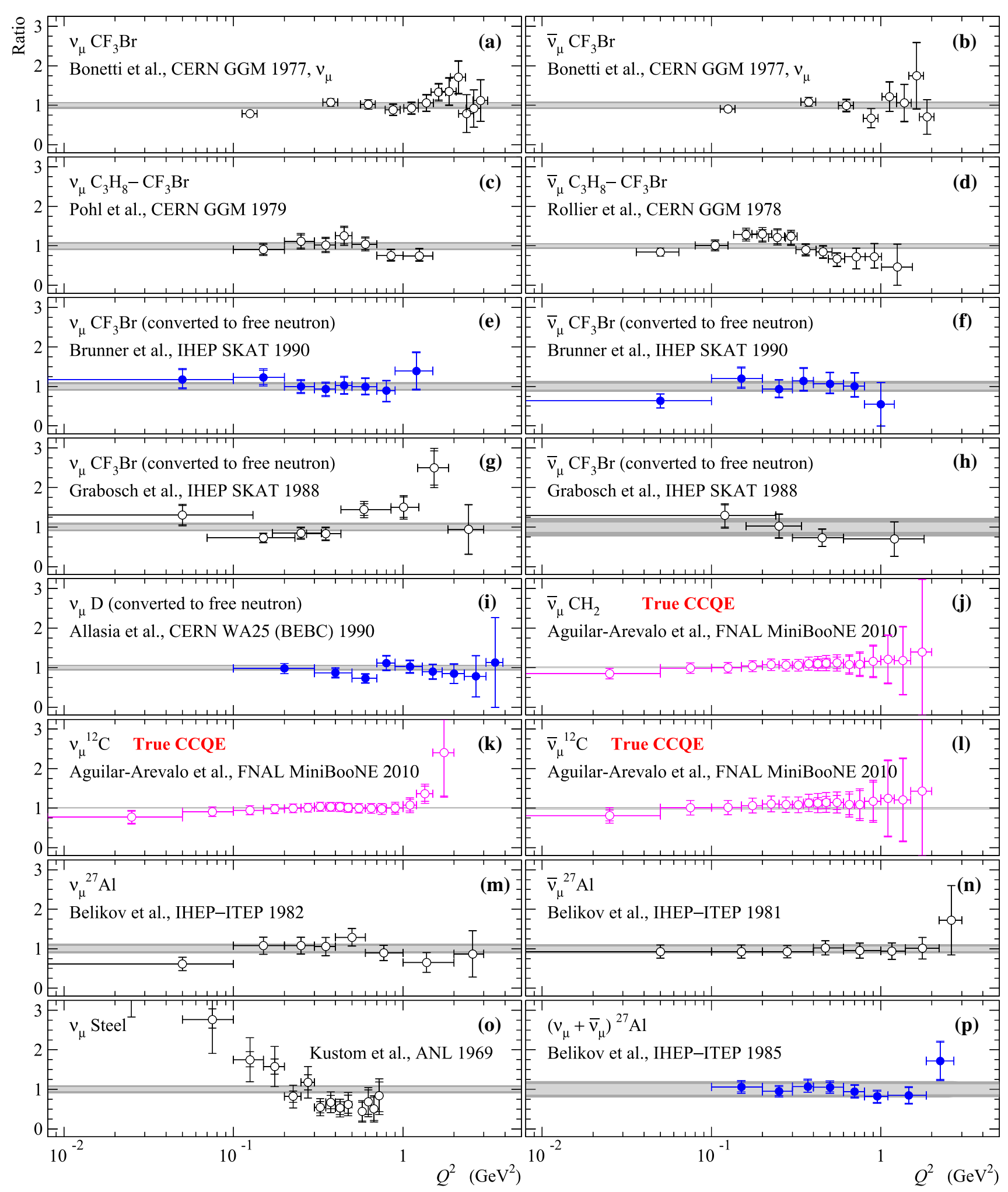

Fig. 6 Ratios of the differential cross sections $\left\langle d \sigma / d Q^{2}\right\rangle$ measured with ANL 1979 [93], FNAL MiniBooNE 2010 [13] 2013 [14], CERN GGM 1977 [129], 1978 [164], 1979 [131], BEBC [134], IHEP-ITEP 1981 [136], 1982 [137], 1985 [138,139], IHEP SKAT 1988 [141], and 1990 [142] to the corresponding predicted cross sections multiplied by the normalization factors $\mathscr{N}$ listed in Appendix Table 13. The shaded double bands indicate the $1 \sigma$ and $2 \sigma$ uncertainties in the normalization factors. Only the data shown by filled symbols were included in the global fit. See text for more details 


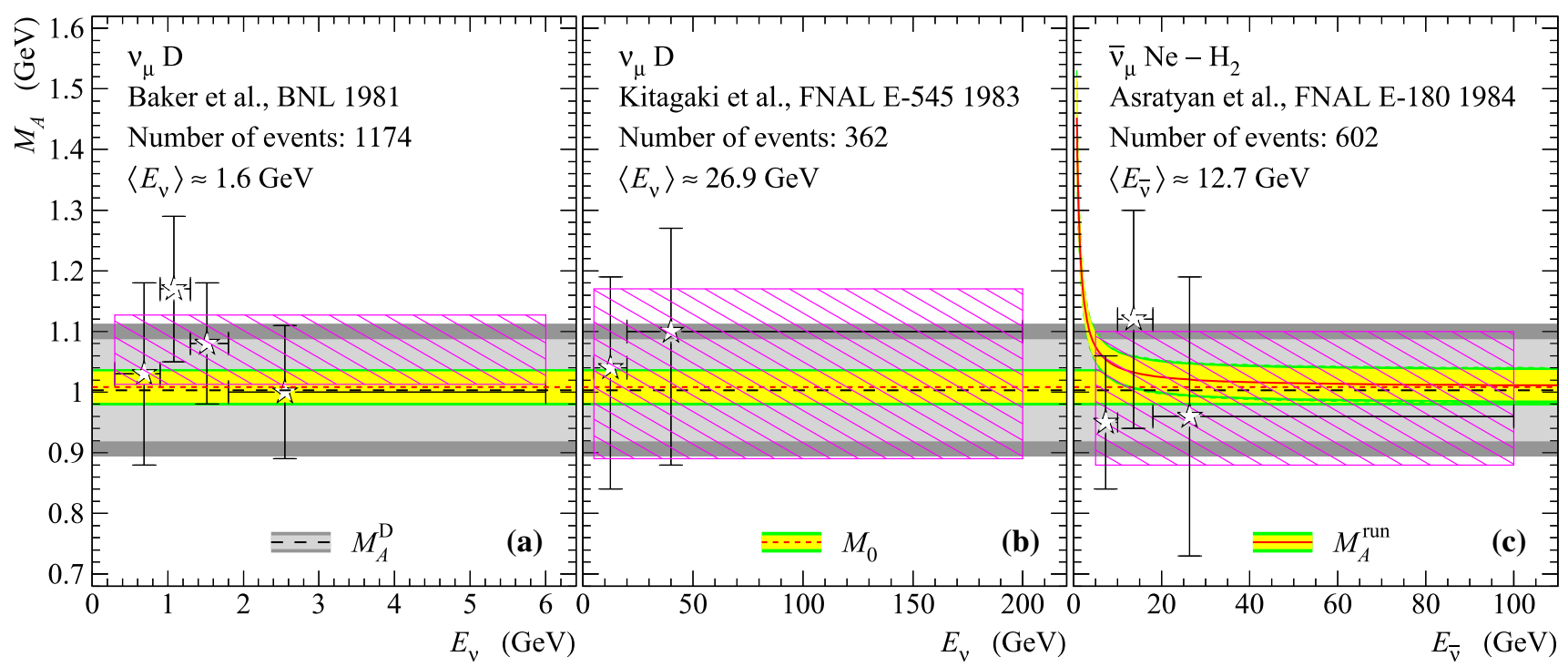

Fig. 7 Axial-vector mass, $M_{A}$, vs. (anti)neutrino energy extracted in the experiments BNL 1981 [101] (a), FNAL E-545 (1983) [106] (b), and FNAL E-180 (1984) [107] (c). Number of events and estimated mean energies of the beams for each of the experiments are given in the legends. Hatched rectangles show the values of $M_{A}( \pm 1 \sigma)$ averaged

tor (see Appendix Table 13) obtained for each experimental dataset from individual fits at fixed values of $M_{0}$ and $E_{0}$. The slight difference in the normalization factors for the MiniBooNE data shown in Appendix Table 13 and in Tables 1 and 2 is explained below in Sect. 5.1.

Figure 7 shows the current axial-vector mass, $M_{A}$, obtained in the three experiments at BNL [101] and FNAL $[106,107]$ performed with bubble chambers filled with deuterium $[101,106]$ and with a heavy neon-hydrogen mixture $(64 \% \mathrm{Ne})$ [107]. The data were extracted as functions of (anti)neutrino energy. Hatched regions indicate the average values of $M_{A}$ obtained for the full energy ranges: $\left\langle M_{A}\right\rangle=$ $1.070 \pm 0.057 \mathrm{GeV}$ for $E_{v}=0.3-6 \mathrm{GeV}$ (a), $1.05_{-0.16}^{+0.12} \mathrm{GeV}$ for $E_{v}=5-200 \mathrm{GeV}$ (b), and $0.99 \pm 0.11 \mathrm{GeV}$ for $E_{\bar{v}}=5-100 \mathrm{GeV}$ (c). The BNL result has been refined [104] with increased statistics, $\left\langle M_{A}\right\rangle=1.070_{-0.045}^{+0.040} \mathrm{GeV}$ for $E_{v}=0.5-6 \mathrm{GeV}$. The cited results are consistent with each other and with the low-energy data points from the Argonne 12-foot bubble chamber filled with hydrogen and deuterium $\left(M_{A}=0.95 \pm 0.09 \mathrm{GeV}\right.$ [97] and $1.00 \pm 0.05 \mathrm{GeV}$ [99]; the neutrino energy spectrum peaks at $0.5 \mathrm{GeV}$ ). It is important to keep in mind that the extractions in all these experiments were performed with different inputs.

No significant variation of $M_{A}$ with energy is seen within rather wide energy range. The straight lines and curve with shaded bands represent the best-fit values of $M_{A}^{\mathrm{D}}, M_{0}$, and $M_{A}^{\text {run }}$ with corresponding $1 \sigma$ and $2 \sigma$ uncertainties drawn according to Eqs. (5) and (7). As is seen, the straight lines (representing $M_{A}^{\mathrm{D}}$ and $M_{0}$ ), are consistent with each other and over the full energy ranges (see text). Shaded bands around straight lines and around the curve in panel $\mathbf{c}$ indicate the $1 \sigma$ and $2 \sigma$ confidence intervals for the best-fitted values of $M_{A}^{\mathrm{D}}, M_{0}$, and $M_{A}^{\text {run }}$ obtained in the global fits (see Eqs. (5) and (7))

with the deuterium data. They are also in very good agreement with the results of the former analyses [3-8]. The result of Ref. [107] (Ne- $\mathrm{H}_{2}$ mixture) is formally consistent with both constant and running axial masses with about the same statistical significance. More comprehensive discussion of the earlier and current data on $M_{A}$ extractions is presented in Ref. [166].

\section{GENIE 3 features}

Let us discuss some features of the GENIE 3 package that are essential for further consideration. The package provides a set of out-of-the-box comprehensive model configurations instead of a single "default" one, which has been a subject of customization by an user in the previous versions of GENIE. A set of models with their preset configurations is called "tune". ${ }^{2}$ The advantages of this innovation is in that the user gets a self-consistent combination of the physical models in which a double-counting is excluded. Each tune is characterized by detailed MC comparisons with the data to which the input parameters were adjusted. Among the models presented in each tune, the most important are the models for elastic, quasielastic, and deep-inelastic scattering and resonance meson production. The tunes also include models for coherent and diffractive production of pions on nuclei, which make

\footnotetext{
2 A full list of GENIE 3 model configurations and tunes is maintained in URL: http://tunes.genie-mc.org.
} 
Table 1 The values of $\chi_{\mathrm{st}}^{2} / \mathrm{ndf}$, $\chi_{1}^{2} /$ ndf, $\chi_{\mathscr{N}}^{2} /(\mathrm{ndf}-1)$, and $\mathscr{N}$, evaluated with several models for the total, single-differential, and double-differential CCQE and CCQE-like cross sections for $v_{\mu}$ scattering from carbon as measured by MiniBooNE [13]. Respective numbers are shown before (CCQE) and after (CCQE-like) slashes. The results for the GIBUU, SuSAv2-MEC, and SuSAM* models are only available for the CCQE cross sections. The relevant data and selected model predictions are shown in Figs. 13a, b, 14a, b, and 15 (see also Fig. 28a). The meaning of the listed quantities is explained by Eqs. (8)-(12) (implying in this instance only diagonal covariances). The ndf (second column) represents the number of experimental bins for each calculation. The relative normalization uncertainty $\delta=10.7 \% / 9.8 \%$ for the CCQE/CCQE-like cross sections is taken the same for all data subsets. Also shown the $\chi^{2} \mathrm{~s}$ and normalization factors, $\mathscr{N}$, for the joint MiniBooNE neutrino CCQE and CCQE-like datasets including the measurement of $v_{\mu}{ }^{12} \mathrm{C} \rightarrow \mu^{-} X$ cross section at $E_{v}=236 \mathrm{MeV}$ reported in Ref. [165]. Here and in the tables below, three digits in mantissa of $\mathscr{N} \mathrm{s}$ are displayed to distinguish differences caused by the two FSI models

\begin{tabular}{|c|c|c|c|c|c|}
\hline Model & ndf & $\frac{\chi_{\mathrm{st}}^{2}}{\mathrm{ndf}}$ & $\frac{\chi_{1}^{2}}{\mathrm{ndf}}$ & $\frac{\chi_{\mathscr{N}}^{2}}{\text { ndf-1 }}$ & $\mathscr{N}$ \\
\hline \multicolumn{6}{|l|}{$\sigma_{\text {tot }}^{\mathrm{QE}}$} \\
\hline $\mathrm{SMRFG}+M_{A}^{\text {run }}(\mathrm{hA} 2018)$ & 14 & $0.07 / 0.05$ & $0.13 / 0.08$ & $0.07 / 0.08$ & $0.972 / 0.990$ \\
\hline with monoenergetic point & 15 & $0.09 / 0.07$ & $0.14 / 0.10$ & $0.10 / 0.10$ & $0.973 / 0.991$ \\
\hline $\mathrm{SMRFG}+M_{A}^{\mathrm{run}}(\mathrm{hN} 2018)$ & 14 & $0.07 / 0.06$ & $0.13 / 0.09$ & $0.07 / 0.09$ & $0.972 / 0.993$ \\
\hline with monoenergetic point & 15 & $0.09 / 0.07$ & $0.14 / 0.11$ & $0.10 / 0.11$ & $0.973 / 0.994$ \\
\hline G18_10a_02_11a & 14 & $0.78 / 0.99$ & $1.39 / 1.88$ & $0.44 / 0.45$ & $1.129 / 1.139$ \\
\hline with monoenergetic point & 15 & $0.78 / 0.98$ & $1.35 / 1.81$ & $0.44 / 0.45$ & $1.131 / 1.140$ \\
\hline G18_10b_02_11a & 14 & $0.78 / 1.04$ & $1.39 / 1.96$ & $0.44 / 0.45$ & $1.129 / 1.143$ \\
\hline with monoenergetic point & 15 & $0.79 / 1.03$ & $1.36 / 1.88$ & $0.44 / 0.45$ & $1.131 / 1.144$ \\
\hline SuSAv2-MEC 2016 & 14 & 0.08 & 0.11 & 0.12 & 1.004 \\
\hline SuSAM $^{*} 2019$ & 14 & 0.46 & 0.89 & 0.12 & 1.113 \\
\hline \multicolumn{6}{|l|}{$d \sigma / d Q_{\mathrm{QE}}^{2}$} \\
\hline $\mathrm{SMRFG}+M_{A}^{\mathrm{run}}(\mathrm{hA} 2018)$ & 17 & $0.23 / 0.17$ & $0.81 / 0.75$ & $0.68 / 0.56$ & $1.017 / 1.017$ \\
\hline $\mathrm{SMRFG}+M_{A}^{\mathrm{run}}(\mathrm{hN} 2018)$ & 17 & $0.23 / 0.19$ & $0.74 / 0.87$ & $0.68 / 0.54$ & $1.013 / 1.022$ \\
\hline G18_10a_02_11a & 17 & $1.43 / 1.58$ & $19.3 / 22.0$ & $1.66 / 1.31$ & $1.203 / 1.195$ \\
\hline G18_10b_02_11a & 17 & $1.43 / 1.75$ & $19.3 / 24.0$ & $1.68 / 1.32$ & $1.203 / 1.206$ \\
\hline GIBUU 2016 & 16 & 0.63 & 3.19 & 1.25 & 0.948 \\
\hline SuSAM* 2019 & 17 & 4.08 & 28.1 & 12.0 & 1.198 \\
\hline \multicolumn{6}{|l|}{$d^{2} \sigma / d E_{\mu} d \cos \theta_{\mu}$} \\
\hline $\mathrm{SMRFG}+M_{A}^{\mathrm{run}}(\mathrm{hA} 2018)$ & 137 & $0.20 / 0.21$ & $0.27 / 0.26$ & $0.25 / 0.26$ & $0.980 / 1.000$ \\
\hline forward scattering & 105 & $0.20 / 0.23$ & $0.26 / 0.29$ & $0.27 / 0.29$ & \\
\hline backward scattering & 32 & $0.18 / 0.13$ & $0.28 / 0.16$ & $0.19 / 0.16$ & \\
\hline $\mathrm{SMRFG}+M_{A}^{\mathrm{run}}(\mathrm{hN} 2018)$ & 137 & $0.20 / 0.23$ & $0.27 / 0.28$ & $0.25 / 0.28$ & $0.977 / 1.008$ \\
\hline forward scattering & 105 & $0.20 / 0.26$ & $0.25 / 0.32$ & $0.26 / 0.31$ & \\
\hline backward scattering & 32 & $0.19 / 0.14$ & $0.31 / 0.17$ & $0.19 / 0.18$ & \\
\hline G18_10a_02_11a & 137 & $0.61 / 0.61$ & $0.97 / 1.05$ & $0.77 / 0.56$ & $1.080 / 1.117$ \\
\hline forward scattering & 105 & $0.55 / 0.63$ & $0.94 / 1.12$ & $0.55 / 0.40$ & \\
\hline backward scattering & 32 & $0.78 / 0.50$ & $1.02 / 0.74$ & $1.45 / 1.02$ & \\
\hline G18_10b_02_11a & 137 & $0.61 / 0.64$ & $0.97 / 1.12$ & $0.77 / 0.51$ & $1.079 / 1.131$ \\
\hline forward scattering & 105 & $0.55 / 0.69$ & $0.94 / 1.21$ & $0.56 / 0.37$ & \\
\hline backward scattering & 32 & $0.77 / 0.45$ & $1.02 / 0.71$ & $1.44 / 0.88$ & \\
\hline GIBUU 2016 & 137 & 3.52 & 3.82 & 3.53 & 0.916 \\
\hline forward scattering & 105 & 0.49 & 0.60 & 0.82 & \\
\hline backward scattering & 32 & 13.4 & 14.2 & 12.3 & \\
\hline SuSAv2-MEC 2016 & 137 & 1.05 & 1.69 & 1.46 & 1.086 \\
\hline forward scattering & 105 & 0.81 & 1.15 & 1.19 & \\
\hline backward scattering & 32 & 1.78 & 3.38 & 2.30 & \\
\hline SuSAM* 2019 & 137 & 3.08 & 4.72 & 3.71 & 1.208 \\
\hline forward scattering & 105 & 1.78 & 2.71 & 1.99 & \\
\hline backward scattering & 32 & 7.24 & 11.1 & 9.10 & \\
\hline \multicolumn{6}{|c|}{ Joint dataset including monoenergetic point } \\
\hline $\mathrm{SMRFG}+M_{A}^{\mathrm{run}}(\mathrm{hA} 2018)$ & 169 & & $0.31 / 0.30$ & $0.31 / 0.28$ & $1.002 / 1.011$ \\
\hline $\mathrm{SMRFG}+M_{A}^{\mathrm{run}}(\mathrm{hN} 2018)$ & 169 & & $0.30 / 0.33$ & $0.31 / 0.30$ & $0.999 / 1.016$ \\
\hline G18_10a_02_11a & 169 & & $2.84 / 3.32$ & $1.04 / 0.83$ & 1.158/1.169 \\
\hline G18_10b_02_11a & 169 & & $2.84 / 3.60$ & $1.05 / 0.79$ & $1.158 / 1.181$ \\
\hline
\end{tabular}


Table 2 Same as in Table 1 but for the $\mathrm{CCQE} \bar{v}_{\mu} \mathrm{CH}_{2}$ and $\bar{v}_{\mu}{ }^{12} \mathrm{C}$ cross sections [14]; respective numbers are shown before $\left(\mathrm{CH}_{2}\right)$ and after $\left({ }^{12} \mathrm{C}\right)$ slashes. Here and below, the abbreviation "G18_10a/b_02_11a" means that the listed $\chi^{2} \mathrm{~s}$ and $\mathscr{N} \mathrm{s}$ are the same (within the accepted rounding-off conventions) for the G18_10a_02_11a and G18_10b_02_11a tunes. The results for the GIBUU, SuSAv2-MEC, and SuSAM* models are only available for the CCQE cross sections on carbon. The relevant data and selected model predictions are shown in Figs. 13c, d, 14c, d, and 16 (see also Fig. 28b). The normalization uncertainties, $\delta$, for each data subset are shown in parentheses

\begin{tabular}{|c|c|c|c|c|c|}
\hline Model & ndf & $\frac{\chi_{\mathrm{st}}^{2}}{\mathrm{ndf}}$ & $\frac{\chi_{1}^{2}}{\mathrm{ndf}}$ & $\frac{\chi_{\mathscr{N}}^{2}}{\text { ndf-1 }}$ & $\mathscr{N}$ \\
\hline \multicolumn{6}{|l|}{$\sigma_{\text {tot }}^{\mathrm{QE}}(\delta=13.0 \% / 17.4 \%)$} \\
\hline $\mathrm{SMRFG}+M_{A}^{\mathrm{run}}(\mathrm{hA} 2018)$ & 14 & $0.08 / 0.08$ & $0.13 / 0.12$ & $0.13 / 0.13$ & $0.991 / 0.994$ \\
\hline $\mathrm{SMRFG}+M_{A}^{\text {run }}(\mathrm{hN} 2018)$ & 14 & $0.09 / 0.08$ & $0.13 / 0.12$ & $0.14 / 0.13$ & $0.991 / 0.993$ \\
\hline G18_10a/b_02_11a & 14 & $0.43 / 0.41$ & $0.82 / 0.75$ & $0.14 / 0.16$ & $1.122 / 1.161$ \\
\hline SuSAv2-MEC 2016 & 14 & 0.10 & 0.15 & 0.09 & 1.050 \\
\hline SuSAM* 2019 & 14 & 0.16 & 0.29 & 0.07 & 1.093 \\
\hline \multicolumn{6}{|l|}{$d \sigma / d Q_{\mathrm{QE}}^{2}(\delta=12.9 \% / 17.2 \%)$} \\
\hline $\mathrm{SMRFG}+M_{A}^{\mathrm{run}}(\mathrm{hA} 2018)$ & 17 & $0.12 / 0.13$ & $0.46 / 0.45$ & $0.47 / 0.37$ & $1.007 / 1.026$ \\
\hline $\mathrm{SMRFG}+M_{A}^{\mathrm{run}}(\mathrm{hN} 2018)$ & 17 & $0.12 / 0.13$ & $0.46 / 0.45$ & $0.46 / 0.37$ & $1.007 / 1.025$ \\
\hline G18_10a_02_11a & 17 & $0.48 / 0.40$ & $4.85 / 3.91$ & $0.55 / 0.37$ & $1.128 / 1.169$ \\
\hline G18_10b_02_11a & 17 & $0.48 / 0.40$ & $4.84 / 3.90$ & $0.55 / 0.37$ & $1.128 / 1.169$ \\
\hline GIBUU $2016(\mathscr{T}=1)$ & 14 & 0.54 & 1.59 & 0.95 & 1.062 \\
\hline SuSAM* $^{*} 2019$ & 17 & 1.85 & 9.05 & 5.57 & 1.179 \\
\hline \multicolumn{6}{|l|}{$d^{2} \sigma / d E_{\mu} d \cos \theta_{\mu}(\delta=13.0 \% / 17.2 \%)$} \\
\hline $\mathrm{SMRFG}+M_{A}^{\mathrm{run}}(\mathrm{hA} 2018 \& \mathrm{hN} 2018)$ & $78 / 75$ & $0.39 / 0.39$ & $0.56 / 0.57$ & $0.55 / 0.57$ & $0.991 / 0.992$ \\
\hline forward scattering & $70 / 67$ & $0.34 / 0.34$ & $0.52 / 0.53$ & $0.52 / 0.53$ & \\
\hline backward scattering & 8 & $0.80 / 0.82$ & $0.84 / 0.89$ & $0.80 / 0.85$ & \\
\hline G18_10a/b_02_11a & $78 / 75$ & $0.45 / 0.38$ & $0.68 / 0.62$ & $0.47 / 0.37$ & $1.077 / 1.110$ \\
\hline forward scattering & $70 / 67$ & $0.44 / 0.37$ & $0.68 / 0.62$ & $0.45 / 0.35$ & \\
\hline backward scattering & 8 & $0.49 / 0.44$ & $0.53 / 0.51$ & $0.55 / 0.46$ & \\
\hline GIBUU $2016(\mathscr{T}=1)$ & $78 / 75$ & 0.44 & 0.73 & 0.41 & 1.127 \\
\hline forward scattering & $70 / 67$ & 0.42 & 0.72 & 0.39 & \\
\hline backward scattering & 8 & 0.58 & 0.68 & 0.51 & \\
\hline SuSAv2-MEC 2016 & $78 / 75$ & 0.57 & 1.00 & 0.83 & 1.088 \\
\hline forward scattering & $70 / 67$ & 0.56 & 1.01 & 0.84 & \\
\hline backward scattering & 8 & 0.60 & 0.70 & 0.60 & \\
\hline SuSAM* 2019 & $78 / 75$ & 1.40 & 2.51 & 1.60 & 1.237 \\
\hline forward scattering & $70 / 67$ & 1.38 & 2.55 & 1.58 & \\
\hline backward scattering & 8 & 1.38 & 1.58 & 1.30 & \\
\hline \multicolumn{6}{|l|}{ Joint dataset $\left(\delta_{\text {eff }}=13.0 \% / 17.3 \%\right)$} \\
\hline $\mathrm{SMRFG}+M_{A}^{\text {run }}(\mathrm{hA} 2018)$ & $109 / 106$ & & $0.48 / 0.49$ & $0.49 / 0.49$ & $1.000 / 1.011$ \\
\hline $\mathrm{SMRFG}+M_{A}^{\text {run }}(\mathrm{hN} 2018)$ & $109 / 106$ & & $0.48 / 0.49$ & $0.49 / 0.49$ & $1.000 / 1.010$ \\
\hline G18_10a_02_11a & $109 / 106$ & & $1.34 / 1.15$ & $0.46 / 0.36$ & $1.111 / 1.149$ \\
\hline G18_10b_02_11a & $109 / 106$ & & $1.34 / 1.15$ & $0.46 / 0.35$ & $1.111 / 1.149$ \\
\hline
\end{tabular}

a small contribution to the CCQE-like background. Common to all tunes are the models for secondary interactions of hadrons inside nuclei. In addition to INTRANUKE - the intranuclear transport simulation subpackage, which was the default in the GENIE releases prior to 3.0.0, its updated ver- sion (hereinafter referred to as INTRANUKE 2018) is added. The INTRANUKE 2018 consists of two models: hA 2018 and hN2018, whereas the former version of INTRANUKE included the only model called $\mathrm{hA}$. The main distinctions between the models hA, hA 2018, and hN 2018 are discussed below in some detail. 


\subsection{Final state interaction models}

Neutrino interaction with a nucleon bound in a nucleus may produce secondary hadrons which then may interact (elastically or inelastically) on their way out of the nucleus. During the re-scattering, the secondaries can be absorbed, change their charge and 4-momentum, or produce new particles; besides multiple nucleons can be formed, causing spallation of the nucleus. The set of all these processes is commonly referred to as final state interaction (FSI) and its modeling is an important ingredient of any neutrino event generator $[2,74]$. It is amply clear that FSI change the distributions in outgoing nucleon variables, and, besides, may indirectly affect the distributions in terms of leptonic variables, because of experimental cuts imposed on the final-state nucleon variables affecting the event selection. One of the most important aspect of the FSI effects is in mimicking the CCQE topology by inelastic processes (e.g. pion production and absorption) inside the nucleus.

The intranuclear cascade (INC) models treat the hadrons propagating in a nucleus as free classical particles between two successive collisions on isolated nucleons bound in a potential well and undergoing Fermi motion. More sophisticated INC models (as, e.g., the model by Salcedo et al. Ref. [167]) can account for the real part of hadron self-energy potential to simulate their elastic scattering on nucleons. In INC models, the probability per unit length of a certain reaction type (elastic scattering, charge exchange, pion absorption, pion production, etc. [168-170]) is fundamental value which is determined by the corresponding cross sections and nuclear density. Although these models describe hadron scattering on nuclei quite well, the more accurate results can be reached by quantum-mechanical calculations (using a "black disk" approximation for quantum diffraction), when the interference between reaction types is accounted by summing of appropriate amplitudes. A pragmatic drawback of the quantum-mechanical calculations is the requirement of large computational resources, while the INC models are not so resource-intensive and in addition have the advantage that one can much easier trace propagation of hadrons in the nuclear medium.

The intranuclear transport of hadrons and $\gamma$-quanta and their scattering are managed in GENIE 3 [170] by two INC simulation subpackages - INTRANUKE (deprecated) and INTRANUKE 2018. To determine the mean free paths of hadrons and $\gamma \mathrm{s}$ in nuclear medium, both INC subpackages use as input data the empirical cross sections for $p, \pi, K$, and $\gamma$ interactions with bare nucleons and nuclei; the subpackage INTRANUKE 2018 uses partially updated data and nuclear corrections of various kinds to the free nucleon cross sections. The nuclear densities are described by Gaussian or modified Gaussian shapes for nuclei with $A<20$, and for heavier nuclei - by the Wood-Saxon density distribution dependent on surface thickness of the nucleus.

The hA and hA2018 models are comparatively simple empirical models based on the total cross section data for different atomic nuclei, from helium to lead. Using these data, both models evaluate the probabilities of absorbing, generating, or recharging of nucleons, pions, and kaons with kinetic energies up to $1.2 \mathrm{GeV}$. In the low-energy region of (50-300) $\mathrm{MeV}$, there are sufficient data for cascade modeling [171-175] but at higher energies, where the pion production probability becomes essential, the available data are rather fragmentary and thus the phenomenological cascadeexciton model CEM03 [176-178] is applied. The cascade is modeled on an iron nucleus and the re-scaling factor $\propto A^{2 / 3}$ is used to determine the cross sections on nuclei different from iron. The isospin symmetry is assumed in the models to recalculate the cross sections for $\pi^{0}$ from the charged pion data. The pion absorption is split into couple of different simulations: the absorption by two nucleons (by using the $\pi d \rightarrow N N$ data) and multi-nucleon absorption; the split probability is governed by empirical data. If there are two or more hadrons in the final state, the code distributes them evenly over the full phase space.

The hA model can generate the hadron-nucleus elastic interactions when there is the probability that the scattered hadron gains energy due to the energy lost by the recoil nucleus. Since interaction of this type cannot be simulated in the INC spirit, it is added through an empirical model [179]. Following recommendations of Ref. [112] the effect of energy gain by the scattered particle has been turned off in the hA 2018 model because, it is assumed to be responsible for the discrepancy with the recent MINER $v A$ data [114]. Other distinctions between the hA and hA 2018 models consist in different values of the nucleon binding energies and in a $5 \mathrm{MeV}$ correction applied for compound hadron cluster in the new model. For both models, in simulating the pion absorption, the binding energy is treated as a tunable parameter (for simplicity common to all nuclei) to fit the inclusive pion-nucleus scattering data from Ref. [180]. The tuned value adopted in the hA (hA 2018) model is close to that of the SM RFG model for iron (carbon).

The hN2018 model is fully INC model which allows to simulate all type of reactions for any nucleus and does not have the limitations peculiar to the hA2018 model. It utilizes the partial wave analysis of available data on the cross sections for $\pi N, K N$, and $N N$ interactions as provided by the Scattering Analysis Interactive Dial-in (SAID) program [181-183]. For pions with kinetic energy below $350 \mathrm{MeV}$ the hN2018 uses the method of Ref. [167] which is based on " $\Delta$ dominance" (the pion-nucleus interactions are simulated through $\Delta(1232)$ excitation) and combines a microscopic field-theoretical calculation of the intrinsic probabilities for each reaction channels and a simulation procedure to 
follow the history of each pion. The " $\Delta$ dominance" model exploits the many-body techniques. The calculations are performing in infinite nuclear matter with later correction for finite nuclei via the local density approximation and finiterange effects. The hN2018 model, unlike the hA 2018 one, allows to simulate pre-equilibrium and compound nuclear states and the pion absorption is simulated as absorption on separate nucleons rather than on deuteron. The simulations of hadron transport through the nuclear medium have some common features in the hN2018 and hA 2018 models. In particular, the interaction points in both models is estimated through a mean-field potential, the probability of interaction is determined in a similar way as value proportional to the mean free path, but the reaction channel is chosen on the basis of different input data.

\subsection{G18 tunes}

For comparison purposes, we will deal with two of several comprehensive theory-driven GENIE 3 physics tunes designated as G18_10a_02_11a and G18_10b_02_11a (for short, these tunes will be sometimes abbreviated to G18), incorporating, respectively, the hA 2018 and hN2018 FSI models discussed in Sect. 4.1. The remaining physical content of both tunes is quite the same. The CCQE sector is based on the model by Nieves et al. [184] which is, in turn, an extension of the results of Refs. [185-187], where the quasielastic contribution to the inclusive electron and neutrino scattering on nuclei was studied. The model is founded on a many-body expansion of the gauge boson absorption modes that includes $1 p 1 h, 2 p 2 h$, and even $3 p 3 h$ excitation mechanisms, as well as the excitation of $\Delta$ isobars. The $1 p 1 h$ contribution is included within a local Fermi gas (LFG) picture incorporating several nuclear corrections, such as correct energy balance, long-range nuclear (RPA) correlations, Coulomb distortion, nuclear medium polarization, and dressing the nucleon propagators.

The inelastic interactions (including coherent and diffractive meson production) in the "G18" tunes are handled in an almost similar fashion as for the $M_{A}^{\text {run }}$ model. The main differences are in the Berger-Sehgal model [89] for the resonance pion neutrinoproduction; in particular, the tunes use a bit refined version of the Rein-Sehgal normalization of the Breit-Wigner terms, and the vector and axial transition form factor model from Ref. [188]. In contrast to the KLN-BS model, no Pauli blocking is included.

All adjustable parameters, such as quasielastic and resonance axial-vector masses $(0.961 \mathrm{GeV}$ and $1.065 \mathrm{GeV}$, respectively), cut in the invariant hadronic mass (1.928 GeV), specifications applied in the NEUGEN neutrino generator [189] to the hadron multiplicity distributions (to avoid double counting of the DIS and resonance contributions), reweighing factors, etc. were tuned using all available data on the
$\mathrm{CCQE}, \mathrm{CC} 1 \pi, \mathrm{CC} 2 \pi$, and $\mathrm{CC}$ inclusive cross-sections and multiplicities. In our opinion, the most controversial point is the $12 \%$ overall reduction of the resonance production cross sections. Recall that the $M_{A}^{\text {run }}$ model does not use any reweighing factors and utilizes the common GENIE 3 set of parameters excluding those mentioned in Sect. 2.

Finally, it should be mentioned that all calculations with GENIE were done using the computational resources provided by the JINR cloud service [190,191].

\section{Comparison with recent data}

In this section, we compare in detail the contemporary comprehensive CCQE and CCQE-like data with the related predictions of the $\mathrm{SMRFG}+M_{A}^{\text {run }}$ model (in conjunction with hA 2018 and hN2018 FSI) and two G18 physics tunes. In addition to that, we consider three more competing phenomenological models: GIBUU [192], SuSAv2-MEC $[65,66]$, and SuSAM* $[68,193]$.

The GIBUU model is a part of the GIBUU (Giessen Boltzmann-Uehling-Uhlenbeck) project [194] and is an implementation of quantum-kinetic transport theory describing space-time evolution of a many-particle system in a relativistic mean-field potential. The model is based on a refinement of the nuclear ground state and of $2 p 2 h$ interactions by using an empirical structure function that has separate momentum and energy dependence adjusted from electron scattering data. The $2 p 2 h$ contribution is dependent on the target isospin, $\mathscr{T}$. Nucleon momenta come from an LFG distribution and the secondaries (also resonances) propagate through the residual nucleus in a nuclear potential that is consistent between initial and final state

The SuSAv2-MEC model represents a fully relativistic approach, which exploits the scaling and superscaling [195] features of inclusive electron scattering data [196] in order to predict neutrino-nucleus observables. The model is enhanced with the inclusion of relativistic mean-field theory effects and $2 p 2 h$ axial and vector contributions to weak response functions in a relativistic Fermi gas. Recently the SuSAv2-MEC model has been incorporated into the GENIE package [197], but in the present analysis we use only the original calculations from Ref. [65].

The SuSAM* model is another representative of the superscaling approach, based on a scaling function extracted from a selection of electron-nucleus scattering data [198], and an effective nucleon mass inspired by the relativistic meanfield model; the effective mass phenomenologically incorporates the enhancement of the transverse current produced by the relativistic mean field and the scaling function merges nuclear effects beyond the impulse approximation, such as short-range correlations (responsible for tails in the scaling function). Note that the results of this model are usually pre- 
sented as (rather wide) bands representing uncertainties in the implemented parameter set. In our estimations of the $\chi^{2}$ values (in exactly the same way as for other models) we use the central (rather than bin-averaged) values of the predicted cross sections and do not take into account the intrinsic model uncertainties. So the obtained $\chi^{2}$ values are significantly overestimated for the SuSAM* model and should be treated with care. A few more models will be discussed in Sects. 5.2 and 5.3.

In order to provide the grounds for a quantitative comparison, in the subsequent discussion we employ the following definitions:

$$
\begin{aligned}
\chi_{\mathrm{st}}^{2} & =(\mathbf{E}-\mathbf{T})^{T} \mathbf{W}^{-1}(\mathbf{E}-\mathbf{T}), \\
\chi_{N}^{2} & =(\mathbf{E}-N \mathbf{T})^{T} \tilde{\mathbf{W}}^{-1}(\mathbf{E}-N \mathbf{T})+\frac{(N-1)^{2}}{\delta^{2}}, \\
\chi_{\log }^{2} & =(\ln \mathbf{E}-\ln \mathbf{T})^{T} \tilde{\mathbf{V}}^{-1}(\ln \mathbf{E}-\ln \mathbf{T}) .
\end{aligned}
$$

Here $\mathbf{E}$ and $\mathbf{T}$ are, respectively, the vectors of bin-averaged experimental data, $E_{i}$, and corresponding model predictions, $T_{i}$; $\ln \mathbf{E}$ and $\ln \mathbf{T}$ are, respectively, the vectors with the components $\ln \left(E_{i}\right)$ and $\ln \left(T_{i}\right) ; \mathbf{W}=\left\|W_{i j}\right\|$ is the full covariance matrix, $\widetilde{\mathbf{W}}=\mathbf{W}-\mathbf{W}_{\text {norm, }}$, where $\mathbf{W}_{\text {norm }}$ is the matrix of covariances due to the normalization uncertainties; $\mathbf{V}=\left\|W_{i j} / E_{i} E_{j}\right\| ; \delta_{i}$ is the (dimensionless) normalization uncertainty. Note that $\chi_{\log }^{2} \approx \chi_{\mathrm{st}}^{2}$ as $\left|T_{i}-E_{i}\right| \ll E_{i}$ for all $i$. We will mainly use the log-normal $\chi^{2} \mathrm{~s}(10)$ for comparisons with the T2K and MINER $v \mathrm{~A}$ data. Very isolated singular contributions to $\chi_{\log }^{2}$ are merely excluded from the analysis.

The normalization factor in Eq. (9) is defined by the minimization condition $\partial \chi_{N}^{2} / \partial N=0$ :

$N=\mathscr{N}=\frac{1+\delta^{2} \mathbf{T}^{T} \tilde{\mathbf{W}}^{-1} \mathbf{E}}{1+\delta^{2} \mathbf{T}^{T} \tilde{\mathbf{W}}^{-1} \mathbf{T}}$

(cf. with Eq. (4)). From Eqs. (9) and (11) it follows that

$\chi_{\mathscr{N}}^{2}=\chi_{1}^{2}-(\mathscr{N}-1)^{2}\left(\frac{2}{\delta^{2}}+\mathbf{T}^{T} \tilde{\mathbf{W}}^{-1} \mathbf{T}\right)$.

It is clear that "good" values of $\chi_{\mathscr{N}}^{2}$ would indicate agreement in shape between the data and model prediction provided that $|\mathscr{N}-1| \lesssim \delta$. Unfortunately the formal solution (11) (with some modifications due to the lack of the matrix $\mathbf{W}_{\text {norm }}$ ) as a rule appears to be unphysical for the T2K data on hydrocarbon and MINERvA data (see Sect. 5.2.2 and also Ref. [199] where similar problem is discussed). Moreover, owing to strong correlations the standard $\chi^{2}$ values for the T2 $\mathrm{K} \mathrm{C}_{8} \mathrm{H}_{8}$ and MINER $v \mathrm{~A}$ data may sometimes look counterintuitive. So a contrasting of the standard and log-normal estimators would provide useful additional control.

Many data subsets (not necessarily the true CCQE ones) are reported in terms of reconstructed neutrino energy $E_{v}^{\mathrm{QE}}$ and square of the four-momentum transferred $Q_{\mathrm{QE}}^{2}$ for the simplest case when the target nucleon is at rest and there is no nuclear effects, except binding. These "quasielastic" variables are defined through the muon kinematics which can be reconstructed squarely and without reference to an interaction model:

$$
\begin{aligned}
E_{\nu}^{\mathrm{QE}} & =\frac{M^{\prime 2}-\left(M-E_{b}\right)^{2}-m_{\mu}^{2}+2\left(M-E_{b}\right) E_{\mu}}{2\left(M-E_{b}-E_{\mu}+P_{\mu} \cos \theta_{\mu}\right)}, \\
Q_{\mathrm{QE}}^{2} & =2 E_{v}^{\mathrm{QE}}\left(E_{\mu}-P_{\mu} \cos \theta_{\mu}\right) .
\end{aligned}
$$

Here $m_{\mu}, M$, and $M^{\prime}$ are the masses of muon, incoming, and outgoing nucleon, respectively; $E_{b}$ is the initial-state nucleon's removal energy (in fact an effective parameter tunable to reproduce the experimental data and thus in general different in different experiments, even those that work with targets of the same composition); $E_{\mu}, P_{\mu}$, and $\theta_{\mu}$ are, respectively, the total energy, absolute value of the momentum, and scattering angle of the outgoing muon. Normally, the quantity $E_{v}^{\mathrm{QE}}$ closely correlates with the true neutrino energy $E_{v}^{\text {True }}$ (see, e.g., Fig. 12 in Ref. [116]) and in any case the comparisons between the data and predictions expressed in terms of exactly the same well-defined variables do not introduce additional uncertainties or indeterminations.

The comparisons of the model predictions with selected recent data are presented in Figs. 12, 13, 14, 15, 16, 17, 18, 19, 20, 21, 22, 23, 24, 25 and 26 and in Tables 1, 2, 3, 4, 5, 6, 7,8 and 9 , which will be discussed in detail below in this section. The normalization factors, $\mathscr{N}$, shown in the Tables, are written with three digits in the mantissa. This certainly excessive precision is only needed in order to distinguish (usually small) differences between the two FSI models. Recall that the data of ND280 [25,144] and MINERvA [115,116], as well as an essential part of the MiniBooNE datasets [13,14] were not involved into the statistical analysis. These data are shown in the figures by open symbols.

\subsection{MiniBooNE}

In this section, we discuss a detailed comparison between the data from the Mini Booster Neutrino Experiment FNAL-E0898 (MiniBooNE) $[13,14]$ and respective predictions of the $\mathrm{SMRFG}+M_{A}^{\text {run }}$ model, two G1 8 tunes and besides (for the true CCQE scattering data only) of the SuSAv2-MEC [65], SuSAM* [68,200], and GIBUU [192].

The ultra-pure mineral oil filling the MiniBooNE detector medium is composed of $\mathrm{C}_{n} \mathrm{H}_{2 n+2}$ molecules with the length of the carbon chain $n=20-40(\sim 33$ on average, although the values differ in the literature). Since the cross sections only depend on the relative amount of each atomic species, in our simulations with GENIE 3 , the $v / \bar{v}$ interaction is chosen to be an "average" single unit on the hydrocarbon chain $n\left(\mathrm{CH}_{2.06}\right)$. Hereafter, for brevity's sake, we will refer to the MiniBooNE oil as $\mathrm{CH}_{2}$. For the GIBUU and superscaling 
models under consideration, only the calculations for pure carbon target are currently available.

The MiniBooNE event sample was selected by requiring a single well-reconstructed muon, and no final-state pions. The published dataset $[13,14,201]$ consists of both CCQElike and CCQE-corrected cross sections. The former sample includes not only the FSI contributions but also complicated instrumental and methodical effects and the CCQE sample is cleared of it all; in particular, the contributions of single pion interactions in carbon is removed according to the ReinSehgal (RS) model [90] as it implemented into the NUANCE MC neutrino event generator used in the MiniBooNE analyses. The $\bar{v}_{\mu}$ dataset [14] includes the CCQE and CCQE-like samples on both mineral oil and on carbon by subtracting the $\bar{v}_{\mu}$ CCQE events on hydrogen.

\subsubsection{Treatment of CCQE-like backgrounds}

Although the CCQE-like measurements are certainly less model-dependent than the CCQE measurements, their reliable modeling is complicated by indirect and very detectordependent statistical analysis used in the MiniBooNE data processing for finding the CCQE-like backgrounds and by complex flavor composition of the NuMI beam in the $v$ and $\bar{v}$ modes (see Fig. 8) and these complications are highly interconnected. The CCQE-like background in the $v$ mode is dominated by single positive pions. The procedure for selecting the CCQE sample and measuring the $\mathrm{CC} 1 \pi^{+}$background includes five steps [13]: (i) selection of events with a clean CC signature; (ii) separation of the selected CC sample into CCQE and $\mathrm{CC} 1 \pi^{+}$subsamples using a sequence of cuts; (iii) measurement of the $\mathrm{CC} 1 \pi^{+}$rate from the second subsample; (iv) adjustment of the RS model used in the NUANCE based $\mathrm{CC} 1 \pi^{+}$events simulation, to reproduce the measured $\mathrm{CC} 1 \pi^{+}$rate; and (v) subtraction of the adjusted $\mathrm{CC} 1 \pi^{+}$background (and other predicted backgrounds) from the CCQE signal to produce the CCQE cross section.

The background estimation for the $\bar{v}$ mode is more complicated. As is evident from Fig. 8, the $v_{\mu}$ contribution to the $\bar{v}$-mode flux is much more significant compared to the $\bar{v}_{\mu}$ component of the $v$-mode beam: the ratio of the integral $v_{\mu}$ and $\bar{v}_{\mu}$ fluxes in the $v$ mode is about 2.3 times larger than the inverse ratio in the $\bar{v}$ mode. This is mainly because of the leading-particle effect in hadronic interactions: protonberyllium collisions, forming the $v_{\mu}$ and $\bar{v}_{\mu}$ beams, preferentially produce about twice as many $\pi^{+}$as $\pi^{-}$[13]. Moreover, the overall contamination rate in MiniBooNE is much more significant in the $\bar{v}$-mode since the $v_{\mu}$ cross section is about thrice higher than the $\bar{v}_{\mu}$ ones in the $\sim 1 \mathrm{GeV}$ energy range. The NUANCE simulation predicts [13] that $\bar{v}_{\mu}$ events account for $\sim 1 \%$ of the $\nu$-mode event sample, while $v_{\mu}$ events are the cause of about $30 \%$ of the full $\bar{v}$-mode sample. The fraction of all non- $v_{\mu}$ events in the final CCQE event

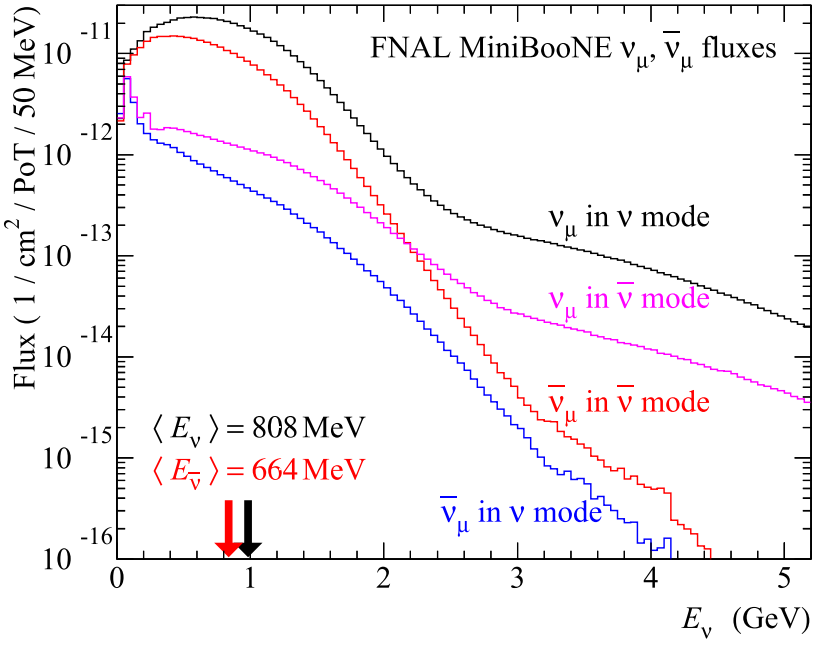

Fig. 8 Total $v_{\mu}$ and $\bar{v}_{\mu}$ flux predictions at the MiniBooNE detector with horn running in neutrino and antineutrino modes [202,203]

sample in the $v$ mode is $1.4 \%$ [13], while in the $\bar{v}$ mode, the contribution of all $v_{\mu}$ events to the $\bar{v}_{\mu}$ CCQE sample is about 22\% [204]. Three independent methods were used to constrain, tune, and subtract the $v_{\mu}$ contamination prediction [203].

The CCQE-like measurements exclude the $\bar{v} / v$ content of the subtracted data in the $v / \bar{v}$ mode. This, we repeat, is rather model- and detector-dependent procedure and it is difficult to reproduce this part of the MiniBooNE analysis in a third-party simulation. In order to avoid the unrealizable full reanalysis of the MiniBooNE data but without neglecting the above-mentioned features of the experiment, we use a simplified approach. Namely, we simulate the CCQE-like contributions in GENIE 3 by using the RS model for the pion production with no cut on the hadronic invariant mass, $W$, and with all the input parameters chosen as close as possible to those adopted in the NUANCE neutrino event generator used by the MiniBooNE experiment $[13,14]$. Then we form the ratios

CCQE-like contribution with GENIE setting CCQE-like contribution with NUANCE setting

for the cross sections of each type and for all model under consideration, and multiply it (bin by bin) to the corresponding full CCQE-like background contribution reported in Refs. [13,14]. This approach allows us to take into account all "instrumental" corrections and tunes, as well as the individual features of the investigated models for the inelastic interactions and FSI effects (see Sect. 4). Although the GENIE 3 generator cannot perfectly reproduce all the details of the hadronization model, models for shallow inelastic scattering (SIS) and other, less important but not negligi- 


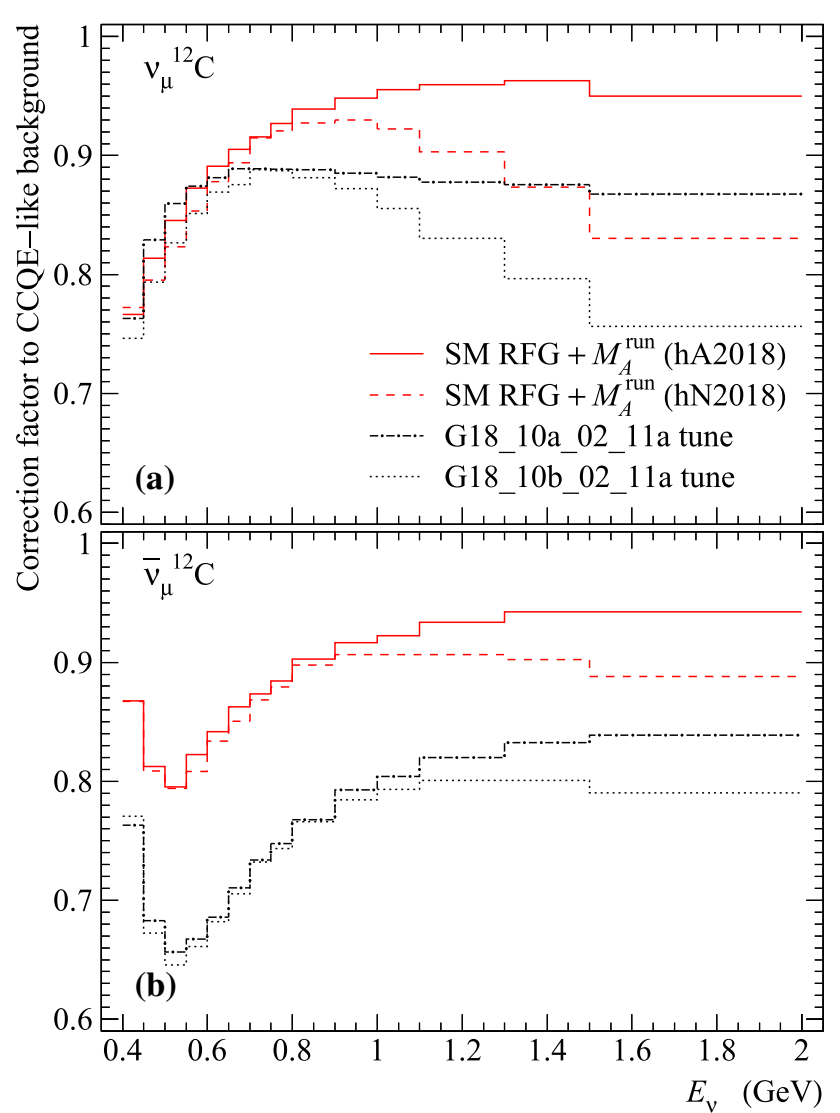

Fig. 9 The correction factors (14) for the $v_{\mu}{ }^{12} \mathrm{C}$ and $\bar{v}_{\mu}{ }^{12} \mathrm{C}$ total CCQE cross sections calculated using the settings for the $\mathrm{SMRFG}+M_{A}^{\text {run }}$ model with hA2018 and hN2018 FSI contributions, G18_10a_02_11a, and G18_10b_02_11a tunes

ble issues implemented into the NUANCE generator, ${ }^{3}$ the expected overall impact of the differences to the CCQE-like cross sections is insignificant and can be neglected in the $\chi^{2}$ tests discussed below.

Figures 9, 10, and 11 show the ratios (14) representing the correction factors to the full CCQE-like backgrounds for the total, single-differential, and double-differential CCQE cross sections, respectively. The 3D histograms in Fig. 11 are cropped at the top to avoid showing the unphysical peaks that occur near the kinematic boundaries (where the generated number of events is very small) due to statistical fluctuations in the Monte Carlo simulations.

It is seen that the correction factors for the backgrounds to the total CCQE cross sections are systematically less than 1 all our models (Fig. 9); at energies below $0.8-0.9 \mathrm{GeV}$ they slowly depend of the FSI model but at higher energies the differences become more essential. In case of the G18 model, the same is also true for the $\bar{v}_{\mu}{ }^{12} \mathrm{C}$ cross sections of all kinds.

\footnotetext{
${ }^{3}$ For example, in calculations of the denominator of Eq. (14), we use the hA2018 FSI model; GENIE 3 treatment of the resonance to DIS transition region is also different from what used in the NUANCE generator.
}

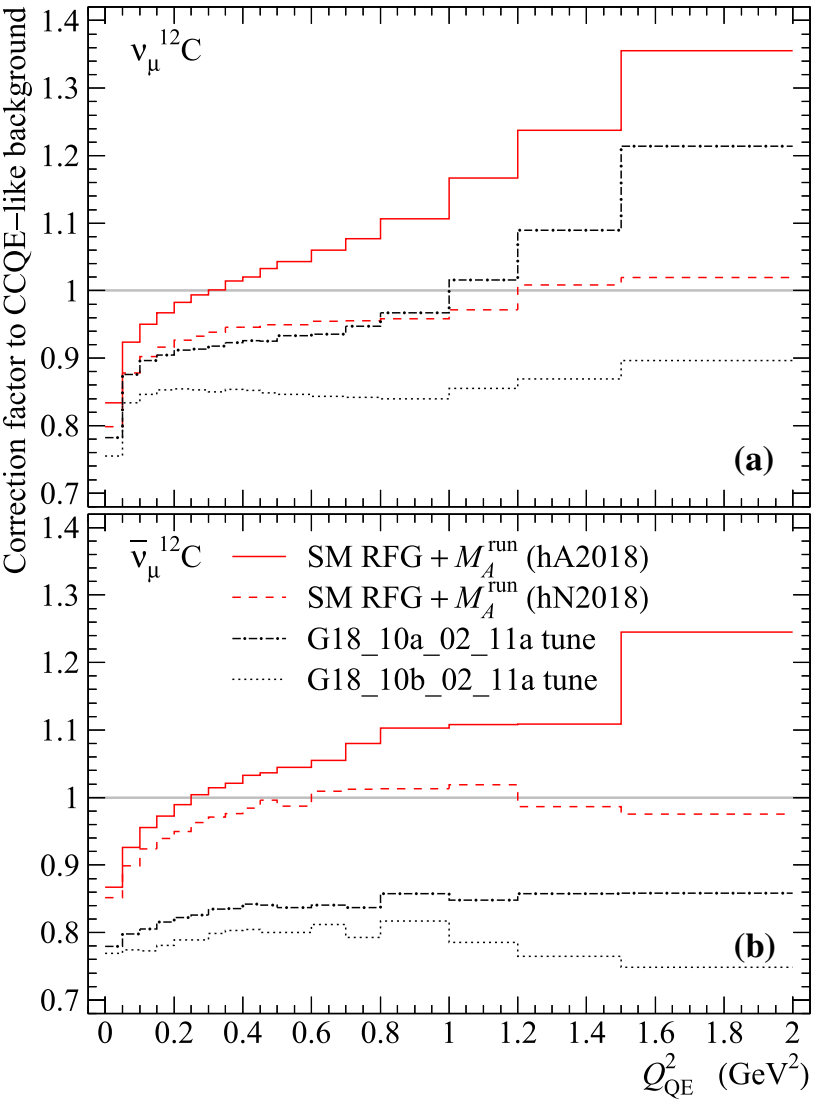

Fig. 10 The correction factors (14) for the $v_{\mu}{ }^{12} \mathrm{C}$ and $\bar{v}_{\mu}{ }^{12} \mathrm{C}$ differential CCQE cross sections, $d \sigma / d Q_{\mathrm{OE}}^{2}$ (see Fig. 14b, f) calculated using the settings for the SMRFG $+M_{A}^{\text {run }}$ model with hA 2018 and hN2018 FSI contributions, G18_10a_02_11a, and G18_10b_02_11a tunes

The correction factors for the single- and double-differential CCQE cross sections (see Figs. 10 and 11) either decrease or increase the CCQE-like backgrounds in comparison to the NUANCE predictions, depending on the kinematic region, but the regions where the correction factors are $\gtrsim 1$ make small contributions to the cross sections and hence, integrally the corrections reduce the MiniBooNE estimations of the CCQE-like backgrounds. Everywhere, the hA 2018 FSI model provides larger corrections then the $\mathrm{hN} 2018$ model. The $\mathrm{SMRFG}+M_{A}^{\text {run }}$ inelastic contributions are either similar to (at very low energies in the $v$ mode) or (everywhere in the $\bar{v}$ mode) larger than those estimated using the G18 inputs.

\subsubsection{RES to QES ratio}

As an illustration of the accuracy of the $\mathrm{CC} 1 \pi^{+}$background simulation, we present in Fig. 12 a comparison between the MiniBooNE data from Ref. [205] and GENIE 3 predictions (using the four models under discussion) for the ratio of the total $\mathrm{CC} 1 \pi^{+}$-like and $\mathrm{CCQE}$-like $v_{\mu} \mathrm{CH}_{2}$ cross sections. The ratios are plotted as functions of the true neutrino energy. This 

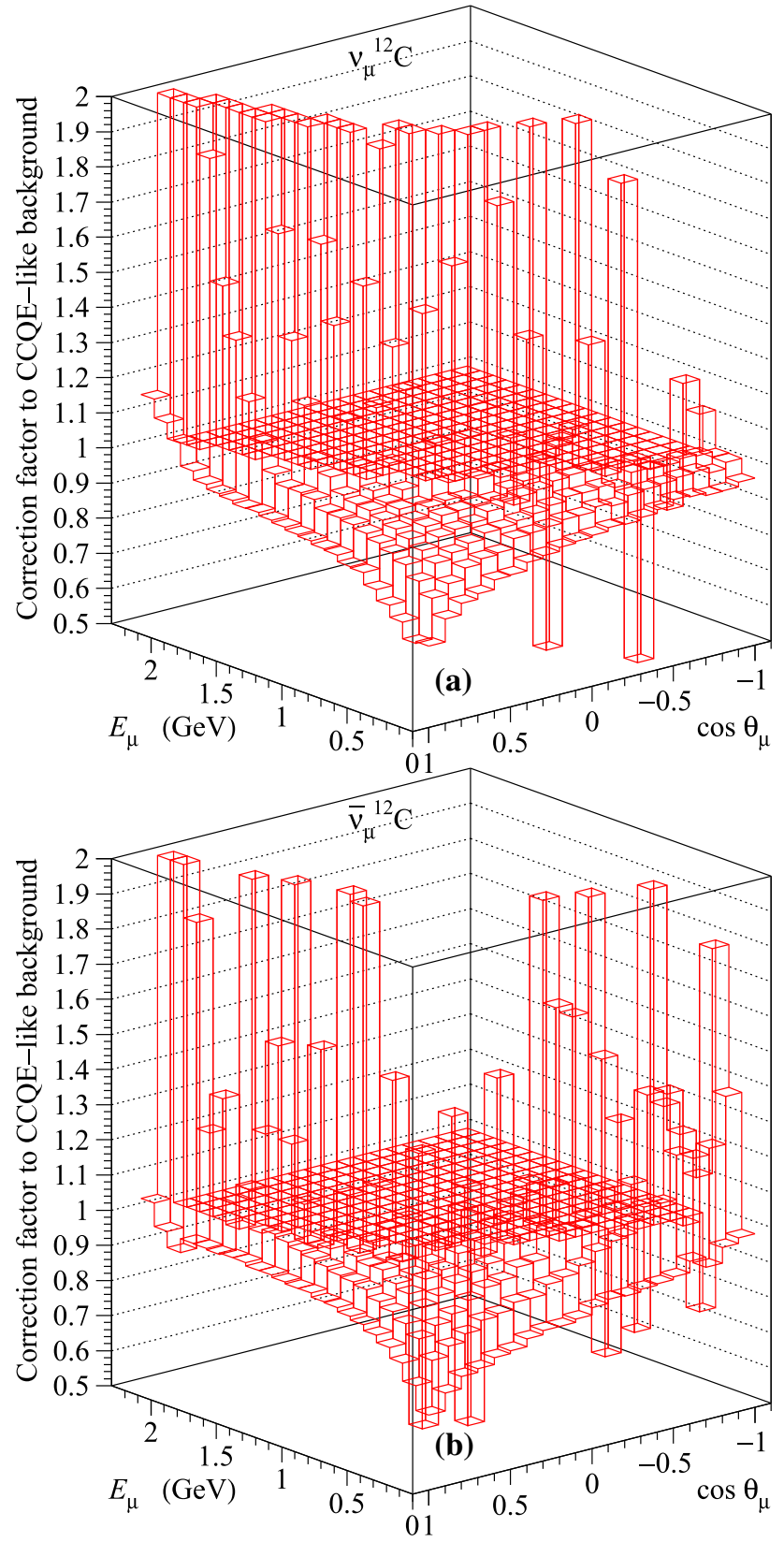

Fig. 11 The correction factors (14) for the $v_{\mu}{ }^{12} \mathrm{C}$ and $\bar{v}_{\mu}{ }^{12} \mathrm{C}$ doubledifferential CCQE cross sections, $d^{2} \sigma_{\nu} / d E_{\mu} d \cos \theta_{\mu}$ (see Figs. 15 and 16) calculated using the settings for the $\mathrm{SMRFG}+M_{A}^{\text {run }}$ model with hA 2018 FSI contributions

ratio is very convenient in that it is almost independent of the neutrino flux normalization and, to a lesser degree, contaminations. In all calculations with the $\mathrm{SM} R F G+M_{A}^{\text {run }}$ model, we used the updated KLN-BS singe-pion production model shortly described in Sect. 2. It is seen that the predicted ratios are slowly sensible to the FSI models and for both of them are in good agreement with the data $\left(\chi_{\text {st }}^{2} / \mathrm{ndf}=0.55\right.$ and 0.48 for hA 2018 and hN2018, respectively). Agreement with the $\mathrm{G} 18$ tunes is worse $\left(\chi_{\mathrm{st}}^{2} / \mathrm{ndf}=1.37\right.$ and 1.30

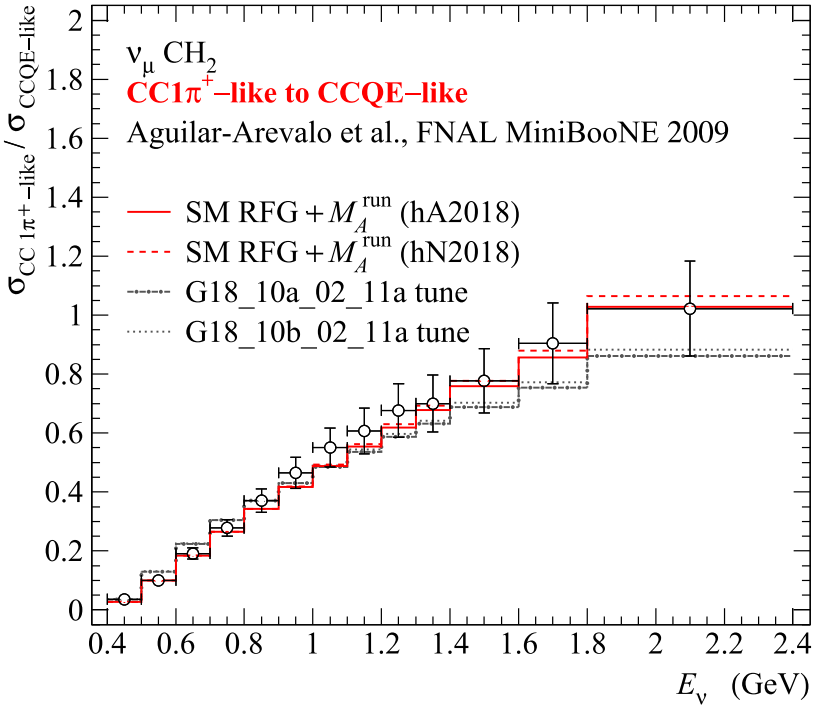

Fig. 12 Ratio of the total cross sections for CC singe $\pi^{+}$neutrinoproduction and CCQE-like neutrino scattering on mineral oil measured with MiniBooNE [205] vs. true neutrino energy. Vertical error bars include all sources of statistical and systematic uncertainty. Histograms show predictions of the $\mathrm{SMRFG}+M_{A}^{\text {run }}$ model and G18 tunes with the two versions of the FSI effect simulation

for G18_10a_02_11a and G18_10b_02_11a, respectively), but still quite acceptable. Let us remind that the GENIE 3 generator does not account for the interference between the resonances. We checked how this affects the ratio by applying a simple reweighting procedure and found that the interference effect is comparatively small but, unfortunately, slightly worsens the agreement of the SM RFG + $M_{A}^{\text {run }}$ model with the data; in terms of the standard $\chi^{2} \mathrm{~s}$ we got: $\chi_{\text {st }}^{2} /$ ndf $=0.85$ and 0.76 for, respectively, hA 2018 and $\mathrm{hN} 2018$ FSI. The G1 8 tunes are less sensitive to the interference among the resonance amplitudes: after accounting for this effect, we obtained $\chi_{\mathrm{st}}^{2} / \mathrm{ndf}=1.41$ and 1.33 for, respectively, G18_10a_02_11a and G18_10b_02_11a. The neutrino flux uncertainties are largely canceled in the ratio, and hence the good accord with the data provides an additional evidence in favor of the CCQE, $\mathrm{CC} 1 \pi^{+}$, and FSI models under consideration.

The MiniBooNE Collaboration also reported the $\mathrm{CC} 1 \pi^{+}$ to CCQE cross section ratio rescaled to an isoscalar target and the FSI corrected ratio. But the treatment of the FSI effects in Ref. [205] is so different from that in GENIE 3 that comparing these results with the $\mathrm{SMRFG}+M_{A}^{\text {run }}$ models is not very interesting. For completeness, we add that we also tested the KLN-BS model using the more recent MiniBooNE measurements of the total $\mathrm{CC} 1 \pi^{+}$cross section [206]. The comparison in particular demonstrates that (for both hA 2018 and hN 2018 FSI) the model slightly underestimates the data (with $\chi_{\text {st }}^{2} /$ ndf $=1.07$ ), but is in very good agreement with the cross-section shape in neutrino energy $\left(\chi_{\mathrm{st}}^{2} / \mathrm{ndf}=0.40\right)$. 


\subsubsection{Technical notes}

A few more specialized remarks are necessary:

(i) In our simulations, the (anti)neutrino flux averaging of the single- and double-differential cross sections is performed over the full energy range $[202,203]$ and thus our estimation of the mean $v_{\mu}$ and $\bar{v}_{\mu}$ beam energies (see legend in Fig. 8) is slightly different from those given in Refs. [13,14] for the energy range $0<E_{v, \bar{v}}<3 \mathrm{GeV}$. The spectral "tails" above $3 \mathrm{GeV}$ marginally affect the flux-folded CCQE cross sections but noticeably affect the CCQE-like background estimations, especially at high $Q^{2}$.

(ii) According to parameterization (2) the separation energy for carbon is $E_{b}^{\mathrm{C}} \approx 25 \mathrm{MeV}$. This value corresponds to one extracted from electron-nucleus scattering data [81] within the RFG model and hence we use it in all our simulations for the targets containing carbon. At the same time, for more accurate comparison of our calculations with the MiniBooNE data reported in terms of $E_{v}^{\mathrm{QE}}$ or $Q_{\mathrm{QE}}^{2}$, the value of $E_{b}^{C}$ in Eqs. (13) is set to $34 \mathrm{MeV}( \pm 9 \mathrm{MeV})-$ the value used in the MiniBooNE analyses.

(iii) The global fit of the running axial mass described in Sect. 3 is rather computer time consuming. To reduce the computing time, we used accurate analytical parametrizations for the $v_{\mu}$ and $\bar{v}_{\mu}$ energy spectra. The smooth (rational) parametrizations of the spectra, as opposed to the steplike dependences, significantly facilitate on-the-fly numerical integration. In contrast to this, in all our calculations with GENIE 3, we used the energy spectra presented as histograms. The difference between the total cross sections computed using the analytical parametrizations and histogrammic representation of the spectra is about $1 \%$. This is one of the reasons why the normalization factors obtained for the flux-averaged CCQE double-differential cross sections presented in Tables 1 and 2 and those listed in Appendix Table 13 are somewhat different.

Another reason of the marginal $(\lesssim 1 \%)$ differences is that in the global and individual fits, from which the normalization factors appeared in Appendix Table 13 were extracted, we used the CCQE data, which were singled out by the experimental methods (+ NUANCE modelling), while in the calculations with GENIE 3, we dealt with the true CCQE events. In other words, the definitions of the CCQE events are not fully identical in the MiniBooNE analysis and in the GENIE 3 simulation, due to different treatments of the FSI effects.

(iv) Estimations of the $\chi^{2}$ values and normalization factors $\mathscr{N}$ may generally depend on the choice of the normalization uncertainties, $\delta$, which cannot always be unambiguously fixed. We tested however that for all models under consideration, the $\chi_{\mathscr{N}}^{2}$ values decrease very slowly as $\delta$ increases and the factors $\mathscr{N}$ become almost independent of it as $\delta \gtrsim 0.1$. Therefore possible indetermination of $\delta$ does not significantly influence the subsequent comparisons and conclusions.

(v) Since the NUANCE generator takes into account the interference between the amplitudes of the resonances with the same spin and orbital momentum (within the RS model), it turns out (thanks to the procedure described above) to be automatically accounted for in our estimates of the CCQElike backgrounds.

(vi) For transforming the $\bar{v}_{\mu} \mathrm{CH}_{2}$ cross sections to the $\bar{v}_{\mu}{ }^{12} \mathrm{C}$ ones, the MiniBooNE analysis assumes an effective axial mass of $1.02 \mathrm{GeV}$ for the quasi-free hydrogen scattering component. The $21 \mathrm{MeV}$ uncertainty is applied according to Refs. [5,33]. We did not try to correct the hydrogen component subtraction by accounting our best-fit value of $M_{A}$ and other inputs, since the expected effect is very small in comparison with the above-mentioned uncertainties.

\subsubsection{Total CCQE and CCQE-like cross sections}

Figure 13 shows the ratios of the predicted and measured total cross sections for $v_{\mu}$ CCQE and CCQE-like scattering on carbon and, for $\bar{v}_{\mu}, \mathrm{CCQE}$ and CCQE-like scattering on carbon and MiniBooNE's mineral oil. The histograms representing the predictions of the $\mathrm{SMRFG}+M_{A}^{\text {run }}$ (with hA2018 FSI), G18_10b_02_11a SuSAv2-MEC [65], and SuSAM $^{*}$ [193] are plotted as functions of $E_{v}^{\mathrm{QE}, \mathrm{RFG}}$, the $v_{\mu} / \bar{v}_{\mu}$ energy reconstructed using the SM RFG model. In all calculations, we ignore minor (although sometimes not entirely insignificant) differences in the reconstructed energies. due to differences between the RFG parameters adopted in the MiniBooNE NUANCE and GENIE 3 input settings. The meaning of the shaded bands is explained in the legend and caption to the figure. With reference to Fig. 13 and Tables 1, 2 and 3, it can be concluded that the SM RFG + $M_{A}^{\text {run }}$ and the two superscaling models under consideration satisfactory describe both the absolute CCQE cross sections and $v_{\mu} / \bar{v}_{\mu}$ energy shapes well within the shape errors.

The SuSAv2-MEC model requires small or no renormalization $\left(|\mathscr{N}-1| \approx 0.03\right.$ for $v_{\mu}$ and $\approx 0.3$ for $\left.\bar{v}_{\mu}\right)$. The agreement of the model with the CCQE-like $v_{\mu}{ }^{12} \mathrm{C}, \bar{v}_{\mu} \mathrm{CH}_{2}$, and $\bar{v}_{\mu}{ }^{12} \mathrm{C}$ data is exceptionally good below $\sim 1 \mathrm{GeV}$, the predictions only slightly deviate from the mean measured values of the cross sections (always being within the shape errors) at higher energies. The renormalization is also ever not needed or inessential $(|\mathscr{N}-1| \ll \delta)$.

The G18 tunes predictions are close to the data (and to those of the SMRFG $+M_{A}^{\text {run }}$ model) at $E_{v}^{\mathrm{QE}, \mathrm{RFG}} \gtrsim 1 \mathrm{GeV}$ but at lower energies, they exhibit poorer consistency with the MiniBooNE CCQE and, to a greater degree, with the CCQE-like $v_{\mu}{ }^{12} \mathrm{C}, \bar{v}_{\mu} \mathrm{CH}_{2}$, and $\bar{v}_{\mu}{ }^{12} \mathrm{C}$ data. The agreement can be significantly improved by a renormalization, but with $\varrho \equiv|\mathscr{N}-1| / \delta \approx 1.2(0.9)$ and $\varrho=1.4-1.5$ (1.3) for, 


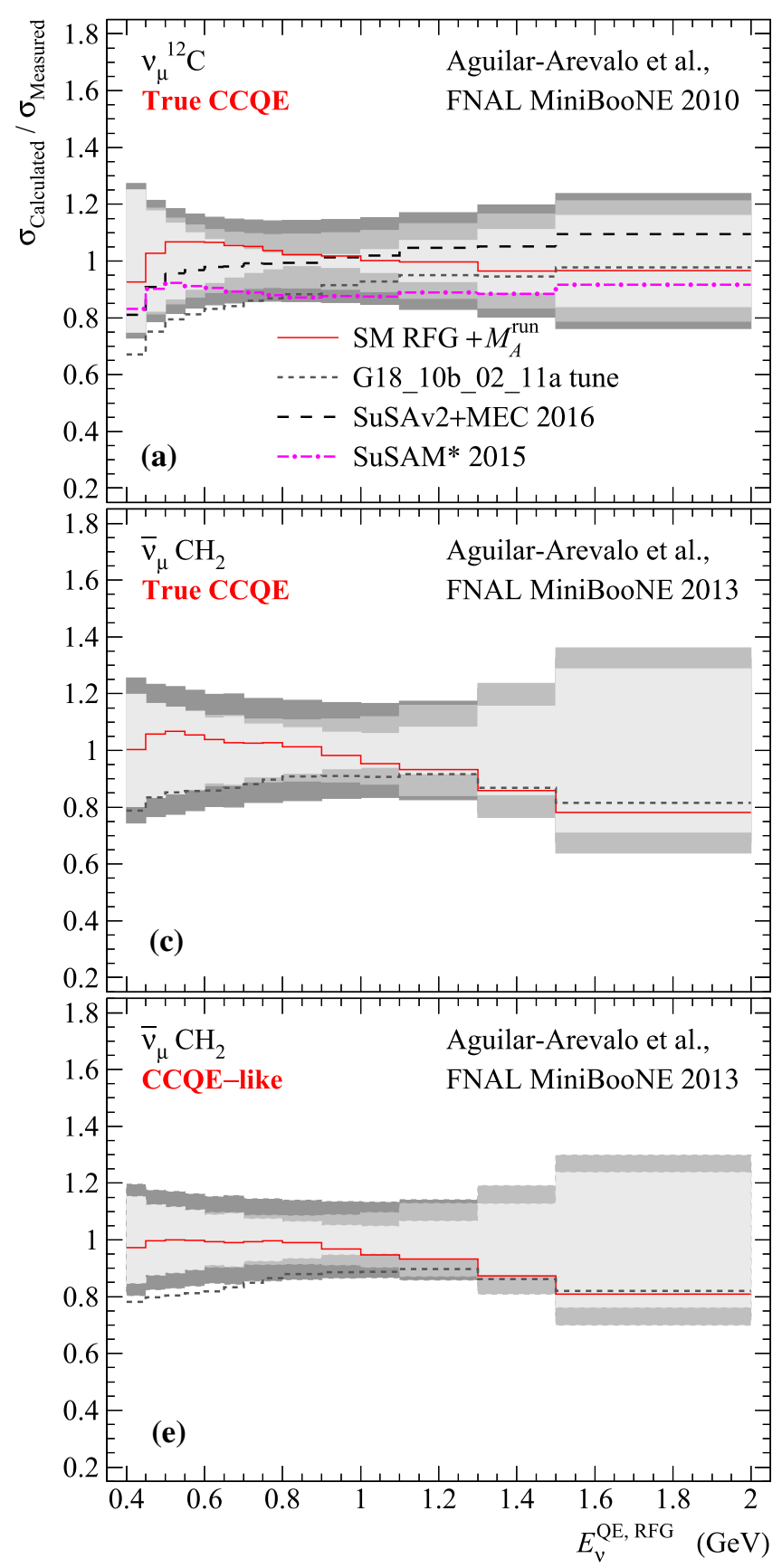

Fig. 13 Ratios of the total CCQE and CCQE-like cross sections measured by MiniBooNE $[13,14]$ to those predicted by several models. Shown are the ratios for the $v_{\mu}{ }^{12} \mathrm{C}(\mathbf{a}, \mathbf{b}), \bar{v}_{\mu} \mathrm{CH}_{2}(\mathbf{c}, \mathbf{e})$, and $\bar{v}_{\mu}{ }^{12} \mathrm{C}$ $(\mathbf{d}, \mathbf{f})$ cross sections; all are plotted as functions of "quasielastic" (anti)neutrino energy, $E_{v}^{\mathrm{QE}, \mathrm{RFG}}$, reconstructed using the RFG model. The light gray, gray and deep gray bands indicate, respectively, the shape

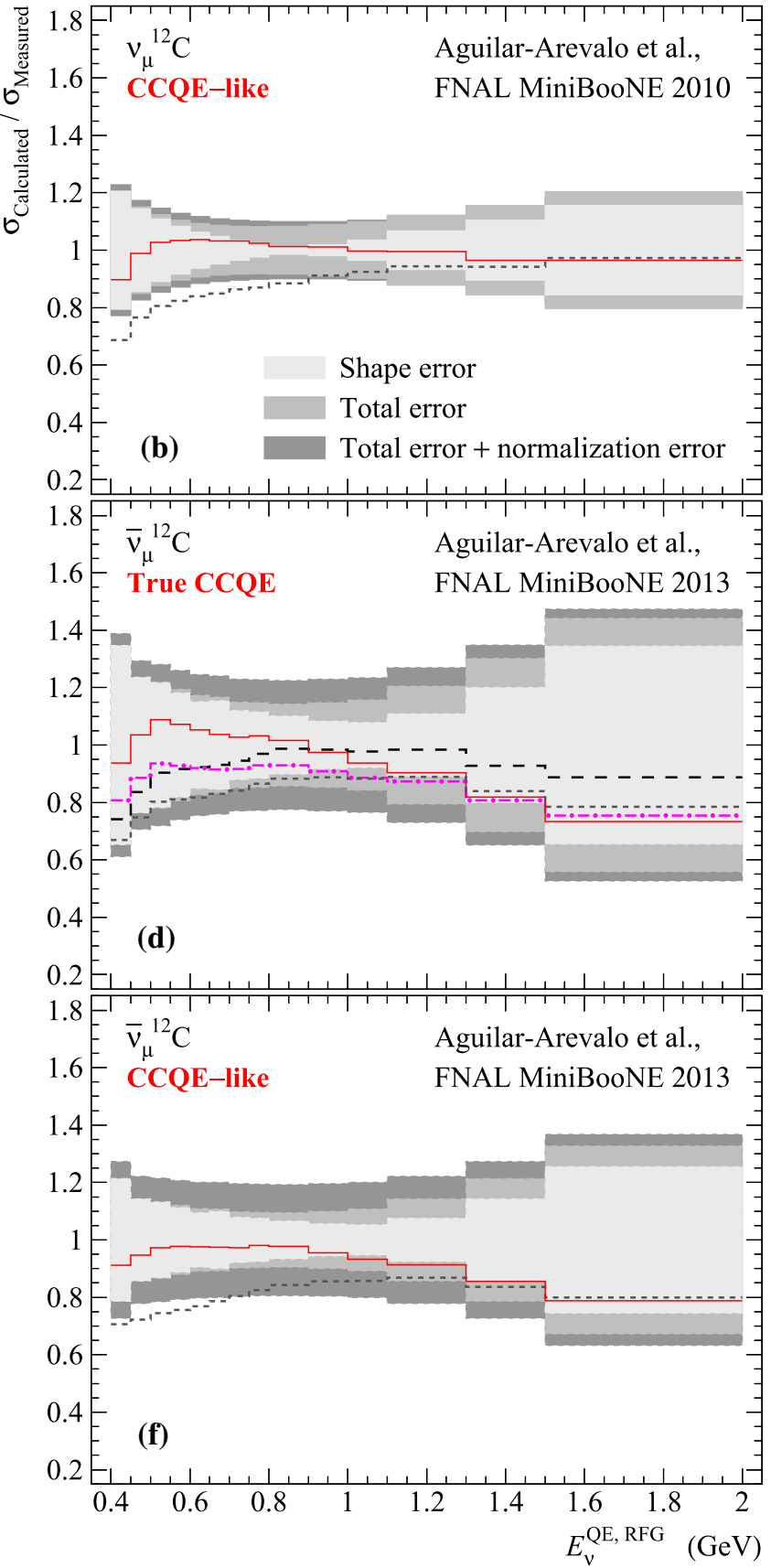

errors, total (statistical and systematic) errors except the normalization ones, and the full errors which include the normalization uncertainties whose values are given in Tables 1, 2 and 3). Histograms represent the predictions of SMRFG $+M_{A}^{\text {run }}$ (hA2018), G18_10b_02_11a tune, SuSAv2-MEC [65], and SuSAM* [193,200]. Quantitative comparison between the models and data is given in the mentioned tables 
respectively, CCQE $v_{\mu}\left(\bar{v}_{\mu}\right)$ and CCQE-like $v_{\mu}\left(\bar{v}_{\mu}\right)$ data samples. In other words, the G18 tunes well describe the shapes but not the absolute values of the cross sections. The difference in the FSI models does not essentially disturb the predictions.

The relevant predictions of the two superscaling models are only available for the CCQE samples on carbon. It is seen that both models are in good agreement with the data. The agreement with the SuSAM* model can be further improved by the renormalization with $\varrho \approx 1.2$ and 0.5 for, respectively, $v_{\mu}$ and $\bar{v}_{\mu}$ data samples (see Tables 1 and 2).

It is instructive to compare our calculations with the total $v_{\mu}{ }^{12} \mathrm{C} \rightarrow \mu^{-} X$ cross section measured by MiniBooNE [165] at precisely fixed $v_{\mu}$ energy of $236 \mathrm{MeV},-$ the world's-first known-energy, weak-interaction-only probe of the neutrino-nucleus interaction. The reported cross section is

$\sigma_{\text {tot }}^{\exp }=(2.7 \pm 0.9 \pm 0.8) \times 10^{-39} \mathrm{~cm}^{2} /$ neutron,

where the first error represents the total "rate+shape" uncertainty and the second comes from the uncertainty on the initial $K^{+} \rightarrow \mu^{+} v_{\mu}$ decay-at-rest neutrino flux. This result should be compared with

$$
\frac{\sigma_{\text {tot }}^{\mathrm{MC}}}{10^{-39} \mathrm{~cm}^{2} / \text { neutron }}=\left\{\begin{array}{l}
2.07\left(\mathrm{SuSAM}^{*}\right), \\
2.05\left(\mathrm{SMRFG}+M_{A}^{\mathrm{run}}\right), \\
1.95(\mathrm{SuSAv} 2-\mathrm{MEC}), \\
1.62(\mathrm{G} 18 \text { tunes }), \\
1.30 \text { (NuWRO), }
\end{array}\right.
$$

where the prediction of the NUWRO neutrino event generator $[207,208]$ is borrowed from Ref. [165]. The theoretical predictions are very weakly sensitive to the FSI model since the neutrino energy is below the inelastic threshold. The monoenergetic point is included into the joint MiniBooNE neutrino CCQE and CCQE-like datasets presented in Table 1. A visual comparison with several model predictions is shown in Fig. 28 (see Appendix B.1).

\subsubsection{Single differential cross sections}

Figure 14 shows a comparison of the measured and calculated flux-folded CCQE and CCQE-like differential cross sections, $d \sigma / d Q_{\mathrm{QE}}^{2}$, on carbon (for the $v_{\mu}$ case) and CCQE and CCQE-like cross sections on carbon and mineral oil (for the $\bar{v}_{\mu}$ case). The calculations were done in several models discussed above. The quantitative comparison is given in Tables 1, 2 and 3. To clarify the picture, the figure also shows (shaded histograms in panels (b), (e), and (f)) the CCQE-like background contributions estimated with the NUANCE MC neutrino event generator which is used by the MiniBooNE Collaboration for reconstructing the CCQE cross sections from the CCQE-like datasets $[13,14]$. These backgrounds are compared against those are re-weighted with the factors (14) calculated with GENIE 3 using the SM RFG $+M_{A}^{\text {run }}$ model setting, as described in Sect. 2.2, and hA 2018 FSI (long-dashed histograms); the correction factors for the four models are shown in Fig. 10 for the $v_{\mu}{ }^{12} \mathrm{C}$ and $\bar{v}_{\mu}{ }^{12} \mathrm{C}$ scatterings.

One can recognize that the NUANCE and GENIE 3 simulated background contributions are noticeably different in both shape and magnitude. The main reason of the differences is in the input parameters for pion production simulations, different descriptions of the SIS region and FSI models used in the two neutrino event generators. However, the differences themselves are relatively small in magnitude compared to the main contributions to the CCQE-like cross sections and thus the $\mathrm{SMRFG}+M_{A}^{\text {run }}$ model well reproduces both CCQE and CCQE-like cross sections, adequately simulating the "beyond RFG" nuclear effects.

It is seen from Fig. 14 and Tables 1, 2 and 3 that the SM $\mathrm{RFG}+M_{A}^{\text {run }}$ model is in full accord with the $v_{\mu}$ and $\bar{v}_{\mu}$ CCQE and CCQE-like data within the full errors (all values of $\chi_{\mathrm{st}}^{2} / \mathrm{ndf}$ are small) and almost no renormalization is needed ( $\varrho<0.2$ for all cross sections). Moreover, it is in agreement with almost all the data within the errors which do not include the normalization uncertainty. It is remarkably, that the GENIE 3 corrections to the CCQE-like backgrounds (see Fig. 10) work in the right direction, i.e. they improve agreement with the CCQE-like data. At the low- $Q^{2}$ region, the effect is mainly due to a decrease in the single-pion production cross sections caused by account of the muon mass in the KLN-BS model; and at high $Q^{2} \mathrm{~s}$, it is due to a cut-off in $W$, which increases the DIS contribution in the SIS region.

The G18 tunes substantially underestimate the CCQE and CCQE-like cross sections at low $Q^{2}$ s. The resulting $\chi_{1}^{2}$ values are incredibly large (see Tables 1,2 and 3) but it must be taken into account that the statistical errors in this dataset are notably small. In contrast to the SMRFG $+M_{A}^{\text {run }}$ model, the G18 corrections to the NUANCE simulated inelastic backgrounds either do not improve (for $v_{\mu}$ ) or even worsen (for $\bar{v}_{\mu}$ ) the agreement with the CCQE-like data, as a result of underestimating the inelastic contributions in the RES and SIS regions (see Sect. 4.2). The description of the data (for both $v_{\mu}$ and $\bar{v}_{\mu}$ ) can be improved but at the cost of large normalization factors: $\varrho \approx 1.9(1.0)$ and $\varrho=2.0-2.1$ $(1.1-1.2)$ for, respectively, CCQE $v_{\mu}\left(\bar{v}_{\mu}\right)$ and CCQE-like $v_{\mu}\left(\bar{v}_{\mu}\right)$ data samples. As for the SMRFG $+M_{A}^{\text {run }}$ model, the differences due to distinctions in the two GENIE 3 FSI models are expectedly small, although not entirely insignificant. For both SMRFG $+M_{A}^{\text {run }}$ model and G18 tunes, the agreement with the CCQE-like data on carbon is a little bit better than that on $\mathrm{CH}_{2}$, but this is due mainly to lesser systematic errors in the $\mathrm{CH}_{2}$ data sample, which is less model dependent.

The GiBUU model $(\mathscr{T}=1)[192]$ is in satisfactory agreement with the CCQE $v_{\mu}{ }^{12} \mathrm{C}$ and $\bar{v}_{\mu}{ }^{12} \mathrm{C}$ data at $Q_{\mathrm{QE}}^{2} \lesssim$ 


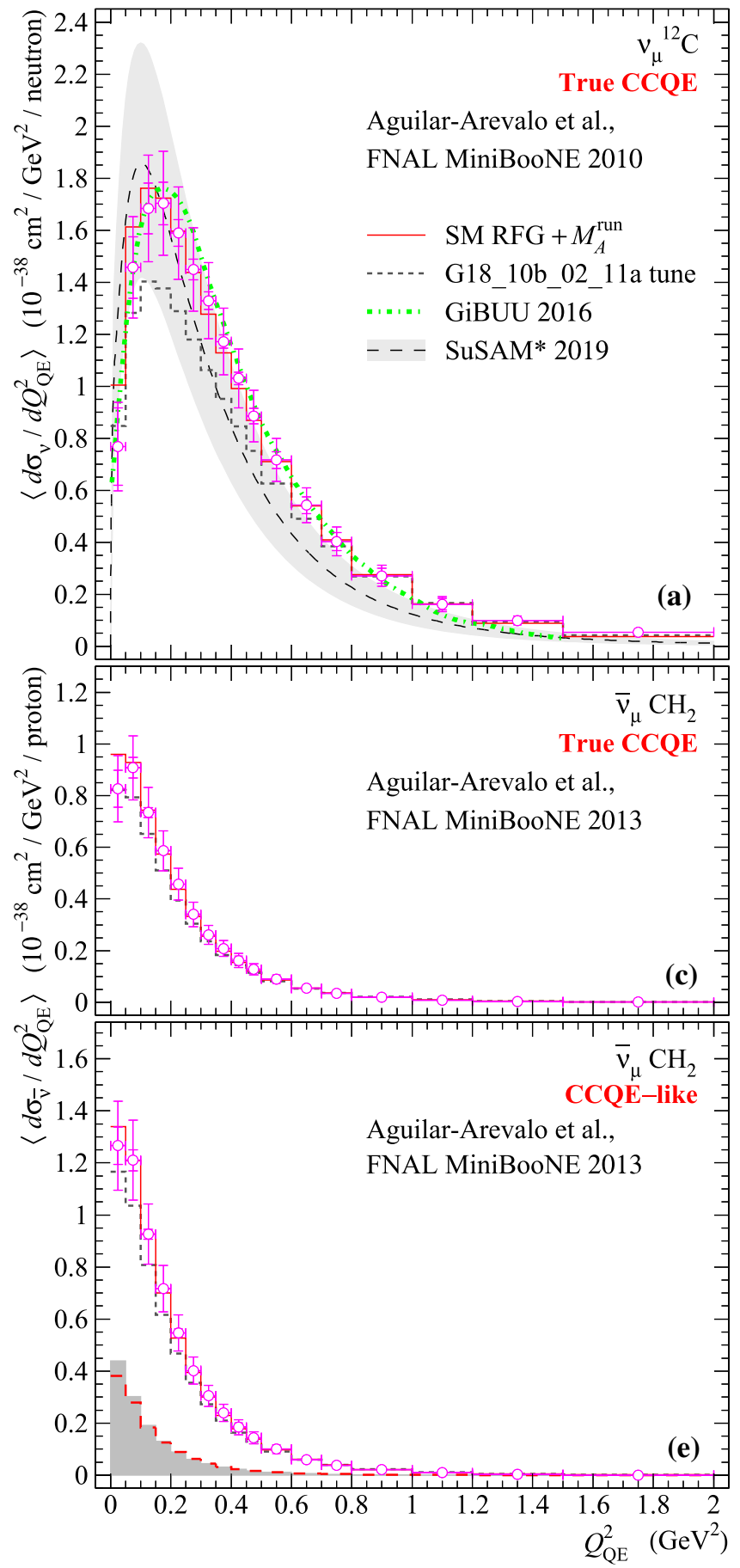

Fig. 14 Flux-weighted differential cross sections as functions of $Q_{\mathrm{OE}}^{2}$ for the true CCQE and CCQE-like neutrino and antineutrino scattering from mineral oil and carbon, as measured by the MiniBooNE detector $[13,14]$. The inner and outer vertical error bars indicate the total errors without and with the overall normalization uncertainties. The data were not involved into the global fit. The solid and dashed histograms represent predictions of the $\mathrm{SMRFG}+M_{A}^{\text {run }}$ model with hA2018 FSI contribution and G18_10b_02_11a tune. The dash-dotted curves in panels a and d show the results of the GIBUU model [192] and the

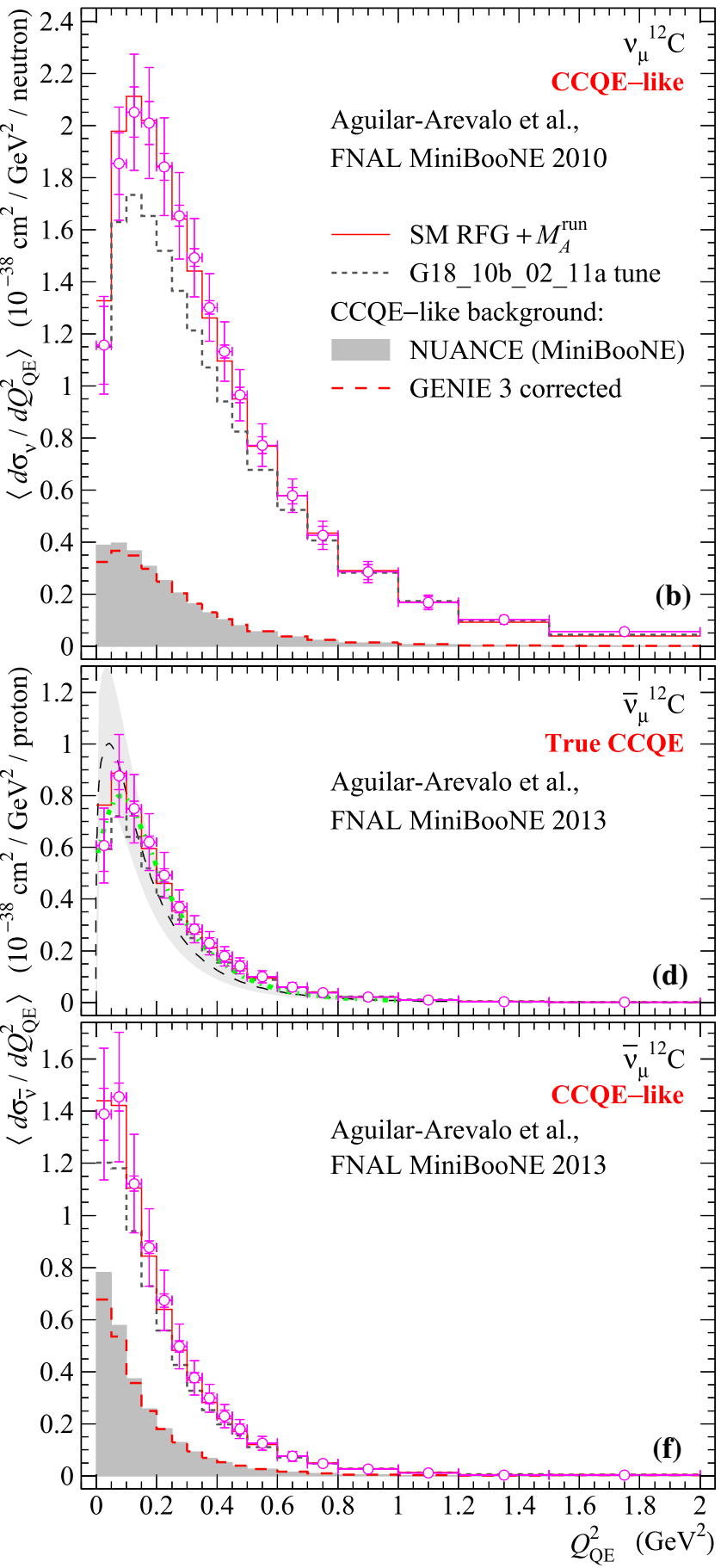

dashed curves are the results of the SuSAM* model $[68,200]$. The light gray bands around the dashed curves in panels $\mathbf{a}$ and $\mathbf{d}$ correspond to the uncertainties in the SuSAM* model input parameters. The shaded gray histograms in panels $\mathbf{b}$, e, and $\mathbf{f}$ represent the NUANCE simulated CCQE-like backgrounds as reported in Refs. [13,14]. The longdashed histograms in these panels represent the same backgrounds after applying the correction factors (14) computed with GENIE 3 using the $\mathrm{SMRFG}+M_{A}^{\text {run }}$ (hA 2018) model setting. Corresponding $\chi^{2} / \mathrm{ndf}$ values are listed in Tables 1, 2 and 3 
Table 3 Same as in Table 2 but for the CCQE-like $\bar{v}_{\mu} \mathrm{CH}_{2}$ and $\bar{v}_{\mu}{ }^{12} \mathrm{C}$ MiniBooNE data subsets [14]; respective numbers are shown before $\left(\mathrm{CH}_{2}\right)$ and after $\left({ }^{12} \mathrm{C}\right)$ slashes. The relevant data and selected model predictions are shown in Figs. 13e, f, 14e, f, and 17 . The normalization uncertainties, $\delta$, for each data subset are shown in parentheses

\begin{tabular}{|c|c|c|c|c|c|}
\hline Model & ndf & $\frac{\chi_{\mathrm{st}}^{2}}{\mathrm{ndf}}$ & $\frac{\chi_{1}^{2}}{\mathrm{ndf}}$ & $\frac{\chi_{\mathscr{N}}^{2}}{\text { ndf-1 }}$ & $\mathscr{N}$ \\
\hline \multicolumn{6}{|l|}{$\sigma_{\text {tot }}^{\mathrm{QE}}(\delta=12.4 \% / 16.9 \%)$} \\
\hline $\mathrm{SMRFG}+M_{A}^{\text {run }}(\mathrm{hA} 2018)$ & 14 & $0.07 / 0.09$ & $0.12 / 0.19$ & $0.09 / 0.08$ & $1.019 / 1.040$ \\
\hline $\mathrm{SMRFG}+M_{A}^{\text {run }}(\mathrm{hN} 2018)$ & 14 & $0.08 / 0.10$ & $0.13 / 0.21$ & $0.10 / 0.10$ & $1.021 / 1.044$ \\
\hline G18_10a_02_11a & 14 & $0.88 / 0.90$ & $2.11 / 2.70$ & $0.29 / 0.37$ & $1.164 / 1.223$ \\
\hline G18_10b_02_11a & 14 & $0.90 / 0.92$ & $2.14 / 2.76$ & $0.30 / 0.37$ & $1.166 / 1.227$ \\
\hline \multicolumn{6}{|c|}{$d \sigma / d Q_{\mathrm{QE}}^{2}(\delta=12.3 \% / 16.7 \%)$} \\
\hline $\mathrm{SMRFG}+M_{A}^{\text {run }}(\mathrm{hA} 2018)$ & 17 & $0.06 / 0.05$ & $0.32 / 0.40$ & $0.25 / 0.15$ & $1.013 / 1.028$ \\
\hline $\mathrm{SMRFG}+M_{A}^{\text {run }}(\mathrm{hN} 2018)$ & 17 & $0.06 / 0.06$ & $0.41 / 0.62$ & $0.26 / 0.16$ & $1.018 / 1.038$ \\
\hline G18_10a_02_11a & 17 & $0.62 / 0.51$ & $8.91 / 9.33$ & $0.52 / 0.40$ & $1.147 / 1.189$ \\
\hline G18_10b_02_11a & 17 & $0.66 / 0.56$ & $9.70 / 10.4$ & $0.56 / 0.43$ & $1.154 / 1.203$ \\
\hline \multicolumn{6}{|c|}{$d^{2} \sigma / d E_{\mu} d \cos \theta_{\mu}(\delta=12.4 \% / 16.7 \%)$} \\
\hline $\mathrm{SMRFG}+M_{A}^{\text {run }}(\mathrm{hA} 2018)$ & $78 / 75$ & $0.36 / 0.33$ & $0.52 / 0.56$ & $0.52 / 0.54$ & $1.003 / 1.011$ \\
\hline forward scattering & $70 / 67$ & $0.31 / 0.28$ & $0.48 / 0.50$ & $0.48 / 0.49$ & \\
\hline backward scattering & 8 & $0.75 / 0.77$ & $0.79 / 0.83$ & $0.80 / 0.88$ & \\
\hline $\mathrm{SMRFG}+M_{A}^{\text {run }}(\mathrm{hN} 2018)$ & $78 / 75$ & $0.36 / 0.34$ & $0.53 / 0.57$ & $0.53 / 0.55$ & $1.007 / 1.019$ \\
\hline forward scattering & $70 / 67$ & $0.31 / 0.28$ & $0.49 / 0.53$ & $0.48 / 0.49$ & \\
\hline backward scattering & 8 & $0.76 / 0.78$ & $0.80 / 0.84$ & $0.82 / 0.92$ & \\
\hline G18_10a_02_11a & $78 / 75$ & $0.55 / 0.50$ & $1.03 / 1.25$ & $0.48 / 0.38$ & $1.105 / 1.147$ \\
\hline forward scattering & $70 / 67$ & $0.55 / 0.50$ & $1.07 / 1.30$ & $0.45 / 0.36$ & \\
\hline backward scattering & 8 & $0.48 / 0.44$ & $0.52 / 0.52$ & $0.54 / 0.45$ & \\
\hline G18_10b_02_11a & $78 / 75$ & $0.57 / 0.53$ & $1.09 / 1.36$ & $0.49 / 0.39$ & $1.110 / 1.157$ \\
\hline forward scattering & $70 / 67$ & $0.57 / 0.54$ & $1.13 / 1.43$ & $0.47 / 0.37$ & \\
\hline backward scattering & 8 & $0.48 / 0.45$ & $0.52 / 0.52$ & $0.54 / 0.45$ & \\
\hline \multicolumn{6}{|c|}{ Joint dataset $\left(\delta_{\text {eff }}=12.3 \% / 16.8 \%\right)$} \\
\hline $\mathrm{SMRFG}+M_{A}^{\text {run }}(\mathrm{hA} 2018)$ & $109 / 106$ & & $0.43 / 0.47$ & $0.42 / 0.42$ & $1.010 / 1.023$ \\
\hline $\mathrm{SMRFG}+M_{A}^{\text {run }}(\mathrm{hN} 2018)$ & $109 / 106$ & & $0.46 / 0.52$ & $0.43 / 0.43$ & $1.015 / 1.032$ \\
\hline G18_10a_02_11a & $109 / 106$ & & $2.38 / 2.72$ & $0.48 / 0.40$ & $1.136 / 1.178$ \\
\hline G18_10b_02_11a & $109 / 106$ & & $2.55 / 2.98$ & $0.49 / 0.41$ & $1.142 / 1.189$ \\
\hline
\end{tabular}

$1 \mathrm{GeV}^{2}$ but underestimates the high- $Q^{2}$ tails of the differential cross sections. However a small renormalization $(-5.2 \%$ for $v_{\mu}$ and $+6.2 \%$ for $\bar{v}_{\mu}$ ) noticeably improves the agreement. At the same time, it should be pointed out that the authors of Ref. [192] do not provide calculations for the highest- $Q^{2}$ bins.

The SuSAM* model $[68,200]$ rather poorly describes the CCQE cross section shapes and this cannot be substantially corrected by a renormalization. In Fig. 14 we display the confidence bands, delineating the uncertainties arising due to variations in the input parameters of the SuSAM* model derived from the extensive global fit to electron scattering data [198]; in fact, the bands represent minimax over the $1 \sigma$ uncertainties of the parameters defining the form of the scaling function. Let us remind that the $\chi^{2}$ values listed in the tables do not take into account these uncertainties. Accounting for them will certainly improve the formal consistency with the data. We also recall that the model was not tuned to neutrino data.

\subsubsection{Double-differential cross sections}

Figure 15 shows the MiniBooNE $v_{\mu}$ data for the flux-folded CCQE and CCQE-like sections $d^{2} \sigma_{\nu} / d E_{\mu} d \cos \theta_{\mu}$ on carbon. Figures 16 and 17 show the MiniBooNE $\bar{v}_{\mu}$ [14] data for the flux-folded CCQE and CCQE-like double-differential cross sections on mineral oil and pure carbon. The cross sections are plotted as slices at fixed bins of $\cos \theta_{\mu}$ vs. $E_{\mu}$, where $\theta_{\mu}$ is the muon scattering angle and $E_{\mu}$ is the muon kinetic energy. In several panels of Figs. 16 and 17, the experimental data and relevant model predictions are rescaled for easier comparison of the cross section shapes displayed in the adjacent panels. Only the CCQE data shown in Figs. 15 and 16 were involved into the global fit of the running axial 

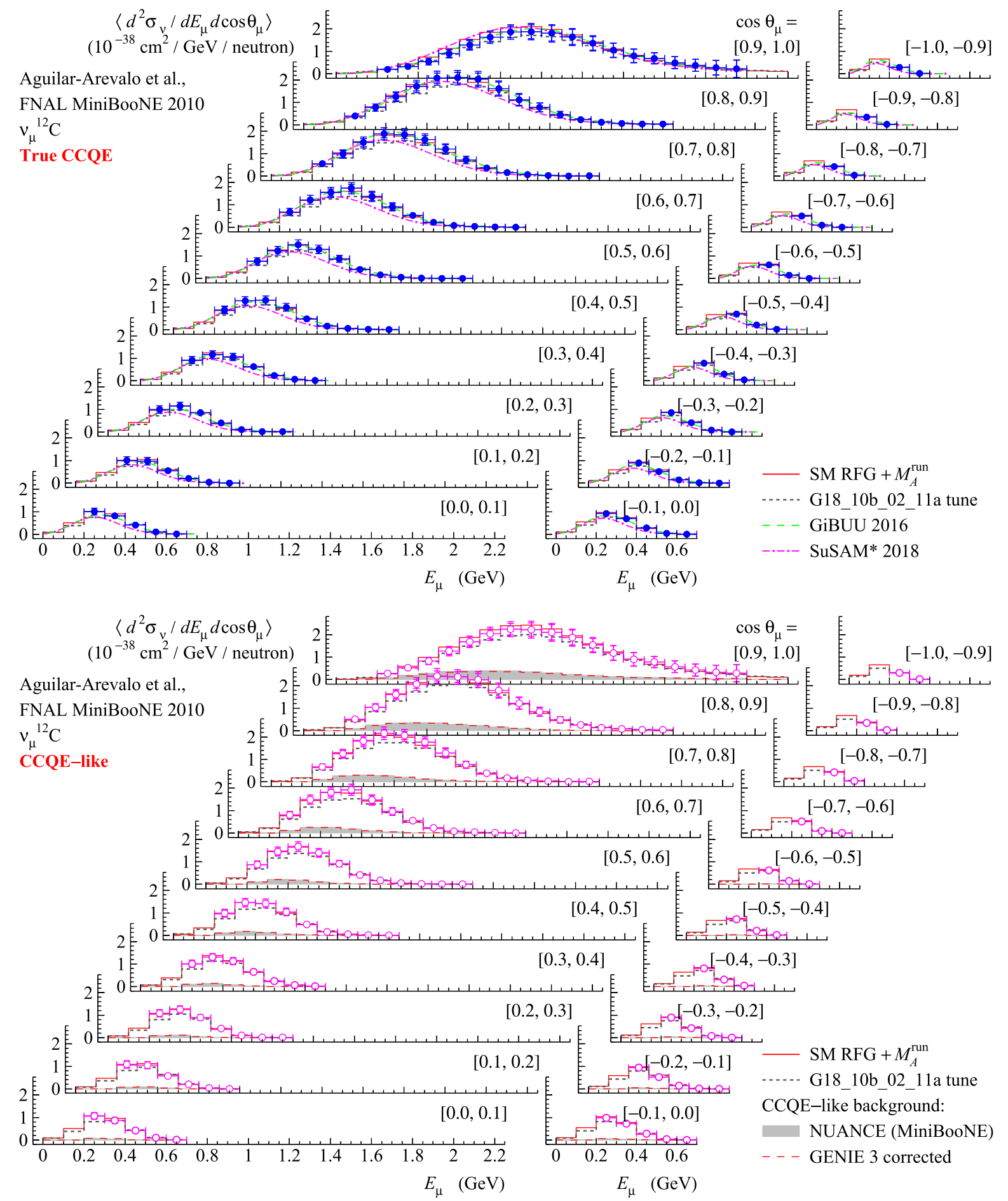

Fig. 15 Flux-weighted double-differential cross sections, $d^{2} \sigma_{\nu} / d E_{\mu} d \cos \theta_{\mu}$, for the true CCQE (twenty top panels) and CCQE-like (twenty bottom panels) $v_{\mu}$ scattering from carbon as measured with MiniBooNE [13]. The cross sections are displayed as functions of the muon kinetic energy, $E_{\mu}$, for several intervals of the cosine of the muon scattering angle, $\theta_{\mu}$ (shown in square brackets). The inner and outer vertical error bars indicate, respectively, the total errors without and with the normalization uncertainty $(\delta=10.7 \%$ for CCQE and $9.8 \%$ for CCQE-like data). Histograms represent predictions of the SMRFG $+M_{A}^{\text {run }}$ model (with hA2018 FSI) and G18_10b_02_11a tune. Smooth curves represent the GIBUU[192] and SuSAM* [68] model predictions. The shaded gray and long-dashed histograms show, respectively, the CCQE-like background contributions reported in Ref. [13] and the same after applying the correction factors (14). Corresponding $\chi^{2} /$ ndf values are listed in Table 1 

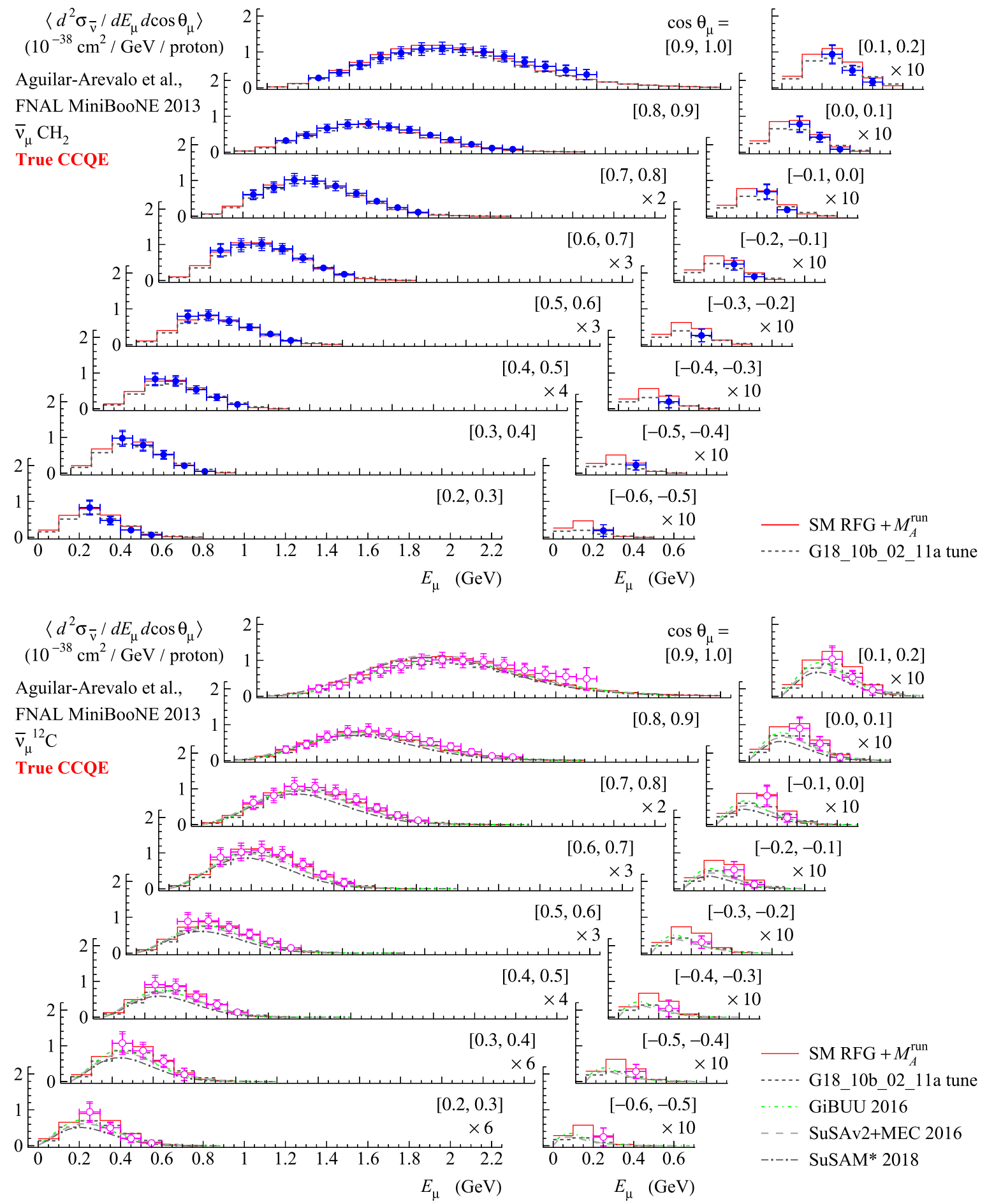

Fig. 16 Flux-weighted double-differential cross sections, $d^{2} \sigma_{\bar{v}}$ $/ d E_{\mu} d \cos \theta_{\mu}$, for the true CCQE $\bar{v}_{\mu}$ scattering from mineral oil (sixteen top panels) and pure carbon (sixteen bottom panels) as measured by MiniBooNE [14]. The notation is the same as in Fig. 15. The inner and outer vertical error bars indicate, respectively, the total errors without and with the normalization uncertainty $(\delta=13 \%$ for mineral oil and $17.2 \%$ for carbon). To aid the visualization, the data and histograms in some panels are multiplied by the factors indicated in the legends. The histograms represent predictions of the $\mathrm{SMRFG}+M_{A}^{\text {run }}$ model (with hA 2018 FSI) and G18_10b_02_11a tune. Smooth curves in bottom panels represent predictions of GIBUU[192] and of the two superscaling models - SuSAv2-MEC [65] and SuSAM* [68]. Corresponding $\chi^{2} /$ ndf values are listed in Table 2 


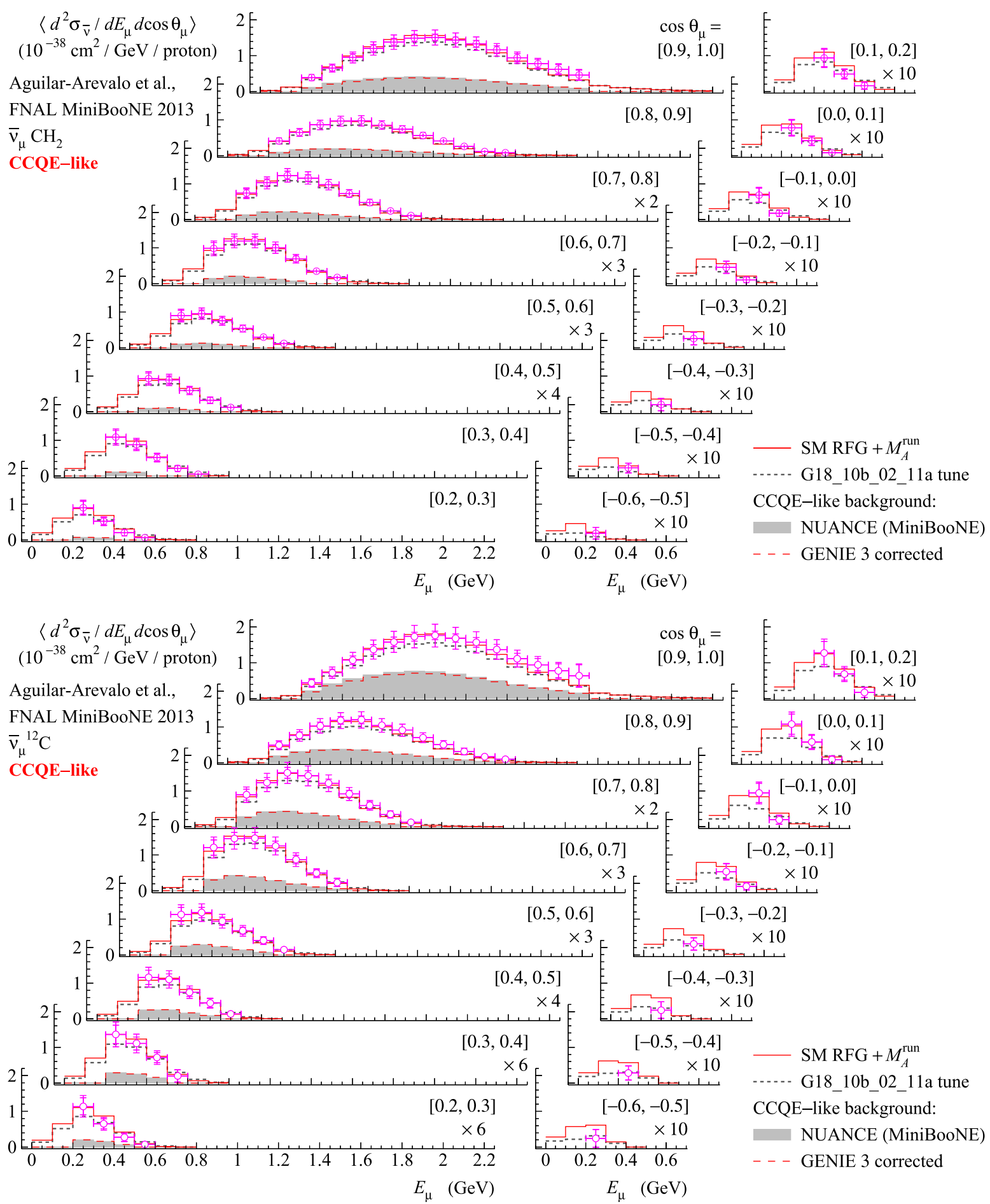

Fig. 17 Flux-weighted double-differential cross sections, $d^{2} \sigma_{\bar{v}} / d E_{\mu} d \cos \theta_{\mu}$, for the CCQE-like $\bar{v}_{\mu}$ scattering from mineral oil (sixteen top panels) and pure carbon (sixteen bottom panels) as measured by MiniBooNE [14]. The solid and dashed histograms represent predictions of the SMRFG $+M_{A}^{\text {run }}$ model (with hA 2018 FSI) and G18_10b_02_11a tune, respectively. The shaded gray and longdashed histograms represent, respectively, the CCQE-like background contributions reported in Ref. [14] and the same after applying the correction factors (14) calculated using the $\mathrm{SMRFG}+M_{A}^{\text {run }}$ (hA2018) model setting (see Fig. 11b). The inner and outer vertical error bars indicate, respectively, the total errors without and with the normalization uncertainty ( $\delta=12.4 \%$ for mineral oil and $16.7 \%$ for carbon). Other designations have the same meaning as in Fig. 16. Corresponding $\chi^{2} /$ ndf values are listed in Table 3 
mass. The data are compared with several model predictions; no normalization has been applied. In order not to overload the figures, not all models under consideration are presented in the figures. In particular, we display only one version of the FSI corrections for the SMRFG $+M_{A}^{\text {run }}$ model and G1 8 tune (the differences due to the two FSI versions are usually small).

In Figs. 15, 16, and 17, we show the CCQE-like backgrounds modeled with the NUANCE neutrino event generator $[13,14]$ (shaded histograms) and those corrected using GENIE 3 with the SMRFG $+M_{A}^{\text {run }}$ (hA 2018) model settings (long-dashed histograms); the backgrounds that are visually indistinguishable from zero are not displayed to avoid cluttering the figures. The corresponding correction factors are presented in Fig. 11. Tables 1, 2 and 3 include detailed lists of $\chi^{2}$ s for each model under examination, evaluated both for the entire kinematic range and for the forward and backward scattering subregions.

It can be seen from the figures and Tables 1, 2 and 3 that the $\mathrm{SMRFG}+M_{A}^{\text {run }}$ model provides very good agreement with the CCQE and CCQE-like data in all kinematic regions for both $v_{\mu}$ and $\bar{v}_{\mu}$ datasets. The model practically does not require renormalization $(\varrho \lesssim 0.2)$. The two G18 tunes also provide reasonable agreement with the data but slightly underestimate the measured cross sections in certain kinematic domains. Since there is no specific difficulties related to the forward/backward scattering, consistency with the data can partly be improved by an overall renormalization with $\varrho \approx 0.6-1.2$ which is still acceptable within the reported experimental normalization uncertainty.

The GIBUU and SuSAv2-MEC models both show good agreement with the CCQE $v_{\mu}{ }^{12} \mathrm{C}$ data for the essential part of the forward scattering hemisphere (see Fig. 15) and with the CCQE $\bar{v}_{\mu}{ }^{12} \mathrm{C}$ data in the whole kinematic range (see Fig. 17). In certain kinematic domains, the GIBUU $\chi^{2}$ values for the $\bar{v}_{\mu}{ }^{12} \mathrm{C}$ cross sections are similar to or even better than (after acceptable renormalization) those for the SM $\mathrm{RFG}+M_{A}^{\text {run }}$ model. However, both GIBUU and SuSAv2MEC exhibit difficulties in reproducing the $v_{\mu}{ }^{12} \mathrm{C}$ cross section shapes in the backward scattering hemisphere and an overall renormalization cannot resolve the conflict with the MiniBooNE data. The unexpectedly large $\chi^{2}$ values for the $\mathrm{CCQE} \bar{v}_{\mu}{ }^{12} \mathrm{C}$ double-differential cross section predicted by the GIBUU in comparison with, e.g., SuSAM* model (see Table 1) arise because of systematic bias of the GIBUU prediction from several data points at highest muon energies and at the backward scattering angles, visually indistinguishable in Fig. 15.

The SuSAM* model offers only a qualitative description of the CCQE cross section shapes (see Figs. 15 and 16), which cannot be substantially corrected by a renormalization (see Table 1). We must, however, remind that the SuSAM* model operates with a set of adjustable parameters which can be further tuned to improve accordance with the MiniBooNE data.

\subsubsection{Joint datasets}

The joint $v_{\mu}{ }^{12} \mathrm{C}, \bar{v}_{\mu} \mathrm{CH}_{2}$, and $\bar{v}_{\mu}{ }^{12} \mathrm{C}$ datasets presented at the bottom of Tables 1,2 and 3 include the MiniBooNE data on the CCQE and CCQE-like total, single-differential, and double-differential cross sections. The analysis shows that

(a) all six MiniBooNE data subsamples are well consistent with each other and

(b) the SMRFG $+M_{A}^{\text {run }}$ model very well describes all these subsamples almost irrespective of the FSI model.

It is essential that only a relatively small part of the full MiniBooNE dataset (true CCQE double-differential cross sections) has been involved into the fit of $M_{A}^{\text {run }}$. This shows good predictive power of the model within the MiniBooNE energy range. On the other hand, even this part of the MiniBooNE data has significant statistical weight in the full experimental dataset used in the global fit. This is due to large number of the data-points and relatively small total errors, excluding the normalization uncertainties, which, however, do not substantially affect the above conclusions since the $\mathrm{SMRFG}+M_{A}^{\text {run }}$ model requires almost no normalization to the data. Thus, the MiniBooNE dataset critically influences the fitting parameters $M_{0}$ and, even to a greater extent, $E_{0}$. The G1 8 tunes reasonably describe the MiniBooNE cross section shapes but claims essential renormalization (sometimes larger than the data normalization uncertainty) in order to fit the absolute values of the cross sections.

\subsection{T2K ND280}

In this section, we discuss a comparison of the SM RFG + $M_{A}^{\text {run }}$ model and several others theoretical predictions with the recent data on pionless interactions from T2K's off-axis fully magnetized ND280 near detector on the J-PARC site [209], obtained using two detector targets: water based [144] and one composed of plastic scintillator $\left(\mathrm{C}_{8} \mathrm{H}_{8}\right)$ and metal [25]. In our calculations, the cross sections predicted for both T2K ND280 experiments are averaged over the updated $v_{\mu}$ flux [210] in the full simulated neutrino energy range to about $30 \mathrm{GeV}$. While the narrow energy spectrum of the beam is centered around $600 \mathrm{MeV}$ and has the mean energy of about $870 \mathrm{MeV}$, the high-energy tail marginally $(\lesssim 1 \%)$ contributes to the inelastic backgrounds.

\subsubsection{Water target}

The T2K ND280 experiment with the $\mathrm{H}_{2} \mathrm{O}$ target selected the CCQE-like events without pions in the final state (so-called 
"CCO $\pi$ " events). It is expected that more than one nucleons may be ejected out of the nucleus due to intranuclear and multinucleon interactions. The number of post-FSI nucleons, however, are not fully controlled in the experiment. Our simulations reproduce all essential features of the experiment, in particularly, the $\mathrm{CC} 0 \pi$ events with any number of secondary nucleons are considered as the signal.

Figure 18 shows a comparison of the model predictions with the flux-folded double-differential cross section for the CCQE-like $v_{\mu}$ scattering from the water target. Table 4 collects the values of $\chi_{\text {st }}^{2} /$ ndf, $\chi_{1}^{2} /$ ndf, $\chi_{\mathscr{N}}^{2} /($ ndf -1$)$, and of the normalization factor, $\mathscr{N}$. These values are obtained using the detailed contributions to the covariance matrix from all sources of uncertainties, provided by the authors. The normalization uncertainty, $\delta$, is taken to be $8.76 \%$. It is seen that all the models under consideration are broadly consistent with the data. Formally, the best agreement occurs for the two G1 8 tunes; they as well require the least renormalization $(\varrho \approx 3.9 \%)$. The FSI effects simulated with the hA 2018 and hN2018 models are practically indistinguishable. The $\chi^{2}$ values for the $\mathrm{SMRFG}+M_{A}^{\text {run }}$ model (also very weakly dependent of the FSI effect modeling versions) are worse but entirely satisfactory and can be somewhat improved by an essential renormalization $(\varrho \approx 1.3 \%)$. The cross sections predicted by the SuSAM* 2018 model are taken from Ref. [68]. The model does not take into account the FSI effects and this is, probably, one of the reasons of considerably high $\chi^{2}$ s. We emphasize that Ref. [68] does not provide predictions for the muon momenta above $3 \mathrm{GeV}$. So, the statistical analysis for this model is made with the reduced dataset.

The last column in Table 4 shows the ratios of the measured and predicted flux-averaged total cross sections $\sigma=$ $\sigma_{v_{\mu} \mathrm{H}_{2} \mathrm{O}}^{\mathrm{CCO} \pi}$ in the restricted region of the phase space. The experimental result of Ref. [144] is

$$
\begin{aligned}
\sigma_{\exp }= & \left(0.95 \pm 0.08_{\text {stat }} \pm 0.06_{\text {detector syst }}\right. \\
& \left. \pm 0.04_{\text {model syst }} \pm 0.08_{\text {flux }}\right) \times 10^{-38} \mathrm{~cm}^{2} / \text { neutron }
\end{aligned}
$$

All the models under consideration predict essentially lower cross sections, namely

$\sigma_{\mathrm{GENIE}}=0.79(0.66) \times 10^{-38} \mathrm{~cm}^{2} /$ neutron

for $\mathrm{SMRFG}+M_{A}^{\text {run }}$ (G18); the predictions of these models are almost insensitive to the FSI model. A rough estimation made with the SuSAM* 2018 model yields

$\sigma_{\text {SuSAM }^{*}} \simeq 0.64 \times 10^{-38} \mathrm{~cm}^{2} /$ neutron

For comparison, the NEUT 5.4.0 neutrino generator (default in the present $\mathrm{T} 2 \mathrm{~K}$ analyses) predicts [144]

$\sigma_{\mathrm{NEUT}}=0.66 \times 10^{-38} \mathrm{~cm}^{2} /$ neutron (the same as for the G18 tunes). According to Ref. [144], the tension is primarily (but not only) due to discrepancies between the data and MC simulations in the large-angle regions, which cover an essential part of the reduced phase space. This is, at least in part, likewise true for other models under examination and thus indicates some disagreements between the T2K and MiniBooNE measurements.

\subsubsection{Hydrocarbon target}

Figure 19 shows the flux-weighted CCQE-like doubledifferential cross sections of three types, plotted as functions of the leptonic variables, proton momentum, $P_{p}$, and cosine of the proton scattering angle, $\cos \theta_{p}$; the data are from the same experimental sample as shown in Fig. 20. We transformed the original data presented by the authors of experiment as the single-differential cross sections $d \sigma_{\nu} / d P_{\mu}$, $d \sigma_{\nu} / d P_{p}$, and $d \sigma_{\nu} / d \cos \theta_{p}$ to the double-differential ones; this is done for the convenience of comparing the data with each other and with similar data from MINERvA and MiniBooNE. For similar reasons, the cross sections calculated as function of $P_{\mu}$ at lowest values of $\left|\cos \theta_{\mu}\right|$ are multiplied by the scale factors shown in the legends.

The T2K ND280 experiment with the hydrocarbon target also selected $\mathrm{CC} 0 \pi$ events, but classified them by number of final state protons with momenta above $500 \mathrm{MeV} / c$. The distribution of these events, $d \sigma_{\nu} / d N_{p}$, in the number of secondary protons, $N_{p}$, is shown in the bottom panel of Fig. 20. It is seen that the number of the final-state protons is in agreement with the $\mathrm{SMRFG}+M_{A}^{\text {run }}$ model prediction for $N_{p}=0$ and $N_{p} \geq 2$, but for the case $N_{p}=1$ the G18_10a_02_11a tune works better. The figure, as well, shows (see top panel) the differential CCQE-like cross section $d \sigma_{\nu} / d \cos \theta_{\mu}$ integrated over the muon momentum, $P_{\mu}$. The SM RFG $+M_{A}^{\text {run }}$ model with hA 2018 FSI and G1 8 tune predict similar shapes (both are in conformity with the data), but somewhat different absolute values. Looking at this plot, it can hardly be said that one model works better than the other in describing the muon angular distribution.

Let us now consider the comparison of the T2K data with predictions of the $\mathrm{SMRFG}+M_{A}^{\text {run }}$ (with hA 2018 FSI) model, G18_10b_02_11a tune, and GIBUU 2017 ( $\mathscr{T}=0)$ [211]. A visual comparison of the measured and predicted cross sections shows that the agreement between all model predictions and the data is generally unsatisfactory. Considering that this experiment (in contrast to the T2K experiment with water target and similarly to the MINER $v$ A experiment discussed below) does not provide the covariance matrix responsible for the flux uncertainties, one cannot unambiguously define the matrix $\widetilde{\mathbf{W}}$ (see Eq. (9)) and thus it is difficult to properly define the normalization factors without loss of information on the bin-by-bin correlations. We mention in passing that the $\chi^{2} \mathrm{~s}$ are very slowly sensitive to the global normalization 


\section{$\left\langle d^{2} \sigma_{v^{\prime}} / d P_{\mu} d \cos \theta_{\mu}\right\rangle$ \\ $\left(10^{-38} \mathrm{~cm}^{2} / \mathrm{GeV} /\right.$ neutron $)$}

Abe et al.,

T2K ND280 2018

$\mathrm{v}_{\mu} \mathrm{H}_{2} \mathrm{O}$

CCQE-like

\section{E}

E $\cos \theta_{\mu}=$

$[0.975,1.000]$

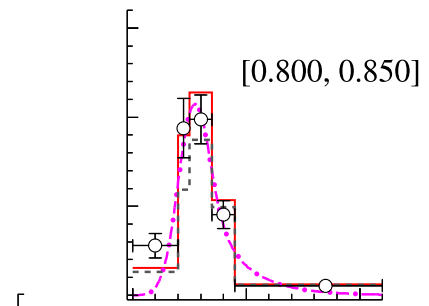

$[0.925,0.975]$

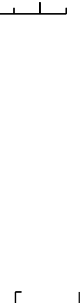

$[0.900,0.925]$

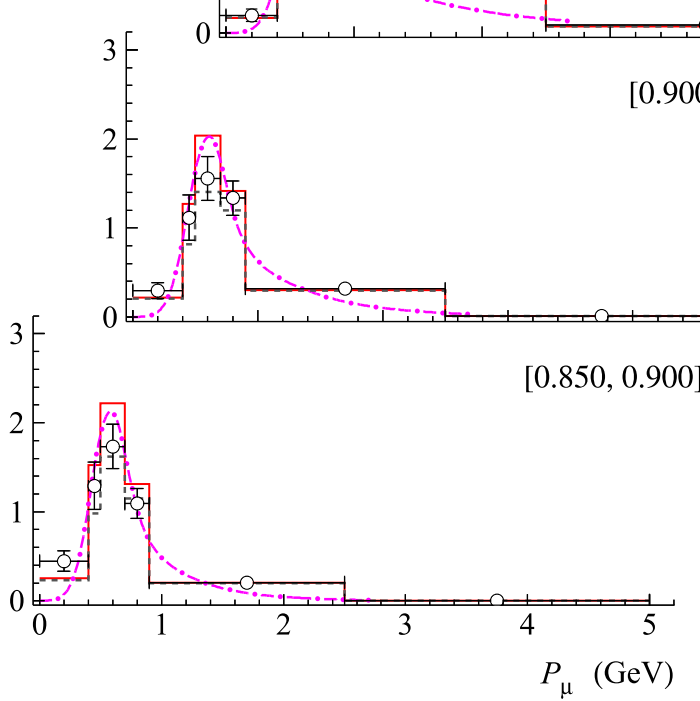

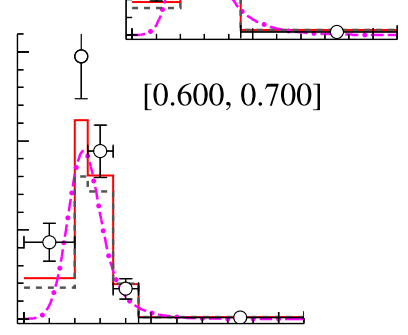

$[0.000,0.600]$

$[0.600,0.700]$
$[0.700,0.800]$
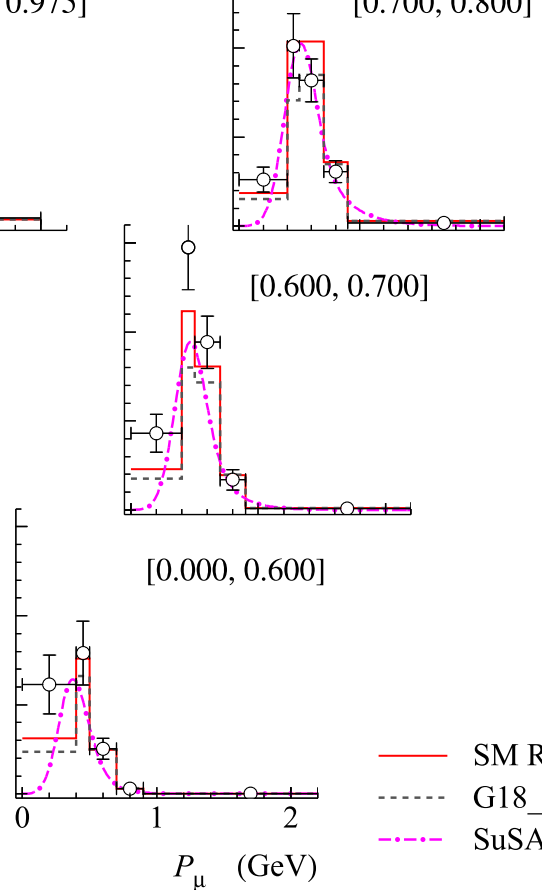

Fig. 18 Flux-weighted double-differential cross sections for the CCQE-like $v_{\mu}$ scattering from water target as measured by T2K ND280 [144] and plotted vs. muon momentum, $P_{\mu}$, for several intervals $\cos \theta_{\mu}$ (shown in square brackets). The vertical error bars represent the total errors including the normalization uncertainty of $8.76 \%$. Histograms represent the SMRFG $+M_{A}^{\text {run }}$ model and G18_10b_02_11a tune, curves show prediction of the SuSAM* 2018 model [68] obtained with no account for the FSI effects. For more details, see Table 4
Table 4 The values of $\chi_{\text {st }}^{2} /$ ndf, $\chi_{1}^{2} /$ ndf, $\chi_{\mathscr{N}}^{2} /($ ndf -1$)$, and $\mathscr{N}$, calculated for the CCQE-like double-differential cross section $d^{2} \sigma / d P_{\mu} d \cos \theta_{\mu}$, for neutrino scattering from water as measured by T2K ND280 [144] (see Fig. 18). The last column shows the ratios of the measured and predicted reduced flux-averaged total cross section $\sigma=\sigma_{v_{\mu} \mathrm{H}_{2} \mathrm{O}}^{\mathrm{CC} 0 \pi}$. All calculations are performed with four GENIE 3 models and by using the full covariance matrix with ndf $=45$. Result of the SuSAM* $^{*} 2018$ model is borrowed from Ref. [68]; the calculation was made without taking into account the FSI effects and for incomplete dataset $($ ndf $=40)$

\begin{tabular}{|c|c|c|c|c|c|c|}
\hline Model & $\frac{\chi_{\mathrm{st}}^{2}}{\mathrm{ndf}}$ & $\frac{\chi_{1}^{2}}{\mathrm{ndf}}$ & $\frac{\chi_{\mathscr{N}}^{2}}{\text { ndf-1 }}$ & $\mathscr{N}$ & $\frac{\chi_{\log }^{2}}{\mathrm{ndf}}$ & $\frac{\sigma_{\exp }}{\sigma_{\mathrm{MC}}}$ \\
\hline $\mathrm{SMRFG}+M_{A}^{\mathrm{run}}(\mathrm{hA} 2018)$ & 1.26 & 1.28 & 1.14 & 0.888 & 1.32 & $1.20 \pm 0.17$ \\
\hline $\mathrm{SMRFG}+M_{A}^{\mathrm{run}}(\mathrm{hN} 2018)$ & 1.29 & 1.30 & 1.16 & 0.888 & 1.32 & $1.20 \pm 0.17$ \\
\hline G18_10a_02_11a & 0.85 & 1.01 & 1.02 & 1.033 & 1.31 & $1.41 \pm 0.20$ \\
\hline G18_10b_02_11a & 0.85 & 1.01 & 1.02 & 1.034 & 1.31 & $1.41 \pm 0.20$ \\
\hline SuSAM* 2018 (no FSI) & 1.56 & 1.89 & 1.89 & 0.941 & 1.92 & $1.48 \pm 0.21$ \\
\hline
\end{tabular}



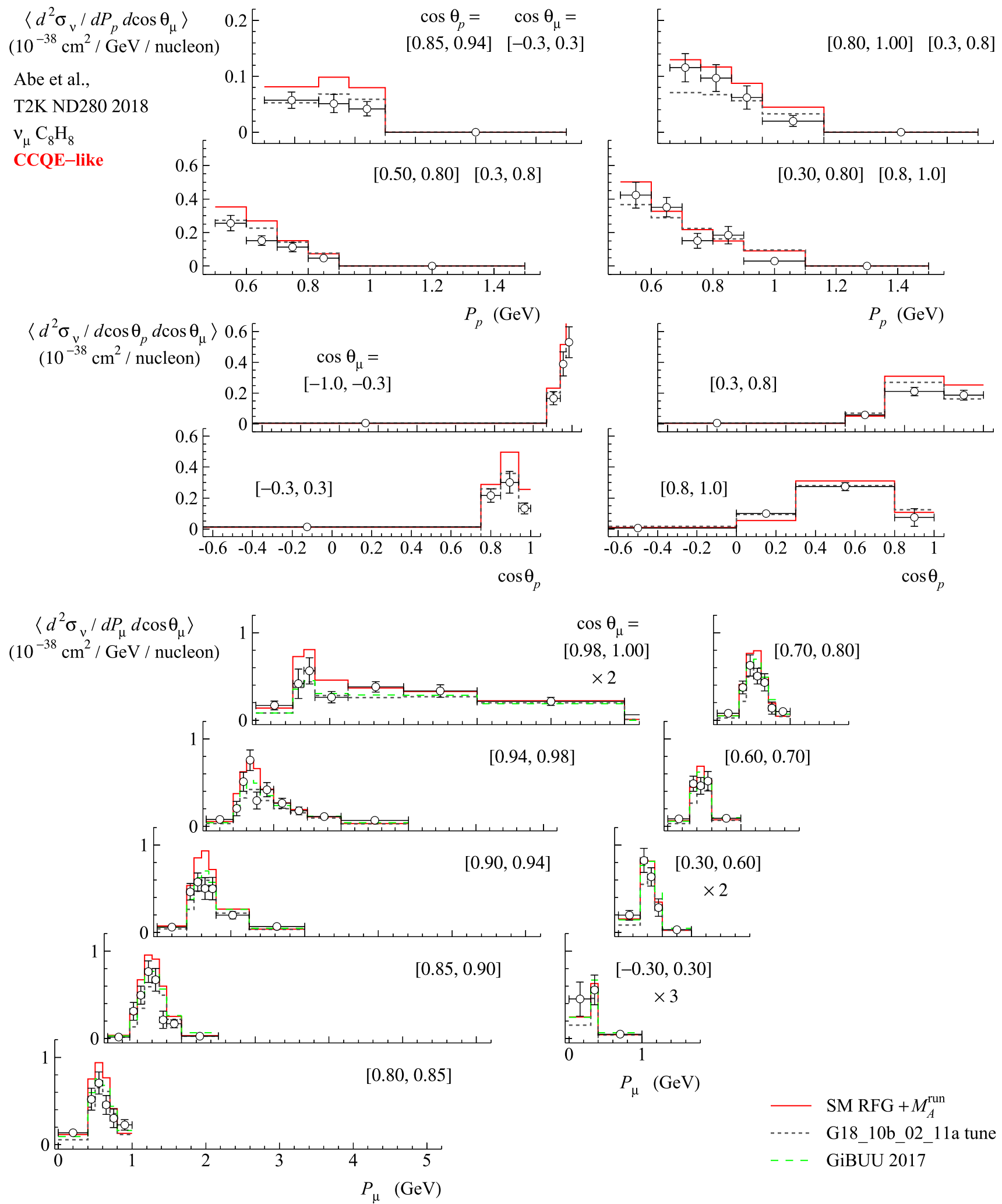

Fig. 19 Flux-weighted double-differential cross sections for the CCQE-like $v_{\mu}$ scattering from hydrocarbon target as measured by T2K ND280 [25] and plotted as function of proton momentum, $P_{p}$, for several intervals of the cosines of the proton and muon scattering angles, $\theta_{p}$ and $\theta_{\mu}$ (four top panels); as function of $\cos \theta_{p}$ for several intervals of $\cos \theta_{\mu}$ (four middle panels); and as function of muon momentum,
$P_{\mu}$, for several intervals of $\cos \theta_{\mu}$ (nine bottom panels). All intervals of fixed angular variables are shown in square brackets. The vertical error bars represent the total errors including the normalization uncertainty of $8.5 \%$. Histograms represent the SMRFG $+M_{A}^{\text {run }}$ (hA 2018) model, G18_10b_02_11a tune, and GIBUU $2017(\mathscr{T}=0)$ [211]. For more details, see Table 5 


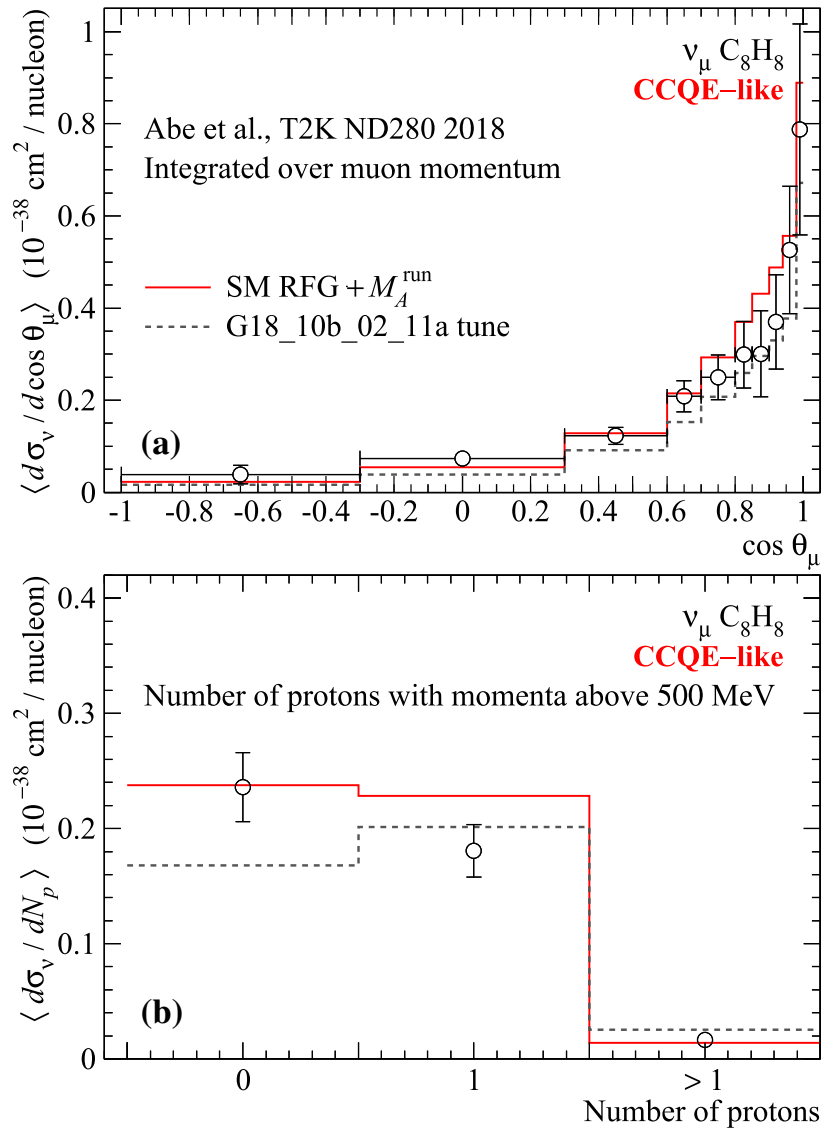

Fig. 20 Differential cross section vs. $\cos \theta_{\mu}$ integrated over $P_{\mu}$ (a) and distribution in the number of secondary protons with momenta $>500 \mathrm{MeV} / c$ (b). The vertical error bars represent the total errors including the normalization uncertainty. The T2K ND280 data points are from Ref. [25] $\left(\mathrm{C}_{8} \mathrm{H}_{8}\right)$. Histograms represent the $\mathrm{SMRFG}+M_{A}^{\text {run }}$ (hA2018) model and G18_10b_02_11a tune. Following the prescription of the T2K Collaboration, only the left bin in panel a and the right bin in panel $\mathbf{b}$ are included into the dataset used in calculations of $\chi^{2}$ s listed in Table 5

uncertainty. So to quantify the comparison more definitely, here and in the succeeding discussion, we use both the standard (8) and log-normal (10) least-squares criteria.

Table 5 collects the values of $\chi_{\text {st }}^{2} /$ ndf and $\chi_{\log }^{2} /$ ndf estimated for the full $\mathrm{T} 2 \mathrm{~K}$ dataset and for the subset containing only the differential cross sections measured in terms of the leptonic variables. Similar quantities estimated for the cross sections $d \sigma_{v} / d N_{p}$ are listed in Table 6. It is seen from the Tables that none of the models, including the default one from the T2K-tuned MC neutrino event generator NEUT 5.4.0 [212,213] (whose predictions are not shown in Fig. 20), can accurately describe the $\mathrm{T} 2 \mathrm{~K} \mathrm{C}_{8} \mathrm{H}_{8}$ data. It should be recorded here that NEUT 5.4.0 uses similar theoretical models for CCQE, $2 p 2 h$, resonance pion production, coherent scattering, etc., as G18 tunes, but implementation differs in many details (e.g., in RPA corrections, $W$ cutoff between the RES and DIS regions) and in values of the input parame-
Table 5 Standard and log-normal $\chi^{2} /$ ndf values calculated for the neutrino $\mathrm{CC} 0 \pi$ datasets on the flux-weighted differential cross sections on hydrocarbon target from T2K ND280 experiment [25] (see Figs. 19, 20). Calculations are done for several models using the full covariance matrix for the full $\mathrm{T} 2 \mathrm{~K}$ dataset (ndf $=93$ ) and for a subset of the data dependent only on the leptonic variables $($ ndf $=60$ ). Following to Ref. [25], only a part of the T2K data shown in Figs. 19 and 20 is included into the analysis. The number in parentheses for the T2K-tuned NEUT 5.4.0 model (incorporating "LFG+RPA" model with $1 p 1 h$ and $2 p 2 h$ prediction by Nieves et al. [28]) is taken from Ref. [25]

\begin{tabular}{|c|c|c|c|c|}
\hline \multirow[b]{2}{*}{ Model } & \multicolumn{2}{|c|}{$\begin{array}{l}\text { Full } \\
\text { Dataset }\end{array}$} & \multicolumn{2}{|c|}{$\begin{array}{l}\text { Leptonic } \\
\text { Variables } \\
\end{array}$} \\
\hline & $\frac{\chi_{\mathrm{st}}^{2}}{\mathrm{ndf}}$ & $\frac{\chi_{\log }^{2}}{\mathrm{ndf}}$ & $\frac{\chi_{\mathrm{st}}^{2}}{\mathrm{ndf}}$ & $\frac{\chi_{\log }^{2}}{\text { ndf }}$ \\
\hline $\mathrm{SMRFG}+M_{A}^{\text {run }}(\mathrm{hA} 2018)$ & 6.27 & 4.61 & 3.19 & 2.25 \\
\hline $\mathrm{SMRFG}+M_{A}^{\text {run }}(\mathrm{hN} 2018)$ & 5.77 & 4.45 & 3.39 & 2.93 \\
\hline G18_10a_02_11a & 4.19 & 4.46 & 2.17 & 2.87 \\
\hline G18_10b_02_11a & 4.22 & 4.51 & 2.18 & 2.90 \\
\hline T2K-tuned NEUT 5.4.0 & (3.99) & 4.63 & 2.90 & 3.77 \\
\hline GIBUU $2017(\mathscr{T}=0)$ & - & - & 2.83 & 2.45 \\
\hline GIBUU $2017(\mathscr{T}=1)$ & - & - & 3.90 & 2.75 \\
\hline
\end{tabular}

Table 6 Standard and log-normal $\chi^{2} /$ ndf values calculated for the distribution in the number of secondary protons with momenta above $500 \mathrm{MeV} / \mathrm{c}$ as measured by T2K ND280 [25] (see Fig. 20b). The NUWRO calculation is taken from Ref. [25]

\begin{tabular}{lll}
\hline Model & $\frac{\chi_{\mathrm{st}}^{2}}{\mathrm{ndf}}$ & $\frac{\chi_{\log }^{2}}{\mathrm{ndf}}$ \\
\hline SMRFG + $M_{A}^{\text {run }}(\mathrm{hA} 2018)$ & 2.76 & 2.22 \\
SMRFG + $M_{A}^{\text {run }}(\mathrm{hN} 2018)$ & 4.42 & 3.55 \\
G18_10a_02_11a & 7.29 & 8.30 \\
G18_10b_02_11a & 7.56 & 8.57 \\
NuWRO2019 & 2.07 & 2.77 \\
\hline
\end{tabular}

ters. The same is true for FSI; for example, NEUT 5.4.0 uses impulse approximation for the nucleon FSI and the model by Salcedo et al. [167] for pions which includes nuclear medium effects (cf. Sect. 4.1). For the leptonic data subset, the G18_10a_02_11a tune provides the lowest $\chi_{\mathrm{st}}^{2}$, while the SMRFG $+M_{A}^{\text {run }}$ model with hA 2018 FSI gives the lowest $\chi_{\log }^{2}$; the GIBUU2017 ( $\left.\mathscr{T}=0\right)$ predictions yield intermediate values of these criteria. It is worthy of note that the G18 tunes are less sensitive to the FSI model version than the $\mathrm{SMRFG}+M_{A}^{\text {run }}$ model. The greatest disagreement occurs for the full $\mathrm{T} 2 \mathrm{~K} \mathrm{C}_{8} \mathrm{H}_{8}$ dataset where the correlations drastically increase $\chi^{2} \mathrm{~s}$ for all models. A detailed comparison of several other models with the data has already been done in Ref. [25]. We only note that all these models also result in unacceptably large standard least-squares values for the full dataset: $\chi_{\mathrm{st}}^{2} / \mathrm{ndf}=4-6.2$. As is argued in Ref. [25], this analysis should be treated with caution. In particular, such $\chi_{\mathrm{st}}^{2}$ statistics can suffer from so-called Peelle's Pertinent Puz- 


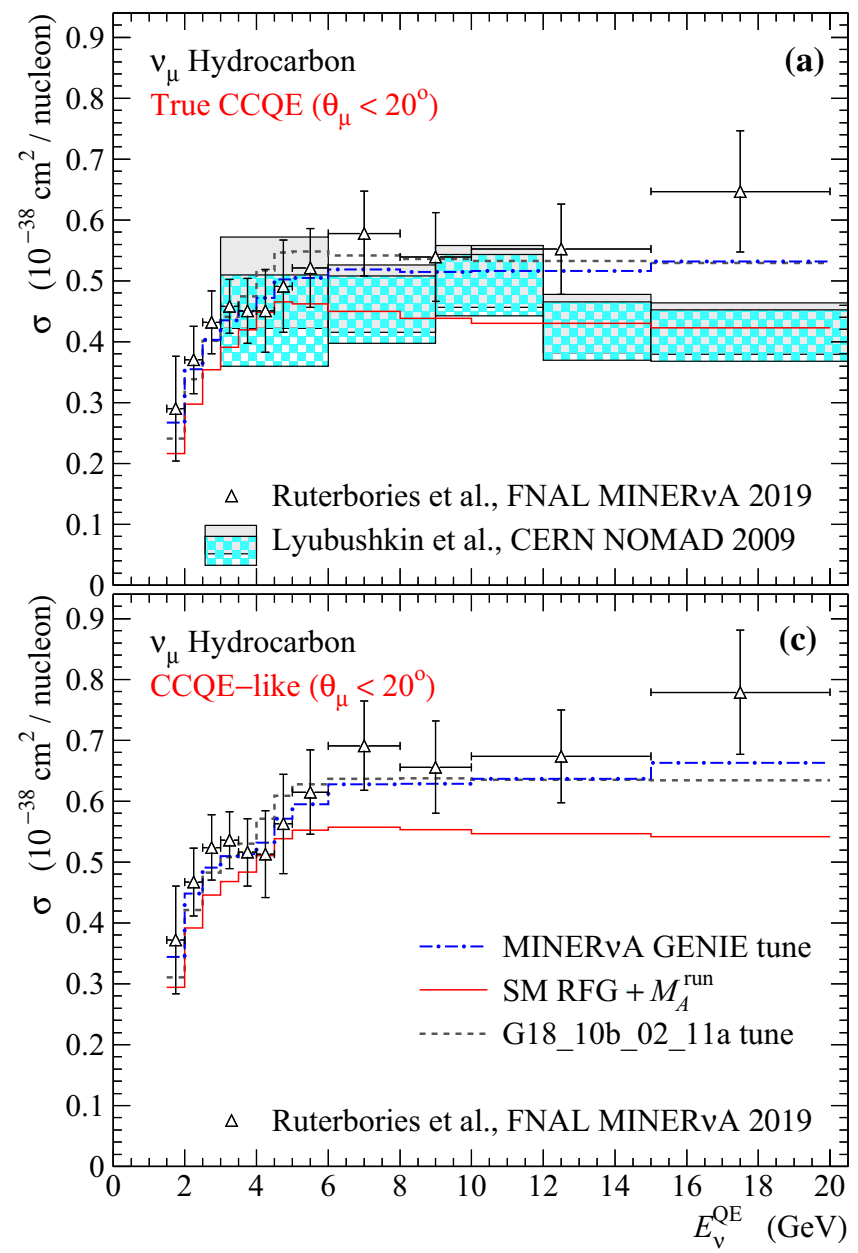

Fig. 21 Total cross sections vs. $E_{v}^{\mathrm{QE}}$ for the true CCQE (a, b) and CCQE-like (c, d) $v_{\mu}$ and $\bar{v}_{\mu}$ scattering from hydrocarbon as measured by MINER $v A$ [115,116]. Gray rectangles in the background represent the NOMAD data for carbon target [34] and translucent rectangles are the result of a conversion of the NOMAD data to the MINERvA target composition and kinematic cuts by applying the $\mathrm{SMRFG}+M_{A}^{\text {run }}$ model with hA2018 FSI. This conversions can only be thought of as

zle [214] (see Ref. [199] for a possible remedy to deal with this problem). Recall that the $\mathrm{SMRFG}+M_{A}^{\text {run }}$ models and G1 8 tunes satisfactory describe the T2K data on water target exposed to the same $v_{\mu}$ beam. Considering large statistical and systematic uncertainties, the relatively small expected differences between the $v_{\mu}$ scattering on oxygen and carbon are not very important. Thus, it can be assumed that the main distinctions between the two T2K measurements are in different event selection criteria and data-processing methods. Moreover, the predicted cross sections calculated as functions of muon variables agree with the corresponding data subset much better than ones for the data subset which includes the final-state proton variables. One may therefore expect that the enormous disagreement between the model predictions and the full $\mathrm{T} 2 \mathrm{~K} \mathrm{C}_{8} \mathrm{H}_{8}$ dataset is likely partially,

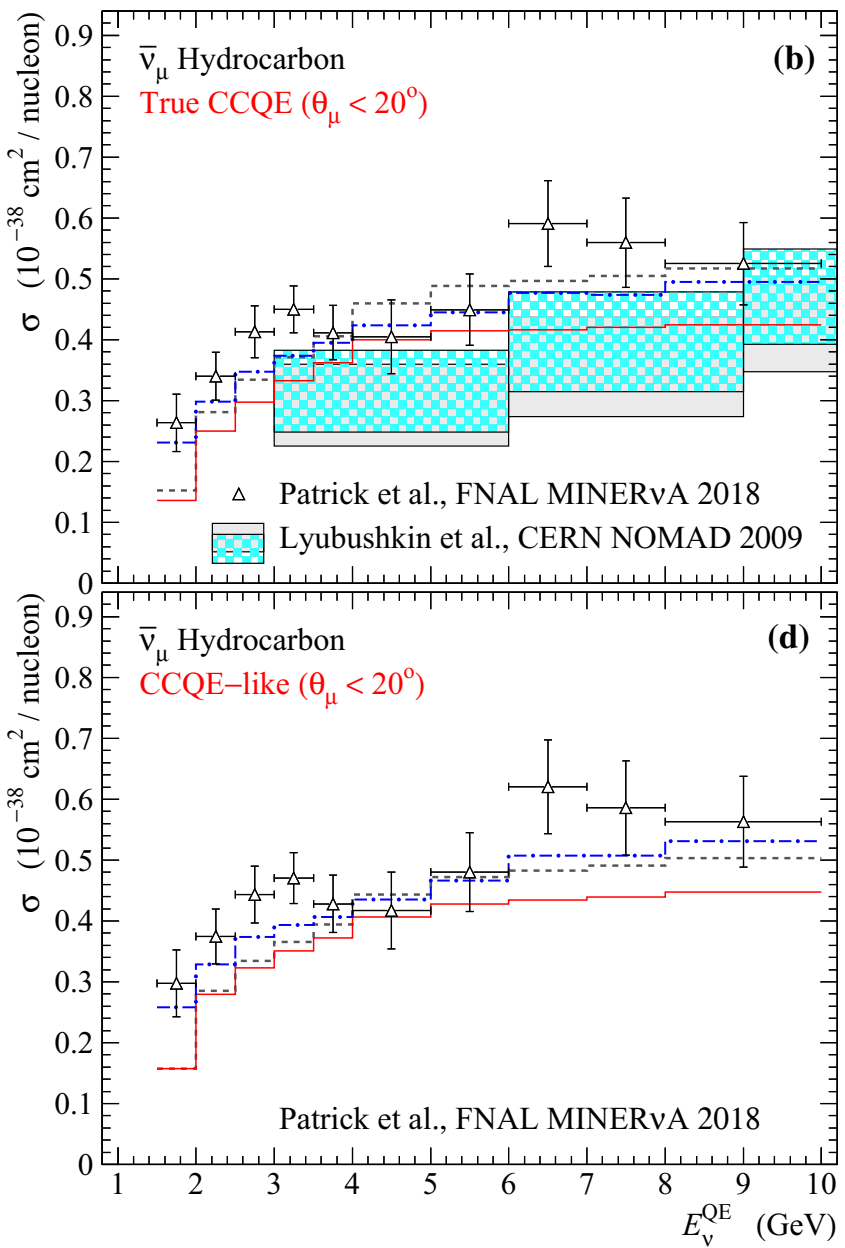

an approximation for a qualitative comparison of the NOMAD and MINER $v A$ results in the area of their intersection. The vertical error bars and heights of rectangles represent the total errors including the normalization uncertainties. Histograms represent the $\mathrm{SMRFG}+M_{A}^{\text {run }}$ model, G18_10b_02_11a tune, and the MINERvA-tuned GENIE v1 model from Refs. [115,116]. For more details, see Table 7

if not mainly, caused by the FSI problem common to all neutrino generators.

\subsection{MINERvA}

In this section, we discuss in detail the comparison between the experimental data of MINERvA [114-116] and predictions from a set of different theoretical models. The results of the statistical analysis are presented in Figs. 21, 22, 23, 24, 25 and 26 and Tables 7, 8, 9, 10 and 11. In all our calculations, we use the recent and most precise a priori prediction of the NuMI low-energy flux [215] based on a simulation that has been modified to reproduce thin and thick target measurements of meson and nucleon production as well as measurements of meson and nucleon absorption cross sec- 

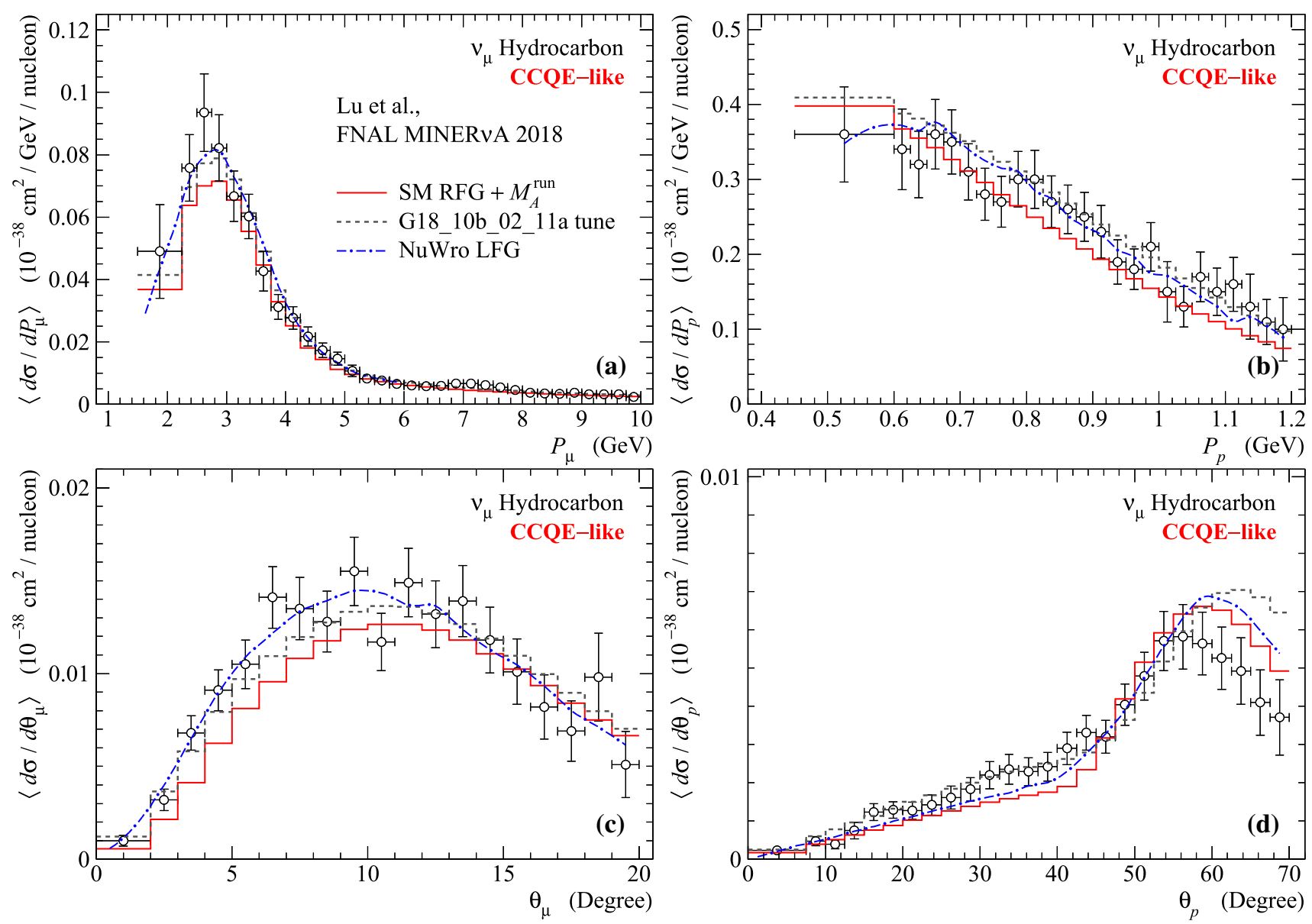

Fig. 22 Flux-weighted differential cross sections CCQE-like $v_{\mu}$ scattering from hydrocarbon as measured by MINER $v$ A [114] and plotted as functions of muon momentum, $P_{\mu}(\mathbf{a})$, proton momentum $P_{p}(\mathbf{b})$, muon scattering angle $\theta_{\mu}(\mathbf{c})$, and proton scattering angle $\theta_{p}(\mathbf{d})$. Vertical error bars represent the total errors including the normalization

tions. Although, for brevity, in the following we are talking about $v_{\mu}$ and $\bar{v}_{\mu}$ scattering from hydrocarbon target, in our simulations we actually accounted for the full chemical composition of the MINERvA detector, which is a mix of $88.51 \%$ carbon, $8.18 \%$ hydrogen, $2.5 \%$ oxygen, $0.47 \%$ titanium, $0.2 \%$ chlorine, $0.07 \%$ aluminum, and $0.07 \%$ silicon [115].

The signal in the recent MINER $v \mathrm{~A}$ measurements of the CCQE-like $v_{\mu} / \bar{v}_{\mu}$ interactions is usually defined [114-116] as an event which have post-FSI final states with one muon of angle $\theta_{\mu}<20^{\circ}$ with respect to the $v_{\mu} / \bar{v}_{\mu}$ beam when exiting the nucleus, no mesons and heavy or excited baryons, any number of photons with energy $\leq 10 \mathrm{MeV}$, and any number of protons or neutrons for incident neutrino, or with any number of protons having kinetic energy, $T_{p}$, below $120 \mathrm{MeV}$ for incident antineutrino. More specific kinematic constraints will be mentioned when necessary. uncertainty of $7.5 \%$. Histograms represent the $\mathrm{SMRFG}+M_{A}^{\text {run }}$ model and G18_10b_02_11a tune. Dot-dash curves are calculated with the NUWRO generator using the LFG model. Both the data and the NUWRO predictions are borrowed from ancillary files to Ref. [112]. For more details, see Table 8

Table 7 The values of the standard and log-normal $\chi^{2} /$ ndf calculated for the MINERvA neutrino [115] CCQE and CCQE-like datasets on the total cross sections $\sigma\left(E_{\nu}^{\mathrm{QE})}\right.$ ) (see Fig. 21). Calculations are done for several models using the full covariance matrices with $\mathrm{ndf}=12$

\begin{tabular}{|c|c|c|c|c|}
\hline \multirow[t]{2}{*}{ Model } & \multicolumn{2}{|c|}{ CCQE } & \multicolumn{2}{|c|}{ CCQE-like } \\
\hline & $\frac{\chi_{\mathrm{st}}^{2}}{\mathrm{ndf}}$ & $\frac{\chi_{\log }^{2}}{\mathrm{ndf}}$ & $\frac{\chi_{\mathrm{st}}^{2}}{\mathrm{ndf}}$ & $\frac{\chi_{\log }^{2}}{\mathrm{ndf}}$ \\
\hline $\mathrm{SMRFG}+M_{A}^{\text {run }}(\mathrm{hA} 2018)$ & 1.27 & 1.89 & 1.32 & 1.44 \\
\hline $\mathrm{SMRFG}+M_{A}^{\text {run }}(\mathrm{hN} 2018)$ & 1.28 & 1.91 & 1.41 & 1.63 \\
\hline G18_10a_02_11a & 1.35 & 1.46 & 1.40 & 1.22 \\
\hline G18_10b_02_11a & 1.39 & 1.50 & 1.41 & 1.31 \\
\hline MINER vA-tuned GENIE v1 & 0.77 & 0.89 & 0.73 & 0.65 \\
\hline
\end{tabular}




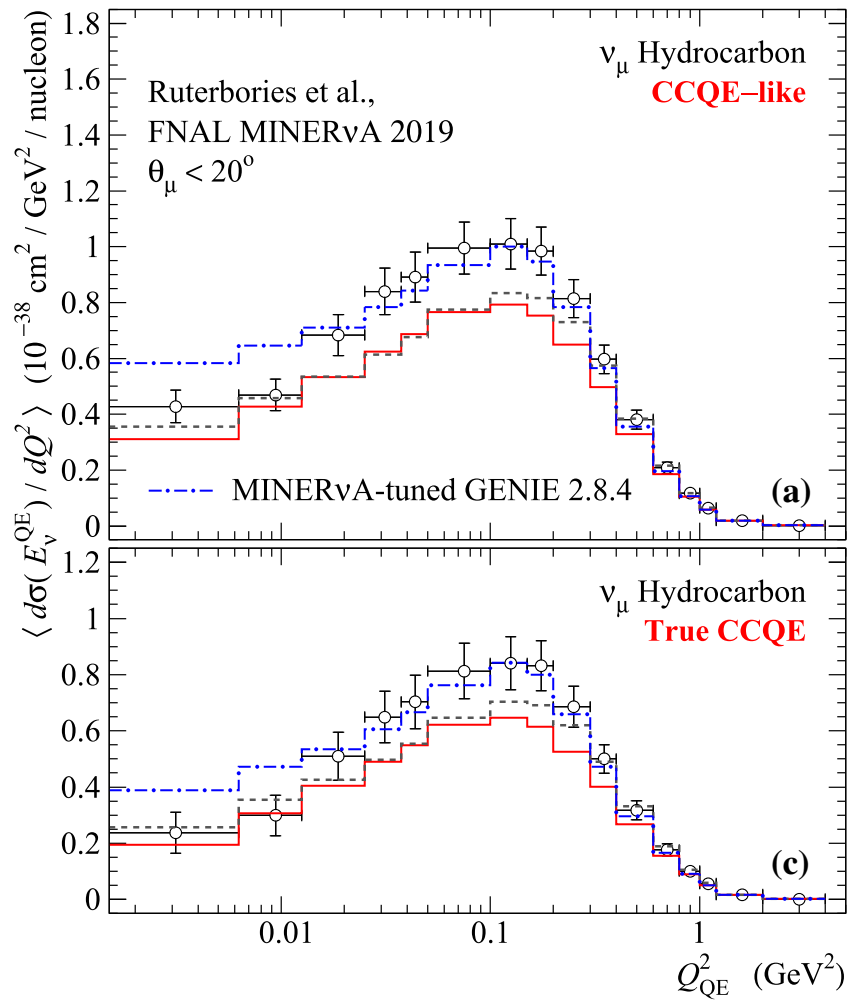

Fig. 23 Flux-weighted differential cross sections $d \sigma / d Q^{2}$ vs. $Q_{\mathrm{QE}}^{2}$ for the CCQE-like $(\mathbf{a}, \mathbf{b})$ and true CCQE $(\mathbf{c}, \mathbf{d}) v_{\mu}$ and $\bar{v}_{\mu}$ scattering from hydrocarbon as measured by MINERvA $[115,116]$. Vertical error bars represent the total errors including the normalization uncertainty

Table 8 The values of $\chi_{\mathrm{st}}^{2} /$ ndf and $\chi_{\log }^{2} /$ ndf (shown after slashes) calculated for the four MINER $v$ A CCQE-like datasets displayed in Fig. 22. Calculations are done with six models using the full covariance matrices. The columns "a", "b", "c", and "d" correspond to the panels with the same labels in Fig. 22; the last column shows the $\chi_{\text {st }}^{2} /$ ndf and $\chi_{\log }^{2} /$ ndf evaluated for the full data set involving all four data subsets (assuming

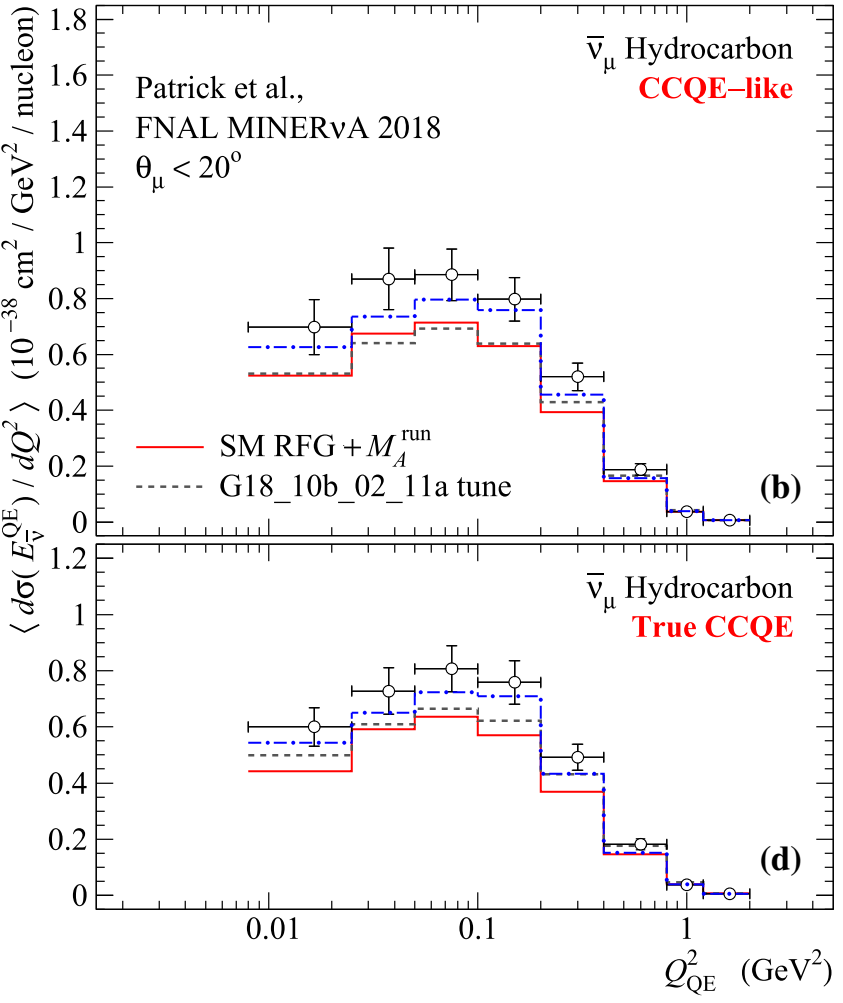

of $7.5 \%$. Histograms and curves represent the $\mathrm{SMRFG}+M_{A}^{\text {run }}$ model and G18_10b_02_11a tune predictions. Also shown the MINERvAtuned GENIE v1 model from Refs. [115,116]. For more details, see Table 9

no correlations between them). The ndf values are given in parentheses (second row). Asterisks mark the NUWRO calculations made using local Fermi gas (LFG) and spectral function (SF) models, and covering incomplete data subsets (ndf $=16$ and 86 for, respectively, the second and last columns). The NuWro predictions are taken from the ancillary files of Ref. [112]

\begin{tabular}{llllll}
\hline Model & $\begin{array}{l}\mathrm{a} \\
(32)\end{array}$ & $\begin{array}{l}\mathrm{b} \\
(19)\end{array}$ & $\begin{array}{l}\mathrm{c} \\
(25)\end{array}$ & $\begin{array}{l}\mathrm{d} \\
(26)\end{array}$ \\
\hline SMRFG+ $M_{A}^{\text {run }}(\mathrm{hA} 2018)$ & $0.89 / 1.08$ & $2.05 / 2.52$ & $1.23 / 1.33$ & $2.36 / 2.48$ \\
SMRFG+M $M_{A}^{\text {run }}(\mathrm{hN} 2018)$ & $0.92 / 1.12$ & $1.84 / 2.19$ & $1.47 / 1.54$ & $2.41 / 2.62$ & $1.56 / 1.77$ \\
G18_10a_02_11a & $1.07 / 1.02$ & $1.93 / 1.77$ & $1.27 / 0.99$ & $3.01 / 1.68$ & $1.61 / 1.80$ \\
G18_10b_02_11a & $1.06 / 1.01$ & $1.85 / 1.64$ & $1.50 / 1.13$ & $2.99 / 1.70$ & $1.77 / 1.32$ \\
NuWRO (LFG) & $1.29 / 1.19^{*}$ & $1.21 / 1.15$ & $0.99 / 1.01$ & $1.99 / 1.81$ & $1.81 / 1.33$ \\
NUWRO (SF) & $1.03 / 1.21^{*}$ & $1.13 / 1.25$ & $0.84 / 1.00$ & $1.29 / 1.21$ & $1.08 / 1.16^{*}$ \\
\hline
\end{tabular}

\subsubsection{Total CCQE and CCQE-like cross sections}

In Fig. 21, we show the comparison of the MINER $v \mathrm{~A}$ data $[115,116]$ with three model predictions for the total CCQE (panels $(\mathrm{a}, \mathrm{b})$ ) and CCQE-like (panels $(\mathrm{c}, \mathrm{d})$ ) total neutrino and antineutrino interaction cross sections on hydrocarbon as functions of $E_{\nu}^{\mathrm{QE}}$. Along with the $\mathrm{SMRFG}+M_{A}^{\text {run }}$ model with hA2018 FSI and G18_10b_02_11a tune, we also examine a MINERvA-tuned GENIE 2.8.4 model (see Fig. 21), which incorporates RPA and tuned $2 p 2 h$ and is used as default in the MINER $v \mathrm{~A}$ analysis for extracting the cross sections. The MINER $v \mathrm{~A}$ measurements fill the gap between the modern low-energy (MiniBooNE, SciBooNE, T2K) and high-energy (NOMAD, LAr-TPC) data and it is instructive to look at their intersection. For this purpose, panels $(a, b)$ also show the NOMAD data [34]. 

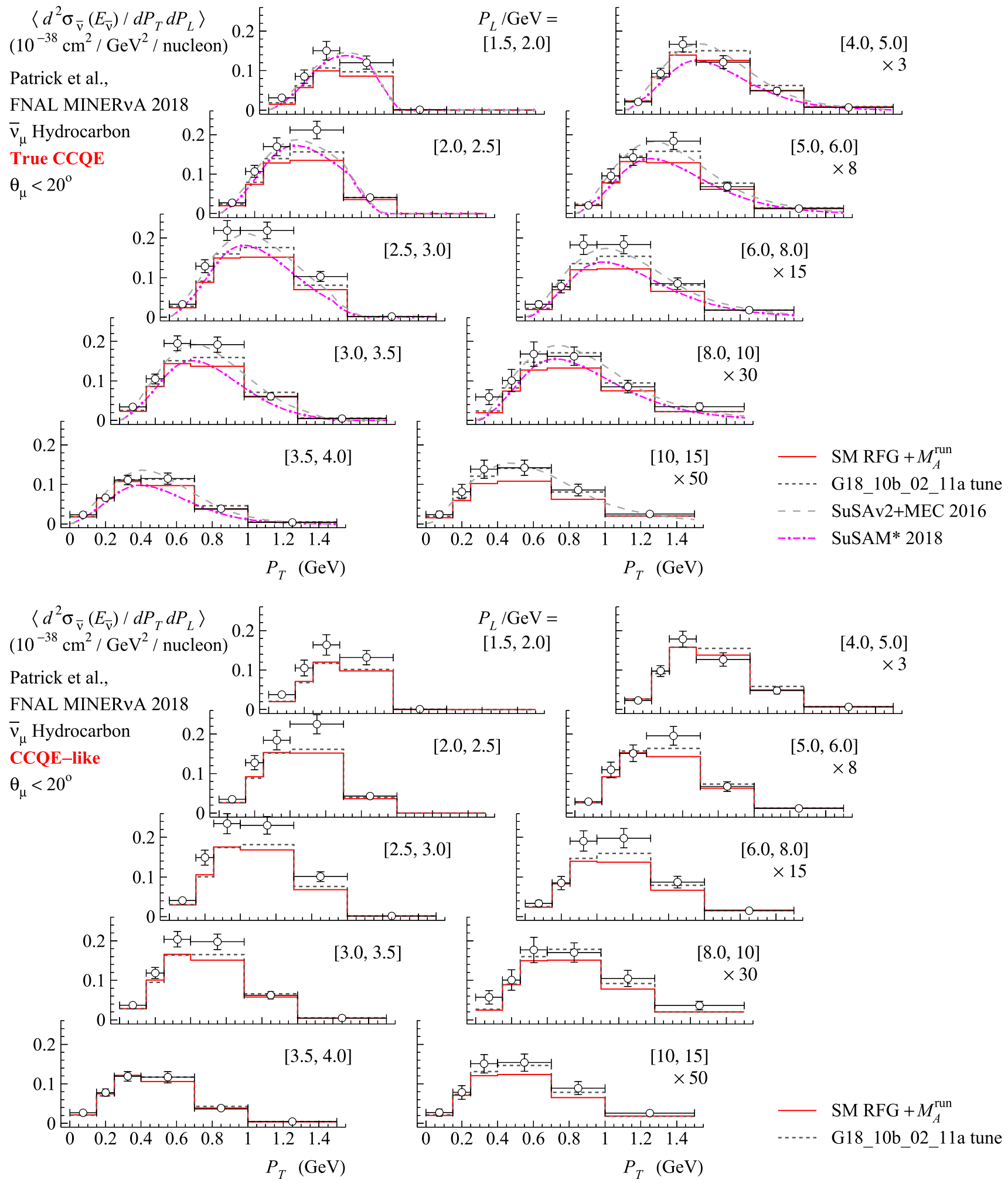

Fig. 24 Flux-weighted double-differential cross sections, $d^{2} \sigma / d P_{T} d P_{L}$, for the CCQE (ten top panels) and CCQE-like (ten bottom panels) $\bar{v}_{\mu}$ scattering from hydrocarbon as measured by MINERvA [116] and plotted vs. transverse muon momentum, $P_{T}$, for several intervals of the longitudinal momentum, $P_{L}$ (shown in square brackets). Vertical error bars represent the total errors including the normalization uncertainty of $7.5 \%$. Histograms represent the SMRFG $+M_{A}^{\text {run }}$ model and G18_10b_02_11a tune, curves show SuSAv2-MEC 2016 [65] and SuSAM* 2018 [68] models. See Table 10 


$$
\begin{array}{cc}
\left\langle d^{2} \sigma_{v}\left(E_{v}\right) / d P_{T} d P_{L}\right\rangle & 0.4 \\
\left(10^{-38} \mathrm{~cm}^{2} / \mathrm{GeV}^{2} / \text { nucleon }\right) & 0.2
\end{array}
$$

Ruterbories et al.,

FNAL MINERvA 2019

$v_{\mu}$ Hydrocarbon

True CCQE

$\theta_{\mu}<20^{\circ}$
$0.4=$
$P_{T} / \mathrm{GeV}=$

$[0.000,0.075]$
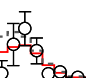

q
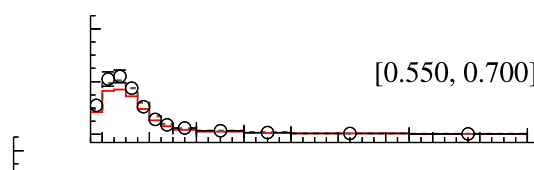

$[0.075,0.150]$

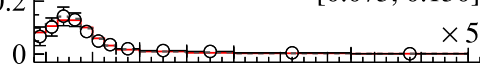

$\times 5$

$[0.150,0.250]$
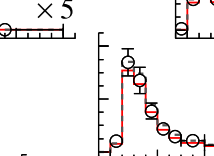

$[0.700,0$ 850]
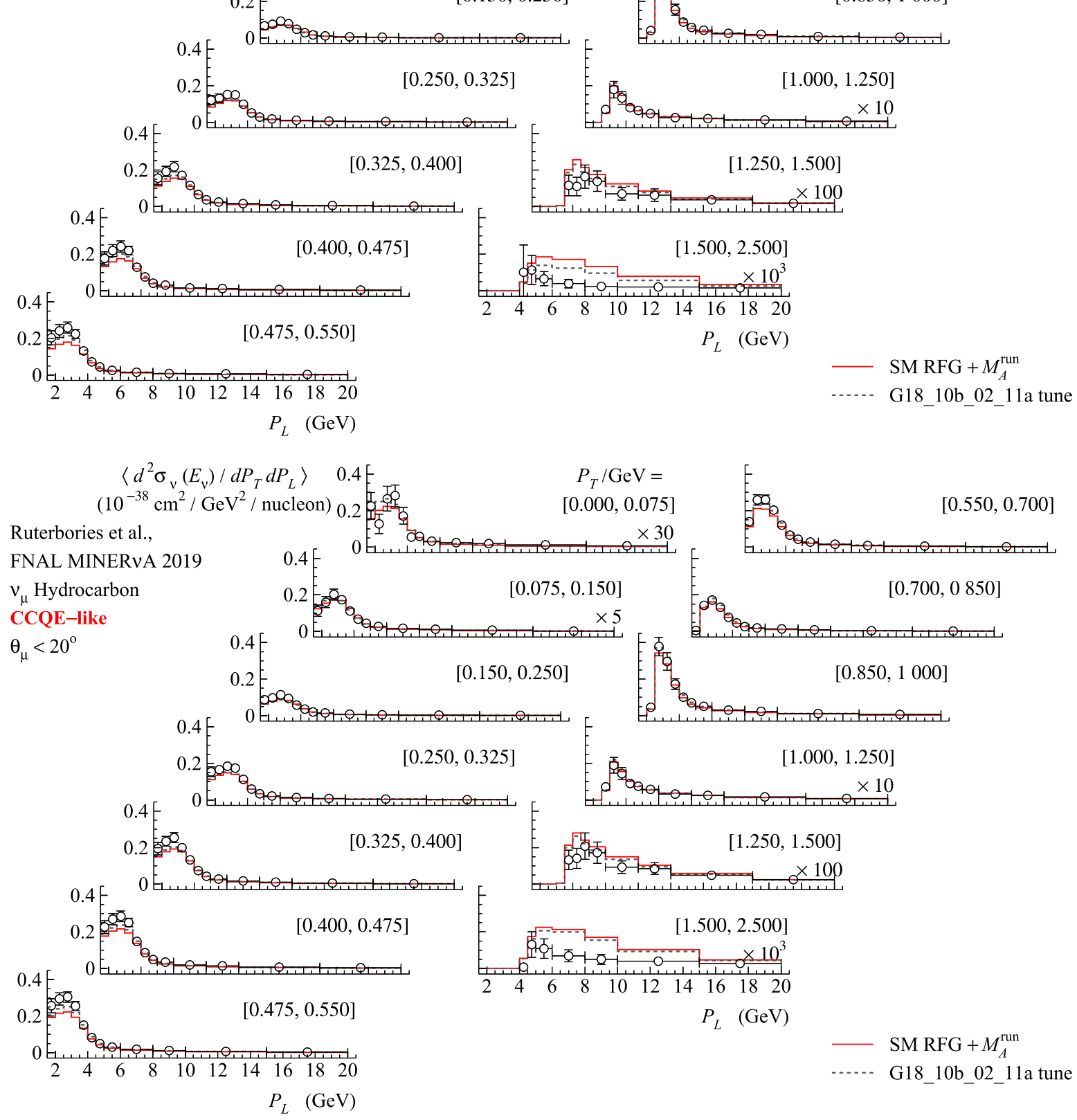

Fig. 25 Flux-weighted double-differential cross sections, $d^{2} \sigma / d P_{T} d P_{L}$, for the CCQE (thirteen top panels) and CCQE-like (thirteen bottom panels) $v_{\mu}$ scattering from hydrocarbon as measured by MINER $v \mathrm{~A}$ [115] and plotted vs. transverse muon momentum, $P_{T}$, for several intervals of the longitudinal momentum, $P_{L}$ (shown in square brackets). Vertical error bars represent the total errors including the normalization uncertainty of $7.5 \%$. Histograms represent the SMRFG $+M_{A}^{\text {run }}$ model and G18_10b_02_11a tune. For more details, see Table 10 


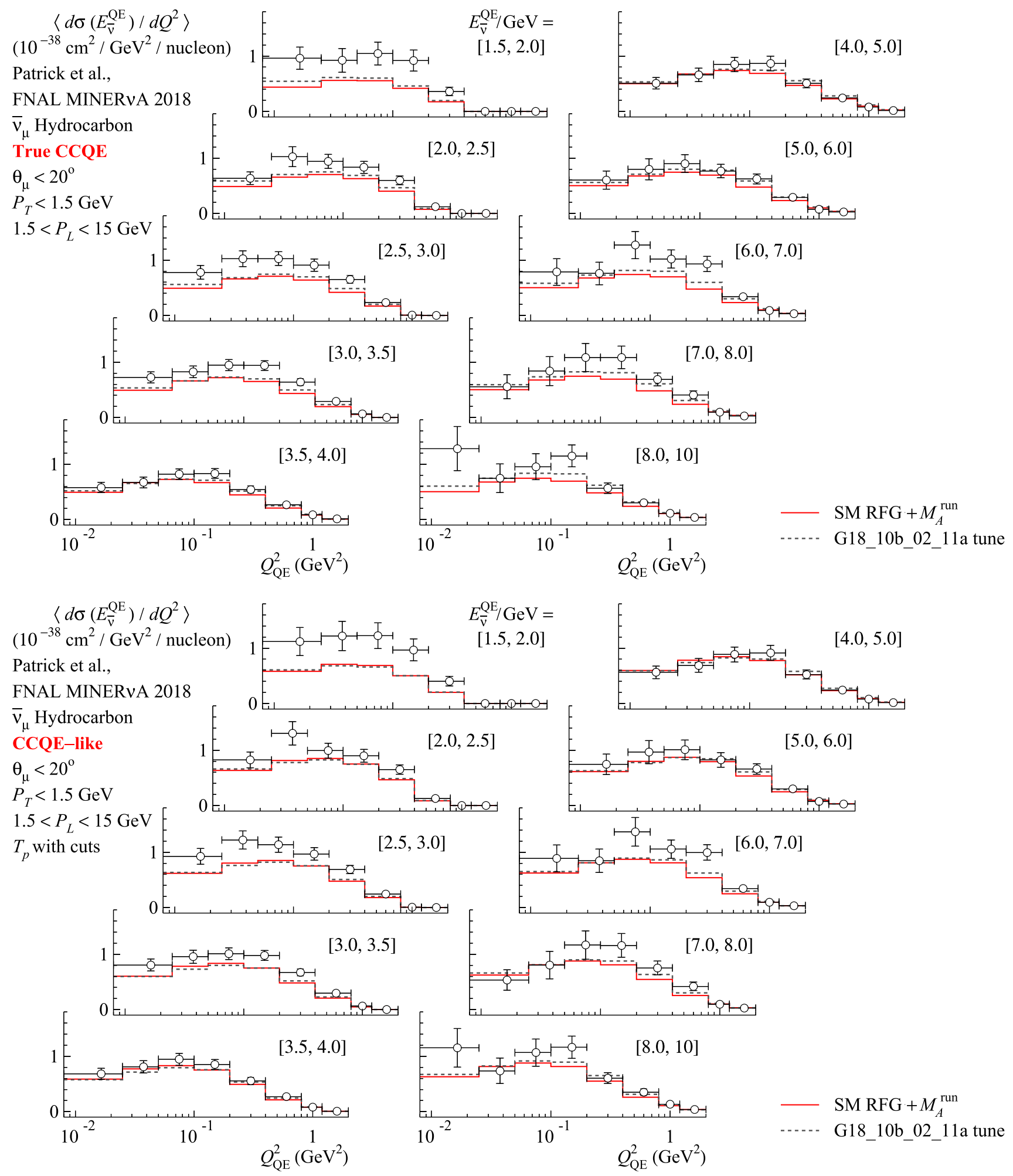

Fig. 26 Flux-weighted double-differential cross sections for the CCQE (ten top panels) and CCQE-like (ten bottom panels) $\bar{v}_{\mu}$ scattering from hydrocarbon as measured by MINER $v \mathrm{~A}[116]$ and plotted as functions of $Q_{\mathrm{QE}}^{2}$ for several intervals of the antineutrino energy
$E_{\bar{v}_{\mu}}^{\mathrm{QE}}$ (shown in square brackets) The vertical error bars represent the total errors including the normalization uncertainty of $7.5 \%$. Histograms represent the SMRFG $+M_{A}^{\text {run }}$ model and G18_10b_02_11a tune. For more details, see Table 11 
Table 9 The values of the standard and log-normal $\chi^{2} /$ ndf calculated for the MINER $v$ A neutrino [115] CCQE and CCQE-like datasets on the flux-weighted differential cross sections $d \sigma / d Q^{2}$ (see Fig. 23). Calculations are done for several models using the full covariance matrices with ndf $=16$

\begin{tabular}{|c|c|c|c|c|}
\hline \multirow{2}{*}{ Model } & \multicolumn{2}{|c|}{ CCQE } & \multicolumn{2}{|c|}{ CCQE-like } \\
\hline & $\frac{\chi_{\mathrm{st}}^{2}}{\mathrm{ndf}}$ & $\frac{\chi_{\log }^{2}}{\text { ndf }}$ & $\frac{\chi_{\mathrm{st}}^{2}}{\mathrm{ndf}}$ & $\frac{\chi_{\log }^{2}}{\text { ndf }}$ \\
\hline $\mathrm{SMRFG}+M_{A}^{\text {run }}(\mathrm{hA} 2018)$ & 1.75 & 1.95 & 1.31 & 1.46 \\
\hline $\mathrm{SMRFG}+M_{A}^{\text {run }}(\mathrm{hN} 2018)$ & 1.73 & 1.92 & 1.11 & 1.47 \\
\hline G18_10a_02_11a & 2.43 & 2.77 & 2.12 & 2.72 \\
\hline G18_10b_02_11a & 2.43 & 2.77 & 2.21 & 2.99 \\
\hline MINER $v$ A-tuned GENIE v1 & 2.90 & 2.08 & 4.02 & 3.08 \\
\hline
\end{tabular}

A comparison of the MINER $v$ A data with other recent measurements is shown in Fig. 28 (see Appendix B). As is explained in Ref. [116], the MINER $v$ A measurements under consideration do not in fact yield exactly the total CCQE-like cross sections, $\sigma_{\text {tot }}$, but rather a single-differential projection of the double-differential cross sections, i.e. a well-defined approximation to $\sigma_{\text {tot }}$. This is in part because the RFG-based quantity $E_{v}^{\mathrm{QE}}$ does not of course match the true (anti)neutrino energy $E_{\nu}^{\text {True }}$ (and it is not applicable to the contribution from the CCQE $\bar{v}_{\mu}$ scattering off hydrogen). However, as mentioned earlier, the quantities $E_{v}^{\mathrm{QE}}$ and $E_{v}^{\text {True }}$ are closely correlated. Considering all this, we have performed accurate simulations with the kinematic restrictions reproducing the MINER $v$ A definition of $\sigma\left(E_{v}^{\mathrm{QE}}\right)$ as truly as possible.

A direct quantitative comparison of the MINER $v \mathrm{~A}$ and NOMAD data is complicated by the kinematic cuts used in the MINER $v \mathrm{~A}$ analysis and by the difference in the chemical compositions of the two detector targets. The cuts considerably reduces the total CCQE cross section at low energies while becomes almost inessential above 5-6 GeV; the difference in the composition has a small effect at all energies. To qualitatively compare the MINER $v$ A and NOMAD data, we converted the latter by multiplying bin-by-bin the original NOMAD data on a carbon-reach target (shown by gray rectangles in panels (a, b) of Fig. 21) by the factor

$\sigma^{\operatorname{MINERvA}}\left(E_{v}^{\mathrm{QE}}\right) / \sigma^{\operatorname{NOMAD}}\left(E_{v}^{\mathrm{QE}}\right)$,

where $\sigma^{\text {MINERvA }}$ is the total CCQE cross section calculated for the MINER $v$ A detector target using the aforementioned cuts (the main of which is the cut on the muon scattering angle) and $\sigma^{\text {NOMAD }}$ is the same cross section on carbon calculated for the full phase space. Both calculations are done using the SMRFG $+M_{A}^{\text {run }}$ model with hA 2018 FSI and the result is shown by translucent rectangles. Note that the conversion factor (15) decreases the NOMAD neutrino cross section and increases the antineutrino one, although the effects due to the differences in the detector targets and
Table 10 The values of the standard and log-normal $\chi^{2} /$ ndf calculated for the MINERvA neutrino [115] and antineutrino [116] CCQE and CCQE-like datasets on the flux-weighted double-differential cross sections $d^{2} \sigma / d P_{T} d P_{L}$ (see Fig. 24). Calculations are done for several models using the full covariance matrices

\begin{tabular}{|c|c|c|c|c|}
\hline \multirow[t]{2}{*}{ Model } & \multicolumn{2}{|c|}{ CCQE } & \multicolumn{2}{|c|}{ CCQE-like } \\
\hline & $\frac{\chi_{\mathrm{st}}^{2}}{\mathrm{ndf}}$ & $\frac{\chi_{\log }^{2}}{\operatorname{ndf}}$ & $\frac{\chi_{\mathrm{st}}^{2}}{\mathrm{ndf}}$ & $\frac{\chi_{\log }^{2}}{\mathrm{ndf}}$ \\
\hline \multicolumn{5}{|l|}{ Neutrino dataset $(\mathrm{ndf}=144)$} \\
\hline $\mathrm{SMRFG}+M_{A}^{\text {run }}(\mathrm{hA} 2018)$ & 1.66 & 1.53 & 1.73 & 1.81 \\
\hline $\mathrm{SMRFG}+M_{A}^{\mathrm{run}}(\mathrm{hN} 2018)$ & 1.65 & 1.52 & 1.49 & 1.86 \\
\hline G18_10a_02_11a & 1.43 & 1.20 & 1.48 & 1.32 \\
\hline G18_10b_02_11a & 1.45 & 1.22 & 1.26 & 1.34 \\
\hline MINER $v$ A-tuned GENIE & 1.51 & 1.04 & 1.77 & 1.41 \\
\hline \multicolumn{5}{|l|}{ Antineutrino dataset $(\mathrm{ndf}=58)$} \\
\hline $\mathrm{SMRFG}+M_{A}^{\mathrm{run}}(\mathrm{hA} 2018)$ & 1.34 & 2.22 & 1.49 & 2.06 \\
\hline $\mathrm{SMRFG}+M_{A}^{\text {run }}(\mathrm{hN} 2018)$ & 1.34 & 2.11 & 1.49 & 2.14 \\
\hline G18_10a_02_11a & 1.46 & 1.89 & 1.44 & 1.74 \\
\hline G18_10b_02_11a & 1.44 & 1.85 & 1.40 & 1.72 \\
\hline SuSAv2-MEC 2018 & 2.13 & 2.02 & - & - \\
\hline SuSAM* 2018 & 1.51 & 2.64 & - & - \\
\hline MINER $v$ A-tuned GENIE & 1.58 & 1.85 & 1.40 & 1.70 \\
\hline
\end{tabular}

kinematic cuts partially compensate each other. Despite the roughness and model dependence of such a conversion, a tension between the MINER $v \mathrm{~A}$ and NOMAD data is plainly visible both in normalization and in shape. The FSI model applied to the data calculation has little or no effect on this tension. Similar qualitative comparison of the MINER $v$ A and properly rescaled MiniBooNE total CCQE cross sections indicates no significant tension between these data within the errors. Figure 21 demonstrates (with minor reservations) reasonable agreement between the MINER $v$ A data and both G18_10b_02_11a tune and MINERvA-tuned GENIE v1 model [115,116], but also a clear conflict of the data with the $\mathrm{SMRFG}+M_{A}^{\text {run }}$ model, which significantly underestimates the measured cross sections in both neutrino and antineutrino cases. Renormalization of the data does not essentially improve the situation due to the inconsistency presented also in the shapes. The fact that $\mathrm{SMRFG}+M_{A}^{\text {run }}$ model describes the NOMAD (participated in the adjusting of the $M_{A}^{\text {run }}$ parameters) and fails in description of the MINER $v A$ data in the region of their intersection indicates a contradiction between these datasets. To quantify these findings, it is necessary to take into account strong correlations between the MINER $v$ A data points. This is not possible for the antineutrino case due to the unavailability of the covariance matrix for this dataset. For the neutrino case, the comparison is shown in Table 7 which collects the standard and log-normal $\chi^{2} /$ ndf values calculated with the full covariance matrix. It is seen that the tension is partially softened after 
accounting for the correlations. All four GENIE 3 models listed in the Table have comparable $\chi^{2}$ s indicating a small tension rather than contradiction with the MINER $\nu \mathrm{A}$ results. Consistency between the standard and log-normal $\chi^{2} \mathrm{~s}$ indicates the reliability of both statistical tests. The variations due to the FSI models are comparatively small and not statistically significant to make a choice between these models. The MINERvA-tuned GENIE v1 model $[115,116]$ achieves ("by definition") the best agreement with the data. This model (adjusted to the MINER $v \mathrm{~A}$ data) is a modified version of the GENIE 2.8.4 default model, which in particular, reduces the standard, $1 p 1 h$, CCQE cross section (by applying the RPA corrections), enhances the $2 p 2 h$ contribution (by about $53 \%$, when integrated over the whole phase space), and decreases the non-resonant pion production (by about $43 \%$ ). Just like the GENIE 3 tunes G18_10a_02_11a and G18_10b_02_11a, both $2 p 2 h$ and RPA contributions in the MINER $v$ A-tuned $v 1$ model are the parts of the Valencia model [185-187]. Thus, we can conclude that the distinctions between the models are due mainly to different re-weighting of the $2 p 2 h$ and RPA parts.

\subsubsection{Single-differential cross sections}

A comparison of the flux-weighted differential CCQE-like $\nu_{\mu}$ cross sections $d \sigma / d P_{\mu}, d \sigma / d P_{p}, d \sigma / d \theta_{\mu}$, and $d \sigma / d \theta_{p}$ measured by MINER $v$ A [114] with the respective predictions of three models, SM RFG $+M_{A}^{\text {run }}$, G18_10b_02_11a, and NUWRo LFG, is shown in Fig. 22. Here $P_{\mu}$ and $\theta_{\mu}\left(P_{p}\right.$ and $\theta_{p}$ ) are, respectively, the muon (proton) momentum and scattering angle.

The additional requirements for the MINER $\nu$ A signal definition include $1.50 \mathrm{GeV}<P_{\mu}<10 \mathrm{GeV}, \theta_{\mu}<20^{\circ}$, $0.45 \mathrm{GeV}<P_{p}<1.2 \mathrm{GeV}, \theta_{p}<70^{\circ}$; there must be at least one final-state proton satisfying the above conditions. At first glance, the solid histogram corresponding to the $\mathrm{SMRFG}+M_{A}^{\text {run }}$ model shown in panel (a) describes the experimental data $\left(d \sigma / d P_{\mu}\right)$ at small muon momenta worse than two other models. For other panels, it is more difficult to tell by eye which model best describes the experimental data. Therefore, it is better to refer to Table 8 , in which we present the standard and log-normal $\chi^{2} /$ ndf values for these and three other models. All calculations take into account the full correlation matrices. As one can see from the table, the NUWRO SF model has the least $\chi_{\text {st }}^{2} /$ ndf for all data subsets except those shown in panel (a). The NUWRO LFG model demonstrates a little worse results, but in general better than the other models everywhere, except the case shown in panel (a), where just the $\mathrm{SMRFG}+M_{A}^{\text {run }}$ model shows the best $\chi_{\text {st }}^{2} /$ ndf value. This example clearly demonstrates the discrepancy between the visual assessment of the quality of the data description and a calculation that takes into account the play of correlations. Comparing the values of $\chi_{\text {st }}^{2} /$ ndf for the full dataset presented in Fig. 22 (last column in Table 8) one finds that the NUWRO results obtained with the LFG and SF models show the least $\chi_{\text {st }}^{2} /$ ndf values, the G18 tunes give the worst ones, and the $\chi_{\mathrm{st}}^{2} /$ ndf values for the $\mathrm{SMRFG}+M_{A}^{\text {run }}$ model lie between them. What concerns the $\chi_{\log }^{2} /$ ndf values for the full dataset, here again the two NUWRO versions show the best result, but the worst result in this case is shown by the SMRFG $+M_{A}^{\text {run }}$ models. In all cases, the description is slowly sensitive to the FSI model.

The flux-weighted cross sections $d \sigma / d Q_{\mathrm{QE}}$ for the $v_{\mu}$ and $\bar{v}_{\mu}$ scattering from hydrocarbon along with few model predictions are shown in Fig. 23, where the two top (bottom) panels display the CCQE-like (CCQE) datasets. The $\chi_{\text {st }}^{2} /$ ndf and $\chi_{\log }^{2} /$ ndf values for several models are listed in Table 9 (for the neutrino case only). Although by eye the $\mathrm{SMRFG}+M_{A}^{\text {run }}$ model describes the data worse than the G18 tune and MINERvA-tuned GENIE, the quantitative comparison in Table 9 shows a completely opposite picture, also illustrating the unpredictable effect of correlations.

A comparison of the flux-weighted differential cross sections $d \sigma / d p_{T}$ and $d \sigma / d p_{L}$ (where $p_{T}$ and $p_{L}$ are the muon transverse and longitudinal momenta) for the true CCQE and CCQE-like $v_{\mu}$ and $\bar{v}_{\mu}$ scattering from hydrocarbon $[115,116]$ shows that the G18 tune and MINER $v A$-tuned GENIE both give a reasonable description of the data while the SMRFG $+M_{A}^{\text {run }}$ model underestimates the MINER $v \mathrm{~A}$ cross sections. We do not discuss here the corresponding $\chi^{2} \mathrm{~s}$ since these datasets represent just the one-dimensional slices from the double-differential cross sections shown below in Figs. 24, 25 and 26 and the covariance matrices for these slices are not provided by the authors.

\subsubsection{Double-differential cross sections}

The MINER $v$ A results for antineutrino scattering on hydrocarbon [116] with several predictions are shown in Fig. 24. The CCQE and CCQE-like flux-weighted double-differential cross sections $d^{2} \sigma / d P_{T} d P_{L}$ are presented at ten top and ten bottom panels, respectively, for the fixed intervals of the muon longitudinal momentum $P_{L}$ (shown in square brackets) as functions of the muon transverse momentum $P_{T}$; the data are compared with the four models as listed in the legend. Similar plots for the neutrino CCQE and CCQE-like datasets [115] are presented in Fig. 25 as functions of $P_{L}$ for the fixed intervals of $P_{T}$; the data are compared with the two models. The signal definition for CCQE-like events in this case is post-FSI CC-events with $\theta_{\mu}<20^{\circ}$, any numbers of nucleons and low-energy photons (with energies below $10 \mathrm{MeV}$ ) and, as above, without mesons and heavy or excited baryons. In the case of the $v_{\mu} / \bar{v}_{\mu}$ true CCQE signal, only the cuts on leptonic variables are essential.

The values of the reduced standard and log-normal $\chi^{2} \mathrm{~s}$ the models presented in are summarized in Table 10. The 
Table 11 The values of the standard and log-normal $\chi^{2} /$ ndf calculated for the MINER $v \mathrm{~A}$ antineutrino CCQE and CCQE-like datasets on the flux-weighted double-differential cross sections $d^{2} \sigma / d Q_{\mathrm{QE}}^{2} d E_{\nu}^{\mathrm{QE}}$ (see Fig. 26 and $d^{2} \sigma / d Q_{\mathrm{QE}}^{2} d E_{v}^{\text {True }}$ (not illustrated) [116]. Calculations are done for several models using a simplified (block-diagonal) covariance matrices with 74 and 76 degrees of freedom for the cross sections represented in terms of $E_{v}^{\mathrm{QE}}$ and $E_{v}^{\text {True }}$, respectively. The reasons for the modification of the covariance matrices are explained in the main text

Model

\begin{tabular}{|c|c|c|c|}
\hline \multicolumn{2}{|c|}{$E_{v}^{\mathrm{QE}}$} & \multicolumn{2}{|c|}{$E_{v}^{\text {True }}$} \\
\hline$\chi_{\mathrm{st}}^{2}$ & $\chi_{\log }^{2}$ & $\chi_{\mathrm{st}}^{2}$ & $\chi_{\log }^{2}$ \\
\hline$\overline{\mathrm{ndf}}$ & $\overline{\mathrm{ndf}}$ & $\overline{\mathrm{ndf}}$ & $\overline{\mathrm{ndf}}$ \\
\hline
\end{tabular}

CCQE dataset

\begin{tabular}{lllll} 
SMRFG $+M_{A}^{\text {run }}(\mathrm{hA} 2018)$ & 0.58 & 0.53 & 0.58 & 0.54 \\
SMRFG $+M_{A}^{\text {run }}(\mathrm{hN} 2018)$ & 0.60 & 0.55 & 0.60 & 0.55 \\
G18_10a_02_11a & 0.56 & 0.37 & 0.27 & 0.35 \\
G18_10b_02_11a & 0.56 & 0.37 & 0.29 & 0.49 \\
SuSAM*2018 & 0.05 & 0.21 & - & - \\
CCQE-like dataset & & & & \\
SMRFG+ $M_{A}^{\text {run }}(\mathrm{hA} 2018)$ & 0.54 & 0.86 & 0.58 & 0.86 \\
SMRFG+ $M_{A}^{\text {run }}(\mathrm{hN} 2018)$ & 0.62 & 0.96 & 0.66 & 0.94 \\
G18_10a_02_11a & 0.61 & 0.86 & 0.48 & 0.97 \\
G18_10b_02_11a & 0.67 & 0.93 & 0.54 & 1.14 \\
\hline
\end{tabular}

$\mathrm{SMRFG}+M_{A}^{\text {run }}$ model and G18 tunes are presented for the two FSI versions. The table also includes the corresponding numbers for the MINERvA-tuned GENIE2.8.4 model, which was used in the MINERvA experiment for extracting the cross sections. The calculations were carried out using the full covariance matrices. Note that our estimation of $\chi_{\mathrm{st}}^{2} / \mathrm{ndf}$ for the SuSAv2-MEC model ( $\bar{v}_{\mu}$ case, CCQE) differs noticeably from author's result of 1.79 [66]. Overall, the two G18 tunes provide the best description of the CCQE and CCQElike data for both $\bar{v}_{\mu}$ and $v_{\mu}$ cases; this can be seen both from the figures and from the comparison of the standard and log-normal $\chi \mathrm{s}$ in Table 10. As for other models, then the $\mathrm{SMRFG}+M_{A}^{\text {run }}$ shows better agreement in terms of $\chi_{\mathrm{st}}^{2} / \mathrm{ndf}$ for the $\bar{v}_{\mu}$ CCQE case, and MINER $v$ A-tuned GENIE $v 1$ has the lowest values of $\chi_{\mathrm{st}}^{2} / \mathrm{ndf}$ for $v_{\mu} \mathrm{CCQE}$ and of $\chi_{\log }^{2} / \mathrm{ndf}$ for the $\bar{v}_{\mu}$ CCQE-like case.

The CCQE and CCQE-like flux-weighted $\bar{v}_{\mu}$ doubledifferential cross sections of the same data as shown in Fig. 24 with the same cuts [116] but for other kinematic variables are plotted as functions of $Q_{\mathrm{QE}}^{2}$ for several intervals of the fixed energy $E_{\bar{v}_{\mu}}^{\mathrm{QE}}$ (Fig. 26). (the energy bins are shown in square brackets). We do not present a comparison with analogous cross sections for the fixed true energy intervals, because they are very similar to those in Fig. 26 . The values $E_{\bar{v}_{\mu}}^{\mathrm{QE}}$ and $Q_{\mathrm{QE}}^{2}$ are defined by Eqs. (13) with $E_{b}=30 \mathrm{MeV}$. The quantitative comparison is given in Table 11. An important remark should be made regarding these data. The covariance matrices reported by the MINER $v$ A Collaboration for the $d^{2} \sigma / d Q_{\mathrm{QE}}^{2} d E_{\bar{v}_{\mu}}^{\mathrm{QE}}$ and $d^{2} \sigma / d Q_{\mathrm{QE}}^{2} d E_{\bar{v}_{\mu}}^{\text {True }}$ cross sections are not positive-definite. ${ }^{4}$ Detailed investigation revealed that the incorrect covariances are located in the matrix elements that take into account the correlations between the $Q_{\mathrm{QE}}^{2}$ bins at fixed antineutrino energy bins (for both $E_{\bar{v}_{\mu}}^{\mathrm{QE}}$ and $E_{\bar{v}_{\mu}}^{\text {True }}$ cases), namely - in the elements outside the eight maindiagonal blocks. So we decided to neglect the doubtful correlations and simplify the standard and log-normal covariance matrices by putting the elements of the corresponding off-diagonal submatrices equal to zero. The "scrubbed" covariance matrices are therefore the partitioned matrices containing only six $10 \times 10$ and two $8 \times 8$ main-diagonal blocks. These matrices are positive-definite, while account only a part of correlations. Therefore, the estimations listed in Table 11 should be treated with caution. Keeping this in mind, we may conclude that all the models satisfactory describe this data subset. It is seen that the SuSAM* model has the least standard and log-normal $\chi^{2} \mathrm{~s}$ for the CCQE cross section $d^{2} \sigma / d Q_{\mathrm{QE}}^{2} d E_{v}^{\mathrm{QE}}$. As for other models, the G18 tunes seem better for the CCQE dataset, but for CCQE-like data it is hard to pick out the best one.

\section{Conclusions}

In this paper, we suggest the phenomenological notion of running (energy-dependent) axial-vector mass, $M_{A}^{\text {run }}\left(E_{v}\right)$, as a flexible tool for description of the quasielastic interactions of neutrinos and antineutrinos with nuclei within the framework of the Smith-Moniz RFG model. This intention was inspired by the heuristic fact that the effective dipole axial-vector mass of the nucleon extracted (within the RFG model) in several recent experiments on the CCQE and CCQE-like $v_{\mu}$ and $\bar{v}_{\mu}$ interactions with carbon-rich targets increases with decreasing the mean energy of the $v_{\mu}$ and $\bar{v}_{\mu}$ beams (see Fig. 1).

The function $M_{A}^{\text {run }}\left(E_{v}\right)$ is defined by only two adjustable parameters, $M_{0}$ and $E_{0}$, independent of $Z$ for $Z \geq 6$. The best-fit values of the parameters were obtained from a global statistical analysis of all available self-consistent CCQE and CCQE-like data for substantial variety of nuclear targets and $v / \bar{v}$ energy spectra (see Sect. 3). It is important that the bestfit value of $M_{0}$ is in very good agreement with the axial mass value extracted from the deuterium data as well as with the results of the former statistical analyses $[4,8]$. The parameter $M_{0}$ can be therefore treated as the current (dipole) axialvector mass of the nucleon.

The SMRFG $+M_{A}^{\text {run }}$ model has compared with several competing models and extensively tested on large amounts of recent CCQE and CCQE-like data from the experiments

\footnotetext{
${ }^{4}$ In all our calculations, we automatically check the symmetry, nonsingularity, and positive definiteness of all covariance/correlation matrices involved into the analysis.
} 
MiniBooNE, T2K, and MINERvA. In most cases, the model describes the data with a reasonable and in some cases (MiniBooNE) with remarkable accuracy. The biggest disagreement is with the $\mathrm{T} 2 \mathrm{~K} \mathrm{C}_{8} \mathrm{H}_{8}$ distributions over the final-state proton variables [25] which, however, provide the problem for the rival models as well, even after applying the renormalization procedure (see Sect. 5). It is thought that the discrepancies are at least in part due to the incompleteness of the FSI models implemented into the modern neutrino event generators and perhaps with certain difficulties in the analysis of the post-FSI protons in the T2K near detectors. In general, it can be concluded that there is no single contemporary model that could satisfactorily describe all the current data. Moreover, there are indications of some inconsistencies among the modern data, namely, between low-energy data from T2K ND280 (hydrocarbon) and MiniBooNE and between the higher-energy data from MINER $v A$ and NOMAD.

The best-fit values of $M_{0}$ and $E_{0}$ are somewhat sensitive to variations of the input parameters of the SM RFG model (Fermi momenta, separation energies) and of the models for the nucleon electromagnetic form factors. However the fit can almost automatically be repeated with the modifications of the RFG model (e.g., Bodek-Ritchie RFG), or its extensions (SF, LFG, CFG, etc.), as well as with the more advanced nuclear models. A more sophisticated parametrization of the function $M_{A}^{\text {run }}\left(E_{v}\right)$ seems to be unreasonable for the presentday level of accuracy of the CCQE and CCQE-like data but may be needed in the future. Individual parametrizations for different nuclei or nuclear groups are also unreasonable today, but mainly because the currently available dataset for the inorganic heavy nuclear targets is not sufficiently accurate and self-consistent.

There is no statistically significant difference between the $M_{A}^{\text {run }}$ parameters extracted separately from the $v_{\mu}$ and $\bar{v}_{\mu}$ data, but there is a faint hint on possible difference (larger $M_{0}$ and smaller $E_{0}$ for $\bar{v}_{\mu}$ interactions). In any case, the available $\bar{v}_{\mu}$ dataset is not yet sufficient for a more definite statement. To draw more robust conclusions it is desirable to compare the $\mathrm{SMRFG}+M_{A}^{\text {run }}$ model predictions with the very new high-statistics measurements of MINER $v \mathrm{~A}$ [161] (broad-spectrum $v_{\mu}$ beam peaking around $6 \mathrm{GeV}$ ) and T2K ND280/INGRID [157] (off-axis $v_{\mu}$ and $\bar{v}_{\mu}$ ), [158] (off-axis $v_{\mu}$ ), [159] (on-axis, $v_{\mu}$ ), and [160] (combined, $\bar{v}_{\mu}$ and $\bar{v}_{\mu}+$ $v_{\mu}$ ) on hydrocarbon and water targets. For a further tune of the model, it would be instructive to add the modern data into the global fit.

Acknowledgements We thank to Jose Amaro, Stephen Dolan, Ulrich Mosel, and Ignacio Luis Ruiz Simó for providing us with the scripts and/or tabulated results of calculations based on their models. We are very grateful to Samoil Bilenky, Arie Bodek, Askhat Gazizov, Kendall Mahn, Dmitry Naumov, Alexander Olshevskiy, Olga Petrova, Oleg Samoylov, Victor Shamanov, Oleg Teryaev, and our colleagues from the GENIE Collaboration, for helpful discussions and critical comments. We appreciate the assistance of JINR cloud team with cloud infrastructure particularly Nikita Balashov, Alexander Baranov, and Nikolay Kutovskiy. Research of I. K. has been supported by the Russian Science Foundation Grant no. 18-12-00271.

Data Availability Statement This manuscript has no associated data or the data will not be deposited. [Authors' comment: All data generated or analysed during this study are included in this published article. Additional data are available from the corresponding author on reasonable request.]

Open Access This article is licensed under a Creative Commons Attribution 4.0 International License, which permits use, sharing, adaptation, distribution and reproduction in any medium or format, as long as you give appropriate credit to the original author(s) and the source, provide a link to the Creative Commons licence, and indicate if changes were made. The images or other third party material in this article are included in the article's Creative Commons licence, unless indicated otherwise in a credit line to the material. If material is not included in the article's Creative Commons licence and your intended use is not permitted by statutory regulation or exceeds the permitted use, you will need to obtain permission directly from the copyright holder. To view a copy of this licence, visit http://creativecomm ons.org/licenses/by/4.0/.

Funded by $\mathrm{SCOAP}^{3}$.

\section{Appendix A: Including “mixed" data into $\chi^{2}$ sum}

The expression (3) for $\chi^{2}$ becomes a bit more complicated when some data represent a combination (for example, sum, as in the IHEP-ITEP experiment [139]) of quantities measured in experiments with neutrino and antineutrino beams. Here we consider the simple case when one has to analyze the three uncorrelated datasets $-\mathbf{E}_{1}, \mathbf{E}_{2}$, and $\mathbf{E}_{3}=\mathbf{E}_{1}^{\prime}+\mathbf{E}_{2}^{\prime}$, where the pairs $\left(\mathbf{E}_{1}, \mathbf{E}_{1}^{\prime}\right)$ and $\left(\mathbf{E}_{2}, \mathbf{E}_{2}^{\prime}\right)$ have the common normalization uncertainties. Let us construct the sum for minimization in this case:

$$
\begin{aligned}
\chi_{\text {mix }}^{2}= & \sum_{k=1,2}\left\{\sum_{j \in G_{k}} \frac{\left[N_{k} T_{k j}(\lambda)-E_{k j}\right]^{2}}{\sigma_{k j}^{2}}+\frac{\left(N_{k}-1\right)^{2}}{\delta_{k}^{2}}\right\} \\
& +\sum_{j \in G_{3}} \frac{\left[N_{1} T_{1 j}^{\prime}(\lambda)+N_{2} T_{2 j}^{\prime}(\lambda)-E_{3 j}\right]^{2}}{\sigma_{3 j}^{2}}
\end{aligned}
$$

(the notation is similar to that in the main text). From the minimization conditions

$\frac{\partial \chi_{\operatorname{mix}}^{2}}{\partial N_{1}}=\frac{\partial \chi_{\operatorname{mix}}^{2}}{\partial N_{2}}=0$

we obtain the expressions for the normalization factors:

$N_{1}=\frac{C_{1} A_{22}-C_{2} A_{12}}{D}, \quad N_{2}=\frac{C_{2} A_{11}-C_{1} A_{12}}{D}$, 
where

$$
\begin{aligned}
A_{12} & =\sum_{j \in G_{3}} \frac{T_{1 j}^{\prime}(\lambda) T_{2 j}^{\prime}(\lambda)}{\sigma_{3 j}^{2}}, \\
A_{k k} & =\sum_{j \in G_{k}} \frac{T_{k j}^{2}(\lambda)}{\sigma_{k j}^{2}}+\sum_{j \in G_{3}} \frac{T_{k j}^{\prime 2}(\lambda)}{\sigma_{3 j}^{2}}+\frac{1}{\delta_{k}^{2}}, \\
C_{k} & =\sum_{j \in G_{k}} \frac{T_{k j}(\lambda) E_{k j}}{\sigma_{k j}^{2}}+\sum_{j \in G_{3}} \frac{T_{k j}^{\prime}(\lambda) E_{3 j}}{\sigma_{3 j}^{2}}+\frac{1}{\delta_{k}^{2}}, \\
D & =A_{11} A_{22}-A_{12}^{2}, \text { and } k=1,2 .
\end{aligned}
$$

\section{Appendix B: Comparison with earlier data}

In this appendix, we present an extended comparison of the $\mathrm{SMRFG}+M_{A}^{\text {run }}$ model predictions with the accelerator data on CCQE total and differential cross sections and event distributions in $Q^{2}$, published before 2010. We also briefly discuss some technical details regarding our statistical analysis, which were omitted from the main text. Since the modern experiments operate mainly with carbon targets, the expected predictive power of the model for other nuclear targets is based only on the earlier ("golden") dataset used in the global fit of $M_{A}^{\text {run }}$. Below, these data are shown by filled symbols and all others by open symbols. For completeness sake, we also show comparison with the partially outdated but not disapproved data. This compilation is not only of historical interest; it also demonstrates a level of consistency (not always satisfactory) between the data subsamples, as well as stability and conformity of the early and later measurements. Besides, it may be particularly useful to display the remaining data gaps of all kinds (nuclear targets, kinematic ranges) to be filled in future experiments. In all figures, the calculations performed with the best-fit values of the parameters $M_{0}$ and $E_{0}(7)$ are shown as solid curves, the inner and outer shaded bands correspond, respectively, to $1 \sigma$ and $2 \sigma$ deviations from the best-fit. No renormalization is applied. In most cases, the $\mathrm{SMRFG}+M_{A}^{\text {run }}$ model satisfactory describes the earlier CCQE data in the wide energy and $Q^{2}$ ranges, including multi-GeV (anti)neutrino energies out of sight of the current accelerator experiments. The remaining problems with description of the earlier data are mainly related to imperfections of the previous analyses of the inelastic backgrounds, FSI effects, and, for non-deuterium targets and at low energies, to the poorly controlled systematic uncertainty in the discrimination between the CCQE and CCQE-like data samples.

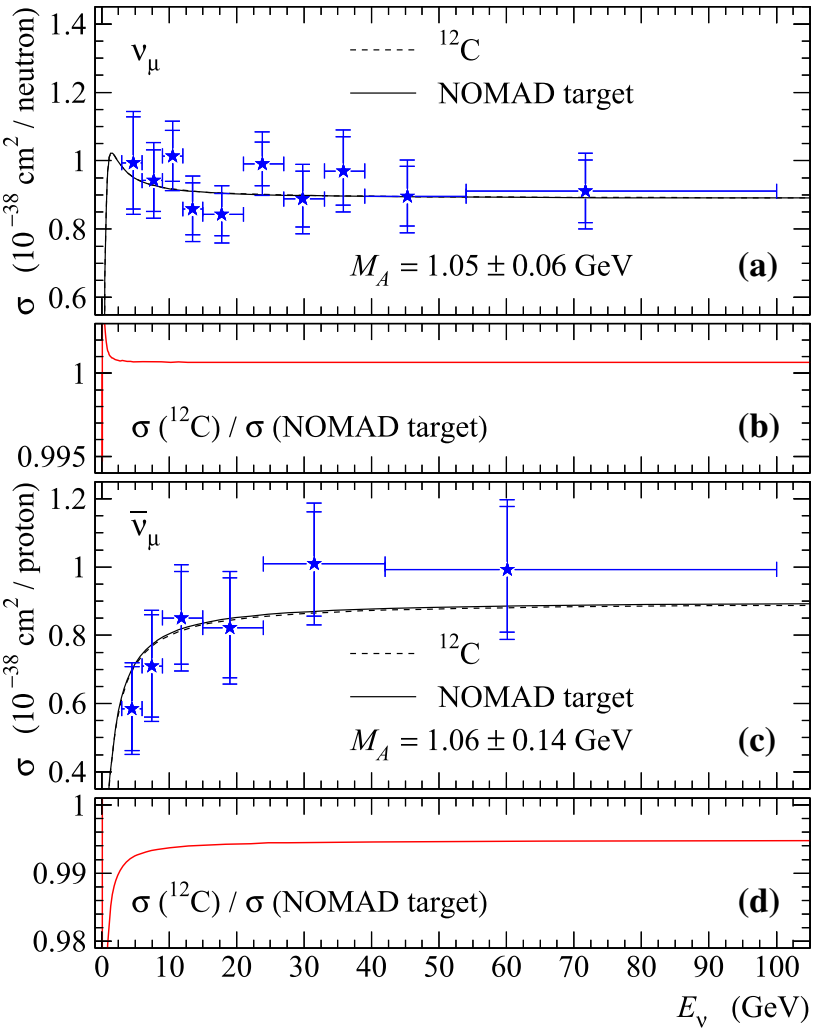

Fig. 27 Total CCQE $v_{\mu}$ and $\bar{v}_{\mu}$ cross sections measured in the NOMAD experiment [34] in comparison with predicted cross sections per bounded nucleons for pure carbon target and NOMAD composite target (panels a and c). The outer and inner error bars indicate total errors and those with subtracted uncertainties due to the (anti)neutrino flux normalization, respectively. Ratios of the cross sections for carbon and NOMAD target are shown in panels $\mathbf{b}$ and $\mathbf{d}$. All calculations are done using the fixed values of the axial masses obtained by the authors of experiment as shown in the legends

\section{B.1 Total CCQE cross sections}

\section{B.1.1 NOMAD}

Considering that the asymptotic behavior of the total CCQE cross sections in the $\mathrm{SMRFG}+M_{A}^{\text {run }}$ model is largely determined by the data of the NOMAD experiment [34] (by adjusting the model parameters to the data), let us dwell on the latter in more detail. Figure 27 displays the total CCQE $v_{\mu}$ and $\bar{v}_{\mu}$ cross sections measured in the NOMAD experiment [34] in comparison with the model predictions for the pure carbon target and for the real NOMAD composite target. Also shown the ratios of the cross sections calculated for the carbon target and NOMAD target. According to Ref. [216] the NOMAD drift chamber consists (by weight) of $64.30 \%$ carbon, $5.14 \%$ hydrogen, $22.13 \%$ oxygen, $5.92 \%$ nitrogen, $0.30 \%$ chlorine, $1.71 \%$ aluminum, $0.27 \%$ silicon, $0.19 \%$ argon, and $0.03 \%$ copper. Calculations show that the $v_{\mu}\left(\bar{v}_{\mu}\right)$ cross sections in the multi-GeV region are fully (almost) insensitive to the 
admixtures to the main carbon component and thus the complexity of the target composition does not provide additional uncertainties. For simplicity, the calculations are done with the current axial masses $M_{A}$ (rather than $M_{A}^{\text {run }}$ ). It is evident that this simplification does not affect the above conclusion. It should be also recorded that the NOMAD results for the cross sections measurement and axial mass extraction are obtained using an extended Gari-Krümpelmann model of the nucleon electromagnetic form factors, "GKex(05)" [217]. The $M_{A}$ extractions performed with the $\operatorname{BBBA}(07)$ form factor model $[4,5]$ increase the axial mass value by about $0.8 \%$ and $0.9 \%$ for, respectively, $v_{\mu}$ and $\bar{v}_{\mu}$ induced reactions. It is pertinent to note here that this is a typical uncertainty in global analyses of $M_{A}$ due to the current uncertainty in our knowledge of electromagnetic form factors.

The new improved analysis of the NOMAD dataset is presented in Ref. [218]. The official results of the NOMAD Collaboration [34] were based on the $v_{\mu}$ CCQE candidates selected from 14, 021 single- and two-tracks events with total selection efficiency and purity of about $34 \%$ and $50 \%$, respectively. The $\bar{v}_{\mu}$ CCQE candidates were selected from 2, 237 single-track events only, with efficiency and purity of about $64 \%$ and $38 \%$, respectively. The new (yet unpublished) result on the CCQE cross sections is based on the analysis of the full kinematic range. It, in particular, uses the $[0,2 \pi]$ range for the muon azimuthal scattering angle, $\phi_{\mu}$, while the previous analysis has been limited by the condition $0 \leq \phi_{\mu} \leq \pi$ (this means that the proton track must lie in the bottom hemisphere). Also the new analysis includes a larger fiducial volume, modified selection criteria for the CCQE events, better understanding of the sources of systematics errors for event reconstruction, improved calibration of background events, and model-independent study of nuclear effects and FSI from a comparison of single- and two-tracks events. The improved analysis of high-purity samples with tighter kinematic cuts obtained 16, 800 total two-track candidates with efficiency (purity) of $25 \%(57 \%)$ and 18, 600 total single-track candidates with efficiency (purity) of 29\% (57\%). As a result, the average total CCQE cross section in the neutrino energy range of $2.5-100 \mathrm{GeV}$ was found to be

$\bar{\sigma}_{v}=\left(0.914 \pm 0.013_{\text {stat }} \pm 0.038_{\text {syst }}\right) \times 10^{-38} \mathrm{~cm}^{2} /$ neutron.

It should be compared with the earlier NOMAD result for the neutrino and antineutrino cross sections averaged over the energy range of $3-100 \mathrm{GeV}$ :

$\bar{\sigma}_{v}=\left(0.92 \pm 0.02_{\text {stat }} \pm 0.06_{\text {syst }}\right) \times 10^{-38} \mathrm{~cm}^{2} /$ neutron,

$\bar{\sigma}_{\bar{v}}=\left(0.81 \pm 0.05_{\text {stat }} \pm 0.09_{\text {syst }}\right) \times 10^{-38} \mathrm{~cm}^{2} /$ proton

As is seen, the new and previous results for the $v_{\mu}$ cross section are in very good agreement, while remaining in tension with the MINER $v \mathrm{~A}$ measurement in the area of inter- section. Needless to say, the cross sections averaged over a wide energy interval (about two orders of magnitude for NOMAD) are rather rough characteristic, especially for the $\bar{v}_{\mu}$ cross section shape. Anyway the data can be compared with the $\mathrm{SMRFG}+M_{A}^{\text {run }}$ predictions

$\bar{\sigma}_{\nu}=0.880_{-0.021(0.025)}^{+0.022(0.026)} \times 10^{-38} \mathrm{~cm}^{2} /$ neutron,
$\bar{\sigma}_{\bar{v}}=0.824_{-0.019(0.023)}^{+0.020(0.024)} \times 10^{-38} \mathrm{~cm}^{2} /$ proton

for the $v_{\mu}$ and $\bar{v}_{\mu}$ reactions, respectively. Here the errors correspond $1 \sigma(2 \sigma)$ deviations from the best-fit values of $M_{0}$ and $E_{0}$ obtained in the global fit (narrow shaded bands in Fig. 28). The averaging was made over the energy range of 2.5-100 GeV. Taking into account the normalization factor of about 1.036 and 1.016 for, respectively, $v_{\mu}$ and $\bar{v}_{\mu}$ (see Appendix Table 13), we may conclude that the $\mathrm{SMRFG}+M_{A}^{\text {run }}$ model is consistent with both earlier and new NOMAD data.

Figure 28 displays the total cross sections (per interacting nucleon) for the CCQE $v_{\mu}$ and $\bar{v}_{\mu}$ scattering on carbonrich detector targets for a wide energy range. Shown are the data of MiniBooNE [13,14,165], SciBooNE [15,16], NOMAD [34], and T2K INGRID [23]. Also shown are the LAr-TPC data point [135] (liquid argon) and the data points from MINER $v$ A $[115,116]$ (hydrocarbon). The MINER $v A$ result has been discussed at length in Sect. 5.3.1. Here we only remind that it was obtained using cuts on the muonmomentum components and muon scattering angle $\left(\theta_{\mu}<\right.$ $20^{\circ}$ ). According to our estimations, these cuts considerably reduce the total CCQE cross section below $\sim 4 \mathrm{GeV}-$ up to about $60 \%$ in the lowest energy bin. So, these data provide only the lower limits for the total CCQE cross section. The estimations also suggest that the MiniBooNE and MINERvA CCQE data are consistent within the errors, but there is an essential tension between the MINER $v$ A and NOMAD data. The experimental data are compared with the predictions of SMRFG + $M_{A}^{\text {run }}$, G18_10b_02_11a, SuSAv2-MEC [65], SuSAM$^{*}$ [193], and NUWRO neutrino generator [207,208] (the latter number is taken from Ref. [165]). All calculations are done for pure carbon. For contrasting purposes, we also show the result of the standard SM RFG calculations made with the best-fit values of the current axial mass, $M_{A}^{\mathrm{RFG}}$, extracted separately from the MiniBooNE doubledifferential CCQE cross section data for $\mathrm{CH}_{2}$ target (shown in Figs. 15 and 16, Sect. 5.1.6) and from the NOMAD total CCQE cross section data (plotted in Fig. 28). These fits were performed separately for $v_{\mu}$ and $\bar{v}_{\mu}$ data subsets. The gray bands around the curves represent the $1 \sigma$ uncertainties due to the errors in determination of $M_{A}^{\mathrm{RFG}}$ and normalization factors $\mathscr{N}$. The corresponding parameters are listed in Appendix Table 12. 


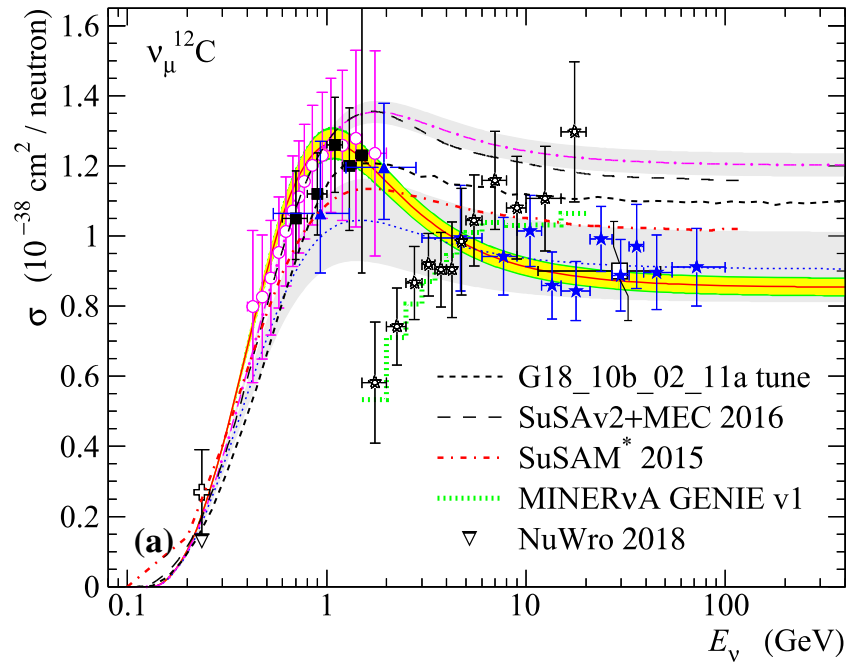

Aguilar-Arevalo et al., FNAL MiniBooNE $2018\left(\mathrm{CH}_{2}\right)$

- Aguilar-Arevalo et al., FNAL MiniBooNE $2010-2013\left(\mathrm{CH}_{2}\right)$

\$ Ruterbories et al., FNAL MINERvA 2019 (Hydrocarbon)

$\triangle$ Patrick et al., FNAL MINERvA 2018 (Hydrocarbon)

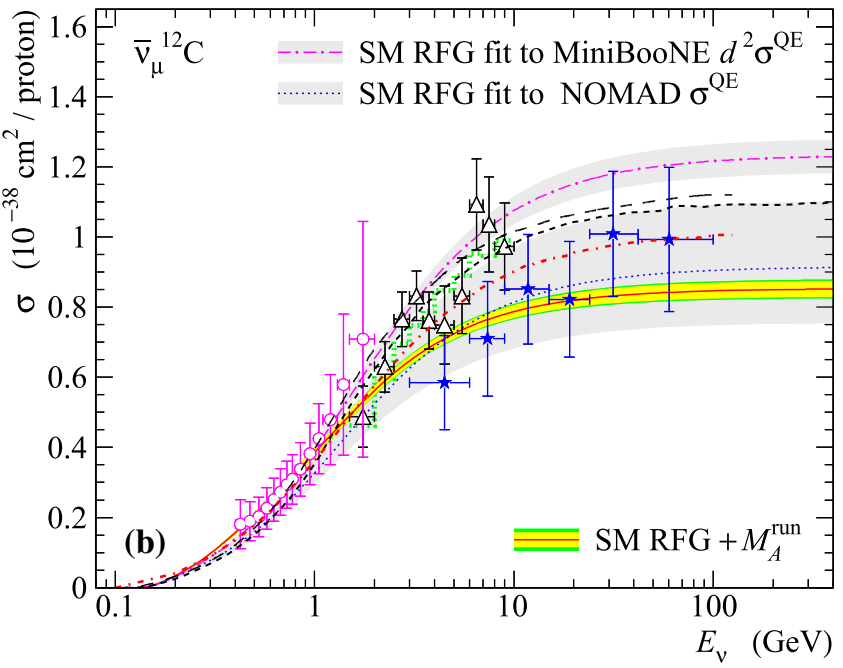

- Aunion et al., FNAL SciBooNE $2010\left(\mathrm{C}_{8} \mathrm{H}_{8}\right)$

$\star$ Lyubushkin et al., CERN NOMAD $2009\left({ }^{12} \mathrm{C}\right)$

$\square$ de la Ossa Romero, CERN LAr TPC $2007\left({ }^{40} \mathrm{Ar}\right)$

- Abe et al., T2K INGRID $2015\left({ }^{12} \mathrm{C}\right)$
Fig. 28 Total CCQE $v_{\mu}$ (a) and $\bar{v}_{\mu}$ (b) cross sections per interacting nucleon for carbon reach targets and argon, as measured in MiniBooNE [13, 14, 165], SciBooNE [15, 16], LAr-TPC [135], NOMAD [34], T2K INGRID [23], and MINER $v A$ [115,116]. The latter are not true total CCQE cross sections, but differential cross ones (vs. $E_{v}^{\mathrm{QE}}$ ) extracted after applying several rigid kinematic cuts, see text; and the dotted histograms represent the respective predictions of the MINER $v$ A GENIE tune v1 $[115,116]$. The vertical error bars represent the total experimental errors including the flux normalization uncertainties. The data points marked with open symbols were not included into the statisti- cal analysis. The solid curves and narrow inner/outer bands correspond to the best-fit values of $M_{0}$ and $E_{0}(7)$ and $1 \sigma / 2 \sigma$ deviations from the best fit. The dashed-dotted and dotted curves represent, respectively, the

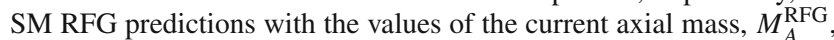
obtained from individual fits to the MiniBooNE double-differential CCQE dataset and to the NOMAD CCQE dataset for $\sigma_{\text {tot }}^{\mathrm{QE}}$. The gray bands indicate the $1 \sigma$ uncertainty of these fits. Other curves represent the predictions by the two superscaling models and G18_10b_02_11a tune. Also shown the NUWRO prediction for the total $\bar{v}_{\mu}^{12} \mathrm{C}$ cross section at $E_{v}=236 \mathrm{MeV}$ (borrowed from Ref. [165])
Table 12 Best-fit values of axial mass, $M_{A}^{\mathrm{RFG}}$, and normalization factors, $\mathscr{N}$, derived from the NOMAD data on CCQE total cross sections [34] and MiniBooNE data on CCQE double-differential cross sections $[13,14]$ using the SM RFG model

\begin{tabular}{lllll}
\hline Dataset & ndf & $M_{A}^{\mathrm{RFG}}(\mathrm{GeV})$ & $\mathscr{N}$ & $\frac{\chi_{\mathscr{N}}^{2}}{\text { ndf- } 2}$ \\
\hline NOMAD $\left(v_{\mu}\right)$ & 10 & $1.07 \pm 0.11$ & $1.000 \pm 0.103$ & 0.68 \\
$\operatorname{NOMAD}\left(\bar{v}_{\mu}\right)$ & 6 & $1.08 \pm 0.19$ & $0.992 \pm 0.137$ & 0.55 \\
MiniBooNE $\left(v_{\mu}\right)$ & 137 & $1.35 \pm 0.03$ & $1.003 \pm 0.013$ & 0.26 \\
MiniBooNE $\left(\bar{v}_{\mu}\right)$ & 75 & $1.38 \pm 0.04$ & $1.016 \pm 0.021$ & 0.48 \\
\hline
\end{tabular}

It is not news that the RFG models cannot describe the total CCQE cross sections at both low (MiniBooNE, SciBooNE, $\mathrm{T} 2 \mathrm{~K}$ ) and high (NOMAD, LAr-TPC) energies, but it is less well known that the same is also true for more sophisticated recent models, namely, the models that account for the MEC contribution are broadly consistent with the low-energy data and with the high-energy MINER $v \mathrm{~A}$ data, but are in conflict with the NOMAD and LAr-TPC measurements, as well as with essentially all earlier high-energy data for other nuclear targets (e.g., IHEP-ITEP 1985 [138, 139]). On the contrary, the SMRFG $+M_{A}^{\text {run }}$ model, being tuned to these data, does not fit the MINER $v$ A measurements. We will return to this problem later. MiniBooNE's measurement of monoenergetic $\mathrm{CC} v_{\mu}$ interactions [165] (point at $236 \mathrm{MeV}$ in panel (a), see also Sect. 5.1.4) is of special interest. Recall that formally it represents the total $v_{\mu}{ }^{12} \mathrm{C} \mathrm{CC}$ cross section, but since the neutrino energy is below the inelastic threshold, it is less model-dependent in comparison to the higher-energy data.

\section{B.1.2 Earlier data}

Figures 29 and 30 show comparison of calculations with the early experimental data on the total cross sections for the CCQE $v_{\mu}$ and $\bar{v}_{\mu}$ scattering on nucleons bound in various nuclei. Figure 29 displays the cross sections on free neutrons and protons extracted from the ANL and CERN measurements on deuterium performed in ANL [94-98] (see also Ref. [146]), and CERN [134], hydrogen (BNL) [79], neonhydrogen mixture (FNAL) [107], freon (IHEP) [80,142], and CERN NOMAD drift chambers used as an active target 

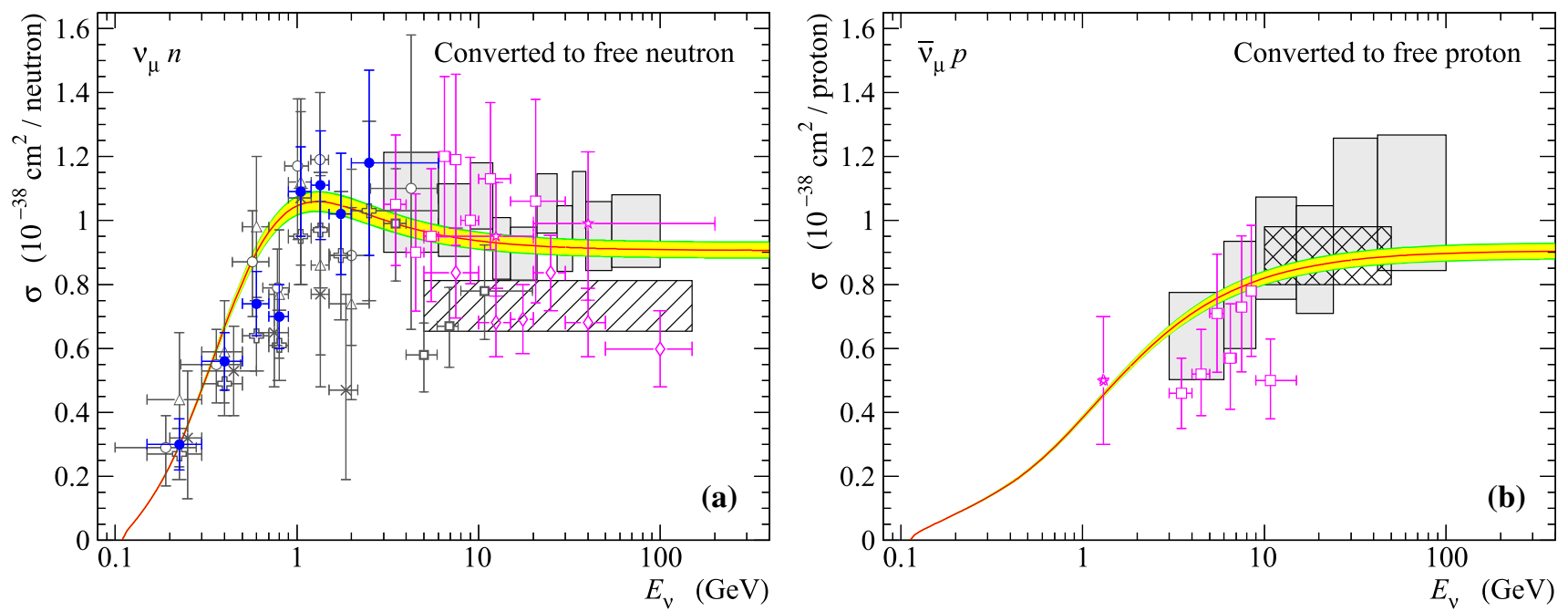

$$
\begin{array}{cl}
* & \text { Mann et al., ANL 1972, } 1973 \text { (D) } \\
\circ & \text { Barish et al., ANL 1975, } 1977 \text { (D) } \\
\lessgtr & \text { Singer et al., ANL } 1977 \text { (D) } \\
\star & \text { Fanourakis et al., BNL } 1980\left(\mathrm{H}_{2}\right) \\
\star & \text { Kitagaki et al., FNAL } 1983 \text { (D) }
\end{array}
$$

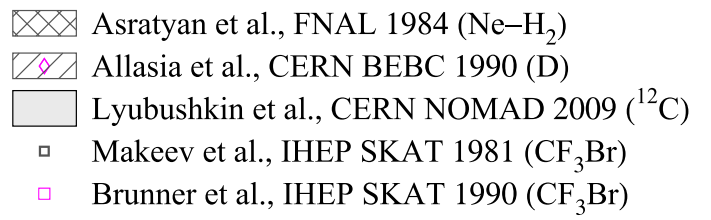

Fig. 29 Total CCQE cross sections for $v_{\mu}$ and $\bar{v}_{\mu}$ scattering on free neutrons (a) and protons (b) extracted from the measurements on deuterium (ANL 1972 [94], 1973 [95], 1975 [96, 146], 1977 [97,98], CERN BEBC 1990 [134], and FNAL 1983 [106]), hydrogen (BNL 1980 [79]), neon-hydrogen mixture (FNAL 1984 [107]), heavy freon (IHEP SKAT 1981 [80] and 1990 [142]), and carbon with small admixtures (NOMAD 2009 [34]). The conversions to the free nucleon targets have been per- formed by the authors of the experiments. The vertical error bars and heights of rectangles represent the total errors including the flux normalization and conversion uncertainties. The solid curves and two shaded confidence bands around them correspond to calculations made with the best-fit values of $M_{A}=M_{0}$ and $1 \sigma$ and $2 \sigma$ deviations from the best-fit curves, respectively and made of nearly isoscalar material $(N: Z \simeq 47.56 \%$ : $52.43 \%, Z / A \simeq 0.52$ ) [34]. The extraction procedures for the three latter datasets are obviously more ambiguous than for the deuterium data; they, in particular, did not take into consideration the MEC contributions. So, taking into account other systematic uncertainties, these data can be considered as qualitative upper limits for the CCQE cross sections on free nucleons. It is seen, however, that the NOMAD neutrino data are in agreement with the deuterium data from ANL (in the overlap region) and FNAL (in the multi-GeV region), but are considerably in excess of the CERN BEBC result [134]. We may therefore again conclude that the NOMAD and essentially all other data shown in Fig. 29 do not support the significant MEC contribution needed according the recent MINER $v$ A measurements discussed in Sect. 5.3.1. The figure also demonstrates the extreme poverty of the experimental data on the CCQE $\bar{v} p$ cross section: in fact, currently we have the only data point from the only direct measurement (BNL) [79] performed more than forty ears ago. From all published data of the ANL 12-ft bubble chamber experiment E412, only the ANL 1977 [97] data are included into the global fits of $M_{A}^{\mathrm{D}}$ and $M_{A}^{\text {run }}$. The authors of the experiment evaluated the flux normalization uncertainty to be $\pm 12 \%$.
However in Ref. [98] it has been reconsidered to be $\pm 15 \%$ in the energy range $0.5-1.5 \mathrm{GeV}$ and $\pm 25 \%$ elsewhere. In the fits, we use this more conservative uncertainty. In the prior ANL publication $[94-96,146]$ the corresponding uncertainty was not reported and thus the errors in Fig. 29 are reproduced as is in the citing articles.

Figure 30 displays the total CCQE $v_{\mu}$ and $\bar{v}_{\mu}$ cross sections on bound nucleons measured in experiments on steel (ANL) [93], iron (FNAL NuTeV) [110], aluminium (IHEPITEP) [136-139], freon (CERN, IHEP) [76,122,123,128, 129,141], and propane-freon mixtures (CERN GGM) [131, $132,164]$. These data are compared with the $S M R F G+M_{A}^{\text {run }}$ model and G18_10b_02_11a predictions. Only the most recent IHEP-ITEP data from Refs. [138,139] are included into the global fits of the running axial mass parameters. The experimental data measured with aluminium and iron targets are combined in single panel, because the nuclear effects for these targets are not essentially different and the predicted cross sections for iron are only slightly lower than for aluminum. It can be seen that the G18 tune predicts the cross sections that are significantly higher than most of the data above $1-2 \mathrm{GeV}$. The only exception provide the highenergy data obtained in the $\mathrm{NuTeV}$ experiment [110]. The 

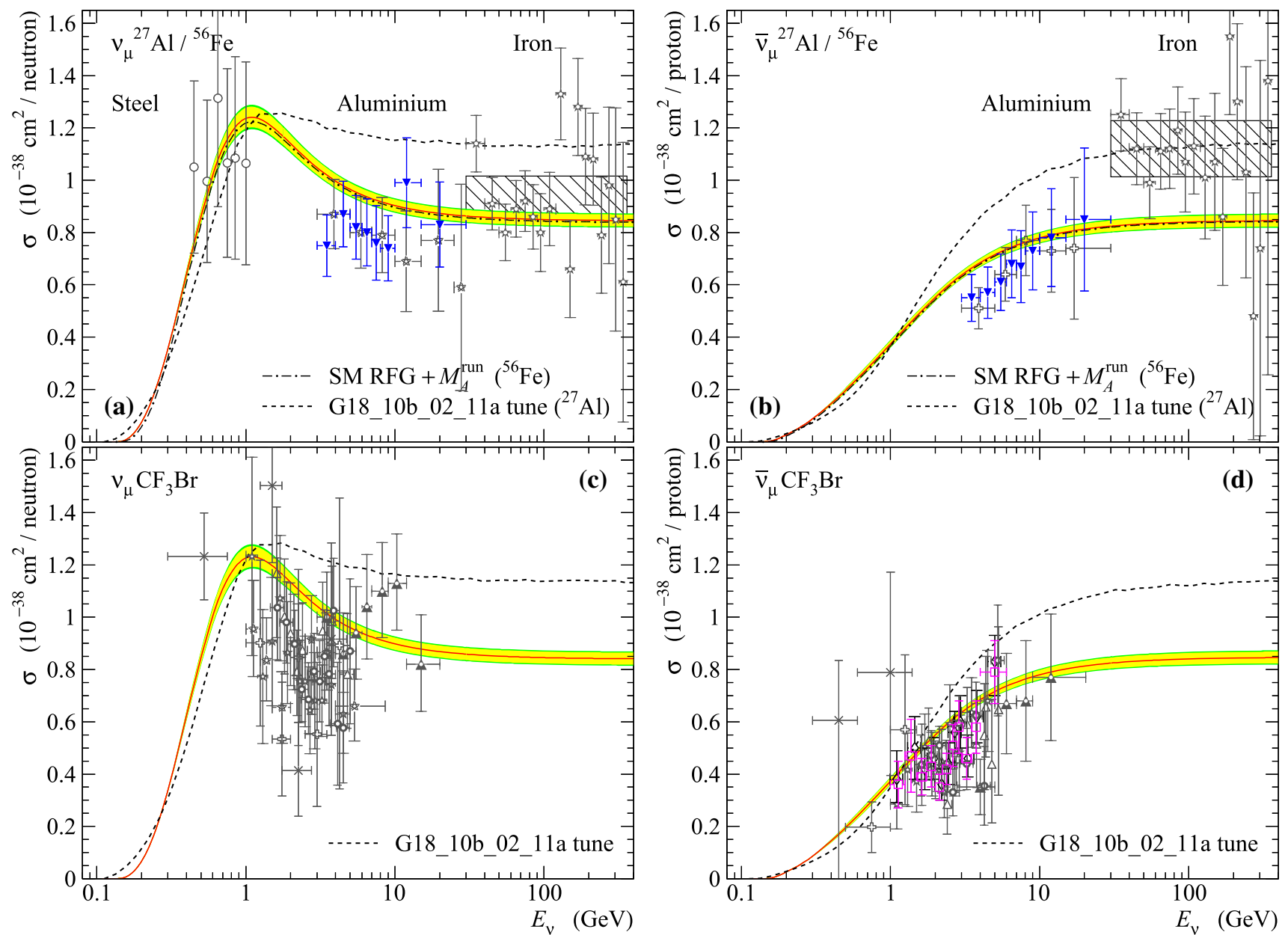

○ Kustom et al., ANL 1969 (Steel)

Suwonjandee, FNAL NuTeV $2004\left({ }^{56} \mathrm{Fe}\right)$

* Franzinetti et al., CERN HLBC $1966\left(\mathrm{CF}_{3} \mathrm{Br}\right)$

↔ Young, CERN HLBC $1967\left(\mathrm{CF}_{3} \mathrm{Br}\right)$

$\star \quad$ Eichten et al., CERN GGM $1973\left(\mathrm{CF}_{3} \mathrm{Br}\right)$

$\triangle$ Rollier et al., CERN GGM $1975\left(\mathrm{CF}_{3} \mathrm{Br}\right)$

- Bonetti et al., CERN GGM $1977\left(\mathrm{CF}_{3} \mathrm{Br}\right)$

$\diamond \quad$ Rollier et al., CERN GGM $1978\left(\mathrm{C}_{3} \mathrm{H}_{8}-\mathrm{CF}_{3} \mathrm{Br}\right)$

$\square \quad$ Armenise et al., CERN GGM $1979\left(\mathrm{C}_{3} \mathrm{H}_{8}-\mathrm{CF}_{3} \mathrm{Br}\right)$

* Pohl et al., CERN GGM $1979\left(\mathrm{C}_{3} \mathrm{H}_{8}-\mathrm{CF}_{3} \mathrm{Br}\right)$

$\Delta \quad$ Grabosch et al., IHEP SKAT $1988\left(\mathrm{CF}_{3} \mathrm{Br}\right)$

$\xi \quad$ Belikov et al., IHEP-ITEP $1981\left({ }^{27} \mathrm{Al}\right)$

* Belikov et al., IHEP-ITEP $1982\left({ }^{27} \mathrm{Al}\right)$

v Belikov et al., IHEP-ITEP $1985\left({ }^{27} \mathrm{Al}\right)$

Fig. 30 Total CCQE cross sections for $v_{\mu}$ and $\bar{v}_{\mu}$ scattering on bound nucleons measured in experiments on steel (ANL 1969 [93]), aluminium (IHEP-ITEP 1981 [136], 1982 [137], 1985 [138,139]), iron (FNAL NuTeV 2004 [110]), trifluorobromomethane (CERN HLBC 1966 [122], 1967 [123], GGM 1973 [76], 1975 [128], 1977 [129], IHEP SKAT 1988 [141], and propane-freon mixture (CERN GGM 1978 [164], 1979 $[131,132])$. The vertical error bars represent the total errors including

data were obtained at record high energies of neutrinos and antineutrinos. This, in particular, means that the CCQE contribution to the total number of events is very small compared to the inelastic contributions, and it is very difficult to unambiguously distinguish the quasielastic events, especially considering that the $\mathrm{NuTeV}$ experiment was carried out with an inactive iron target. For this reason, $\mathrm{NuTeV}$ data the flux normalization and conversion uncertainties. Solid curves represent the $\mathrm{SMRFG}+M_{A}^{\text {run }}$ model predictions; shaded confidence bands around these curves represent $1 \sigma$ and $2 \sigma$ deviations from the bestfitted values of the $M_{A}^{\text {run }}$ parameters. The short-dashed curves represent the cross sections calculated with the G18_10b_02_11a tune. The dashed-dotted curves in panels a and $\mathbf{b}$ represent the cross sections calculated for iron target have a very large scatter and large statistical and systematic errors. We do not include these data in the global fit, following Bodek's recommendation [219], and considering that these results were never approved by the $\mathrm{NuTeV}$ collaboration. 


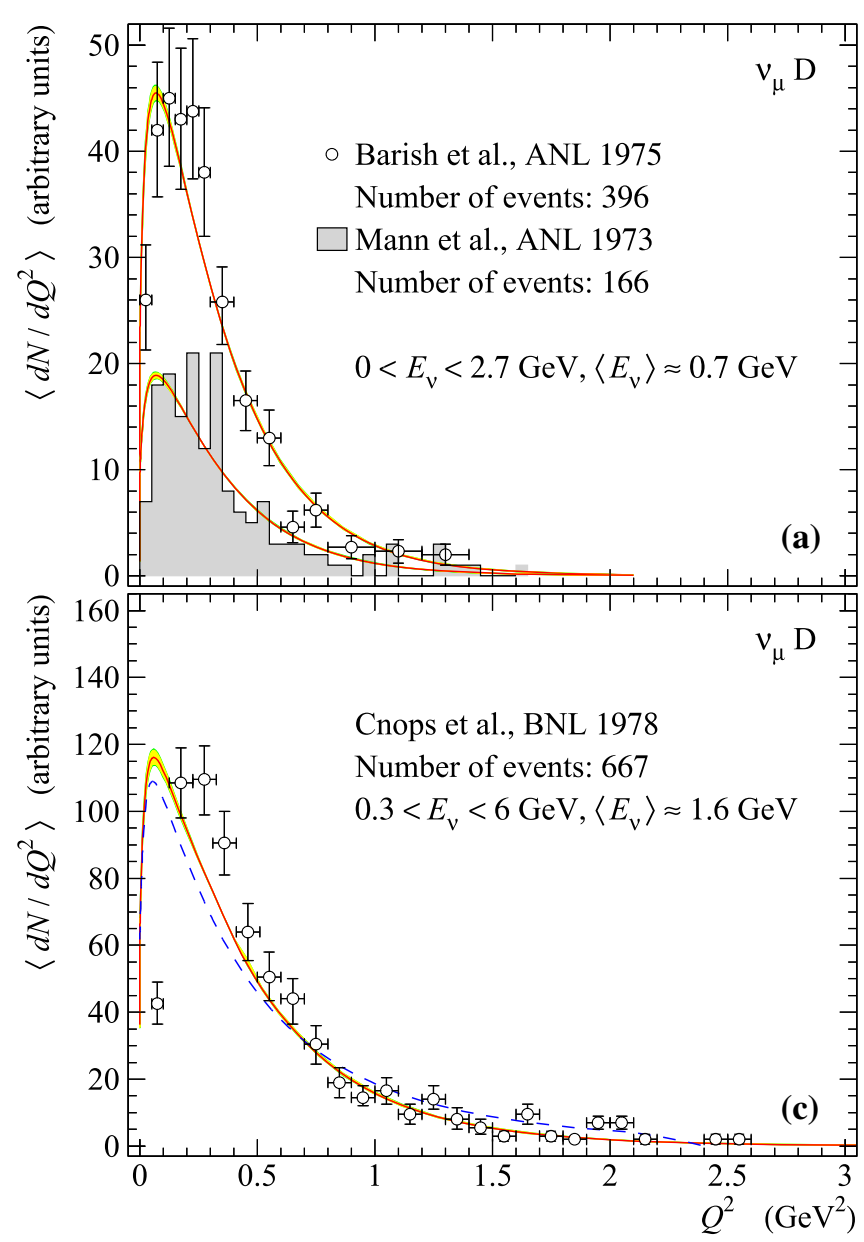

Fig. 31 Flux-weighted $Q^{2}$ distributions for $v_{\mu}$ CCQE reaction measured with ANL and BNL deuterium-filled bubble chambers. Vertical error bars represent the total experimental errors including the $v_{\mu}$ flux normalization uncertainties. The predicted distributions are normalized to the data. Bubble chamber in the experiments ANL 1973 [95] (open circles) and ANL 1975 [146] (light gray histogram) were exposed to the $v_{\mu}$ beam described in Ref. [96] (panel a). The bubble chambers in

\section{B.2 Differential cross sections and distributions}

Figures 31, 32, 33, 34 and 35 show the differential cross sections $d \sigma / d Q^{2}$ and $d \sigma / d y$, and also the $Q^{2}$ distributions predicted with the $\mathrm{SM} \mathrm{RFG}+M_{A}^{\mathrm{run}}$ model in comparison with large experimental $v_{\mu}$ and $\bar{v}_{\mu}$ datasets and very meager $v_{e}$ dataset for the CCQE scattering on bound nucleons. Only a part of these data is presented in Fig. 6 The data under consideration were obtained using single-element detector targets consist of hydrogen [79], deuterium [95,97,99-101,104$106,134,146,220,222]$, aluminum [136, 137,139], and more complex targets such as neon-hydrogen mixture [107,108], propane [126], freon [120,122,123,127,128,128-130,141, $146,152,153,223,224]$, and various propane-freon mixtures $[102,103,131,132,164]$. The cross sections and $Q^{2}$ distributions shown in Figs. 31, 32, 33 and 34 are grouped by

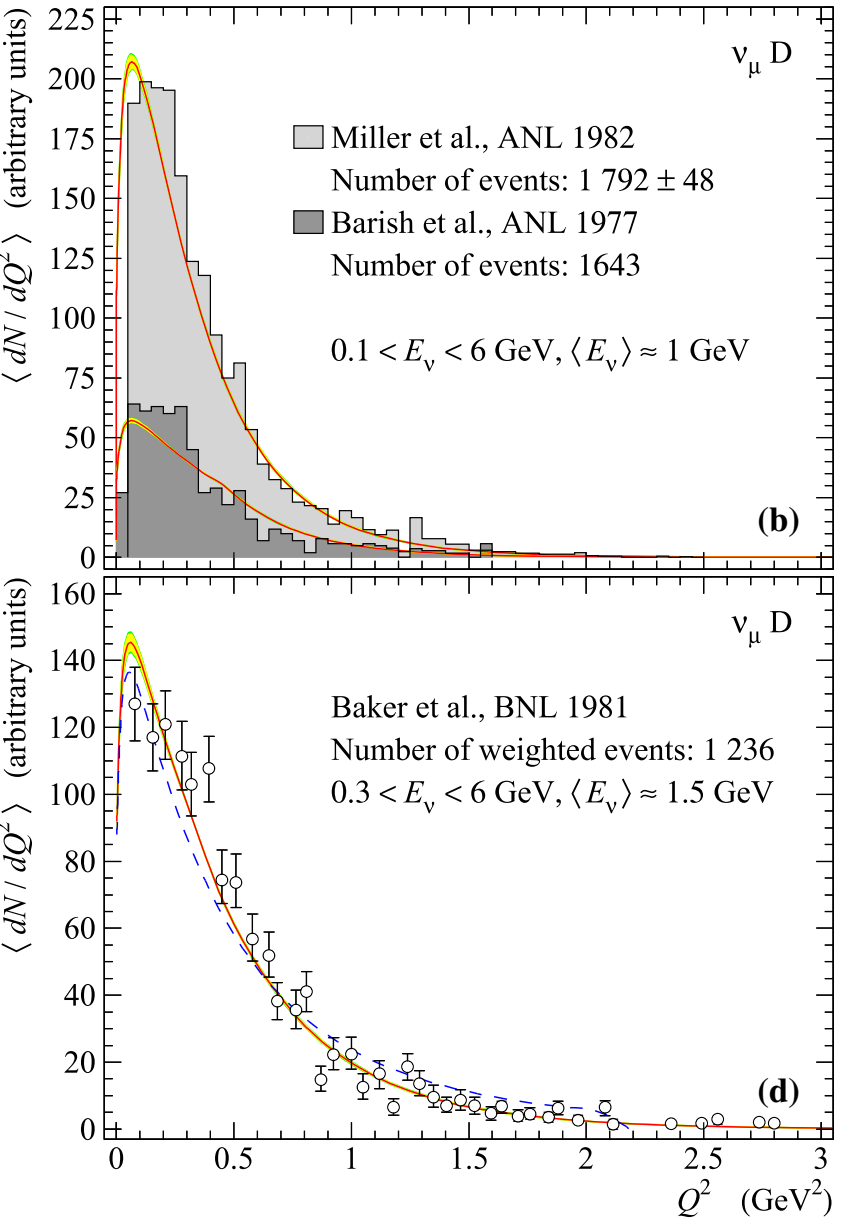

the experiments ANL 1977 [97] (dark gray histogram) and ANL 1982 [99] (light gray histogram) were exposed to the $v_{\mu}$ beam described in Ref. [99] (panel b). The chambers in the BNL 1978 and 1981 experiments were exposed to the $v_{\mu}$ beam described in Ref. [101] (panels $\mathbf{c}$ and $\mathbf{d}$ ). Dashed curves in panels $\mathbf{c}$ and $\mathbf{d}$ represent the $Q^{2}$ distributions calculated at the mean neutrino energies (see text for explanation)

detector targets, the data in Fig. 36 collect the CERN GGM experiments with freon and propane-freone targets, Fig. 37 shows the data on IHEP-ITEP measurements on aluminum target and FNAL measurement on neon-hydrogent target. The legends in the figures list experiment name, reaction type, target, number of events involved into the analysis of the given measured quantity, (anti)neutrino energy range, and estimated mean energy. The area normalized $Q^{2}$ distributions are shown in arbitrary units.

The "golden data" are from the following experiments: BNL 1980 [79], 1990 [104,105,222], FNAL 1983 [106], CERN BEBC 1990 [134] (see panels (a), (b), and (d) of Fig. 32), FNAL 1984 [107] (see panel (d) of Fig. 37), CERN GGM 1979 [132] (panel (d) of Fig. 33), IHEP SKAT 1990 [142] (panels (b) and (d) of Fig. 34), IHEP-ITEP 1985 [139] (panel (c) of Fig. 37). The data presented in other figures are 


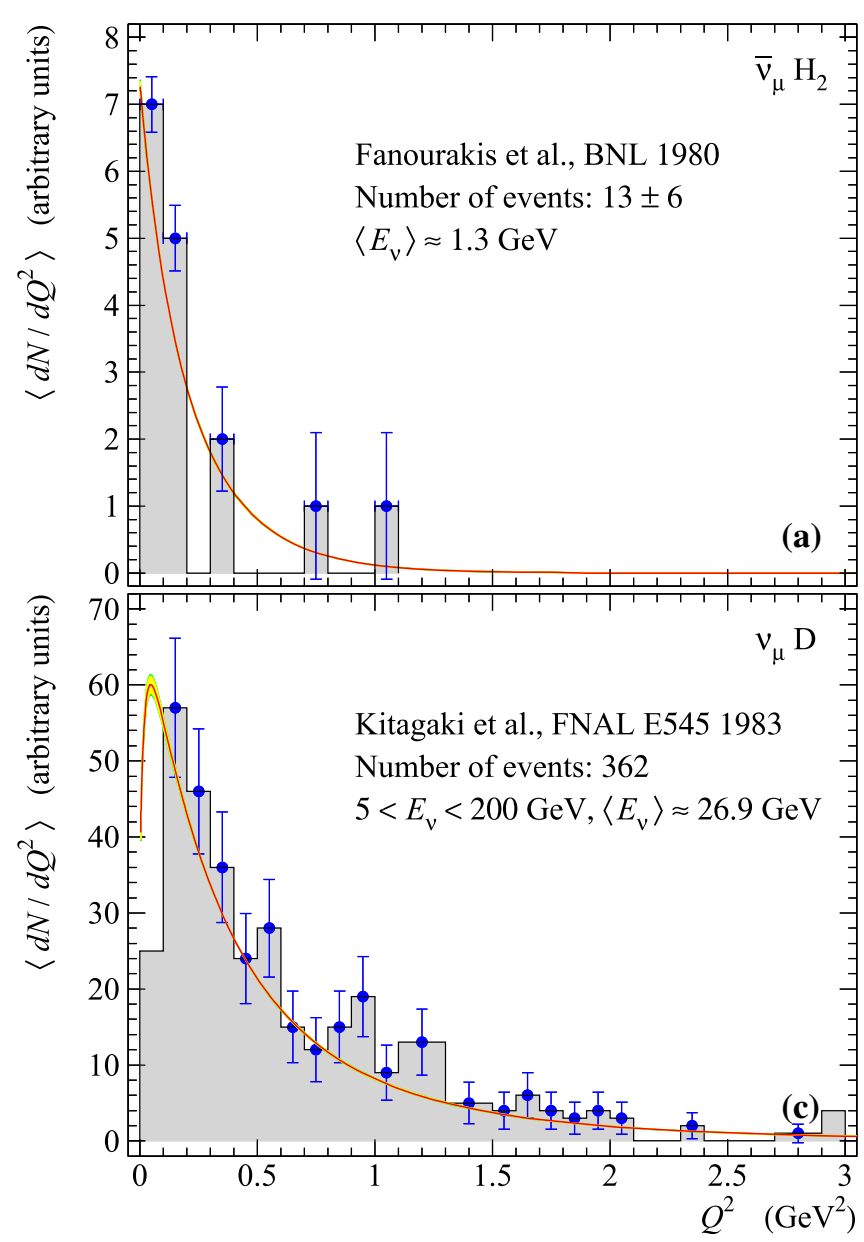

Fig. 32 Flux-weighted $Q^{2}$ distributions and cross section measured in the BNL, FNAL, and CERN experiments. Panel a shows distribution for $\bar{v}_{\mu} \mathrm{CCQE}$ reaction measured with the BNL 7-ft hydrogen-filled bubble chamber exposed to the $\bar{v}_{\mu}$ broad-band, horn-focused beam from the $30 \mathrm{GeV}$ proton beam at the Brookhaven Alternating Gradient Synchrotron (AGS) [79]. The curve is computed using the mean antineutrino energy of $1.3 \mathrm{GeV}$. Panel b shows the $Q^{2}$ distributions for $v_{\mu} \mathrm{CCQE}$ reaction measured in the BNL experiment with the 7-ft deuterium-filled bubble chamber exposed to the BNL wide-band neutrino beam at AGS [104] (open squares). Also shown (filled circles) the result of a later reanalysis of the BNL 1990 data sample described in Ref. [105]. The highest $Q^{2}$ data points shown by open circles are excluded from the global fits since the ordinates of these points could not be reliably digitized from the available versions of the paper [105]. The very first point from that dataset (shown by open circle) is also excluded from the fit because it spoils the correlation matrix. Panel c shows the $Q^{2}$

not involved into the fits and are collected here for comparison only.

Figures 31 and 32 show the flux-weighted $Q^{2}$ distribution for $v_{\mu}$ CCQE reaction measured by ANL, BNL, and FNAL detectors with deuterium targets. We estimate the flux uncertainty in the ANL experiments to be $\pm 15 \%$ except for the highest energies, where the lack of measurements of $K^{+}$ production forces us to assign uncertainty to $\pm 25 \%$. Analysis of the BNL experiment [100] is based on 569,000 neutrino

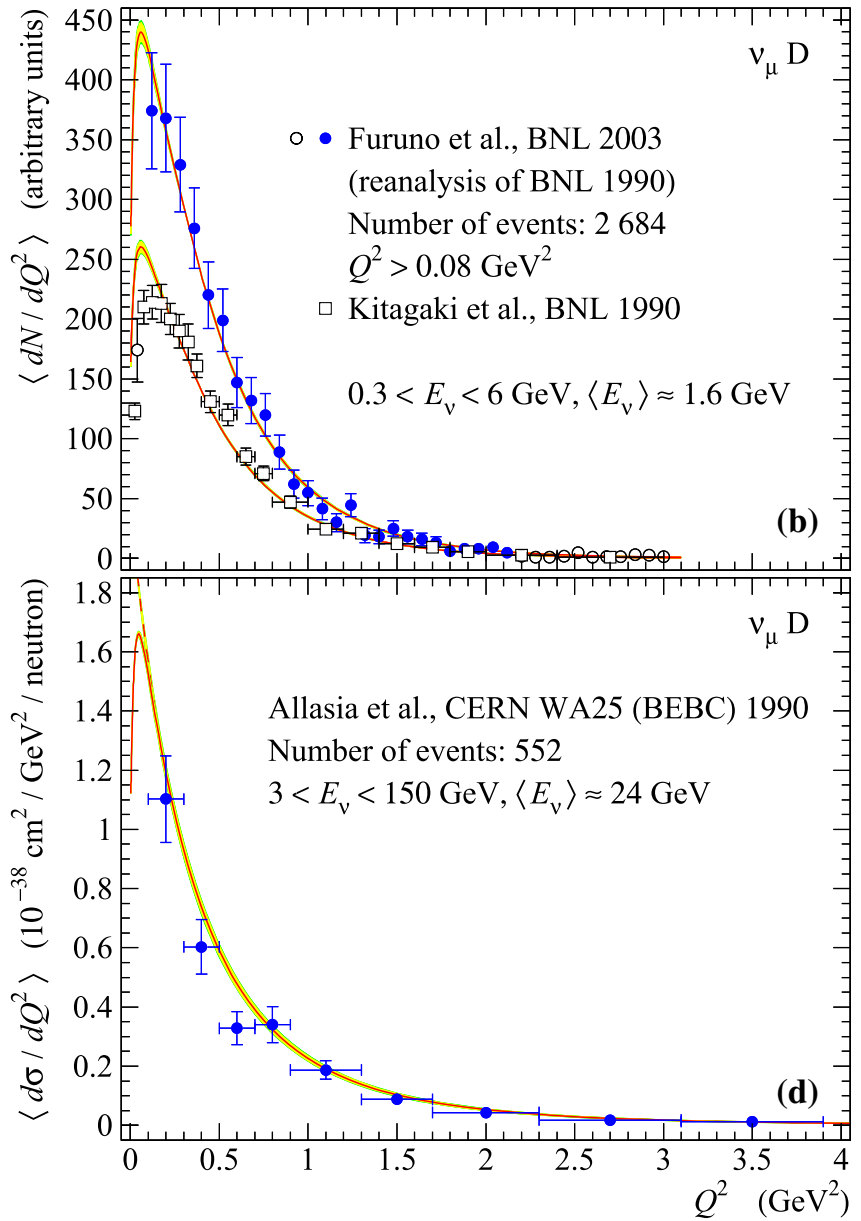

distribution for $v_{\mu}$ CCQE reaction measured with the Fermilab $15-\mathrm{ft}$ deuterium-filled bubble chamber [106]. The curve is computed at the mean antineutrino energy of $26.9 \mathrm{GeV}$. The points and histograms in panels a and $\mathbf{c}$ represent the same datasets and vertical error bars in these panels are purely statistical; the error bars shown in panels $\mathbf{b}$ and d represent the total experimental errors including the neutrino flux normalization uncertainties. Panel $\mathbf{d}$ shows the flux-weighted differential cross section (per neutron) for $v_{\mu}$ CCQE reaction as measured in the CERN WA25 experiment with the Big European Bubble Chamber (BEBC) filled with deuterium and exposed to the high-energy $v_{\mu}$ beam at the CERN Super Proton Synchrotron (SPS) [134] Solid and dashed lines in this panel represent the distributions calculated for the neutron bounded in deuterium and for the bare neutron, respectively. In our calculation, the experimental $v_{\mu}$ energy spectrum was borrowed from Ref. [220]

pictures taken in the BNL 7-fit bubble chamber filled with $\mathrm{D}_{2}$ and an additional 204,000 pictures in $\mathrm{H}_{2}$ and thus represented as study of "neutrino-deuterium" reactions. In panel (c) of Fig. 31 we show the predicted $Q^{2}$ distribution calculated for the $v_{\mu} \mathrm{D}$ reaction with no $v_{\mu} \mathrm{H}_{2}$ contribution. It should be recorded that the neutrino spectra from the BNL wide-band $v_{\mu}$ beam used for the processing of the data by the BNL 1978 experiment [100] is not known. For our calculations we use the $v_{\mu}$ spectrum from Ref. [101]. The flux averaging of the 


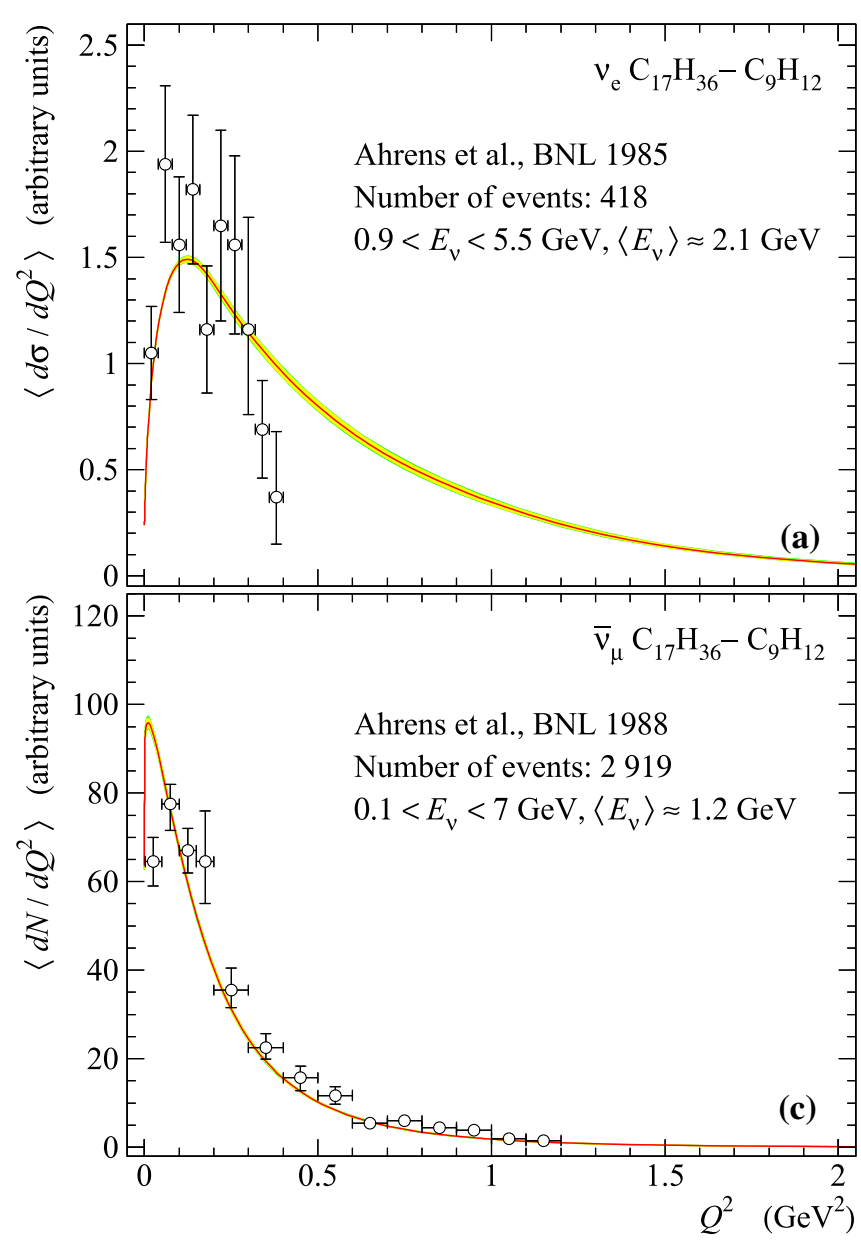

Fig. 33 Flux-weighted differential cross sections per neutron and $Q^{2}$ distributions for $v_{e}$ (panel a), $v_{\mu}$ (panel b) and $\bar{v}_{\mu}$ CCQE reactions measured at BNL $[102,103]$ and CERN [132]. Vertical error bars represent the total experimental errors including the $v / \bar{v}$ flux normalization uncertainties. The BNL detector was filled with a liquid scintillator and exposed to the Brookhaven wide-band $v_{e} / v_{\mu}$ beams at AGS [221]. The

predicted $Q^{2}$ distribution for the BNL 1981 experiment [101] is more definite because the neutrino energy distribution is explicitly provided by the authors. For methodological purposes, in panels (c) and (d) of Fig. 31 we show the distributions $d N / d Q^{2}$ calculated at the mean neutrino beam energy $\left(\left\langle E_{v}\right\rangle=1.6\right.$ and $1.5 \mathrm{GeV}$ for, respectively, panels (c) and (d)). It is clearly seen that such calculations are too rough: they do not reproduce the shapes of the measured $Q^{2}$ distributions and break off at the kinematic boundaries (of about 2.4 and $2.2 \mathrm{GeV}^{2}$ corresponding to the mentioned mean energies), much to the left of the real (experimental) endpoints of the distributions. This exercise also demonstrates the importance of knowing the neutrino energy spectrum for accurate analysis. It is probably the wrong spectrum that explains the contradiction between the data and the calculations shown in panel (c).

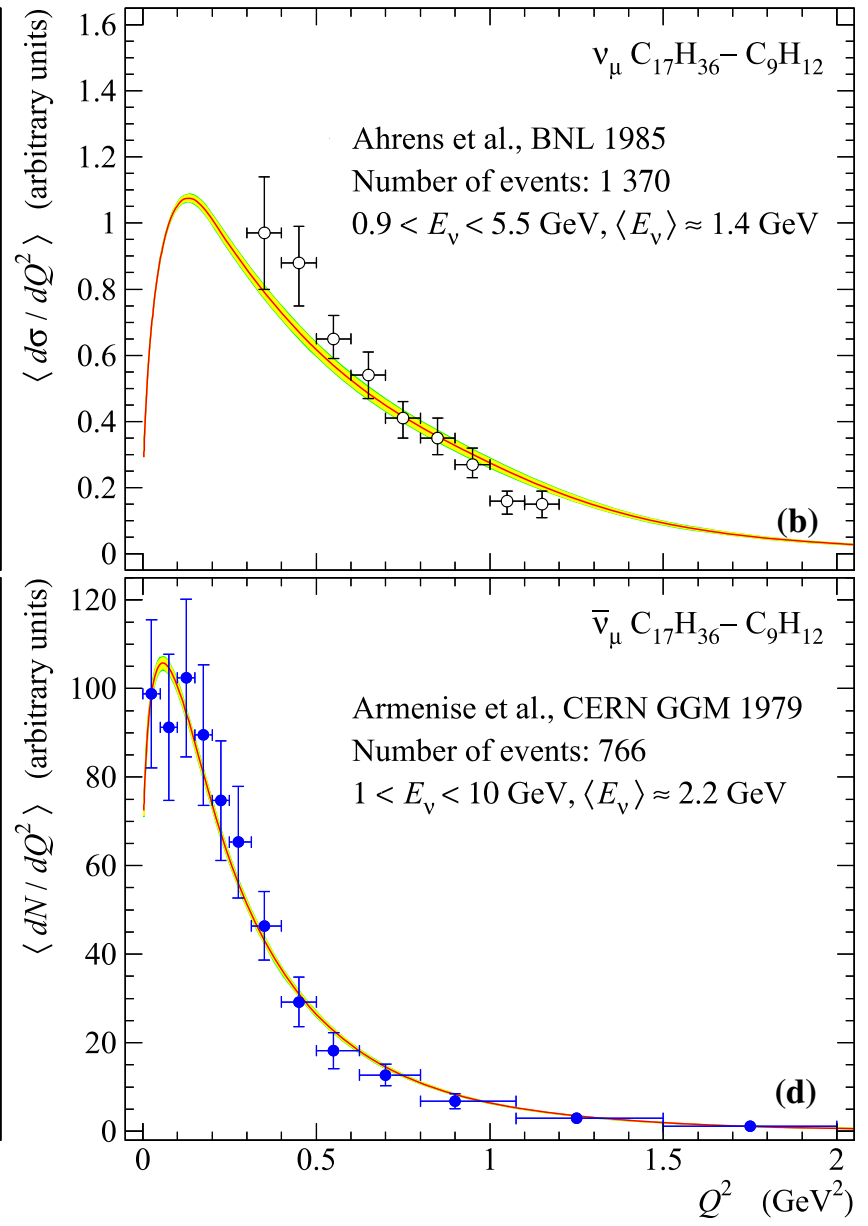

CERN data are obtained with the bubble chamber Gargamelle filled with light propane-freon mixture (87 mole per cent of propane) and exposed to the CERN-PS $\bar{v}_{\mu}$ beam [132]. All the data are given in arbitrary units and the predicted cross sections are normalized to the data

Predicted $Q^{2}$ distributions in comparison with data measured with BNL 1980 [79] and FNAL E545 [106] are calculated for an average antineutrino and neutrino energies because the flux used in the analysis are unknown. The average antineutrino energy in BNL 1980 experiment is estimated to be $1.3 \mathrm{GeV}$, while the energy distribution in the antineutrino beam is peaked at about $1.1 \mathrm{GeV}$. The spectrum was estimated from quasielastic events measured in a previous experiment with the same bubble chamber filled with deuterium (unpublished). Although the number of events measured in the BNL experiment is very small and determined with an error of about $50 \%$, we include this data into the global fit, because this experiment provides a unique dataset for quasielastic antineutrino scattering on pure hydrogen.

In the FNAL 1983 experiment [106], the neutrino flux was obtained from the analysis of the CCQE events using the predicted cross sections with $M_{A}=1.05 \mathrm{GeV}$. In the 


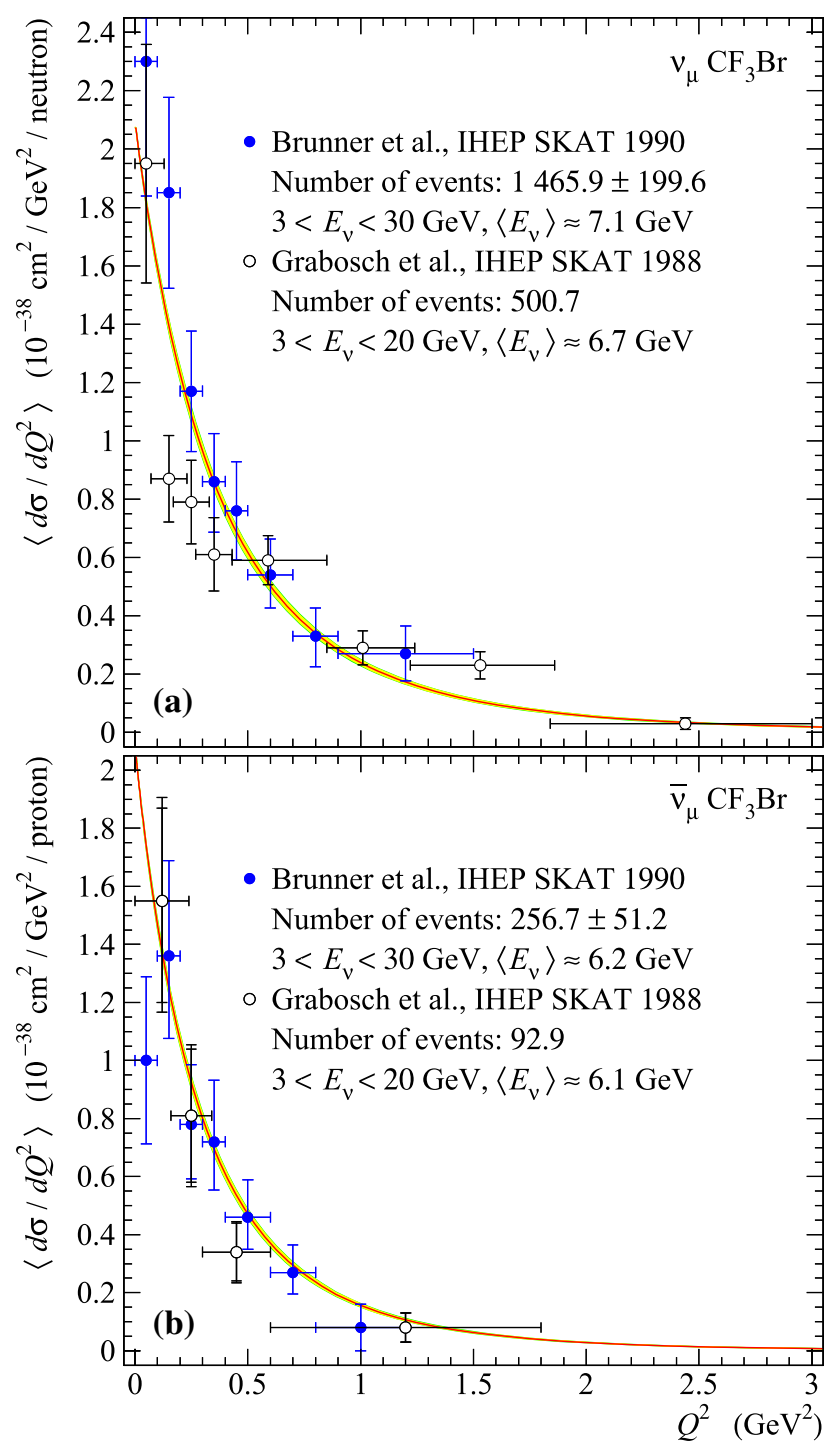

Fig. 34 Flux-weighted differential cross sections per neutron or proton for CCQE $v_{\mu}$ (panels a), $\bar{v}_{\mu}$ (panels b) reactions measured with the freon-filled bubble chamber SKAT exposed to the U70 broad-band $v_{\mu}$ and $\bar{v}_{\mu}$ beams of the Serpukhov proton synchrotron (PS) $[141,142,152]$ (see also Ref. [140] for the preceding analyses of the of the SKAT experiment). Experimental $v_{\mu}$ and $\bar{v}_{\mu}$ energy spectra are borrowed from Ref. [152]. The systematic error includes the uncertainties due to the cross section normalization and nuclear Monte Carlo

energy range below $30 \mathrm{GeV}$ we use the tabulated data for the "effective flux" and above 30 we use the author's Monte Carlo simulation of the flux. By applying this composite flux, we reproduce the average energies at different energy intervals $\left(12.5 \mathrm{GeV}\right.$ for $5<E_{v}<20 \mathrm{GeV}, 39.6 \mathrm{GeV}$ for $20<$ $E_{v}<200 \mathrm{GeV}$, and $23.7 \mathrm{GeV}$ for $5<E_{v}<200 \mathrm{GeV}$ ). The expected uncertainties in the neutrino flux prediction differ significantly for different energies and grow form $\sim 10 \%$ at $14.8 \mathrm{GeV}$ to $\sim 60 \%$ at $200 \mathrm{GeV}$.

The BNL 1990 data [104] shown in Fig. 32 (b) were collected in two periods of runs, in 1976-1977 and 1979-1980,

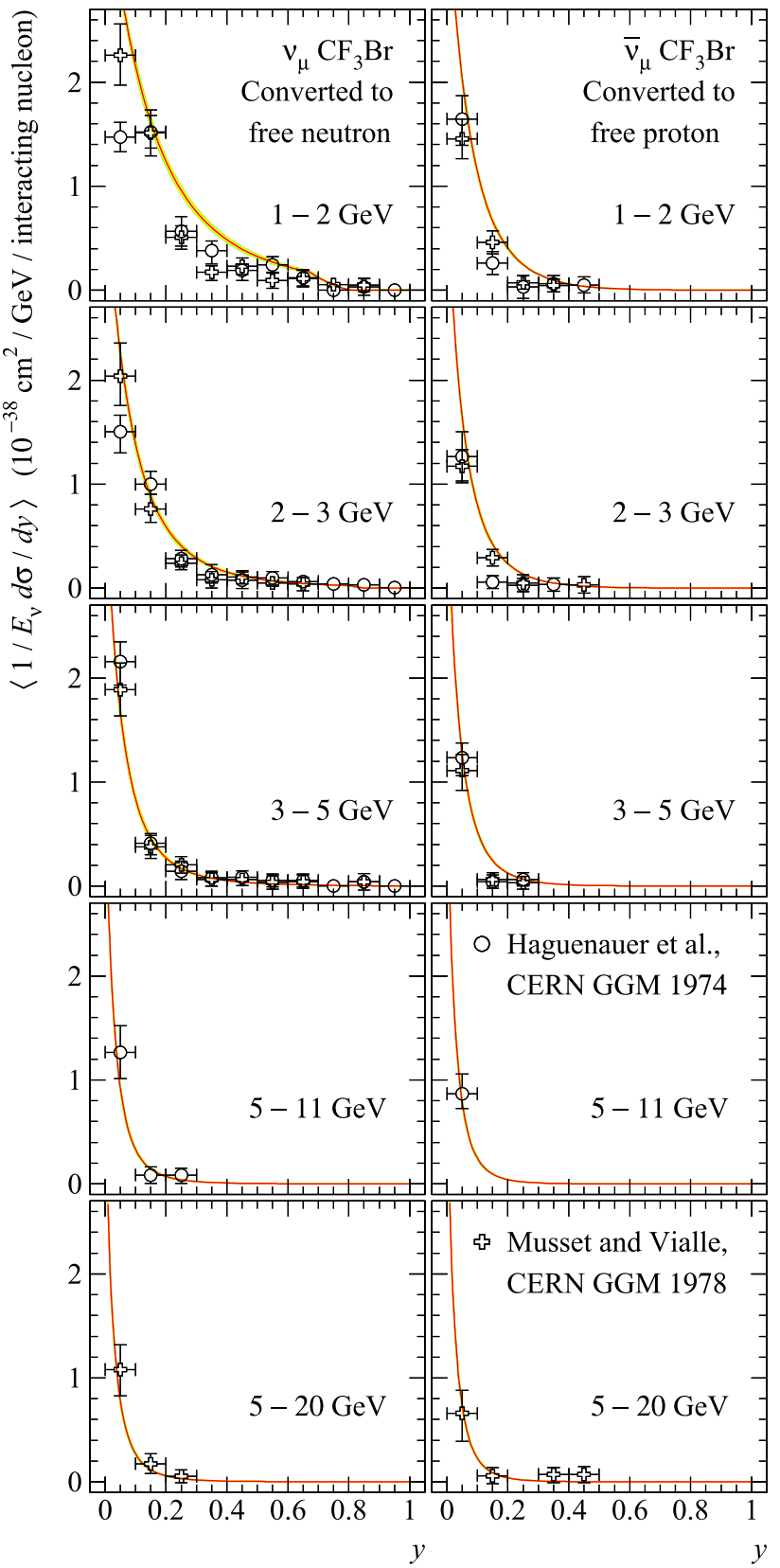

Fig. 35 Flux-weighted differential cross sections divided by energy for $v_{\mu} n$ and $\bar{v}_{\mu} p$ CCQE reactions, measured with the heavy-liquid freonfilled bubble chamber Gargamelle and exposed to the wide-band CERNPS $v_{\mu}$ and $\bar{v}_{\mu}$ beams. The data from Ref. [127] $\left(E_{v}<11 \mathrm{GeV}\right)$ and [130] (all energy intervals) represent two analyses of the same data sample. The measured cross sections were converted to free nucleon target by the authors of the experiment. The quoted error bars include uncertainties in the $v_{\mu} / \bar{v}_{\mu}$ flux and in nuclear Monte Carlo

when the total of $1.8 \times 10^{6}$ pictures were obtained. The result published in Ref. [104] has been reanalysed later [105] (see also Ref. [225]). In the improved analysis, the background to CCQE events has been reevaluated and, as a result, the number of the CCQE events has been increased. The final data sample in the CCQE channel obtained was 2684 and 
the estimated correction to this number (coming from many sources) was found to be $1.06 \pm 0.11$. In this experiment, the neutrino flux was not independently measured and thus the flux independent analysis of Ref. [105] provided the model dependent cross sections. The $\left\langle d N / d Q^{2}\right\rangle$ data set of BNL $1990[104,226]$ for neutrino reactions on deuterium are reliably measured under the condition $Q^{2}>0.08(\mathrm{GeV} / \mathrm{c})^{2}$ (we do not discuss the reasons, but refer to the earlier mentioned original papers). The analysis of Ref. [105] has not been published, but we prefer to use this more recent result in our statistical analysis. At the same time, we are forced to do some rejections. The lowest- $Q^{2}$ data point is excluded from our fits because it strongly contradicts to all model calculation of the $Q^{2}$ distribution. Probable explanation of this discrepancy is the effect of missing recoil proton at very low $Q^{2}$ discussed in the Ref. [104]. Other reasons include a simplified description of the deuteron effects (e.g. neglecting the MEC contribution). Unfortunately, the author's tabulated data for the $Q^{2}$ distribution are not available. Therefore, we used the data taken from the figure in Ref. [105]; the digitization of the data points and, more importantly, associated error bars is extremely unreliable for $Q^{2} \gtrsim 2 \mathrm{GeV}^{2}$. Hence we show these data points for completeness, but exclude them from the global fit. It is seen that the high- $Q^{2}$ data points do not conflict with the predicted distribution.

Let us dwell a little more on the data of CERN BEBC WA25 experiment [134] shown in panel (d) of Fig. 32. In order to demonstrate the magnitude of nuclear effects for these measurements at relatively high energies $\left(\left\langle E_{v}\right\rangle \approx\right.$ $24 \mathrm{GeV})$, we show the predicted $Q^{2}$ distributions calculated for neutron bounded in deuterium nucleus and for the bare neutron. As evident from the comparison of the solid and dashed curves at the lowest $Q^{2}$ bin, the fit to the BEBC WA25 cross sections should be practically insensitive to the nuclear corrections. For the global fit, we slightly modified the dataset of the flux-weighted differential cross section, $\left\langle d \sigma_{v} / d Q^{2}\right\rangle$, for $v_{\mu}$ CCQE reaction per neutron. The full experimental dataset contains 9 bins. It is obvious that the value obtained in the $Q^{2}$ bin $[0.5,0.7] \mathrm{GeV}^{2}$ is not consistent with the values obtained in the neighboring bins that yields unsatisfactory covariance matrix (from MINUIT) and artificial change in the fitted parameters. To avoid this, we combined the data from the two bins $[0.5,0.7]$ and $[0.7,0.9] \mathrm{GeV}^{2}$ into the single one, $[0.5,0.9] \mathrm{GeV}^{2}$; the corresponding systematic error in the combined bin is estimated to be $\pm 10 \%$. It is assumed that the flux uncertainty of $8.6 \%$ is included into the quoted systematic error.

Figure 33 shows the cross sections and $Q^{2}$ distributions measured in the BNL experiment [102,103] with liquid scintillator consisting of $\sim 60 \%$ light mineral oil $\left(\mathrm{C}_{17} \mathrm{H}_{36}\right)$, $\sim 35 \%$ 1,2,4-trimethylbenzene $\left(\mathrm{C}_{9} \mathrm{H}_{12}\right)$, and $\sim 5 \%$ proprietary ingredients and has the $\mathrm{H} / \mathrm{C}$ ratio of about 1.9 [227]. Therefore we use the simplified formula, $60 \%$ of $\mathrm{C}_{17} \mathrm{H}_{36}$
$+40 \%$ of $\mathrm{C}_{9} \mathrm{H}_{12}$, to approximate its chemical composition. The uncertainty of the calculated $v_{e}$ and $v_{\mu}$ spectra from the Brookhaven Alternating Gradient Synchrotron is about $20 \%$ and the related errors for the measured cross sections is about 30\%. In the BNL 1988 experiment were obtained two data sets of event samples so called main and stopping muon samples. Stopping muon sample was used to check the event selection of the main data sample in the low- $Q^{2}$ region and was selected by requiring a single long track stopping in the detector regardless of the existence of a secondary vertex due to a neutron interaction [103]. Panel (c) in Fig. 33 includes both the stopping muon data sample (points at $Q^{2}<0.2$ $\mathrm{GeV}^{2}$ and main data sample (points at $Q^{2}>0.2 \mathrm{GeV}^{2}$ ).

Figure 34 shows the experimental data on the $v_{\mu}$ and $\bar{v}_{\mu}$ differential cross sections obtained with the IHEP SKAT bubble chamber filled with heavy freon and converted to a free nucleon target by the authors of the experiment. We do not show the partially obsolete data of the IHEP SKAT 1981 [80] and 1986 [140] obtained for the slightly different neutrino energy ranges in comparison with the posterior and final (full statistics) SKAT dataset [142]. The earlier SKAT data are consistent with the final result at within the uncertainties and taking into account the difference in the constraints on (anti)neutrino energy used in the analyses. The current axial mass parameter extracted from the measured total and differential cross sections was $1.06 \pm 0.15 \mathrm{GeV}$ for neutrino and $0.71 \pm 0.22 \mathrm{GeV}$ for antineutrino; the analysis used a simple dipole ansatz for the electromagnetic form factor. From Fig. 34 it can be seen that the SKAT data agree with the universal value of $M_{A}=M_{0}=1.008 \mathrm{GeV}$ obtained in our analysis. But it is not the case for total CCQE $v_{\mu}$ and $\bar{v}_{\mu}$ cross sections (see Fig. 29), where agreement with SKAT data is unsatisfactory. Note that this is a common situation for almost all data on the total CCQE cross sections measured using freon-field detectors. It is, of course, mainly related to the outdated analysis of nuclear effects in old experiments.

Experimental data on the slopes of the differential cross sections measured in terms of inelasticity $y=1-E_{\mu} / E_{v}$ are employed for additional test of our best-fit value of the axial mass. Figure 35 shows the flux-weighted slopes $E_{\nu}^{-1} d \sigma / d y$ for the $v_{\mu}$ and $\bar{\nu}_{\mu}$ CCQE reactions as measured using the CERN bubble chamber Gargamelle filled with $\mathrm{CF}_{3} \mathrm{Br}[127,130]$; the data were converted by the authors of experiment to those on free interacting nucleons, taking into account Fermi motion and Pauli suppression. The data from Refs. [127] and [130] represent two different analyses of the same full raw data sample; they partially overlap in the regions below $5 \mathrm{GeV}$ but at higher energies, the two analyses use different bins (5-11 and 5-20 GeV). The analyses are generally consistent with each other and mutually complementary, except the lowest-energy bin and at small inelasticities. Alternative analyses were reported in 


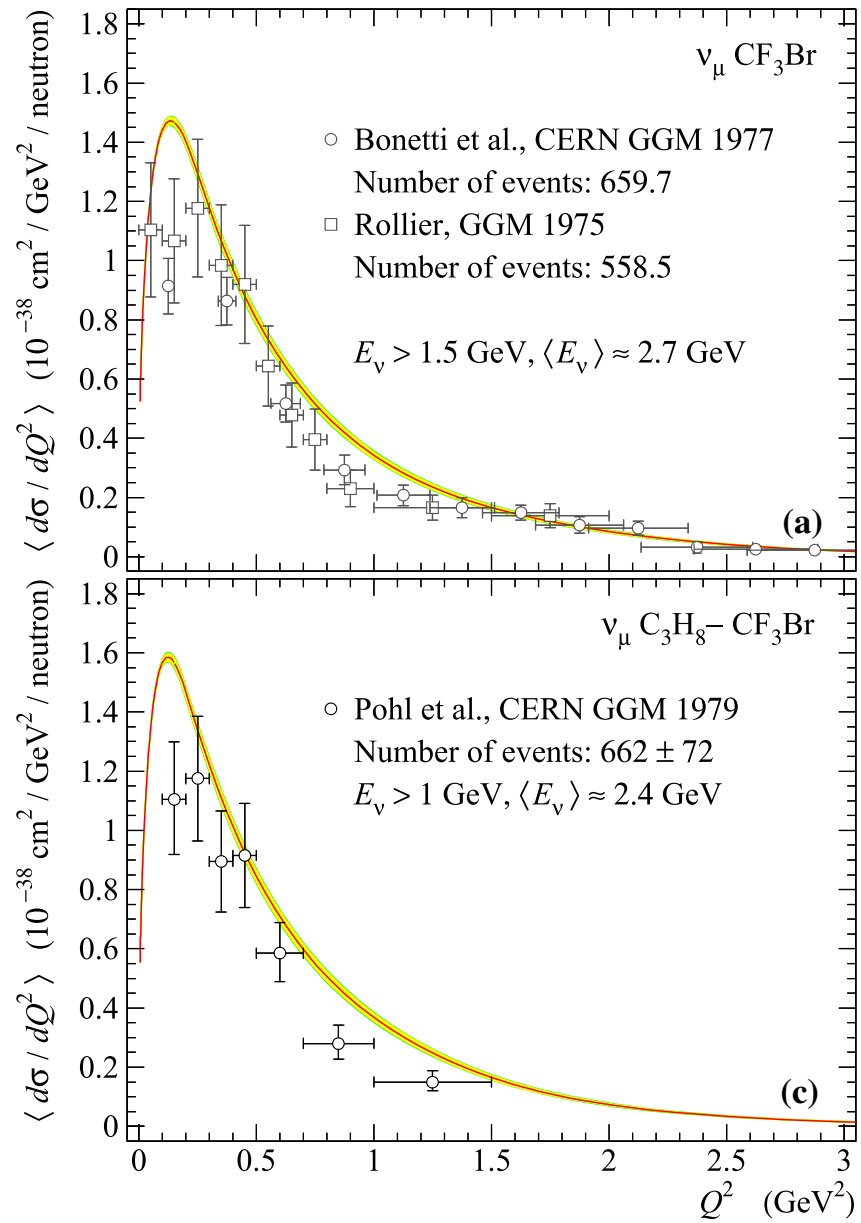

Fig. 36 Flux-weighted differential cross sections for $v_{\mu}$ (panel a) and $\bar{v}_{\mu}$ (panel b) CCQE reactions measured with the heavy-liquid bubble chamber Gargamelle filled with heavy freon and exposed to the CERN-PS $v_{\mu}$ and $\bar{v}_{\mu}$ beams $[129,130]$. Obsolete data measured with CERN GGM $1975[128,146]$ experiment are also shown for completeness. Flux-weighted differential cross sections for $\nu_{\mu}$ (panel c) and $\bar{v}_{\mu}$

Refs. [153,223,224] but they do not provide additional data for comparison.

The predicted cross sections are consistent with the experimental data from four of the five narrow instrumental energy ranges of $2-3,3-5,5-11$, and $5-20 \mathrm{GeV}$. It is also seen that the agreement is generally better for the later analysis [130]. However the predicted $v_{\mu}$ and $\bar{v}_{\mu}$ cross sections in the lowest-energy range $(1-2 \mathrm{GeV})$ systematically overestimate the data. The discrepancy can be partially explained by the outdated nuclear models (including FSI) used in the analyses of the GGM data; as we now know, the MEC contributions and correct FSI modelling are critical in the few-GeV energy region. We cannot recalculate the nuclear effects and use this data sample in the global fit of axial mass, because the required Monte Carlo simulation details are not available.

Another source of the observed disagreement may be associated with the systematics of the (anti)neutrino flux nor-

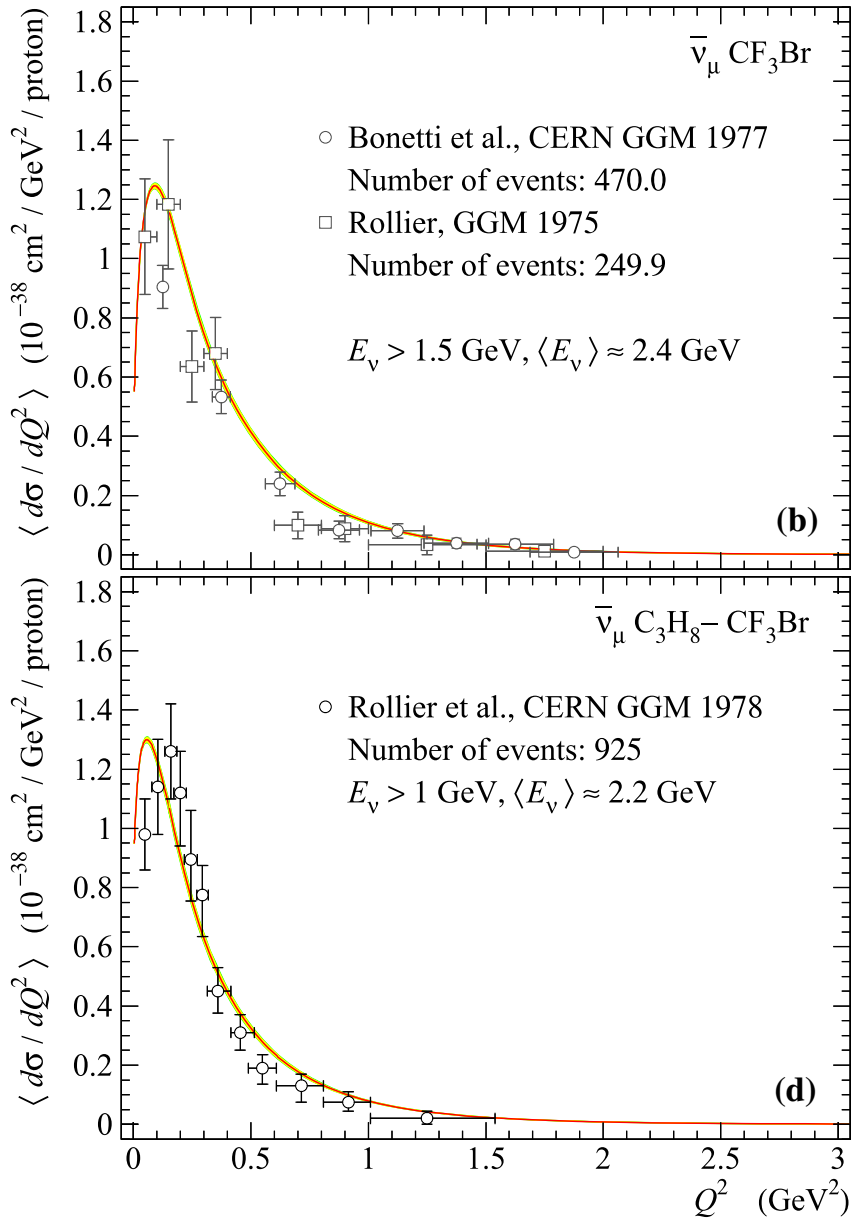

(panel d) reactions measured with the bubble chamber Gargamelle filled with light propane-freon mixture and exposed to the CERN-PS beams $[131,164]$. The fluxes are borrowed from Ref. [131,132]. The vertical error bars represent the total experimental errors including the $v_{\mu}$ or $\bar{v}_{\mu}$ flux normalization uncertainties

malization and energy spectra used in the GGM analysis. According to author's explanation, the uncertainties in the $v_{\mu}$ and $\bar{v}_{\mu}$ spectra are estimated to be $9 \%$ and $12 \%$ for the energy ranges between 2 and $6 \mathrm{GeV}$ and above $6 \mathrm{GeV}$, respectively. Below $2 \mathrm{GeV}$, the $v_{\mu} / \bar{v}_{\mu}$ spectra cannot be measured or adjusted in the GGM experiment and therefore an extrapolation from the higher energies has been used, which is not very reliable. Moreover, the $v_{\mu} / \bar{v}_{\mu}$ fluxes were estimated by using the observed number of elastic events and the expected (simulated) elastic cross section. The author's rough estimation of the number of inclusive events gives about 2500 and $1000 v_{\mu}$ and $\bar{v}_{\mu}$ events, respectively, but the number of elastic events separated from the full inclusive sample is uncertain.

Figure 36 shows the flux-weighted differential cross sections measured with the large heavy-liquid bubble chamber Gargamelle exposed to $v_{\mu}$ and $\bar{v}_{\mu}$ beams at CERN [129131,164]. The total error on the flux used in CERN GGM 


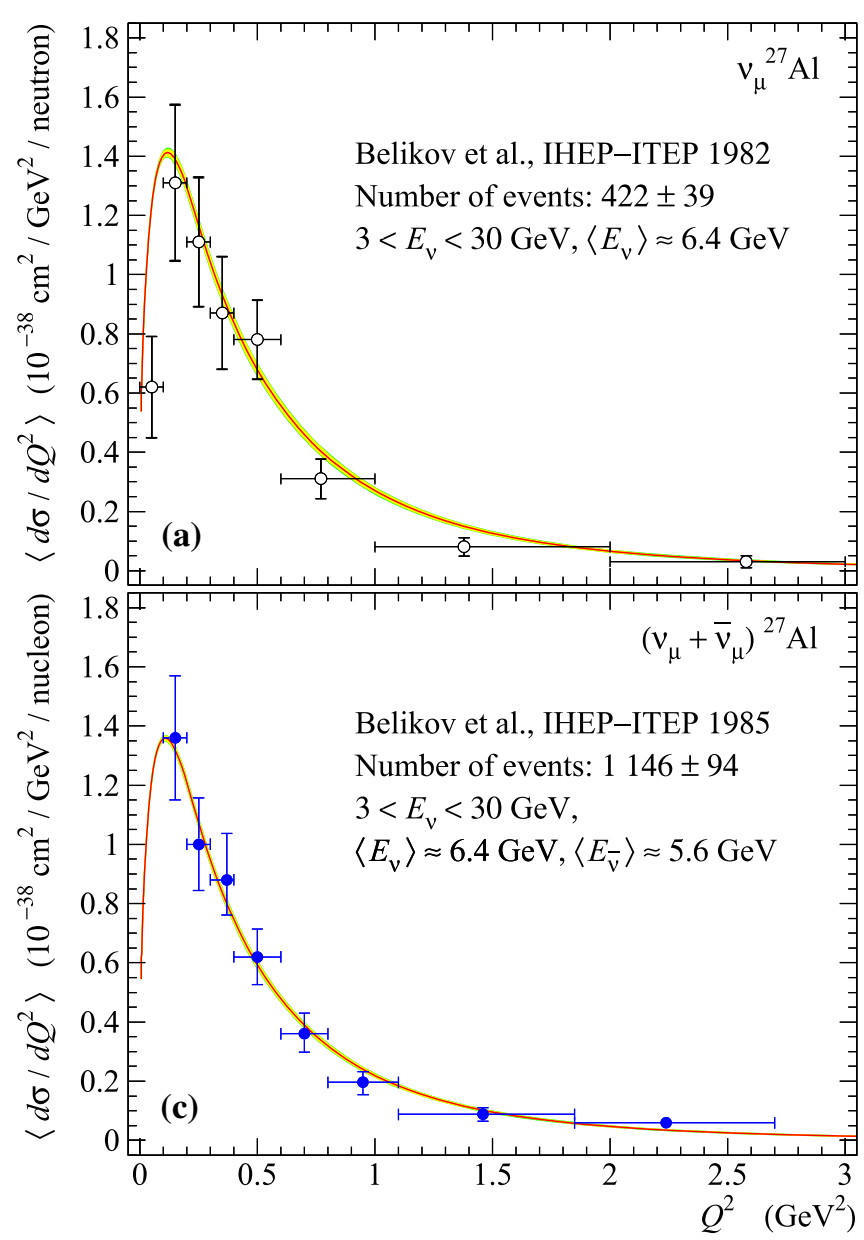

Fig. 37 Flux-weighted differential cross sections per interacting nucleon for $v_{\mu}$ (panel a) and $\bar{v}_{\mu}$ (panel b) CCQE reactions, and semisum of the $v_{\mu}$ and $\bar{v}_{\mu}$ cross sections (panel c) as measured in the IHEPITEP experiment using a spark chamber detector with aluminum filters exposed to the U70 broad-band $v_{\mu}$ and $\bar{v}_{\mu}$ beams of the Serpukhov PS $[136,137,139]$. The error bars in panels a-c include the uncertainties

1977 experiment ranges from $9 \%$ and to $\sim 12 \%$ in the energy regions dominated by neutrinos from pion and kaon decays, respectively. Systematic errors of $10 \%$ in the experimental data of CERN GGM 1979 [131] arise due to nuclear corrections (including FSI) and uncertainties in the shape and absolute normalization of the $v_{\mu}$ flux. The data of CERN GGM 1978 [164] are rescaled to the cross sections per neutron and proton of freon nucleus. The total errors include $12 \%$ uncertainty of the (anti)neutrino fluxes.

Panels (a)-(c) in Fig. 37 show the differential cross sections measured with the IHEP-ITEP experiment $[136,137$, 139]. The systematic errors in these measurement make up about $10 \%$ and arise mainly from the neutrino and antineutrino flux normalization (3\% - record-low uncertainty) and errors in scanning (3\%) and trigger (4\%) efficiencies [138]. In the analysis, we only use the data from Ref. [139], as they are based on much more extensive statistics. Panel (d) shows

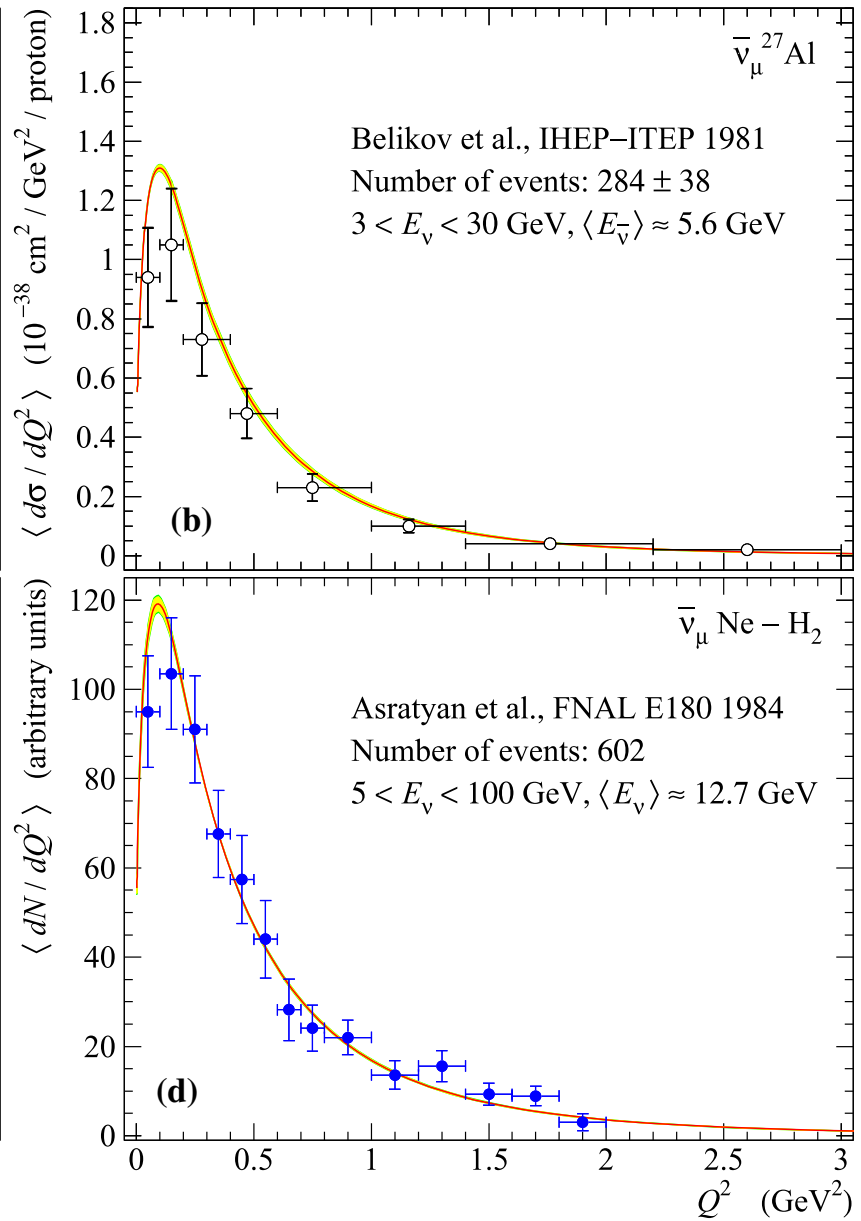

due to the flux normalization and nuclear Monte Carlo. Panel d shows the $Q^{2}$ distribution per proton for $\bar{v}_{\mu} \mathrm{CCQE}$ interactions, as measured in the FNAL E180 experiment with the 15-ft bubble chamber filled with heavy neon-hydrogen mixture $[107,108]$. The vertical error bars represent the total experimental errors including the $\bar{v}_{\mu}$ flux normalization uncertainties

the $Q^{2}$ distribution for $\bar{v}_{\mu}$ induced CCQE scattering measured in the FNAL E180 experiment with the 15-ft bubble chamber filled with heavy neon-hydrogen mixture (64\% of neon atoms) and exposed to the FNAL wide-band $\bar{v}_{\mu}$ beam $[107,108]$. Predicted distribution is calculated using the mean $\bar{v}_{\mu}$ energy of $12.7 \mathrm{GeV}$.

\section{B.2.1 Normalization factors for selected datasets}

The normalization factors and corresponding values of $\chi^{2} /$ ndf obtained from individual fits to each experimental dataset discussed in Sect. 3 are listed in Table 13. The data types used for fitting are also shown. 
Table 13 Results of individual fits of selected sets of experimental data (published before 2016) for the true CCQE (anti)neutrino-nucleus interactions. Shown are the data types, normalization factors, $\mathscr{N}$, with the estimated $1 \sigma$ and $2 \sigma$ uncertainties (the latters are in parentheses), and also the values of $\chi^{2} / \mathrm{ndf}$, where ndf $=\max (\mathrm{NP}-1,1)$ and NP is the number of the experimental data points (bins) in the corresponding dataset. The differential cross sections and $Q^{2}$ distributions are spec-

\begin{tabular}{|c|c|c|c|c|}
\hline Authors/Experiment/Date & Ref. & Data type & $\mathscr{N}$ & $\chi^{2} / \mathrm{ndf}$ \\
\hline \multicolumn{5}{|l|}{$\begin{array}{l}\text { Deuterium, hydrogen, and nuclear targets } \\
\text { recalculated to bare nucleons }\end{array}$} \\
\hline Mann et al., ANL 1972 & [94] & $\sigma_{v}$ & $0.79 \pm 0.07(0.14)$ & $5.92 / 5=1.18$ \\
\hline Mann et al., ANL 1973 & {$[95]$} & $\sigma_{v}$ & $0.94 \pm 0.07(0.14)$ & $4.13 / 6=0.69$ \\
\hline Barish et al., ANL 1975 & [146] & $\sigma_{v}$ & $0.99 \pm 0.05(0.09)$ & $7.86 / 7=1.12$ \\
\hline Singer et al., ANL 1977 & {$[98]$} & $\sigma_{v}$ & $0.78 \pm 0.05(0.09)$ & $8.43 / 7=1.20$ \\
\hline Barish et al., ANL 1977 & [97] & $\sigma_{v}^{*}$ & $0.89 \pm 0.03(0.07)$ & $16.0 / 7=2.28$ \\
\hline Miller et al., ANL 1982 & [99] & $d N_{\nu} / d Q^{2 *}$ & - & $73.9 / 38=1.95$ \\
\hline \multirow[t]{2}{*}{ Fanourakis et al., BNL 1980} & [79] & $d N_{\bar{\nu}} / d Q^{2 *}$ & - & $1.37 / 4=0.34$ \\
\hline & & $\sigma_{\bar{v}}$ & - & 0.05 \\
\hline Kitagaki et al., BNL 1990 (updated, 2003) & {$[104,226]$} & $d N_{\nu} / d Q^{2 *}$ & - & $21.5 / 36=0.60$ \\
\hline \multirow[t]{2}{*}{ Kitagaki et al., FNAL 1983} & {$[106]$} & $d N_{v} / d Q^{2 *}$ & - & $8.53 / 19=0.45$ \\
\hline & & $\sigma_{v}$ & $1.02 \pm 0.08(0.15)$ & $0.18 / 1=0.18$ \\
\hline \multirow[t]{2}{*}{ Allasia et al., CERN BEBC 1990} & [134] & $d \sigma_{\nu} / d Q^{2 *}$ & $0.94 \pm 0.04(0.07)$ & $4.77 / 7=0.68$ \\
\hline & & $\sigma_{v}$ & $0.87 \pm 0.03(0.06)$ & $15.1 / 5=3.02$ \\
\hline \multicolumn{5}{|l|}{ Heavy targets converted to bare nucleons } \\
\hline \multirow[t]{2}{*}{ Lyubushkin et al., CERN NOMAD 2009} & {$[34]$} & $\sigma_{v}$ & $1.05 \pm 0.03(0.05)$ & $7.49 / 9=0.83$ \\
\hline & & $\sigma_{\bar{v}}$ & $1.04 \pm 0.06(0.11)$ & $2.85 / 5=0.57$ \\
\hline \multirow[t]{4}{*}{ Brunner et al., IHEP SKAT 1990} & {$[142,152]$} & $d \sigma_{\nu} / d Q^{2 *}$ & $1.08 \pm 0.06(0.12)$ & $3.89 / 7=0.56$ \\
\hline & & $\sigma_{v}$ & $1.06 \pm 0.06(0.12)$ & $3.14 / 7=0.45$ \\
\hline & & $d \sigma_{\bar{v}} / d Q^{2 *}$ & $0.90 \pm 0.06(0.13)$ & $7.31 / 6=1.22$ \\
\hline & & $\sigma_{\bar{v}}$ & $0.82 \pm 0.06(0.11)$ & $8.10 / 6=1.35$ \\
\hline Makeev et al., IHEP SKAT 1981 & {$[80]$} & $\sigma_{v}$ & $0.74 \pm 0.06(0.11)$ & $6.03 / 3=2.01$ \\
\hline \multirow[t]{4}{*}{ Grabosch et al., IHEP SKAT 1988} & {$[141]$} & $d \sigma_{v} / d Q^{2}$ & $0.85 \pm 0.05(0.10)$ & $37.2 / 7=5.32$ \\
\hline & & $\sigma_{v}$ & $1.03 \pm 0.06(0.11)$ & $4.30 / 6=0.72$ \\
\hline & & $d \sigma_{\bar{v}} / d Q^{2}$ & $0.85 \pm 0.10(0.20)$ & $3.75 / 3=1.25$ \\
\hline & & $\sigma_{\bar{v}}$ & $0.75 \pm 0.09(0.18)$ & $5.40 / 3=1.80$ \\
\hline \multicolumn{5}{|l|}{ Heavy targets } \\
\hline \multirow[t]{2}{*}{ Kustom et al., ANL 1969} & {$[93]$} & $d \sigma_{v} / d Q^{2}$ & $0.60 \pm 0.03(0.06)$ & $185 / 13=14.21$ \\
\hline & & $\sigma_{v}$ & $1.12 \pm 0.05(0.10)$ & $12.4 / 5=2.47$ \\
\hline \multirow[t]{2}{*}{ Ahrens et al., BNL 1985} & [102] & $d \sigma_{v_{e}} / d Q^{2}$ & $0.92 \pm 0.08(0.15)$ & $14.6 / 9=1.62$ \\
\hline & & $d \sigma_{\nu} / d Q^{2}$ & $0.64 \pm 0.03(0.06)$ & $15.1 / 8=1.89$ \\
\hline \multirow[t]{2}{*}{ Asratyan et al., FNAL 1984} & {$[107]$} & $d N_{\bar{\nu}} / d Q^{2 *}$ & - & $7.93 / 13=0.61$ \\
\hline & & $\sigma_{\bar{v}}$ & - & 0.06 \\
\hline \multirow[t]{2}{*}{ Suwonjandee et al., FNAL NuTeV 2004} & [110] & $\sigma_{v}$ & $1.10 \pm 0.04(0.08)$ & $21.0 / 16=1.31$ \\
\hline & & $\sigma_{\bar{v}}$ & $1.29 \pm 0.04(0.08)$ & $21.7 / 16=1.36$ \\
\hline Alcaraz-Aunion et al., FNAL SciBooNE 2010 & {$[15]$} & $\sigma_{v}$ & $0.95 \pm 0.03(0.06)$ & $1.89 / 4=0.47$ \\
\hline $\begin{array}{l}\text { Aguilar-Arevalo et al., FNAL MiniBooNE }\left(\mathrm{CH}_{2}\right) \\
2010\end{array}$ & {$[13]$} & $d^{2} \sigma_{\nu} / d E_{\mu} d \cos \theta_{\mu}^{*}$ & $1.00 \pm 0.01(0.02)$ & $35.8 / 136=0.26$ \\
\hline
\end{tabular}

trum averaged. The cross sections for hydrogen and deuterium targets as well as those obtained with other targets, but converted by the authors into the data on bare nucleons, are calculated with the current axial mass, $M_{A}=M_{0}$; in all other cases (marked with an asterisk), we use the running axial mass, $M_{A}^{\text {run }}$, with the parameters $M_{0}$ and $E_{0}$ obtained in the global fit (see Eq. (7)) 
Table 13 continued

\begin{tabular}{|c|c|c|c|c|}
\hline Authors/Experiment/Date & Ref. & Data type & $\mathscr{N}$ & $\chi^{2} / \mathrm{ndf}$ \\
\hline $\begin{array}{l}\text { Aguilar-Arevalo et al., FNAL MiniBooNE }\left(\mathrm{CH}_{2}\right) \\
2013\end{array}$ & {$[14]$} & $d^{2} \sigma_{\bar{v}} / d E_{\mu} d \cos \theta_{\mu}^{*}$ & $1.01 \pm 0.01(0.03)$ & $31.9 / 77=0.42$ \\
\hline FNAL MiniBooNE $\left({ }^{12}\right.$ C) 2013 & {$[14]$} & $d^{2} \sigma_{\bar{v}} / d E_{\mu} d \cos \theta_{\mu}$ & $1.01 \pm 0.01(0.03)$ & $44.7 / 74=0.61$ \\
\hline $\begin{array}{l}\text { Fiorentini et al., FNAL MINERvA } 2016 \text { ( } v \text {, } \\
\left.\theta_{\mu} \leq 20^{\circ}\right)\end{array}$ & {$[17]$} & $d \sigma_{\nu} / d Q^{2}$ & - & $11.24 / 8=1.41$ \\
\hline FNAL MINERvA 2016 ( $v$, no cut) & & & - & $12.15 / 8=1.52$ \\
\hline $\begin{array}{l}\text { Fields et al., FNAL MINER } v \text { A } 2016(\bar{v} \text {, } \\
\left.\theta_{\mu} \leq 20^{\circ}\right)\end{array}$ & [18] & $d \sigma_{\bar{v}} / d Q^{2}$ & - & $5.06 / 8=0.63$ \\
\hline FNAL MINERvA 2016 ( $\bar{v}$, no cut) & & & - & $5.62 / 8=0.70$ \\
\hline FNAL MINER $v$ A $2016\left(v+\bar{v}, \theta_{\mu} \leq 20^{\circ}\right)$ & {$[17,18]$} & $d \sigma_{v} / d Q^{2} \& d \sigma_{\bar{v}} / d Q^{2}$ & - & $19.66 / 16=1.23$ \\
\hline FNAL MINER $v$ A $2016(v+\bar{v}$, no cut $)$ & & & - & $22.08 / 16=1.38$ \\
\hline \multirow[t]{2}{*}{ Franzinetti,CERN HLBC 1966} & {$[122]$} & $\sigma_{v}$ & $0.80 \pm 0.14(0.27)$ & $10.8 / 4=2.70$ \\
\hline & & $\sigma_{\bar{v}}$ & $1.07 \pm 0.29(0.57)$ & $0.89 / 1=0.89$ \\
\hline \multirow[t]{2}{*}{ Young et al., CERN HLBC 1967} & {$[123]$} & $\sigma_{v}$ & $0.64 \pm 0.07(0.15)$ & $4.99 / 4=1.25$ \\
\hline & & $\sigma_{\bar{v}}$ & $0.87 \pm 0.18(0.36)$ & $1.51 / 1=1.51$ \\
\hline Budagov et al., CERN HLBC 1969 & {$[126]$} & $\sigma_{v}$ & $0.51 \pm 0.05(0.10)$ & $18.2 / 4=4.55$ \\
\hline \multirow[t]{2}{*}{ Eichten et al., CERN GGM 1973} & {$[76]$} & $\sigma_{\nu}$ & $0.74 \pm 0.04(0.08)$ & $9.66 / 12=0.81$ \\
\hline & & $\sigma_{\bar{v}}$ & $0.83 \pm 0.05(0.10)$ & $8.47 / 11=0.77$ \\
\hline \multirow[t]{2}{*}{ Rollier et al., CERN GGM 1975} & {$[128,146]$} & $\sigma_{\nu}$ & $0.86 \pm 0.04(0.08)$ & $6.44 / 8=0.81$ \\
\hline & & $\sigma_{\bar{v}}$ & $0.85 \pm 0.05(0.10)$ & $8.33 / 11=0.76$ \\
\hline \multirow[t]{4}{*}{ Bonetti et al., CERN GGM 1977} & {$[129]$} & $d \sigma_{\nu} / d Q^{2}$ & $0.79 \pm 0.03(0.07)$ & $18.8 / 11=1.71$ \\
\hline & & $\sigma_{v}$ & $0.80 \pm 0.04(0.08)$ & $10.8 / 12=0.90$ \\
\hline & & $d \sigma_{\bar{v}} / d Q^{2}$ & $0.82 \pm 0.04(0.08)$ & $8.68 / 7=1.24$ \\
\hline & & $\sigma_{\bar{v}}$ & $0.84 \pm 0.05(0.09)$ & $12.6 / 9=1.40$ \\
\hline \multirow[t]{2}{*}{ Rollier et al., CERN GGM 1978} & [164] & $d \sigma_{\bar{v}} / d Q^{2}$ & $0.88 \pm 0.03(0.06)$ & $29.3 / 11=2.67$ \\
\hline & & $\sigma_{\bar{v}}$ & $0.85 \pm 0.03(0.06)$ & $23.2 / 10=2.32$ \\
\hline \multirow[t]{2}{*}{ Pohl et al., CERN GGM 1979} & {$[131]$} & $d \sigma_{\nu} / d Q^{2}$ & $0.79 \pm 0.04(0.08)$ & $13.1 / 6=2.19$ \\
\hline & & $\sigma_{\nu}$ & $0.77 \pm 0.04(0.08)$ & $11.9 / 6=1.98$ \\
\hline Armenise et al., CERN GGM 1979 & {$[132,133]$} & $\sigma_{\bar{v}}$ & $0.78 \pm 0.03(0.06)$ & $20.6 / 10=2.06$ \\
\hline De la Ossa Romero, CERN LAr-TPC 2007 & [135] & $\sigma_{v}^{*}$ & - & 0.09 \\
\hline \multirow[t]{2}{*}{ Lyubushkin et al., CERN NOMAD 2009} & {$[34]$} & $\sigma_{\nu}^{*}$ & $1.04 \pm 0.03(0.05)$ & $5.91 / 9=0.66$ \\
\hline & & $\sigma_{\bar{\nu}}^{*}$ & $1.02 \pm 0.06(0.11)$ & $3.09 / 5=0.62$ \\
\hline \multirow[t]{3}{*}{ Belikov et al., IHEP-ITEP 1981} & [136] & $\sigma_{v}$ & $0.90 \pm 0.06(0.12)$ & $1.97 / 5=0.40$ \\
\hline & & $d \sigma_{\bar{v}} / d Q^{2}$ & $0.91 \pm 0.05(0.11)$ & $2.07 / 7=0.30$ \\
\hline & & $\sigma_{\bar{v}}$ & $0.90 \pm 0.06(0.11)$ & $3.21 / 5=0.80$ \\
\hline \multirow[t]{2}{*}{ Belikov et al., IHEP-ITEP 1982} & [137] & $d \sigma_{v} / d Q^{2}$ & $0.88 \pm 0.06(0.11)$ & $10.2 / 7=1.46$ \\
\hline & & $\sigma_{v}$ & $0.90 \pm 0.06(0.12)$ & $1.78 / 5=0.36$ \\
\hline \multirow[t]{4}{*}{ Belikov et al., IHEP-ITEP 1985} & {$[138,139]$} & $d \sigma_{v+\bar{v}} / d Q^{2 *}\left\{\begin{array}{l}v: \\
\bar{v}:\end{array}\right.$ & $0.98 \pm 0.11(0.18)$ & $5.68 / 6=0.95$ \\
\hline & & & $0.99 \pm 0.13(0.21)$ & \\
\hline & & $\sigma_{\nu}^{*}$ & $0.87 \pm 0.04(0.07)$ & $7.02 / 7=1.00$ \\
\hline & & $\sigma_{\bar{\nu}}^{*}$ & $0.90 \pm 0.05(0.09)$ & $2.66 / 7=0.38$ \\
\hline Abe et al., T2K ND280 (off-axis) 2014 & {$[23]$} & $\sigma_{v}$ & - & $9.57 / 5=1.91$ \\
\hline Abe et al., T2K INGRID (on-axis) 2015 & {$[26]$} & $\sigma_{\nu}^{*}$ & $0.98 \pm 0.09(0.17)$ & $0.55 / 1=0.55$ \\
\hline
\end{tabular}




\section{References}

1. O. Benhar, P. Huber, C. Mariani, D. Meloni, Phys. Rep. 700, 1 (2017). arXiv:1501.06448 [nucl-th]

2. U. Mosel, J. Phys. G 46(11), 113001 (2019). arXiv:1904.11506 [hep-ex]

3. A. Bodek, S.E. Avvakumov, R.K. Bradford Jr., H.S. Budd, Modeling atmospheric neutrino interactions: duality constrained parameterization of vector and axial nucleon form factors (2007). arXiv:0708.1827 [hep-ex]

4. A. Bodek, S.E. Avvakumov, R.K. Bradford Jr., H.S. Budd, Eur. Phys. J. C 53, 349 (2008). arXiv:0708.1946 [hep-ex]

5. A. Bodek, S.E. Avvakumov, R.K. Bradford Jr., H.S. Budd, J. Phys. Conf. Ser. 110, 082004 (2008). arXiv:0709.3538 [hep-ex]

6. K.S. Kuzmin, V.V. Lyubushkin, V.A. Naumov, Phys. Atom. Nucl. 69(11), 1857 (2006). arXiv:hep-ph/0511308

7. K.S. Kuzmin, V.V. Lyubushkin, V.A. Naumov, Acta Phys. Pol. B 37, 2337 (2006). arXiv:hep-ph/0606184

8. K.S. Kuzmin, V.V. Lyubushkin, V.A. Naumov, Eur. Phys. J. C 54, 517 (2008). arXiv:0712.4384 [hep-ph]

9. S.K. Singh, Nucl. Phys. B 36(2), 419 (1972)

10. S.K. Singh, Phys. Rev. D 10(3), 988 (1974)

11. S.K. Singh, H. Arenhovel, Z. Phys. A 324, 347 (1986)

12. R.A. Smith, E.J. Moniz, Nucl. Phys. B 43, 605 (1972) [Erratum: ibid. 101(2), 547 (1975)]

13. A.A. Aguilar-Arevalo et al. (MiniBooNE Collaboration), Phys. Rev. D 81(9), 092005 (2010). arXiv:1002.2680 [hep-ex]

14. A.A. Aguilar-Arevalo et al. (MiniBooNE Collaboration), Phys. Rev. D 88(3), 032001 (2013). arXiv:1301.7067 [hep-ex]

15. J.L. Alcaraz-Aunion, J. Walding (SciBooNE Collaboration), AIP Conf. Proc. 1189, 145 (2009). arXiv:0909.5647 [hep-ex]

16. J.L. Alcaraz Aunion, Ph.D. thesis, Barcelona, IFAE (2010). http:// lss.fnal.gov/cgi-bin/find_paper.pl?thesis-2010-45

17. G.A. Fiorentini et al. (MINERvA Collaboration), Phys. Rev. Lett. 111(2), 022502 (2013). arXiv:1305.2243 [hep-ex]

18. L. Fields et al. (MINERvA Collaboration), Phys. Rev. Lett. 111(2), 022501 (2013). arXiv:1305.2234 [hep-ex]

19. T. Walton et al. (MINERvA Collaboration), Phys. Rev. D 91(7), 071301 (2015). arXiv:1409.4497 [hep-ex]

20. P. Adamson et al. (MINOS Collaboration), Phys. Rev. D 91(1), 012005 (2015). arXiv:1410.8613 [hep-ex]

21. K. Abe et al. (T2K Collaboration), Phys. Rev. D 87(9), 092003 (2013). arXiv:1302.4908 [hep-ex]

22. D. Hadley (for the T2K Collaboration), PoS EPS-HEP 2013, 008 (2013)

23. K. Abe et al. (T2K Collaboration), Phys. Rev. D 92(11), 112003 (2015). arXiv:1411.6264 [hep-ex]

24. K. Abe et al. (T2K Collaboration), Phys. Rev. D 93(11), 112012 (2016). arXiv:1602.03652 [hep-ex]

25. K. Abe et al. (T2K Collaboration), Phys. Rev. D 98(3), 032003 (2018). arXiv:1802.05078 [hep-ex]

26. K. Abe et al. (T2K Collaboration), Phys. Rev. D 91(11), 112002 (2015). arXiv:1503.07452 [hep-ex]

27. J.M. Nieves, I. Ruiz-Simo, M.J. Vicente Vacas, J. Phys. Conf. Ser. 408, 012040 (2013). arXiv:1110.1200 [hep-ph]

28. J.M. Nieves, I. Ruiz Simo, M.J. Vicente Vacas, Phys. Lett. B 707(1), 72 (2012). arXiv:1106.5374 [hep-ph]

29. C. Juszczak, J.T. Sobczyk, J. Zmuda, Phys. Rev. C 82(4), 045502 (2010). arXiv:1007.2195 [nucl-th]

30. T. Golan, K.M. Graczyk, C. Juszczak, J.T. Sobczyk, Phys. Rev. C 88(2), 024612 (2013). arXiv:1302.3890 [hep-ph]

31. A.V. Butkevich, D. Perevalov, Phys. Rev. D 89(5), 053014 (2014). arXiv:1311.3754 [hep-ph]

32. C. Wilkinson et al., Phys. Rev. D 93(7), 072010 (2016). arXiv:1601.05592 [hep-ex]
33. V. Bernard, L. Elouadrhiri, U.G. Meißner, J. Phys. G 28(1), R1 (2002). arXiv:hep-ph/0107088

34. V.V. Lyubushkin et al. (NOMAD Collaboration), Eur. Phys. J. C 63, 355 (2009). arXiv:0812.4543 [hep-ex]

35. R.W. Gran et al. (K2K Collaboration), Phys. Rev. D 74(5), 052002 (2006). arXiv:hep-ex/0603034

36. X. Espinal, F.N. Sánchez, AIP Conf. Proc. 967, 117 (2007)

37. M. Sajjad Athar, F. Akbar, M. Rafi Alam, S. Chauhan, S.K. Singh, F. Zaidi, Lepton production cross sections in quasielastic $v / \bar{v}-\mathrm{a}$ scattering (2016). arXiv:1611.07166 [nucl-th]

38. K.M. Graczyk, Local density and the RPA corrections in charge current quasielastic neutrino on oxygen, Argon and Iron scattering (2004). arXiv:nucl-th/0401053

39. O. Benhar, N. Farina, H. Nakamura, M. Sakuda, R. Seki, Phys. Rev. D 72(5), 053005 (2005). arXiv:hep-ph/0506116

40. O. Benhar, D. Meloni, Nucl. Phys. A 789(1-4), 379 (2007). arXiv:hep-ph/0610403

41. A.M. Ankowski, O. Benhar, M. Sakuda, Phys. Rev. D 91(3), 033005 (2015). arXiv:1404.5687 [nucl-th]

42. E. Vagnoni, O. Benhar, D. Meloni, Phys. Rev. Lett. 118(14), 142502 (2017). arXiv:1701.01718 [nucl-th]

43. N. Rocco, C. Barbieri, O. Benhar, A. De Pace, A. Lovato, Phys. Rev. C 99(2), 025502 (2019). arXiv:1810.07647 [nucl-th]

44. M.V. Ivanov, A.N. Antonov, J.A. Caballero, G.D. Megias, M.B. Barbaro, E. Moya de Guerra, J.M. Udias, Phys. Rev. C 89(1), 014607 (2014). arXiv:1312.5357 [nucl-th]

45. A. Bodek, M.E. Christy, B. Coopersmith, Eur. Phys. J. C 74(10), 3091 (2014). arXiv:1405.0583 [hep-ph]

46. J.E. Sobczyk, Phys. Rev. C 96(4), 045501 (2017). arXiv:1706.06739 [nucl-th]

47. M.V. Ivanov, A.N. Antonov, G.D. Megias, J.A. Caballero, M.B. Barbaro, J.E. Amaro, I Ruiz Simo, T.W. Donnelly, J.M. Udias, Phys. Rev. C 99(1), 014610 (2019). arXiv:1812.09435 [nucl-th]

48. M.V. Ivanov, A.N. Antonov, G.D. Megias, J.A. Caballero, M.B. Barbaro, J.E. Amaro, I Ruiz Simo, T.W. Donnelly, J.M. Udias, AIP Conf. Proc. 2075, 070004 (2019)

49. M.B. Barbaro, J.E. Amaro, J.A. Caballero, T.W. Donnelly, J.M. Udias, AIP Conf. Proc. 1441, 417 (2012). arXiv:1108.5202 [nuclth]

50. A. Meucci, C. Giusti, Phys. Rev. D 89(11), 117301 (2014). arXiv:1404.3554 [nucl-th]

51. M. Ericson, M. Martini, Phys. Rev. C 91(3), 035501 (2015). arXiv:1501.02442 [nucl-th]

52. R.W. Gran, J.M. Nieves, F.N. Sánchez, MJ Vicente. Vacas, Phys. Rev. D 88(11), 113007 (2013). arXiv:1307.8105 [hep-ph]

53. T. Van Cuyck, N. Jachowicz, R. González-Jiménez, M. Martini, V. Pandey, J. Ryckebusch, N. Van Dessel, Phys. Rev. C 94(2), 024611 (2016). arXiv:1606.00273 [nucl-th]

54. J.M. Nieves, I. Ruiz Simo, M.J. Vicente Vacas, Phys. Lett. B 721(1-3), 90 (2013). arXiv:1302.0703 [hep-ph]

55. V. Pandey, N. Jachowicz, J. Ryckebusch, T. Van Cuyck, W. Cosyn, Phys. Rev. C 89(2), 024601 (2014). arXiv:1310.6885 [nucl-th]

56. O.D. Lalakulich, U. Mosel, K. Gallmeister, Phys. Rev. C 86(5), 054606 (2012). arXiv:1208.3678 [nucl-th]

57. U. Mosel, K. Gallmeister, Phys. Rev. C 97(4), 045501 (2018). arXiv: 1712.07134 [hep-ex]

58. A. Bodek, H.S. Budd, M.E. Christy, Eur. Phys. J. C 71, 1726 (2011). arXiv:1106.0340 [hep-ph]

59. J.T. Sobczyk, Eur. Phys. J. C 72, 1850 (2012). arXiv:1109.1081 [hep-ex]

60. A. Bodek, U. Sarica, K.S. Kuzmin, V.A. Naumov, AIP Conf. Proc. 1560, 193 (2013). arXiv:1207.1247 [hep-ex]

61. G.D. Megias, M.V. Ivanov, R. González-Jiménez, M.B. Barbaro, J.A. Caballero, T.W. Donnelly, J.M. Udías, Phys. Rev. D 89(9), 093002 (2014). arXiv:1402.1611 [nucl-th] [Erratum: ibid. 91(3), 039903(E) (2015)] 
62. M.B. Barbaro, A. De Pace, L. Fiume, Universe 7(5), 140 (2021). arXiv:2104.10472 [hep-ph]

63. R. González-Jiménez, G.D. Megias, M.B. Barbaro, J.A. Caballero, T.W. Donnelly, Phys. Rev. C 90(3), 035501 (2014). arXiv: 1407.8346 [nucl-th]

64. G.D. Megias, M.B. Barbaro, J.A. Caballero, J.E. Amaro, T.W. Donnelly, I. Ruiz Simo, J.W. Van Orden, J. Phys. G 46(1), 015104 (2019). arXiv:1711.00771 [nucl-th]

65. G.D. Megias, J.E. Amaro, M.B. Barbaro, J.A. Caballero, T.W. Donnelly, I. Ruiz Simo, Phys. Rev. D 94(9), 093004 (2016). arXiv:1607.08565 [nucl-th]

66. G.D. Megias, M.B. Barbaro, J.A. Caballero, Phys. Rev. D 99(11), 113002 (2019). arXiv:1807.10532 [nucl-th]

67. J.E. Amaro, M.B. Barbaro, J.A. Caballero, R. González-Jiménez, G.D. Megias, I. Ruiz Simo, J. Phys. G 47(12), 124001 (2020). arXiv:1912.10612 [nucl-th]

68. I. Ruiz Simo, V.L. Martinez-Consentino, J.E. Amaro, E. Ruiz Arriola, Phys. Rev. D 97(11), 116006 (2018). arXiv:1804.07548 [nucl-th]

69. S. Boyd, S. Dytman, E. Hernandez, J.T. Sobczyk, R. Tacik, AIP Conf. Proc. 1189, 60 (2009)

70. H. Gallagher, G. Garvey, G.P. Zeller, Annu. Rev. Nucl. Part. Sci. 61, 355 (2011)

71. G.T. Garvey, D.A. Harris, H.A. Tanaka, R.L. Tayloe, G.P. Zeller, Phys. Rep. 580, 1 (2015). arXiv:1412.4294 [hep-ex]

72. L. Alvarez-Ruso, Y. Hayato, J.M. Nieves, New J. Phys. 16, 075015 (2014). arXiv:1403.2673 [hep-ph]

73. T. Katori, M. Martini, J. Phys. G 45(1), 013001 (2018). arXiv:1611.07770 [hep-ph]

74. M. Betancourt et al., Phys. Rep. 773-774, 1 (2018). arXiv:1805.07378 [hep-ex]

75. O. Erriquez et al., Phys. Lett. 80 B(3), 309 (1979)

76. T. Eichten et al., Phys. Lett. B 46(2), 274 (1973)

77. S. Barish et al., Phys. Lett. 66 B(3), 291 (1977)

78. A.E. Asratyan et al., Phys. Lett. 76 B(2), 239 (1978)

79. G.K. Fanourakis, L.K. Resvanis, G.A. Grammatikakis, P. Tsilimigras, A. Vayaki, U. Camerini, W.F. Fry, R.J. Loveless, J.H. Mapp, D.D. Reeder, Phys. Rev. D 21(3), 562 (1980)

80. V.V. Makeev et al., JETP Lett. 34(7), 397 (1981)

81. E.J. Moniz, I. Sick, R.R. Whitney, J.R. Ficenec, R.D. Kephart, W.P. Trower, Phys. Rev. Lett. 26(8), 445 (1971)

82. R.R. Whitney, I. Sick, J.R. Ficenec, R.D. Kephart, W.P. Trower, Phys. Rev. C 9(6), 2230 (1974)

83. P.D. Zimmerman, M.R. Yearian, Z. Phys. A 278(3), 291 (1976)

84. A. Bodek, T. Cai, Eur. Phys. J. C 79(4), 293 (2019). arXiv:1801.07975 [nucl-th]

85. T. Cai et al. (MINERvA Collaboration), Phys. Rev. D 101(9), 092001 (2020). arXiv:1910.08658 [hep-ex]

86. A. Bodek, J.L. Ritchie, Phys. Rev. D 23(5), 1070 (1981)

87. L. Alvarez-Ruso et al. (GENIE Collaboration), Eur. Phys. J. Spec. Top. (2021). https://doi.org/10.1140/epjs/s11734-021-00295-7. arXiv:2106.09381 [hep-ph]

88. K.S. Kuzmin, V.V. Lyubushkin, V.A. Naumov, Mod. Phys. Lett. A 19(38), 2815 (2004). arXiv:hep-ph/0312107

89. C. Berger, L.M. Sehgal, Phys. Rev. D 76(11), 113004 (2007). arXiv:0709.4378 [hep-ph] [Erratum: ibid. 77(5), 059901(E) (2008)]

90. D. Rein, L.M. Sehgal, Ann. Phys. 133(1), 79 (1981)

91. I.D. Kakorin, K.S. Kuzmin, Phys. Rev. D 104(9), 093001 (2021) arXiv:2109.14681 [hep-ph]

92. P.A. Zyla et al. (Particle Data Group), Prog. Theor. Exp. Phys. 2020(8), 083C01 (2020)

93. R.L. Kustom, D.E. Lundquist, T.B. Novey, A. Yokosawa, F. Chilton, Phys. Rev. Lett. 22(19), 1014 (1969)

94. W.A. Mann et al., Reported at 'ICHEP 72' (paper \#784); the data are published in Ref. [145] (1972)
95. W.A. Mann et al., Phys. Rev. Lett. 31(13), 844 (1973)

96. S.J. Barish et al., Technical Report. COO-1428-428, ANL-HEPCP-75-38, Argonne National Laboratory (1976)

97. S.J. Barish et al., Phys. Rev. D 16(11), 3103 (1977)

98. R.A. Singer, Study of the reaction $v+n \rightarrow \mu^{-}+p$, in Proceedings of the International Conference on Neutrino Physics and Astrophysics (Neutrino'1977), Baksan Valley, USSR, June 18-24, 1977, vol. 2, ed. by M.A. Markov, G.V. Domogatsky, A.A. Komar, A.N. Tavkhelidze ("Nauka", Moscow, 1978), pp. 95-104

99. K.L. Miller et al., Phys. Rev. D 26(3), 537 (1982)

100. A.M. Cnops et al., Neutrino-deuterium reactions in the 7 -ft bubble chamber, in Proceedings of the Topical Conference on Neutrino Physics at Accelerators, Oxford, England, UK, July 4-7, 1978, ed. by A.G. Michette, P.B. Renton (Science Research Council, Rutherford Laboratory, Chilton, 1978), pp. 62-67

101. N.J. Baker et al., Phys. Rev. D 23(11), 2499 (1981)

102. L.A. Ahrens et al., Phys. Rev. D 31(11), 2732 (1985)

103. L.A. Ahrens et al., Phys. Lett. B 202(2), 284 (1988)

104. T. Kitagaki et al., Phys. Rev. D 42(5), 1331 (1990)

105. K. Furuno et al., KEK Preprint 2003-48, RCNS-03-01. Reported at the 2nd International Workshop on Neutrino-Nucleus Interactions in the Few GeV Region (NuInt 2002), Irvine, California, USA, December 12-15, 2002 (2003). The paper presents a reanalysis of the data from Ref. [104]

106. T. Kitagaki et al., Phys. Rev. D 28(3), 436 (1983)

107. A.É. Asratyan et al., Sov. J. Nucl. Phys. 39(3), 392 (1984)

108. A.E. Asratyan et al., Phys. Lett. B 137(1-2), 122 (1984)

109. V.V. Ammosov et al., Z. Phys. C 36, 377 (1987)

110. N. Suwonjandee, Ph.D. thesis, Cincinnati University (2004). https://s3.cern.ch/inspire-prod-files-7/ 7584b3e90b28e6ae661e35f053425107

111. J. Wolcott et al. (MINERvA Collaboration), Phys. Rev. Lett. 116(8), 081802 (2016). arXiv: 1509.05729 [hep-ex]

112. X.G. Lu et al., Phys. Rev. C 94(1), 015503 (2016). arXiv:1512.05748 [hep-ex]

113. M. Betancourt et al. (MINERvA Collaboration), Phys. Rev. Lett. 119(8), 082001 (2017). arXiv:1705.03791 [hep-ex]

114. X.G. Lu et al. (MINERvA Collaboration), Phys. Rev. Lett. 121(2), 022504 (2018). arXiv:1805.05486 [hep-ex]

115. D. Ruterbories et al. (MINERvA Collaboration), Phys. Rev. D 99(1), 012004 (2019). arXiv:1811.02774 [hep-ex]

116. C.E. Patrick et al. (MINERvA Collaboration), Phys. Rev. D 97(5), 052002 (2018). arXiv:1801.01197 [hep-ex]

117. M. Betancourt, Ph.D. thesis, Minnesota University (2013). http:// 1ss.fnal.gov/archive/thesis/2000/fermilab-thesis-2013-10.pdf

118. E. Arrieta Díaz, Ph.D. thesis, Michigan State University (2014). http://lss.fnal.gov/archive/thesis/2000/fermilab-thesis-2014-24. shtml

119. L.B. Auerbach et al. (LSND Collaboration), Phys. Rev. C 66(1), 015501 (2002). arXiv:nucl-ex/0203011

120. M.M. Block et al., Phys. Lett. 12(3), 281 (1964). http://www. sciencedirect.com/science/article/pii/0031916364911047

121. H. Burmeister et al., Further analysis of the neutrino interactions in the CERN heave liquid bubble chamber, in Proceedings of the Experimental Neutrino Physics, CERN, Geneva, Switzerland, January 20-22, 1965, ed. by C. Franzinetti (CERN, Geneva, 1965), pp. 25-30. https://cds.cern.ch/record/276355

122. C. Franzinetti, Neutrino Interactions in the CERN Heavy Liquid Bubble Chamber, CERN Yellow Report No. 66-13. Lecture at the Meeting of the American Physical Society, Chicago, October 28, 1965 (CERN, Geneva, 1965). https://cds.cern.ch/record/276222

123. E.C.M. Young, Technical report, CERN. CERN Yellow Report No. 67-12 (1967). http://cds.cern.ch/record/276222

124. A. Orkin-Lecourtois, C.A. Piketty, Nuovo Cim. A 50(4), 927 (1967)

125. M. Holder et al., Nuovo Cim. A 57(2), 338 (1968) 
126. I. Budagov et al., Lett. Nuovo Cim. 2(15), 689 (1969)

127. M. Haguenauer (for the Aachen-Brussels-CERN-Ecole PolyOrsay-London Collaboration), Gargamelle experiment, in Proceedings of the 17th International Conference on High Energy Physics (ICHEP 1974), London, England, UK, July 1-10, 1974, ed. by J.R. Smith (Rutherford High Energy Laboratory, Didcot, Berkshire, 1975), pp. IV-95-100. http://inspirehep.net/record/ 1302859/files/c74-07-01-backmatter.pdf

128. M. Rollier (Gargamelle Neutrino Collaboration), Elastic neutrino and antineutrino interactions, in Proceedings of the International Colloquium on High Energy Neutrino Physics, Paris, France, March 18-20, 1975 (Éditions du Centre National de la Recherche Scientifique, Paris, 1975), pp. 349-355

129. S. Bonetti, G. Carnesecchi, D. Cavalli, P. Negri, A. Pullia, M. Rollier, F. Romano, R. Schira, Nuovo Cim. A 38(3), 260 (1977)

130. P. Musset, J.P. Vialle, Phys. Rep. 39, 1 (1978)

131. M. Pohl et al. (Gargamelle Neutrino Propane Collaboration), Lett. Nuovo Cim. 26(11), 332 (1979)

132. N. Armenise et al., Nucl. Phys. B 152(3-4), 365 (1979)

133. S.K. Singh, E. Oset, Nucl. Phys. A 542(4), 587 (1992)

134. D. Allasia et al. (Amsterdam-Bergen-Bologna-Padua-PisaSaclay-Torino Collaboration), Nucl. Phys. B 343(2), 285 (1990)

135. A.M. de la Ossa Romero, Ph.D. thesis, Granada University \& CAFPE, Granada (2007). http://inspirehep.net/record/746636/ files/thesis-2007-023.pdf. arXiv:hep-ex/0703026

136. S.V. Belikov et al. (IHEP-ITEP Collaboration), Technical Report. IFVE-81-146, Institute for High Energy Physics, Serpukhov (1981)

137. S.V. Belikov et al., Sov. J. Nucl. Phys. 35(1), 35 (1982)

138. S.V. Belikov et al., Z. Phys. A 320, 625 (1985)

139. S.V. Belikov, A.A. Volkov, V.I. Kochetkov, A.I. Mukhin, Y.M. Sviridov, K.E. Shestermanov, Sov. J. Nucl. Phys. 41(4), 589 (1985)

140. H.J. Grabosch et al. (SKAT Collaboration), Technical Report. PHE-86-11, Institute of High-Energy Physics, Zeuthen (1986)

141. H.J. Grabosch et al., Sov. J. Nucl. Phys. 47(6), 1630 (1988)

142. J. Brunner et al. (SKAT Collaboration), Z. Phys. C 45, 551 (1990)

143. K. Abe et al. (T2K), Phys. Rev. D 102(1), 012007 (2020). arXiv:1908.10249 [hep-ex]

144. K. Abe et al. (T2K Collaboration), Phys. Rev. D 97(1), 012001 (2018). arXiv:1708.06771 [hep-ex]

145. D.H. Perkins, Neutrino interactions, in Proceedings of the 16th International Conference on High-Energy Physics (ICHEP 1972), Batavia, Illinois, USA, September 6-13, 1972, vol. 4, ed. by J.D. Jackson, A. Roberts (National Accelerator Laboratory Publications Office, Batavia, 1973), pp. 189-247. http://inspirehep.net/ record/76469/files/v4p189.pdf

146. D.H. Perkins, Review of neutrino experiments, in Proceedings of the International Symposium on Lepton and Photon Interactions at High Energies, Leland Stanford Junior University, Stanford, California, USA, August 21-27, 1975, ed. by T.W. Kirk (SLAC, Stanford, 1976), pp. 571-603. http://inspirehep.net/ record/105037/files/Perkins_LP1975.pdf

147. M. Derrick, Charged current neutrino reactions in the resonance region, in Proceedings of the 17th International Conference on High-Energy Physics (ICHEP 1974), London, England, July 110, 1974, ed. by J.R. Smith (Science Research Council, Rutherford Laboratory, Chilton, Didcot, 1975), pp. II.166-170

148. D. Cline, W.F. Fry, Annu. Rev. Nucl. Part. Sci. 27, 209 (1977)

149. P.F. Ermolov, A.I. Mukhin, Sov. Phys. Usp. 21(3), 185 (1978)

150. S.I. Alekhin et al. (HERA and COMPASS Groups), Compilation of cross-sections $I V: \gamma, v, \Lambda, \Sigma, \Xi$, and $K_{L}^{0}$ induced reactions (CERN, High-Energy Reactions Analysis Group, Geneva, 1987)

151. M. Sakuda, Nucl. Phys. B (Proc. Suppl.) 112(1-3), 109 (2002)

152. V.V. Ammosov et al., Sov. J. Part. Nucl. 23(3), 283 (1992)

153. C. Baltay, Nucl. Phys. B (Proc. Suppl.) 36, 363 (1994)
154. M. Sorel, AIP Conf. Proc. 967, 17 (2007). arXiv:0710.3966 [hepex]

155. R. Gran, AIP Conf. Proc. 967, 141 (2007). arXiv:0711.3024 [hepex]

156. K. Abe et al. (T2K Collaboration), Phys. Rev. D 100(11), 112009 (2019). arXiv:1910.09439 [hep-ex]

157. K. Abe et al. (T2K Collaboration), Phys. Rev. D 101(11), 112001 (2020). arXiv:2002.09323 [hep-ex]

158. K. Abe et al. (T2K Collaboration), Phys. Rev. D 101(11), 112004 (2020). arXiv:2004.05434 [hep-ex]

159. K. Abe et al. (T2K Collaboration), Prog. Theor. Exp. Phys. 2019(9), 093C02 (2019). arXiv:1904.09611 [hep-ex]

160. K. Abe et al. (T2K Collaboration), Prog. Theor. Exp. Phys. 2021(4), 043 C01 (2021). arXiv:2004.13989 [hep-ex]

161. M.F. Carneiro et al. (MINERvA Collaboration), Phys. Rev. Lett. 124(12), 121801 (2020). arXiv:1912.09890 [hep-ex]

162. F. James, M. Roos, Comput. Phys. Commun. 10(6), 343 (1975). http://www.sciencedirect.com/science/article/pii/ 0010465575900399

163. F. James, MINUIT_function minimization and error analysis: Reference manual version 94.1 (1998). cERN Program Library Long Writeup D506. http://cdssls.cern.ch/record/2296388/files/ minuit.pdf

164. M. Rollier (Gargamelle Antineutrino Collaboration), Recent results from the Gargamelle $\bar{v}$ propane experiment at the CERNPS, in Proceedings of the Neutrino Physics at Accelerators, Topical Conference on Neutrino Physics, Oxford, England, UK, July 4-7, 1978, ed. by A.G. Michette, P.B. Renton (Science Research Council, Rutherford Laboratory, Chilton, 1978), pp. 68-74

165. A.A. Aguilar-Arevalo et al. (MiniBooNE Collaboration), Phys. Rev. Lett. 120(14), 141802 (2018). arXiv:1801.03848 [hep-ex]

166. I.D. Kakorin, K.S. Kuzmin, V.A. Naumov, Phys. Part. Nucl. Lett. 17(3), 265 (2020)

167. L.L. Salcedo, E. Oset, M.J. Vicente-Vacas, C. Garcia-Recio, Nucl. Phys. A 484(3-4), 557 (1988)

168. M. Alam et al., GENIE production release 2.10 .0 (2015). arXiv: 1512.06882 [hep-ph]

169. C. Andreopoulos et al., The GENIE neutrino Monte Carlo generator: physics and user manual, version 2.10 .0 (2015). arXiv:1510.05494 [hep-ph]

170. C. Andreopoulos et al. (GENIE Collaboration) (2019). Link to the instant release: https://genie-docdb.pp.rl.ac.uk/DocDB/0000/ 000002/003/man.pdf, see also https://genie-docdb.pp.rl.ac.uk/ DocDB/0000/000002/003/man.pdf

171. D. Ashery, I. Navon, G. Azuelos, H.K. Walter, H.J. Pfeiffer, F.W. Schleputz, Phys. Rev. C 23(5), 2173 (1981)

172. I. Navon, D. Ashery, J. Alster, G. Azuelos, B.M. Barnett, W. Gyles, R.R. Johnson, D.R. Gill, T.G. Masterson, Phys. Rev. C 28(6), 2548 (1983)

173. A.S. Carroll, I.H. Chiang, C.B. Dover, T.F. Kycia, K.K. Li, P.O. Mazur, D.N. Michael, P.M. Mockett, D.C. Rahm, R. Rubinstein, Phys. Rev. C 14(2), 635 (1976)

174. A.S. Clough et al., Nucl. Phys. B 76(1), 15 (1974)

175. W. Bauhoff, Atom. Data Nucl. Data Tables 35(3), 429 (1986)

176. L.M. Kerby, S.G. Mashnik, A.J. Sierk, Comparison of expanded preequilibrium CEM model with CEM 03.03 and experimental data (2014). arXiv:1401.4404 [nucl-th]

177. S.G. Mashnik, L.M. Kerby, Nucl. Instrum. Methods A 764, 59 (2014). arXiv:1404.7820 [nucl-th]

178. S.G. Mashnik, L.M. Kerby, K.K. Gudima, A.J. Sierk, J.S. Bull, M.R. James, Phys. Rev. C 95(3), 034613 (2017). arXiv: 1607.02506 [nucl-th]

179. S. Dytman, Acta Phys. Pol. B 40(9), 2445 (2009)

180. R.D. Mckeown, S.J. Sanders, J.P. Schiffer, H.E. Jackson, M. Paul, J.R. Specht, E.J. Stephenson, R.P. Redwine, R.E. Segel, Phys. Rev. C 24(1), 211 (1981) 
181. R.A. Arndt, I.I. Strakovsky, R.L. Workman, Int. J. Mod. Phys. A 18(3), 449 (2003)

182. R.A. Arndt, W.J. Briscoe, I.I. Strakovsky, R.L. Workman, Phys. Rev. C 74(4), 045205 (2006). arXiv:nucl-th/0605082

183. The SAID partial-wave analysis facility and full database are available at the website of George Washington University. http:// gwdac.phys.gwu.edu

184. J.M. Nieves, I. Ruiz Simo, M.J. Vicente Vacas, Phys. Rev. C 83(4), 045501 (2011). arXiv:1102.2777 [hep-ph]

185. A. Gil, J.M. Nieves, E. Oset, Nucl. Phys. A 627(4), 543 (1997). arXiv:nucl-th/9711009

186. A. Gil, J.M. Nieves, E. Oset, Nucl. Phys. A 627(4), 599 (1997). arXiv:nucl-th/9710070

187. J.M. Nieves, J.E. Amaro, M. Valverde, Phys. Rev. C 70(5), 055503 (2004). arXiv:nucl-th/0408005 [Erratum: ibid. 72(1), 019902(E) (2005)]

188. K.M. Graczyk, J.T. Sobczyk, Phys. Rev. D 77(5), 053001 (2008). arXiv:0707.3561 [hep-ph] [Erratum: ibid. 79(7), 079903(E) (2009)]

189. H. Gallagher, Nucl. Phys. B (Proc. Suppl.) 112, 188 (2002)

190. A.V. Baranov, N.A. Balashov, N.A. Kutovskiy, R.N. Semenov, Phys. Part. Nucl. Lett. 13(5), 672 (2016)

191. N.A. Balashov, M.V. Bashashin, R.I. Kuchumov, N.A. Kutovskiy, I.A. Sokolov, Mod. Inf. Tech. IT-Educ. 14(1), 61 (2018). http:// sitito.cs.msu.ru/index.php/SITITO/article/view/352/284

192. K. Gallmeister, U. Mosel, J. Weil, Phys. Rev. C 94(3), 035502 (2016). arXiv:1605.09391 [nucl-th]

193. J.E. Amaro, E. Ruiz Arriola, I. Ruiz Simo, Phys. Rev. C 92(5), 054607 (2015). arXiv:1505.05415 [nucl-th] [Erratum: ibid. 100(1), 019904 (2019)]

194. O. Buss, T. Gaitanos, K. Gallmeister, H. van Hees, M. Kaskulov, O. Lalakulich, A. Larionov, T. Leitner, J. Weil, U. Mosel, Phys. Rep. 512, 1 (2012). arXiv:1106.1344 [hep-ph]

195. J.E. Amaro, M.B. Barbaro, J.A. Caballero, T.W. Donnelly, A. Molinari, I. Sick, Phys. Rev. C 71(1), 015501 (2005). arXiv:nucl-th/0409078

196. G.D. Megias, J.E. Amaro, M.B. Barbaro, J.A. Caballero, T.W. Donnelly, Phys. Rev. D 94(1), 013012 (2016). arXiv:1603.08396 [nucl-th]

197. S. Dolan, G.D. Megias, S. Bolognesi, Phys. Rev. D 101(3), 033003 (2020). arXiv:1905.08556 [hep-ex]

198. J.E. Amaro, V.L. Martinez-Consentino, E. Ruiz Arriola, I. Ruiz Simo, Phys. Rev. C 98(2), 024627 (2018). arXiv: 1806.09512 [nucl-th]

199. T. Bonus, J.T. Sobczyk, M. Siemaszko, C. Juszczak, Phys. Rev. C 102(1), 015502 (2020). arXiv:2003.00088 [hep-ex]

200. I. Ruiz Simo, Private communication (2020)

201. The tabulated MiniBooNE data are taken from the BooNE Data Releases: https://www-boone.fnal.gov/for_physicists/ data_release/ccqe_nubar/ and https://www-boone.fnal.gov/for_ physicists/data_release/ccqe/

202. A.A. Aguilar-Arevalo et al. (MiniBooNE Collaboration), Phys. Rev. D 79(7), 072002 (2009). arXiv:0806.1449 [hep-ex]

203. A.A. Aguilar-Arevalo et al. (MiniBooNE Collaboration), Phys. Rev. D 84(7), 072005 (2011). arXiv:1102.1964 [hep-ex]

204. J.M. Grange, First measurement of the muon anti-neutrino charged current quasielastic double-differential cross section. Springer Theses (Springer International Publishing, Switzerland, 2015), Ph.D. Thesis, Florida University (2013). FERMILABTHESIS-2013-14
205. A.A. Aguilar-Arevalo et al. (MiniBooNE Collaboration), Phys. Rev. Lett. 103(8), 081801 (2009). arXiv:0904.3159 [hep-ex]

206. A.A. Aguilar-Arevalo et al. (MiniBooNE Collaboration), Phys. Rev. D 83(5), 052007 (2011). arXiv:1011.3572 [hep-ex]

207. C. Juszczak, Acta Phys. Pol. B 40(9), 2507 (2009). arXiv:0909.1492 [hep-ex]

208. T. Golan, C. Juszczak, J.T. Sobczyk, Phys. Rev. C 86(1), 015505 (2012). arXiv:1202.4197 [nucl-th]

209. K. Abe et al. (T2K Collaboration), Nucl. Instrum. Methods A 659(1), 106 (2011). arXiv:1106.1238 [physics.ins-det]

210. K. Abe et al. (T2K Collaboration), Phys. Rev. D 91(7), 072010 (2015). arXiv:1502.01550 [hep-ex]

211. S. Dolan, U. Mosel, K. Gallmeister, L. Pickering, S. Bolognesi, Phys. Rev. C 98(4), 045502 (2018). arXiv:1804.09488 [hep-ex]

212. Y. Hayato, Nucl. Phys. B (Proc. Suppl.) 112(1-3), 171 (2002)

213. Y. Hayato, Acta Phys. Pol. B 40(9), 2477 (2009)

214. R.W. Peelle, "Peelle's Perinent Puzzle" (1987). Informal memorandum dated October 13, 1987, Oak Ridge National Laboratory, Oak Ridge, Tennessee, USA

215. L. Aliaga et al. (MINERvA Collaboration), Phys. Rev. D 94(9), 092005 (2016). arXiv:1607.00704 [hep-ex] [Erratum: ibid. 95(3), 039903(E) (2017)]

216. M. Anfreville et al., Nucl. Instrum. Methods A 481(1-3), 339 (2002). arXiv:hep-ex/0104012

217. E.L. Lomon, Effect of revised $R_{n}$ measurements on extended Gari-Krümpelmann model fits to nucleon electromagnetic form factors (2006). arXiv:nucl-th/0609020

218. R. Petti, Measurements of quasi-elastic interactions in the NOMAD experiment, in A Talk at the INT Workshop INT-13-54W on Neutrino-Nucleus Interactions for Next Generation Neutrino Oscillation Experiments, Seattle, Washington, USA, December 3-13, 2013 (unpublished). http://www.int.washington.edu/talks/ WorkShops/int_13_54W/People/Petti_R/Petti.pdf

219. A. Bodek, Private communication (2008)

220. D. Allasia et al. (Amsterdam-Bergen-Bologna-Padua-PisaSaclay-Torino Collaboration), Nucl. Phys. B 239(2), 301 (1984)

221. L.A. Ahrens et al., Phys. Rev. D 34(1), 75 (1986)

222. T. Kitagaki et al., Phys. Rev. D 34(9), 2554 (1986)

223. F.J. Sciulli, AIP Conf. Proc. 22, 166 (1974)

224. H. Deden et al. (Gargamelle Neutrino Collaboration), Nucl. Phys. B 85(2), 269 (1975)

225. M. Sakuda, Study of neutrino-nucleus interactions for neutrino oscillation experiments, in Proceedings of the 4th Workshop on Neutrino Oscillations and Their Origin (NOON 2003), Kanazawa, Japan, February 10-14, 2003 (2003), pp. 253-260

226. K. Furuno et al., BNL 7-foot bubble chamber experiment: neutrino deuterium interactions, in Proceedings of the 2nd International Workshop on Neutrino-Nucleus Interactions in the Few GeV Region (NuInt 2002), Irvine, California, USA, December 12-15, 2002 (available from INSPIRE, 2003)

227. L.R. Sulak, eConf C7505091, 155 (1975) 